\title{
Wireless Sensing for Ground Engaging Tools
}

by

\author{
Mohamed Adel IBrahim \\ A thesis submitted to the \\ Department of Electrical and Computer Engineering \\ in conformity with the requirements for \\ the degree of Doctor of Philosophy
}

Queen's University

Kingston, Ontario, Canada

October 2021

Copyright @ () Mohamed Adel Ibrahim, 2021 


\section{Dedication}

I dedicate this work to God. I am deeply grateful for his bountiful blessings and encompassing mercy. 


\begin{abstract}
A long-standing industrial problem is tackled with an industrial internet of things (IIoT) application. This thesis presents the hardware design and development of a wireless sensor network for monitoring ground engaging tools of earth processing equipment. This work addresses several challenges in the hardware design. The challenges included establishing wireless communications from within a metallic enclosure, Investigating the effect of antenna tuning on the link budget, designing a protective package for the sensor node (SN) to withstand vibrations and mechanical shocks, and creating a low-power design that allowed for a minimum battery life of one year. Furthermore, this work puts forward the design of novel application-specific sensors including a low-power ferrous-selective proximity sensor and a low-cost capacitive wear-level sensor.

Hardware designs were evaluated with laboratory and field tests. The conducted tests demonstrated the SN's functionality and performance in harsh operating conditions. The journey from problem definition and to successful field testing of the complete wireless sensing solution is covered in this thesis.

In addition, a novel energy-efficient approach to wireless real-time sensing is presented. For an SN transmitting samples of a discrete time-series in real-time, its
\end{abstract}


lifetime depends primarily on its battery capacity. With most of the energy consumed in wireless transmission, this thesis introduces an energy-efficient scheme that significantly reduces the number of transmitted samples, while maintaining a low mean absolute error between the original and the recovered signal. The concept of instantaneous entropy is introduced and a computationally-efficient iterative formula for computing Shannon's entropy is derived. The SN evaluates the information content in each sample and decide whether to transmit or omit the sample. At the sink, incremental machine learning is used to recover the omitted samples in real-time. This approach shows an average of $60 \%$ reduction in energy consumption by the SN with less than $2 \%$ mean absolute error in the recovered signal. The simulation employed stationary, non-stationary, chaotic, and random time-series for performance evaluation and benchmarking. 


\section{Disclaimer}

This work was made possible through the software developed by Galal Hassan. His work on the shovel project covered firmware development for the sensor node, software development for the sink, and setting up the back-end cloud server including the

dashboard. Furthermore, Galal has contributed significantly to the success of the shovel project by developing an energy-efficient MAC protocol [1] which allowed for satisfying the life-time requirement of the sensor nodes. 


\section{Co-Authorship}

- M. A. Ibrahim, G. Hassan, H. S. Hassanein, and K. Obaia, "A wireless sensor platform for industrial non-hermetic metallic enclosures," in 13th International Wireless Communications and Mobile Computing Conference (IWCMC), Jun. 2017, pp. 165-170, doi: 10.1109/IWCMC.2017.7986280.

- G. Hassan, M. ElMaradny, M. A. Ibrahim, A. M. Rashwan, and H. S. Hassanein, "Energy Efficiency Analysis of Centralized-Synchronous LoRa-based MAC Protocols," in 14th International Wireless Communications Mobile Computing Conference (IWCMC), Jun. 2018, pp. 999-1004, doi: 10.1109/IWCMC.2018.8450523.

- M. A. Ibrahim, G. Hassan, K. Monea, H. S. Hassanein, and K. Obaia, "A Ferrous-Selective Proximity Sensor for Industrial Internet of Things," in IEEE International Conference on Communications (ICC), Jun. 2020, pp. 1-6, doi: 10.1109/ICC40277.2020.9148958.

- M. A. Ibrahim, G. Hassan, H. S. Hassanein, and K. Obaia, "Wireless Sensing for Ground Engaging Tools," in IEEE Global Communications Conference (GLOBECOM), Dec. 2020, pp. 1-6, doi: 10.1109/GLOBECOM42002.2020.9322336. 
- M. A. Ibrahim, H. S. Hassanein, "Entropic Sensing for Energy Efficiency," in 18th IEEE International Conference on Mobile Ad Hoc and Smart Systems (MASS), Oct. 4-7, 2021.

- M. A. Ibrahim, G. Hassan, H. S. Hassanein, and K. Obaia, "Wireless Sensing System for Monitoring Ground Engaging Tools," in IEEE Internet of Things Journal, Submitted May 9, 2021. 


\section{Acknowledgements}

I am sincerely grateful for working under the supervision of Dr. Hossam Hassanein. In addition to his outstanding academic support throughout a long journey, he successfully cultivated innovation, fostered intellectual and professional development through my $\mathrm{PhD}$ program.

Galal Hassan is my talented lab mate, colleague, and dear friend who supported this work with his strong and versatile software development skills, his multidisciplinary experience, and his profound commitment towards the end goal. I am forever in dept to his significant contributions.

Basia Palmer is my kind and highly skilled colleague and mentor who coordinated the research activities on the shovel project, gracefully managed the logistics, and proof-read my written work while training and educating me in the process. Working with Basia is a privilege that I will always cherish.

Abdollah Safar is a bright engineer who worked for our industrial partner. He was the ultimate practicality gateway. His deep understanding of the mining environment and his ability to identify unforeseen risks introduced major contributions to the system implementation and its success in the field.

Dr. Khaled Obaia is the industrial lead on the shovel project. He showed a deep level of commitment to our work and guided our efforts along the way. I am thankful 
for his guidance and insights during this journey.

Many other talented and kindhearted people contributed to this work. I am deeply grateful for their time, and effort. Dr. Abdallah Almaitah, Dr. A. Rashwan, Dr. Hesham Eldesouky, Dr. Abdalla Abdelrahman, Mohamed El-Maradny, Katerina Monea, Amr Magdy, Jean-François Raffier. I am thankful for their contributions and grateful for their help and support.

On the personal side, I acknowledge my wonderful family. My loving mother Nadia who always cared for me and supported my academic endeavours in every way possible. My father Adel is my mentor, life coach, and best companion.

My wife Gehad is my beating heart, my guiding light, and my haven. I am forever grateful for her constant support and her unconditional love. My daughter Janna is the perfect example of a pure soul. May her pursuit of knowledge and wisdom be rewarded. My son Shadi is the source of joy in our family. I wish him a fulfilling rewarded life. My son Ali is the source of kindness in our family. I wish him a fulfilling rewarded life.

My brother Islam is my hero, leader, and my sense of safety and security. God bless him, his loving wife Melissa, and their wonderful kids: Malik, Aya, and Mazin.

My friends are my refuge from the bitterness of life. I am thankful for their company and support. 


\section{Contents}

Dedication $\quad$ i

Abstract

Disclaimer $\quad$ iv

Co-Authorship $\quad$ v

Acknowledgements vii

Acronyms $\quad$ xx

Chapter 1: Introduction 1

1.1 Motivation and Objectives .................. 4

1.2 Academic Contributions . . . . . . . . . . . . . . . 7

1.3 Industrial Contributions . . . . . . . . . . . . . . . . . 7

1.4 Thesis Organization ................... . . 8

$\begin{array}{lll}\text { Chapter 2: } & \text { Background } & 9\end{array}$

2.1 Hardware Platforms . . . . . . . . . . . . . . . . . 9 9

2.1.1 Hardware Architecture . . . . . . . . . . . . . . . . . 13

2.2 Monitoring Ground Engaging Tools . . . . . . . . . . . . . . . . . . . 17

ix 
2.2.1 Image-Based Solutions . . . . . . . . . . . . . . . . . . 17

2.2.2 Wireless Sensing Solutions . . . . . . . . . . . . . . 18

2.3 Summary . . . . . . . . . . . . . . . . . . . . . 20

Chapter 3: $\quad$ System Design 21

3.1 Sink . . . . . . . . . . . . . . . . . . . 30

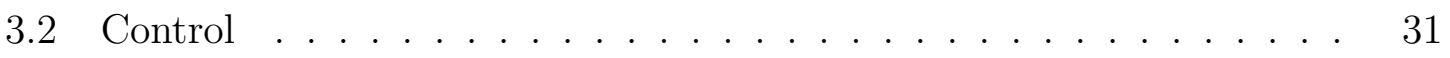

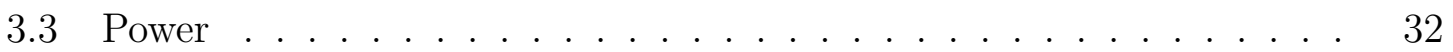

3.4 Communication . . . . . . . . . . . . . . . . . 34

3.4.1 Approach .................... 36

3.4 .2 Experiment . . . . . . . . . . . . . . . 38

3.4.3 Antenna Matching . . . . . . . . . . . . . . . . . . . . 44

3.4.4 Cavity Mock-Up . . . . . . . . . . . . . . . . . . . 46

3.4.5 MAC Protocol ................... 50

3.5 Sensing . . . . . . . . . . . . . . . . . 51

3.5.1 Ferrous-Selective Proximity Sensing . . . . . . . . . . . . . 54

3.5.2 Capacitive Proximity Sensor (Bucket Side) . . . . . . . . . . . 70

3.5.3 Capacitive Proximity Sensor (Adapter Side) . . . . . . . . . 71

3.5.4 Inductive Proximity Sensor . . . . . . . . . . . . . . . . 71

3.5.5 Wear-Level Sensor . . . . . . . . . . . . . . . . . 72

3.5.6 Light Sensor . . . . . . . . . . . . . . . . . . . 73

3.5.7 Vibration Sensor . . . . . . . . . . . . . . . 76

3.5.8 Temperature Sensor . . . . . . . . . . . . . . . . . . . 77

3.5.9 Battery Voltage Sensor . . . . . . . . . . . . . . . . 77

3.6 Summary . . . . . . . . . . . . . . . . . . 77 
$\begin{array}{lll}\text { Chapter 4: } & \text { Implementation and Testing } & 78\end{array}$

4.1 Package Design . . . . . . . . . . . . . . . . 80

4.1 .1 Impact Test . . . . . . . . . . . . . . . . . 83

4.1.2 Packaging Process . . . . . . . . . . . . . . . . . 84

4.2 Communication . . . . . . . . . . . . . . . . . . . . 90

4.2 .1 Range . . . . . . . . . . . . . . . . . . . 90

4.2.2 Antenna Matching . . . . . . . . . . . . . . . 92

4.2.3 Buried Sensor Node Communication . . . . . . . . . . . . . 93

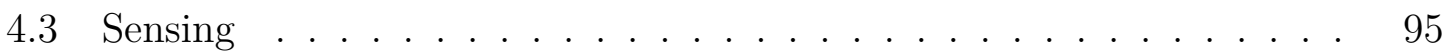

4.3.1 Proximity Sensors . . . . . . . . . . . . . . . 95

4.3.2 Wear-Level Sensor . . . . . . . . . . . . . . . . . . 99

4.4 Power ................................ 102

4.5 Summary . . . . . . . . . . . . . . . . 103

$\begin{array}{ll}\text { Chapter 5: } & \text { Entropic Sensing }\end{array}$

5.1 Introduction . . . . . . . . . . . . . . . . . 104

5.1.1 Existing Approaches . . . . . . . . . . . . . . . 106

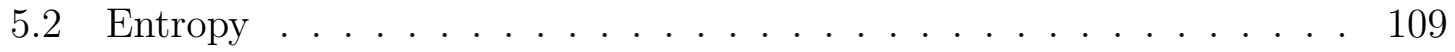

5.3 Formulation . . . . . . . . . . . . . . . . 113

5.3.1 Assumptions ....................... 114

5.3.2 Generalizations ................... 115

5.3.3 Benchmarking Time-Series . . . . . . . . . . . . . 116

5.4 Entropic Filter . . . . . . . . . . . . . . . . . . . . . 118

5.4.1 Computing Entropy . . . . . . . . . . . . . . 123

5.4 .2 Probability Shaping Function . . . . . . . . . . . . . 128 
5.5 Recovery. . . . . . . . . . . . . . . . . . 132

5.6 Evaluation ............................ 133

5.6.1 Memory Requirements . . . . . . . . . . . . . . 137

5.6.2 Energy Savings . . . . . . . . . . . . . . . . . . . 138

5.7 Summary . . . . . . . . . . . . . . . . . . . 139

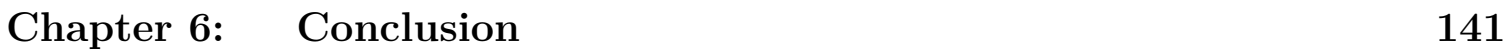

6.1 Future Directions . . . . . . . . . . . . . . . . . . 143

6.1.1 Communication .................. 143

6.1.2 Ferrous-Selective Magnetic Force Proximity Sensor . . . . . . 144

6.1.3 Wear-Level Sensor . . . . . . . . . . . . . . . . . . . 144

6.1.4 Capacitive Proximity Sensor . . . . . . . . . . . . . . . . 144

6.1.5 Entropic Sensing . . . . . . . . . . . . . . . 145

6.2 Development Recommendations . . . . . . . . . . . . . . . . 146

$\begin{array}{lr}\text { Bibliography } & 149\end{array}$

$\begin{array}{ll}\text { Appendix A: Circuit Diagrams } & 170\end{array}$

$\begin{array}{ll}\text { Appendix B: Entropic Sensing Plots } & 176\end{array}$

B.1 Entropic Filtering . . . . . . . . . . . . . . 176

B.2 Computing Entropy . . . . . . . . . . . . . . . . . . 188

B.3 Probability Shaping . . . . . . . . . . . . . . . . 194

B.4 Evaluation . . . . . . . . . . . . . . . . . . 200 


\section{List of Tables}

3.1 Breakdown of adapter detachment events. . . . . . . . . . . . . 24

3.2 Rated power consumption of devices onboard PCB-full . . . . . . . . 33

3.3 A summary of the utilized radio parameters. . . . . . . . . . . . . . 44

3.4 Specifications of components used in the experiment. . . . . . . . 65

4.1 Properties of the encapsulating silicone rubber (MoldMax: 14NV). . . 82 


\section{List of Figures}

1.1 Various types of Ground Engaging Tools . . . . . . . . . . . . . 6

2.1 Sprouts hardware architecture. . . . . . . . . . . . . . . 12

2.2 The block diagram of a typical sensor node. . . . . . . . . . . . . . . 14

3.1 The first stage in an open-pit oil sands mining process. . . . . . . . . 22

3.2 The adapter/tooth assembly of the designated electric rope shovel. . 24

3.3 Network diagram. . . . . . . . . . . . . . . . . . . . 25

3.4 System block diagram. . . . . . . . . . . . . . . . . 26

3.5 System overview. . . . . . . . . . . . . . . . . . . . 27

3.6 A detailed block diagram of our sensor node. . . . . . . . . . . . 28

3.7 A summary of the sensor node design requirements tree. . . . . . . . 29

3.8 Block diagram of the sink hardware setup. . . . . . . . . . . . . . 31

3.9 Classification of industrial enclosures. . . . . . . . . . . . . . 35

3.10 CAD drawing of the NHM enclosure. . . . . . . . . . . . . . . 40

3.11 The NHM enclosure and the sensor platform inside its cavity. . . . . 40

3.12 The orientation of the enclosure. . . . . . . . . . . . . . 41

3.13 The experimental setup. . . . . . . . . . . . . . . . . 41

3.14 Received signal strength at 30 m range. . . . . . . . . . . . . . 43

3.15 The shovel adapter and the gap exploited for wireless communication. 43 
3.16 The schematic diagram of the LoRa ${ }^{\mathrm{TM}}$ transceiver . . . . . . . . . . 45

3.17 The 3D printed cavity mock-up. . . . . . . . . . . . . 47

3.18 Experimental setup for tuning the antenna. . . . . . . . . . . . . . . 48

3.19 The Smith chart used to calculate the matching network components. $\quad 49$

3.20 The simulated performance of the matching network. . . . . . . . . 50

3.21 Classification of proximity sensors. . . . . . . . . . . . . . 52

3.22 The MAGNASPHERE ferrous-selective PSs. . . . . . . . . . . . . . . 54

3.23 Design of the Ferrous-Selective Magnetic-Force Proximity Sensor . . . 57

3.24 Empirical model of the magnetic attraction force. . . . . . . . . . . . 60

3.25 The experimental setup on the UTM . . . . . . . . . . . . 64

3.26 Target's proximity vs. attraction force. . . . . . . . . . . . 67

3.27 Target's proximity vs. attraction force. . . . . . . . . . . 68

3.28 Target's proximity vs. attraction force. . . . . . . . . . . . 68

3.29 Output comparison of the three magnets. . . . . . . . . . . . . . 69

3.30 The Polycarbonate support. . . . . . . . . . . . . . . . . 71

3.31 The wear-level sensor. . . . . . . . . . . . . . . . . . . 73

3.32 The circuit diagram of the ambient light sensor. . . . . . . . . . . . . 74

3.33 The dynamic range of the light sensor. . . . . . . . . . . . . . 76

4.1 The dashboard top. . . . . . . . . . . . . . . . . . . . 79

4.2 The dashboard bottom. . . . . . . . . . . . . . . . . 79

4.3 Probability Mass Function of daily extreme temperatures. . . . . . . 81

4.4 Dimensions $(\mathrm{m})$ of the electric rope shovel [2]. . . . . . . . . . . . 81

4.5 An illustration of the package. . . . . . . . . . . . . . . 82

4.6 Full PCB closeup. . . . . . . . . . . . . . . . . . 85 
4.7 Full PCB after coating with liquid electrical tape. . . . . . . . . . 85

4.8 Reduced PCB after coating with liquid electrical tape. . . . . . . . . 86

4.9 Assembled batch of SNs before packaging. . . . . . . . . . . . . . 86

4.10 The 3D printed rubber mould after post processing. . . . . . . . . . 87

4.11 Rubber pouring setup. . . . . . . . . . . . . . . . . 88

4.12 Full PCB after coating with liquid electrical tape. . . . . . . . . . 88

4.13 Partially potted SNs after curing the rubber. . . . . . . . . . . . 88

4.14 A batch of SNs before being covered with the fibreglass sleeve. . . . . 89

4.15 An SN being covered with the fibreglass sleeve. . . . . . . . . . . . 89

4.16 The opening in the fibreglass weave. . . . . . . . . . . . . . 90

4.17 Fully packaged SNs. . . . . . . . . . . . . . . . . . . . . . 90

4.18 A violin plot of the communication range. . . . . . . . . . . . . 91

4.19 Tuned versus untuned nodes . . . . . . . . . . . . . . . 93

4.20 First communication test, buried SN. . . . . . . . . . . . . . . . 94

4.21 Second communication test. buried SN. . . . . . . . . . . . . . . . . . 94

4.22 Sensor readings from an attaching/detaching test in the workshop. . . 96

4.23 The sensor node inside the adapter. . . . . . . . . . . . . . . . . 97

4.24 A photo from the sink node's location on a rail next to the cabin. . . 97

4.25 Sensor data collected from an operational shovel during the field test. $\quad 98$

4.26 Operator dashboard on the cabin tablet. . . . . . . . . . . . . . 100

4.27 Wear level sensor capacitance vs its length. . . . . . . . . . . . . . . . 102

4.28 Current consumption profile for a single duty cycle of the SN. . . . . 103

5.1 The signal path in a typical WSN. . . . . . . . . . . . . . 106

5.2 The signal path in the introduced entropic sensing system. . . . . . . 113 
5.3 The flow chart for entropic filtering. . . . . . . . . . . . . 120

5.4 Entropic filtering steps for S6. . . . . . . . . . . . . . . . . 122

5.5 The discrete time-series and its IE. . . . . . . . . . . . . . . . 128

5.6 The four probability shaping functions used in this work. . . . . . . 130

5.7 Histogram of $\check{r}$ for $X$ and $d X$ of $S 5 . \ldots \ldots \ldots \ldots \ldots$

5.8 Flow chart of the predictor's operation inside the sink. . . . . . . . 132

$5.9 \overline{|e|}$ and $S R R$ among all the probability shaping functions for $S 5 \ldots 135$

5.10 Top: $X$ and $\hat{X}$. Bottom: Instantaneous absolute error $|e| . \ldots \ldots$

5.11 Average Euclidean error $(\overline{|e|})$ of $p s f 1 \ldots \ldots \ldots \ldots \ldots$

A.1 Schematic diagram of the full featured PCB (Sheet 1). . . . . . . 171

A.2 Schematic diagram of the full featured PCB (Sheet 2) . . . . . . 172

A.3 Schematic diagram of the full featured PCB (Sheet 3). . . . . . . 173

A.4 Schematic diagram of the reduced PCB (Sheet 1) . . . . . . . . 173

A.5 Schematic diagram of the reduced PCB (Sheet 2). . . . . . . . 174

A.6 Schematic diagram of the reduced PCB (Sheet 3). . . . . . . 175

B.1 Entropic filtering steps for $S 1 \ldots \ldots \ldots \ldots \ldots \ldots \ldots$

B.2 Entropic filtering steps for $S 2 \ldots \ldots \ldots \ldots \ldots \ldots \ldots$

B.3 Entropic filtering steps for $S 3 \ldots \ldots \ldots \ldots \ldots \ldots \ldots$

B.4 Entropic filtering steps for $S 4 \ldots \ldots \ldots \ldots \ldots \ldots \ldots$

B.5 Entropic filtering steps for $S 5 \ldots \ldots \ldots \ldots \ldots \ldots \ldots \ldots$

B.6 Entropic filtering steps for $S 6 \ldots \ldots \ldots \ldots \ldots \ldots$

B.7 Entropic filtering steps for $S 7 \ldots \ldots \ldots \ldots \ldots \ldots \ldots$

B.8 Entropic filtering steps for $S 8 \ldots \ldots \ldots \ldots \ldots \ldots \ldots \ldots$

B.9 Entropic filtering steps for $S 9 \ldots \ldots \ldots \ldots \ldots \ldots \ldots$ 
B.10 Entropic filtering steps for $S 10 \ldots \ldots \ldots \ldots \ldots \ldots$

B.11 Entropic filtering steps for $S 11 \ldots \ldots \ldots \ldots \ldots \ldots \ldots$

B.12 $S 1$ and its instantaneous Entropy . . . . . . . . . . . 189

B.13 S2 and its instantaneous Entropy . . . . . . . . . . . . . 189

B.14 S3 and its instantaneous Entropy . . . . . . . . . . . 190

B.15 S4 and its instantaneous Entropy . . . . . . . . . . . 190

B.16 S5 and its instantaneous Entropy . . . . . . . . . . . . 191

B.17 S6 and its instantaneous Entropy . . . . . . . . . . . . 191

B.18 S7 and its instantaneous Entropy . . . . . . . . . . . . 192

B.19 S8 and its instantaneous Entropy . . . . . . . . . . . . . 192

B.20 S9 and its instantaneous Entropy . . . . . . . . . . . 193

B.21 S10 and its instantaneous Entropy $\ldots \ldots \ldots \ldots \ldots$

B.22 S11 and its instantaneous Entropy _. . . . . . . . . . . 194

B.23 PDF of $\check{r}_{X}$ and $\check{r}_{d X}$ for $S 1 \ldots \ldots \ldots \ldots \ldots \ldots \ldots$

B.24 PDF of $\check{r}_{X}$ and $\check{r}_{d X}$ for $S 2 \ldots \ldots \ldots \ldots \ldots \ldots \ldots$

B.25 PDF of $\check{r}_{X}$ and $\check{r}_{d X}$ for $S 3 \ldots \ldots \ldots \ldots \ldots \ldots \ldots \ldots$

B.26 PDF of $\check{r}_{X}$ and $\check{r}_{d X}$ for $S 4 \ldots \ldots \ldots \ldots \ldots \ldots \ldots$

B.27 PDF of $\check{r}_{X}$ and $\check{r}_{d X}$ for $S 5 \ldots \ldots \ldots \ldots \ldots \ldots \ldots \ldots \ldots \ldots$

B.28 PDF of $\check{r}_{X}$ and $\check{r}_{d X}$ for $S 6 \ldots \ldots \ldots \ldots \ldots \ldots \ldots \ldots \ldots \ldots \ldots$

B.29 PDF of $\check{r}_{X}$ and $\check{r}_{d X}$ for $S 7 \ldots \ldots \ldots \ldots \ldots \ldots$

B.30 PDF of $\check{r}_{X}$ and $\check{r}_{d X}$ for $S 8 \ldots \ldots \ldots \ldots \ldots \ldots$

B.31 PDF of $\check{r}_{X}$ and $\check{r}_{d X}$ for $S 9 \ldots \ldots \ldots \ldots \ldots \ldots \ldots \ldots$

B.32 PDF of $\check{r}_{X}$ and $\check{r}_{d X}$ for $S 10 \ldots \ldots \ldots \ldots \ldots \ldots$

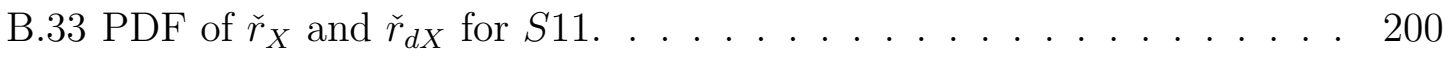


B.34 $\overline{|e|}$ and $S R R$ among all the PSFs for $S 1 \ldots \ldots \ldots \ldots \ldots$

B.35 $\overline{|e|}$ and $S R R$ among all the PSFs for $S 2 \ldots \ldots \ldots \ldots . \ldots . \ldots . \ldots$

B.36 $\overline{|e|}$ and $S R R$ among all the PSFs for $S 3 \ldots \ldots \ldots \ldots . \ldots . \ldots 202$

B.37 $\overline{|e|}$ and $S R R$ among all the PSFs for $S 4 \ldots \ldots \ldots \ldots . \ldots . \ldots 202$

B.38 $\overline{|e|}$ and $S R R$ among all the PSFs for $S 5 \ldots \ldots \ldots \ldots \ldots$

B.39 $\overline{|e|}$ and $S R R$ among all the PSFs for $S 6 \ldots \ldots \ldots \ldots$

B.40 $\overline{|e|}$ and $S R R$ among all the PSFs for $S 7 \ldots \ldots \ldots \ldots . \ldots . \ldots$

B.41 $\overline{|e|}$ and $S R R$ among all the PSFs for $S 8 \ldots \ldots \ldots \ldots \ldots$

B.42 $\overline{|e|}$ and $S R R$ among all the PSFs for $S 9 \ldots \ldots \ldots \ldots \ldots$

B.43 $\overline{|e|}$ and $S R R$ among all the PSFs for $S 10 \ldots \ldots \ldots \ldots$

B.44 $\overline{|e|}$ and $S R R$ among all the PSFs for $S 11 \ldots \ldots \ldots \ldots$

B.45 Top: $X$ and $\hat{X}$. Bottom: Instantaneous absolute error $|e| . \ldots .207$

B.46 Top: $X$ and $\hat{X}$. Bottom: Instantaneous absolute error $|e| . \ldots \ldots 207$

B.47 Top: $X$ and $\hat{X}$. Bottom: Instantaneous absolute error $|e| . \ldots .208$

B.48 Top: $X$ and $\hat{X}$. Bottom: Instantaneous absolute error $|e| . \ldots .208$

B.49 Top: $X$ and $\hat{X}$. Bottom: Instantaneous absolute error $|e| . \quad \ldots \ldots .209$

B.50 Top: $X$ and $\hat{X}$. Bottom: Instantaneous absolute error $|e| . \ldots . .209$

B.51 Top: $X$ and $\hat{X}$. Bottom: Instantaneous absolute error $|e| . \ldots . .210$

B.52 Top: $X$ and $\hat{X}$. Bottom: Instantaneous absolute error $|e| . \ldots . .210$

B.53 Top: $X$ and $\hat{X}$. Bottom: Instantaneous absolute error $|e| . \ldots \ldots 211$

B.54 Top: $X$ and $\hat{X}$. Bottom: Instantaneous absolute error $|e| . \ldots . .211$

B.55 Top: $X$ and $\hat{X}$. Bottom: Instantaneous absolute error $|e| . \quad \ldots \ldots .212$ 


\section{Acronyms}

ADC Analog Digital Converter

BLE Bluetooth Low Energy

BOM Bill of materials

CAD Computer Aided Design

CDC Capacitance Digital Converter

CSS Chirp Spread Spectrum

DFM Design for Manufacturing

EF Entropic Filter

EM Electromagnetic

EMI Electromagnetic Interference

ES Entropic Sensing

FSR Force Sensing Resistor 
GET Ground Engaging Tools

GPIO General Purpose Input/Output

HM Hermetic Metallic

HP Hardware Platform

I2C Inter-Integrated Circuit

IDC Inductance Digital Converter

IE Instantaneous Entropy

IIoT Industrial Internet of Things

IoT Internet of Things

IPC Institute of Printed Circuits

ISM Industrial Scientific Medical

LDR Light Detecting Resistor

LoRa $^{\mathrm{TM}}$ Long Range

LPWAN Low-Power Wide Area Networks

LSTM Long Short Term Memory

MAC Medium Access Control

MoT MAC On Time 
NHM Non-hermetic Metallic

PC Polycarbonate

PCB Printed Circuit Board

PnP Plug and Play

PS Proximity Sensor

PSF Probability Shaping Function

PWM Pulse Width Modulation

RF Radio Frequency

RFID Radio Frequency Identification

RSS Received Signal Strength

SN Sensor Node

SoC System on Chip

SPI Serial Peripheral Interface

UART Universal Asynchronous Receiver Transmitter

UTM Universal Testing Machine

WSN Wireless Sensor Network 


\section{Chapter 1}

\section{Introduction}

The Internet of Things (IoT) is "a dynamic global network infrastructure with selfconfiguring capabilities based on standard and interoperable communication protocols where physical and virtual 'Things' have identities, physical attributes, and virtual personalities and use intelligent interfaces, and are seamlessly integrated into the information network" [3]. The IoT has various applications in almost every economic sector, its market size is growing globally and expected to reach 1.6 trillion U.S. dollars by 2025 [4]. Many underlying technologies contributed to the ubiquity of the IoT [5]. Examples include, Radio Frequency Identification (RFID), cloud computing, wireless networks, and by extension, microelectronics.

IoT with an industrial scope are called Industrial Internet of Things (IIoT). The solutions offered by the IIoT bring significant improvements to the operation of industrial systems. For example, in production lines, the deployment of wireless sensing devices can enable predictive maintenance, highlight cost reduction opportunities, and inform quality assurance processes. Another example, in mining operations, the vertical integration of heterogeneous technologies can streamline processes, expose unidentified risks, and improve efficiency. 
A major enabler for IIoT applications is the Wireless Sensor Network (WSN) [6]. A WSN is a network of wireless sensing devices called Sensor Nodes (SNs). Despite the challenges of WSNs [7-9], they are finding their way into lots of applications, simply because of their low cost, improved scalability and versatility compared to their wired alternative.

In general, an SN is a hardware electronic device with limited energy and computing resources. The $\mathrm{SN}$ is application-specific and is a crucial piece of hardware responsible for sensing and communicating all the required sensory data. Also, the SN must withstand environmental and operating conditions throughout its lifetime. The success or failure of a certain wireless sensing application hinges largely on the performance of SNs.

In some domains, the spread of WSNs is challenged by the harsh conditions in which the SN is required to operate. Rugged electronics usually come at a hefty price tag which is contrary to what WSNs are aiming to offer. Lately, there has been interest in developing low-cost hardware that can operate in harsh environments [10]. This allows for digitizing industrial processes that have been previously considered too harsh for embedded electronics.

Every IIoT application has its own requirements, which springs from a unique set of operating conditions, performance goals, and design constraints. Since application requirements are the main driver of hardware design decisions, therefore each application would need its own custom designed SN. This is in line with the conclusion of the authors in [11] after a thorough review of the hardware specifications for over 40 different SNs. 
As industrial systems become smarter and more digitized, embedded electronics are expected to spread into new domains [12-14]. Thus, communicating with electronics embedded in various industrial assets becomes difficult without dedicated and more capable wireless technologies. Popular wireless technologies used in WSNs include RFID, Zigbee, Bluetooth Low Energy (BLE), and Long Range (LoRa ${ }^{\mathrm{TM}}$ ).

RFID is a wireless technology developed for remotely and uniquely identifying tagged objects, people, or other living creatures. RFID is applied in many domains including supply chain [15], access control [16], live stock management [17], manufacturing [18], shipping [19], postal services [20], and healthcare [21]. A typical RFID system consists of a reader and a number of attachable tags. When a tagged object is in close vicinity to the reader, the tag receives a wireless interrogation signal which instructs the tag to reply with its unique identification code. The tag can be active or passive. Active tags offer more functionality and extended interrogation ranges, but are more complex and relatively expensive. Passive tags usually harvest the reader's Radio Frequency (RF) energy to power up a low-cost printed circuit long enough just to reply back with its identification code.

Zigbee is a wireless communication standard [22] produced by a group of companies and industry organizations called the Zigbee alliance, which was recently rebranded to become the Connectivity Standards Alliance (CSA) [23]. In a layered networking model, Zigbee inherits the physical and the Medium Access Control (MAC) layers from the IEEE 802.15.4 standard, then it specifies its own network layer and provides a framework for the application layer. The Zigbee protocol has been adopted by many wireless products and is used in WSNs [24] [25], and IoT applications [26].

Bluetooth is also a wireless communication standard produced by a group of 
industry leaders back in 1998 [27] called the Bluetooth special interest group [28]. Ever since, the standard has evolved significantly over the years and was extensively adopted by many products. The BLE standard offers an energy-efficient variant of the Bluetooth standard. BLE is more tailored for wireless sensors and energy constrained devices in general. It is used in many WSNs, and IoT applications.

$\mathrm{LoRa}^{\mathrm{TM}}$ is another telecommunication standard aimed at Low-Power Wide Area Networks (LPWAN). It started in 2015 and grew quickly to be adopted by many products in the IoT domain. The standard is produced and maintained by the LoRa

alliance [29]. Unlike Zigbee, LoRa ${ }^{\mathrm{TM}}$ has its own physical layer specification which was designed with energy-efficiency in mind. On top of the physical layer, LoRa ${ }^{\mathrm{TM}}$ offers the LoRaWAN networking protocol to allow interoperability between different devices. The adoption level of LoRa ${ }^{\mathrm{TM}}$ is steadily increasing.

Currently available telecommunication technologies offer a solid foundation for novel and creative applications. This thesis builds on the current technology to develop an industrial application and uses theoretical concepts to help tackle the energy efficiency challenge of wireless sensing devices.

\subsection{Motivation and Objectives}

This work presents the design and development of a WSN in a mining application that can very well benefit applications in other sectors such as construction, and agriculture. Furthermore, inspired by the challenge of the SN's limited energy budget, a novel sensing techniques is developed using an information-theoretic approach to reduce the energy consumption of SNs in real-time monitoring applications. 
In mining, construction, and agriculture, large fleets of earth processing equipment require a consumable part called a ground engaging tool such as shovel teeth, side cutters, dozer blades, rippers, chisels, hammers, as shown in Figure 1.1. Such equipment and machines regularly consume significant number of Ground Engaging Tools (GET).

During operation, GET are exposed to significant levels of fatigue [31], and abrasion [32]. Consequently, near the end of their service life, they become prone to structural failures, which often result in a sudden detachment from the equipment. A ground engaging tool is a heavy chunk of steel; if it ends up in the wrong place, it can cause significant equipment damage, and sometimes human injury [33]. Thus, they require continuous monitoring for regular and timely replacement.

This thesis presents the hardware design and development of a WSN which was deployed inside the teeth of an operational electric rope shovel in an oil-sand mine in northern Alberta, Canada. Considering the harsh environmental and operating conditions of the SN, its success presents valuable lessons in the design of wireless hardware platforms for harsh conditions such as vibrations, mechanical shocks, dirt, humidity, and extreme temperatures. Also, the challenge of establishing wireless communication with an SN installed inside a metallic enclosure is addressed, which puts forward a successful case for embedding wireless sensors inside metallic parts. 


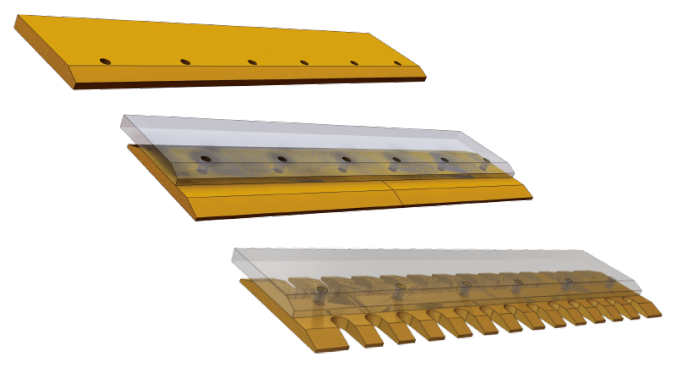

(a)

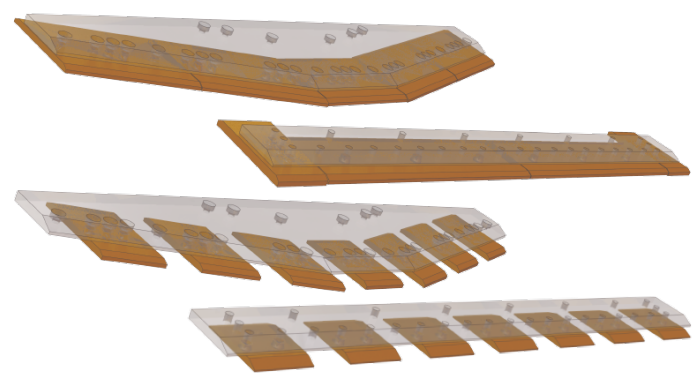

(c)

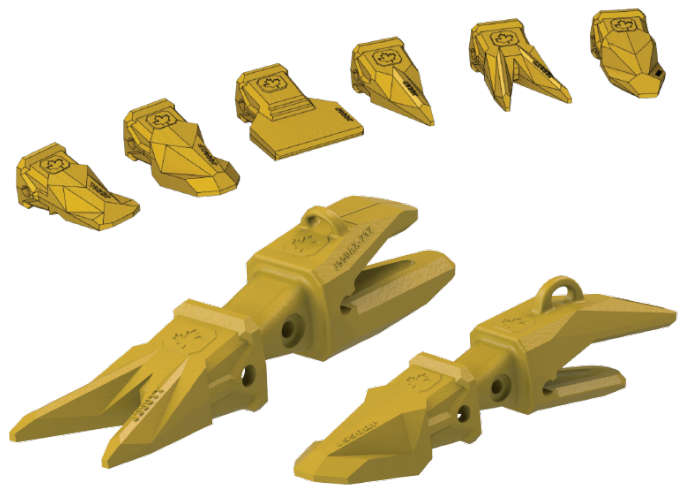

(b)

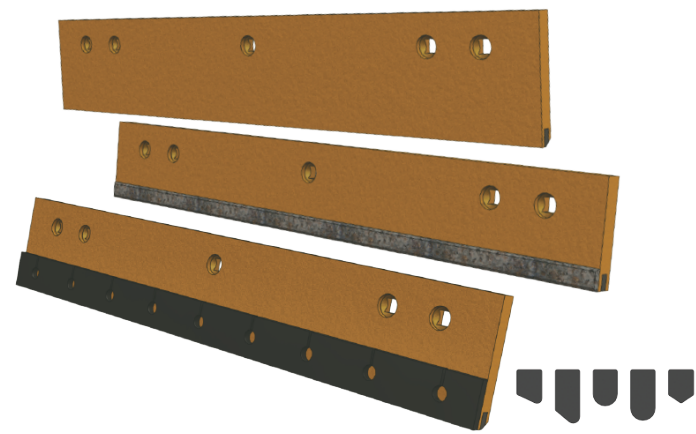

(d)

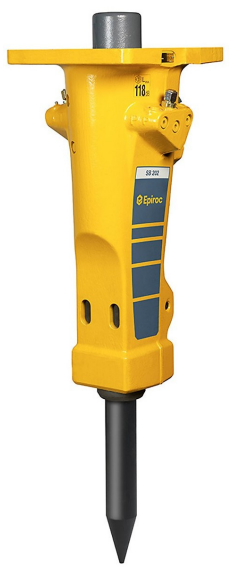

(e)

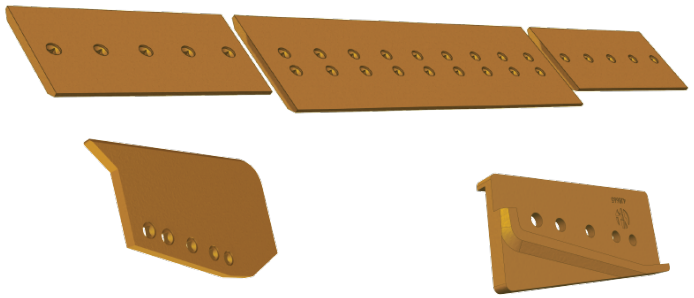

(f)

Figure 1.1: Various types of Ground Engaging Tools [30] used in mining/construction/agriculture machinery. (a) Excavator blades, (b) Shovel teeth and adapters, (c) loader-blades, (d) snow-plow-blades, (e) hydraulic-hammer, (f) scraper-blades. 


\subsection{Academic Contributions}

1. Validation of wireless communication from within a non-hermetic metallic container without a custom antenna [34].

2. Design and implementation of a low-power ferrous-selective proximity sensor more suited for wireless sensors [35].

3. Design and implementation of a low-cost, low-power, capacitive thickness probe which allows for measuring wear on a metal part with minimal change to its mechanical structure (Chapter 3).

4. Derivation of a maximum dynamic range formula for voltage divider sensing circuits which allows for maximizing the sensitivity [36].

5. Introduction of entropic sensing as an energy-efficient approach to real-time wireless sensing [37].

6. Introduction of Instantaneous Entropy (IE) as a way to quantify the rate of accumulating self-information in a time-series. Moreover, introduced entropic filtering as a down-sampling method for time-series in real-time [37].

7. Derivation of an iterative formula to efficiently compute Shannon's entropy for time-series which can be implemented on resource-limited hardware [37].

\subsection{Industrial Contributions}

1. The design and development of a low-cost, rugged SN to monitor the teeth adapters of a mining shovel. The SN carried nine sensors providing comprehensive situational awareness and redundancy for reliable proximity sensing to 
monitor mechanical integrity. The packaging was able to withstand an 8 ton impact force without loss in functionality while having minimal attenuation to the wireless signal through the exclusive use of low-permittivity materials [38].

2. Development of an integrated system to monitor the teeth adapters of an electric rope shovel allows Syncrude Canada Ltd. to reduce the probability of having stray metal in the ore and hence, reduce damaged crusher incidents and downtime. The solution combined a LoRa ${ }^{\mathrm{TM}}[29] \mathrm{WSN}$ with a cloud-based back-end to facilitate real-time off site monitoring and enable sensory data analysis.

3. Field testing the developed system on an operational shovel in an oil-sand mine (Chapter 4).

\subsection{Thesis Organization}

The thesis is organized as follows. Chapter 2 presents background. Chapter 3 describes the system's hardware design and all its components in detail. Chapter 4 discusses the implementation and field tests. Chapter 5 presents the topic of entropic sensing, develops the novel approach with an implementation and discusses its simulation results. Finally, Chapter 6 concludes the thesis and gives directions for future work. 


\section{Chapter 2}

\section{Background}

In this chapter, we build some background about hardware platforms in general and wireless sensors in particular. We explain the major components of an SN and describe their functional roles. Then, we cover previous related work in monitoring GET and discussed the two approaches followed in the industry to develop a working solution. Finally we discuss our adoption for one approach over the other in light of the application at hand.

\subsection{Hardware Platforms}

A Hardware Platform (HP) is a generic piece of hardware that is intended to host a wide range of analog and digital sensors depending on the given application. The sensors plus the HP is what we refer to as the $\mathrm{SN}$. In this section, we give an overview of HPs in the literature. Furthermore, we discuss the reason for designing our own HP instead of adopting a previous design.

Arkas [39] was designed to monitor snowfall. It uses an ultrasonic sensor wired to the HP, which connects back to a server through Zigbee at $2.4 \mathrm{GHz}$. The Arkas is intended for open-air environments, and its dimensions are $6.5 \times 6.5 \times 2.5 \mathrm{~cm}$. This 
HP supported up to seven analog and 16 digital inputs. The sensor is meant to have its own package and connect externally to the HP's input ports.

A low-power SN was developed in [40] for environmental monitoring. A ZigBee transceiver working at $2.5 \mathrm{GHz}$ was used, a second version was developed using the 868 $\mathrm{MHz}$ band. The system had two voltage regulators, which caused an efficiency drop in terms of power consumption. The sensors utilized were digitally interfaced to the controller using Maxim's proprietary 1-Wire protocol. The system was implemented and partially deployed with a reported three $\mathrm{km}$ range and a battery lifetime of more than three years with a bulky $10 \mathrm{Ah}$ battery. Again, an open-air environment was assumed for this HP.

In [41], an HP intended for instrumentation and predictive maintenance. The implementation employed inertial and position sensors. The HP utilized standard RF interfaces (e.g., Wi-Fi, Bluetooth). The hardware design consisted of a generic part, and external sensors to be wired as needed by the given application.

The RoSe is an application-specific SN rather than a generic HP [42]. The RoSe is a good example of a robust ultra-low-power SN. It operated at a sub GHz band. The work in [42] represents a well-thought-out design process and a rigorously tested product. The RoSe introduced the concept of an overmolded antenna housing and highlighted components that cannot withstand the injection molding process. The RoSe was designed for food monitoring applications which assumes an open-air environment around the antenna. The transceiver used (Semtech: SX1211) had a maximum output power of $12.5 \mathrm{dBm}$.

The work in [43], describes an SN employing a RFID energy harvesting technology to power its components when interrogated by an RFID reader. The SN utilized a 
printed dipole antenna to harvest RF energy, and assisted by a dynamic impedance matching network. The interrogation range was up to $1.5 \mathrm{~m}$, which is suitable for passive RFID applications but, insufficient for WSNs.

The authors in [44] have demonstrated an integrated System on Chip (SoC) solution to minimize power consumption. Their WiseNet node architecture has achieved a low level of power consumption down to $1 \mathrm{~mW}$ in receive mode with a $-95 \mathrm{dBm}$ sensitivity at $24 \mathrm{Kbps}$. The goal was to introduce a generic SoC for designing energyefficient SNs. The transmitter supported some of the Industrial Scientific Medical (ISM) band frequencies (433 and $868 \mathrm{MHz}$ ) and had an output power up to $10 \mathrm{dBm}$. Sprouts [45] [46] is an HP offering a multitude of hardware features designed specifically for industrial monitoring. A Plug and Play $(\mathrm{PnP})$ protocol [47] enables interchangeable sensing modules for different applications. An energy harvesting module with an optional backup battery for an extended lifetime. A remote triggering circuit for the transceiver to reduce its active time and contribute to the energy budget of the SN. A custom patch antenna, tuned to the SN's metallic enclosure [48]. A stackable circuit board, allowing for extending functionality. Figure 2.1 shows the hardware architecture of Sprouts. 


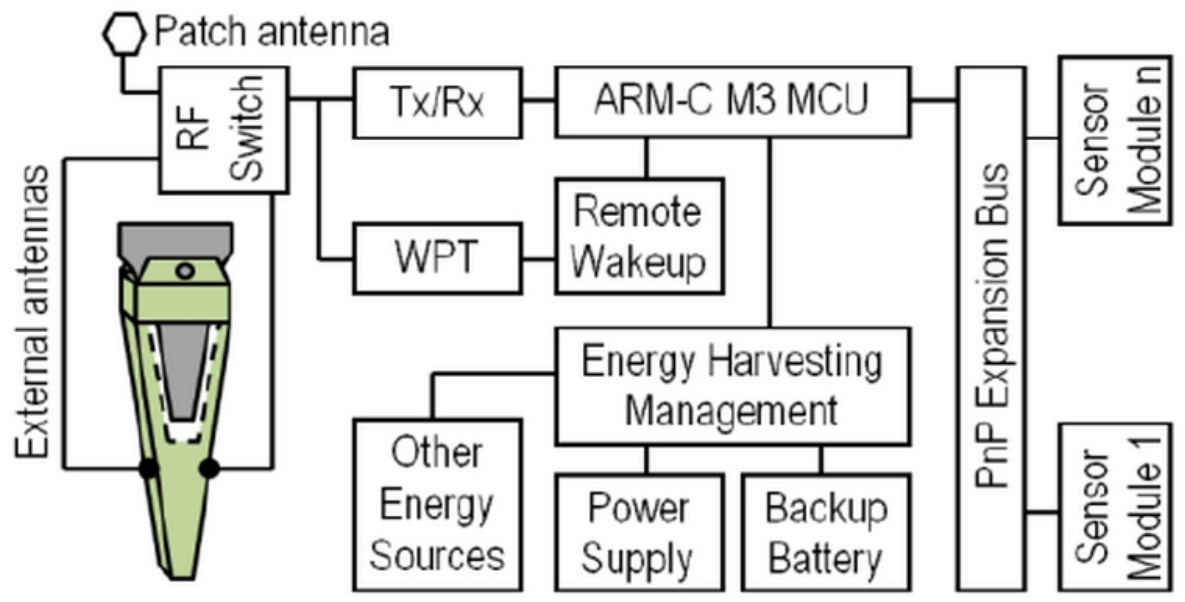

Figure 2.1: Sprouts hardware architecture. Reproduced with permission from [49].

Sprouts was designed to monitor a vibrating screen in an oil sands mine [50]. Later, it was framed as general-purpose HP for IoT . Sprouts was proposed to be used for monitoring GET [51]. Sprouts has hardware features needed for industrial monitoring applications. In Sprouts, integrating the microcontroller and the transceiver helped reduce its form factor; however, other applications may dictate the decoupling of the control and the communication module.

When it comes to different application domains, several design constraints can change. Consequently, a different SN will emerge from the design cycle. This is obvious in [52], where the requirements for multimedia applications have dictated the use of a power-demanding processor chip and a high bit-rate transceiver (Bluetooth). Industrial WSNs with a low data rate requirement will not benefit from the platform presented in [52].

For monitoring GET, the hardware requirements of the suitable SN will render existing platforms inadequate. For example, several SNs presented in the literature assume an open-air environment, and some SNs have a large form factor incapable 
of fitting into confined cavities. Furthermore, they cannot withstand harsh operating conditions.

As seen in the reviewed work, SNs are custom developed for each industrial application. For example, to monitor the impeller wear for a centrifugal pump [53], to

count the grains in a seed drill [54], monitor water pipelines [55], aid search and rescue robots in hazardous situations [56], or monitor axial tension in the bolts of a steel structures [57]. Numerous other examples can be added to the list; however, we have not encountered any record of an SN repurposed for a different application domain. This is in line with the arguments in [11], which asserted the need for custom-designed SNs in each application. Thus, the claim that an HP will satisfy the requirements of any application is unattainable with current technologies. Such a claim may be attainable in subdomains of applications with specific definitions for their requirements and operating conditions.

\subsubsection{Hardware Architecture}

The typical internal hardware architecture of an SN is composed of four units as shown in Figure 2.2. The illustrated units provide the four core functions of any SN. Some implementations may utilize chips that integrate two or more units to create a smallsize design, while other implementations segregate the units to introduce modularity and streamline future hardware and firmware development. In this section, we will introduce each unit in detail and discuss its currently available examples. 


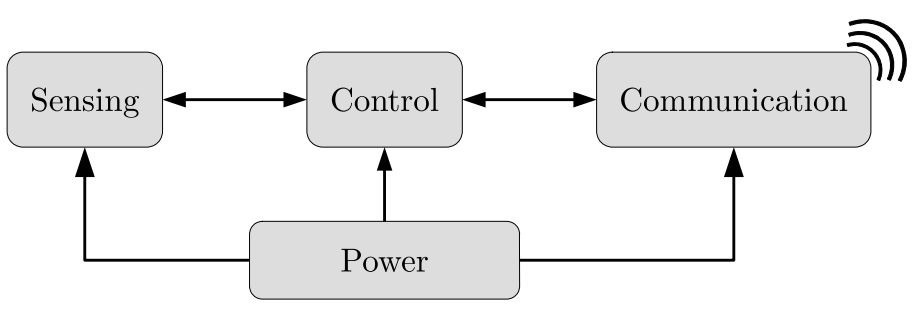

Figure 2.2: The block diagram of a typical sensor node.

\section{Sensing}

The sensing unit provides the sensory data dictated by the application requirements. It consists of transducers and their signal conditioning circuit. A transducer is a device that converts a given physical quantity to an electrical signal. The signal conditioning circuit is responsible for interfacing the special electrical output of the transducer to a standard input/output port on the microcontroller.

The sensors in this unit has to be suitable for wireless sensing applications in terms of their power consumption, size, and complexity. Examples of sensors include temperature, pressure, humidity, touch, light, motion, vibration, proximity, gas, smoke, position, tilt, strain, magnetic field, ultrasonic, infrared, flow, and liquid level.

\section{Control}

The control unit is practically a microcontroller. A typical microcontroller consists of a processing core, memory, a timing circuit, and some peripherals.

The processing core takes care of executing firmware instructions. It consists of an Arithmetic and Logic Unit (ALU), a Control Unit (CU), a communication bus, and a set of registers. The majority of available core architectures are proprietary, which means writing a portable firmware is almost impossible, however, there has 
been some recent effort to release an open source processor architecture called RISC$\mathrm{V}$ [58] (pronounced risc five), which started initially for desktop processors but is expected to propagate into the domain of microcontrollers.

The memory is divided into three main types. First, the flash memory, which is non-volatile and fast, hence used for storing the firmware. Second, the Random Access Memory (RAM), which is volatile and fast, hence used only in run-time because it cannot retain data in shutdown. Third, the Electrically Erasable Programmable Read-only Memory (EEPROM), which is non-volatile and slow, hence used for runtime data such as settings, parameters, and calibration data.

The timing circuit consists of one or two external oscillators and an internal clock distribution network. microcontrollers with two external oscillators use one for the normal active mode and use the other with lower frequency in power-saving sleep modes. The internal network takes care of clock division and distribution to properly trigger different parts of the system and maintain synchronization.

Peripherals are the microcontroller window to the outside world. Each microcontroller has its own set of peripherals which makes it suitable for certain applications. Examples of common peripherals include General Purpose Input/Output (GPIO) ports, Analog Digital Converter (ADC), comparator, Pulse Width Modulation (PWM) driver, and communication buses such as the Inter-Integrated Circuit (I2C) bus, the Serial Peripheral Interface (SPI) bus, and the Universal Asynchronous Receiver Transmitter (UART) bus.

The peripherals may include components that can support other core functions in the SN such as sensors (e.g., temperature, supply voltage) and even an integrated wireless transceiver. 


\section{Communication}

The communication unit is basically a wireless transceiver which supports one or more communication standards. The transceiver usually needs its own oscillator and requires some external components such as the balun circuit, the RF switch, the matching circuit and the antenna.

The balun circuit converts balanced differential signals to unbalanced single-ended signals. The RF switch enables the transceiver to switch between receive and transmit modes. The matching circuit lies right before the antenna to match its input impedance to the output impedance of the transceiver circuit for reducing signal reflection by extension internal power losses. The antenna is the radiating element which sends or receives propagating Electromagnetic (EM) waves within one or more specific frequency bands.

\section{Power}

The power unit is responsible for providing all components with enough power to run smoothly in any firmware dictated configuration, and enough energy to satisfy the lifetime requirement of the SN.

This unit is usually a storage device or an energy harvesting device or a combination of both. The dominant examples of a storage device is a battery. Examples

of energy harvesting devices include RF energy harvesting circuits [45], Photovoltaic (PV) cells, EM induction coils, thermoelectric peltier modules, and piezoelectric materials. 


\subsection{Monitoring Ground Engaging Tools}

There has been a profound interest in monitoring GET for construction, agricultural, and mining equipment. Worth mentioning that commercial interest in such a solution is highlighted by the number of patents reviewed in this section. Currently, two distinct approaches exist for monitoring GET [59]: image-based and wireless sensing solutions. In this section, we will go over both methods and review previous works in each method.

\subsubsection{Image-Based Solutions}

Image-based solutions employ several types of imaging technologies. Examples include visible light,infrared, and laser-imaging, detection, and ranging (LIDAR).

The invention in [60] utilized a thermal imaging camera to monitor thermal inserts inside the ground engaging tool. The inserts were embedded in the tool to provide significant thermal contrast for the camera to detect the tool's presence and wear level.

The inventions in [61-63] use a set of image sensors (e.g., LIDAR, camera) mounted at different viewing angles to the ground engaging tool to capture real-time 3D data and compare it with a pre-loaded 3D model to estimate the tool's wear level and detect its loss.

The typical image-based method employs a video camera assisted with an imageprocessing algorithm that can process video frames in real-time. A good example of a commercial product utilizing this method is presented in [64]. More recently,

the implementation of such a method was reported in academic research [65]. The solution in [65] involved the typical setup and utilized a neural network to process 
the frames in real-time. The system detected the shovel's bucket, the material inside, and the teeth with $96 \%$ accuracy. Worth mentioning that poor weather conditions were not considered in the study.

In [66], an offline method is presented where a technician would take photos of the ground engaging tool when the equipment is idle. The photo is uploaded to a cloud server for processing, results returned to the mobile application in the form of an estimate for the tool's end of life.

Even with enhanced image processing techniques, the inherent variability in the visual scene (lighting, weather conditions, type of ore) leaves the camera-based approach prone to errors. Furthermore, the need for machine learning in this method introduces a significant cost to develop, train, and maintain its software even after deployment. Also, the image-processing overhead can be resource-demanding in terms of computing power. However, this monitoring method is passive, which means the installation cost is reduced since it does not require any intervention with the ground engaging tool. On the other hand, the image-processing algorithm must be redeveloped and tuned for each particular piece of equipment.

\subsubsection{Wireless Sensing Solutions}

Wireless sensing solutions use WSNs to collect measurements directly from the ground engaging tool. Usually, the WSN in this solution type is configured in a single-hop star topology to reduce latency. In this section, we will discuss previous work in the wireless sensing approach and highlight its viability.

In [51] and [49] [67], the use of Sprouts was proposed for monitoring GET. However, the actual implementation had not been developed. While such works presented 
a good feasibility analysis, some aspects of the proposed implementation were impractical. For example, in [51], the SN required a hole drilled in the shovel tooth to install the antenna. The hole depth and diameter are hard to achieve with current machining or casting technologies. Also, a hole in the tooth is undesired since it may compromise its structural integrity and shorten its expected lifetime.

The invention in [68] comprises of an $\mathrm{SN}$ embedded in a recess in the ground engaging tool's structure and open to the exterior. While common sense would predict severe damage to the $\mathrm{SN}$ in such a location, it is safe and has the advantage of efficiently radiating EM waves. Thus, the SN in [68] can easily use Bluetooth or a similar technology to communicate with its sink. This patent is now a commercial product [69].

In [70], the inventors present a WSN with an SN equipped with an ultrasonic sensor to monitor the ground engaging tool's wear-level on agricultural graders, bulldozers, or earth processing equipment in general.

The invention in [71] is perhaps the closest previous work to the work in this thesis. While it utilizes an SN with a proximity sensor.

For monitoring GET, wireless sensing solutions require far less real-time data processing compared to image-based solutions. While WSNs are not passively deployed, they can monitor any ground engaging tool and, by extension, any industrial asset using the same system. The only equipment-specific part of the network is the SN.

We steered our efforts towards wireless sensing because this method directly measures the state of the ground engaging tool. Thus, it has the potential to monitor the tool with more certainty compared to image-based methods. Furthermore, embedding an SN inside the tool would offer the opportunity to monitor more variables 
directly such as internal temperature, mechanical shocks, acceleration, wear-level, and equipment utilization. Finally, with wireless sensing, the system will be decoupled from poor weather and low-light conditions.

\subsection{Summary}

In this chapter, we introduced some background for hardware platforms and SNs. we over-viewed the previous related work in monitoring GET, and concluded our approach for developing a working solution. 


\section{Chapter 3}

\section{System Design}

In this chapter, we will introduce our case study, explain the application requirements, and discuss the system design in light of such requirements. The discussion will cove all system components and will dive deeply into the SN's design including the application specific sensors developed in this work.

Mining oil sands, is a complex process that requires numerous critical factors to be in sync for the process to stay economically viable [72]. It starts with massive electric rope shovels (payload $\approx 100$ metric ton) digging the ore out of the ground, as shown in Figure 3.1. Then a large fleet of hauler trucks carries the ore back to the processing plant where the rock crusher sits at its inlet. The crusher's function is to grind the mined rocks down to manageable sizes. The crusher is operational around the clock to feed the plant continuously. Thus, its downtime is a direct cause of costly production cuts. 

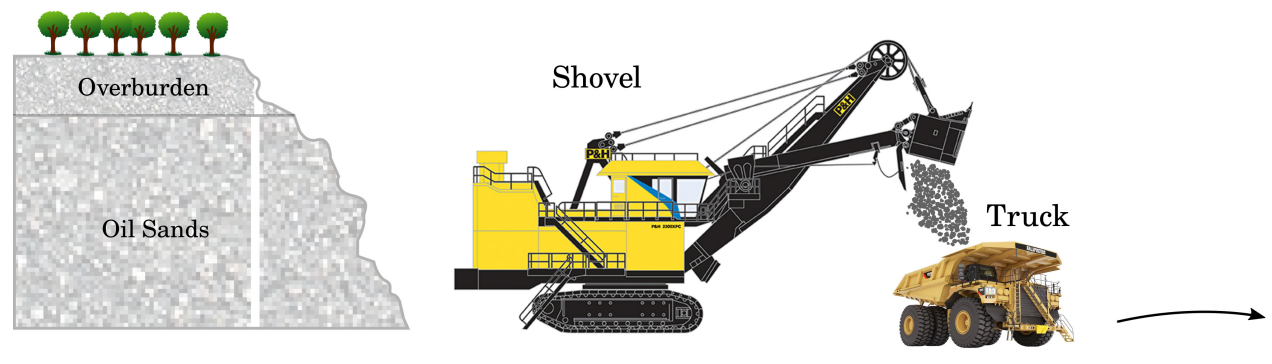

Crusher

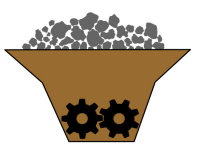

Figure 3.1: The first stage in an open-pit oil sands mining process. 
One of the primary reasons for the crusher's downtime is the unnoticed presence of scrap steel chunks in the ore that can jam the crusher causing serious damage. Often during the dig, a shovel adapter/tooth will break, fall in the hauler truck, and end up in the inlet of the crusher, jamming it and causing significant maintenance bills, a few hours of halted production and in some cases, human injury [33]. Scrap steel can damage other equipment as well. For example, at one of the oil sand mines in Fort McMurray, Alberta, Canada, a detached tooth got stuck on a conveyor belt, causing nearly a kilometre long rip in the belt, which incurred a huge maintenance bill that could have been avoided if the tooth was promptly retrieved right after it became detached. In our case, the projected cost of monitoring GET with a WSN is significantly lower than the cost of potential damages resulting from their accidental detachment.

The targeted shovel has a bucket with nine adapters. Each adapter has two arms to sandwich the bucket's lip. Each arm has an internal cavity, as shown in Figure 3.2. As reported by mining field technicians, adapters break at the arms. Thus, an $\mathrm{SN}$ is installed in each arm to monitor its integrity, bringing the total number of SNs to 18 . 


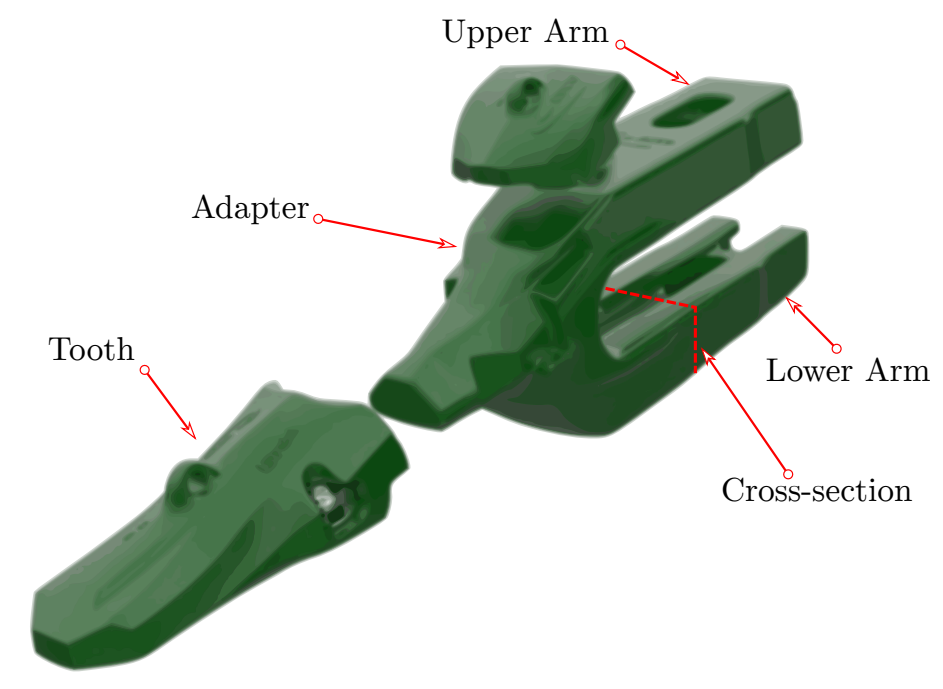

Figure 3.2: The adapter/tooth assembly of the designated electric rope shovel.

When an adapter breaks, it could end up either buried underground, exposed on the ground, or in a hauler truck, depending on one of four possible scenarios as listed in Table 3.1. The SN must be able to identify these scenarios and communicate its status accurately at all times.

Table 3.1: Breakdown of adapter detachment events.

\begin{tabular}{|l|c|c|}
\hline & Bucket & Adapter \\
\hline Scenario 1 & Engaging ground & Buried underground \\
\hline Scenario 2 & Engaging ground & Exposed on the ground \\
\hline Scenario 3 & Lifting payload & Exposed on the ground \\
\hline Scenario 4 & Dumping payload & In the hauler truck \\
\hline
\end{tabular}


The system, as shown in Figure 3.3, is a single hop star topology WSN. The SNs are installed inside the adapters. The sink is mounted on the operator's cabin. Other sink nodes are installed along the truck's route to the crusher, as shown in Figure 3.5, to pick up any signals from a detached adapter before it reaches the crusher.

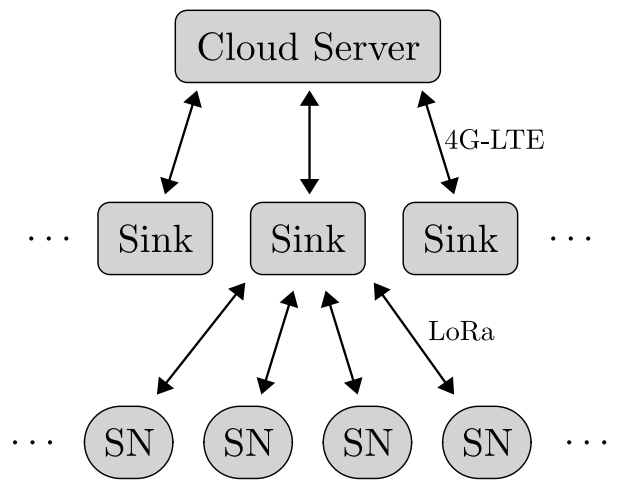

Figure 3.3: Network diagram.

Each sink is connected to a back-end cloud server as shown in Figure 3.4. The cloud server hosts a dashboard application [73] which enables offsite users to access real-time data and create a data repository to facilitate advanced analytics of the industrial process. Local system alarms are not triggered by the cloud server but are instead handled by sink nodes to avoid basing the reliability of time-critical alarms on the cellular connection.

The typical design challenges of SNs are: power consumption, wireless link performance, mechanical robustness, and cost. Addressing all challenges concurrently will often lead to conflicting design requirements. Establishing a delicate balance between such requirements is simply the key to a successful hardware design.

The hardware design of our SN follows the typical architecture of SNs in Figure 2.2. The architecture of our $\mathrm{SN}$ is shown in detail in Figure 3.6. However, in energy 


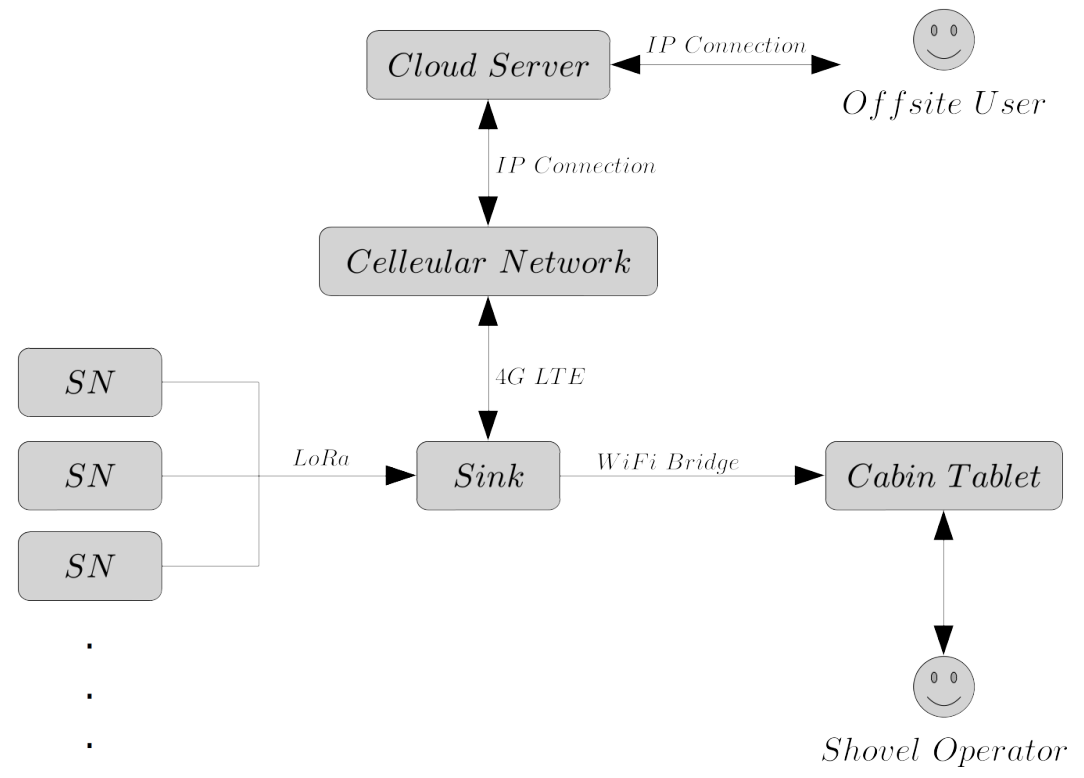

Figure 3.4: System block diagram.

harvesting applications, the flow of power and data between the units is different from what is illustrated in Figure 2.2. In this section, we will discuss the design of each unit in detail.

The design requirements for the SN are divided into categories; each decomposes into several lower-level requirements in a tree structure. A summary of the tree is shown in Figure 3.7. The tree allowed for identifying conflicts quickly and helped in guiding mitigation strategies during design.

The essential function of the $\mathrm{SN}$ is to monitor the proximity of the adapter to the shovel's bucket during active operations. Whenever an adapter is detached, the SN will notify the sink and, in turn, the operator in real-time.

Developing proper design requirements is an iterative process that benefits significantly from experience. Designing for better performance while minimizing and/or balancing conflicts requires proper technical competency and up-to-date knowledge 


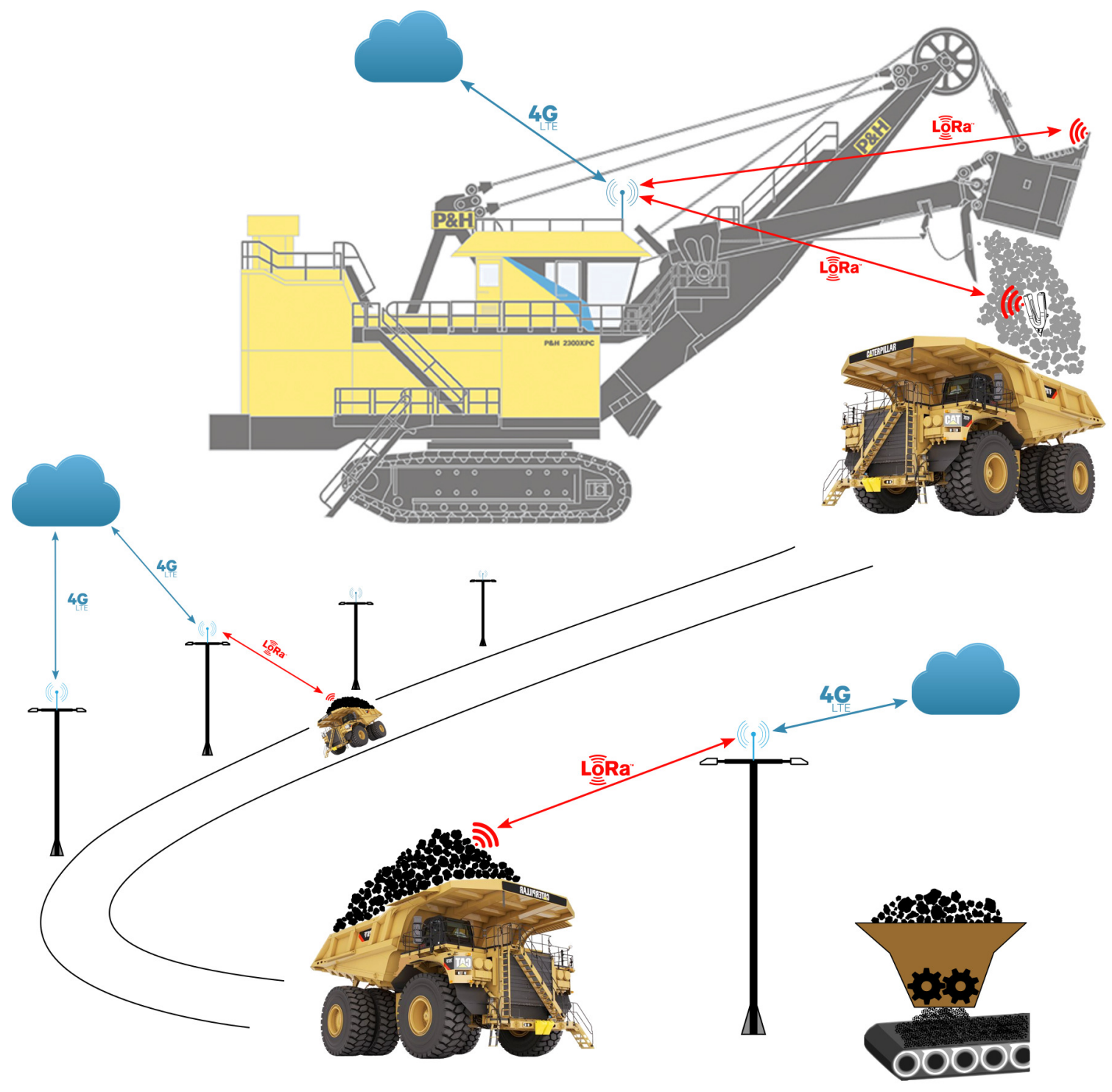

Figure 3.5: System overview. 


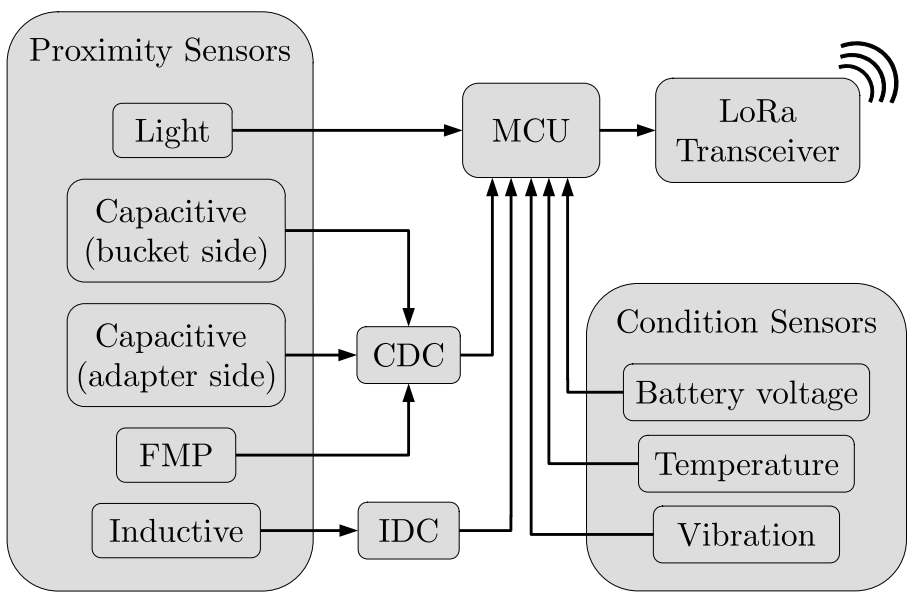

Figure 3.6: A detailed block diagram of our sensor node.

of available components in the market. While the actual tree had many conflicting requirements, the summary in Figure 3.7 shows only three. As an example, we will discuss conflicts 2 and 3. To maximize the battery capacity as in requirement (Req) 3.1.2, we need to add as many cells as possible without over-sizing the $\mathrm{SN}$ as in Rq 1.2, which gave rise to conflict 2. Further on, conflict 2 sparked Rq 3.1.1, which narrowed down the choices of battery chemistry and created conflict 3. Consequently, we chose a battery chemistry (Lithium-thionyl Chloride) that provided high energy density while withstanding temperatures in $\mathrm{Rq} 2.1$. 
1 Core requirements

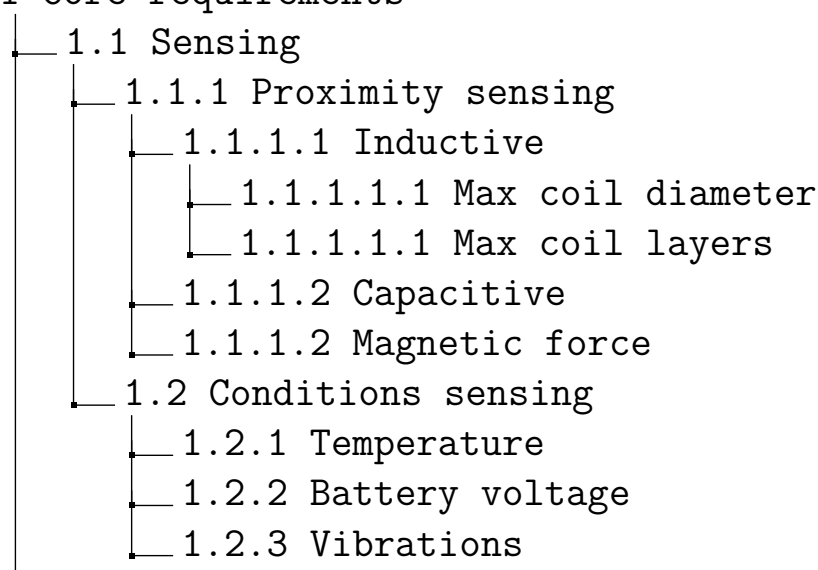

1.2 Size has to fit the cavity...

conflict 2

- 1.3 Communications

-1.3.1 Min latency

1.3.2 Min packet loss ratio

1.3.3 Max range (> $25 \mathrm{~m}$ )

1.3.3.1 Antenna matching

1.3.3.2 Max $\mathrm{Tx}$ power ..................................... 1

2 Mechanical robustness

2.1 Temperature tolerance: -40 to $85^{\circ} \mathrm{C}$.

2.2 Vibration tolerance

-2.3 Mechanical shock tolerance

3 Lifetime (> 1 year)

- 3.1 Battery

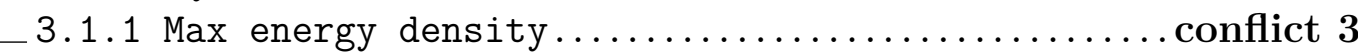

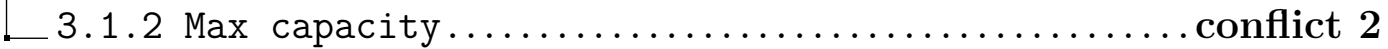

3.2 Power consumption

3.2.1 Min $\mathrm{Tx}$ power ......................................... 1 3.2.2 Min active time

Figure 3.7: A summary of the sensor node design requirements tree. "Max" and "Min" stand for maximize and minimize respectively. Tx stands for transmit. 


\subsection{Sink}

The sink houses several hardware modules, as shown in Figure 3.8. The Raspberry Pi 4 (RPi) is a Single Board Computer (SBC) running Linux and serves as the central manager of all the other modules. It connects over Wi-Fi to the cabin-mounted tablet. A Hardware Attached on Top (HAT) is a standard hardware module that can be stacked on top of the RPi. In this setup, the RPi has 3 HATs: First, the 4G-LTE HAT (Quectel EC25-A), which houses a data-enabled sim-card [74] and allows for a connection through cellular networks to the back-end server. Second, the uninterruptible power supply (Pi Supply: PIS-0212) is supported by a 5 Ah lithiumion battery to ensure the sink will be online (for a few hours) in case of a power interruption, this allows for backing up any queued data and safely shuts down the node until power is restored. Third, the LoRa ${ }^{\mathrm{TM}}$ Gateway (RisingHF: RHF0M301) connects to all the SNs over multiple channels in the Industrial, Scientific \& Medical (ISM) band of 902-928 MHz. All the hardware was housed in a weatherproof (IP67) box. 


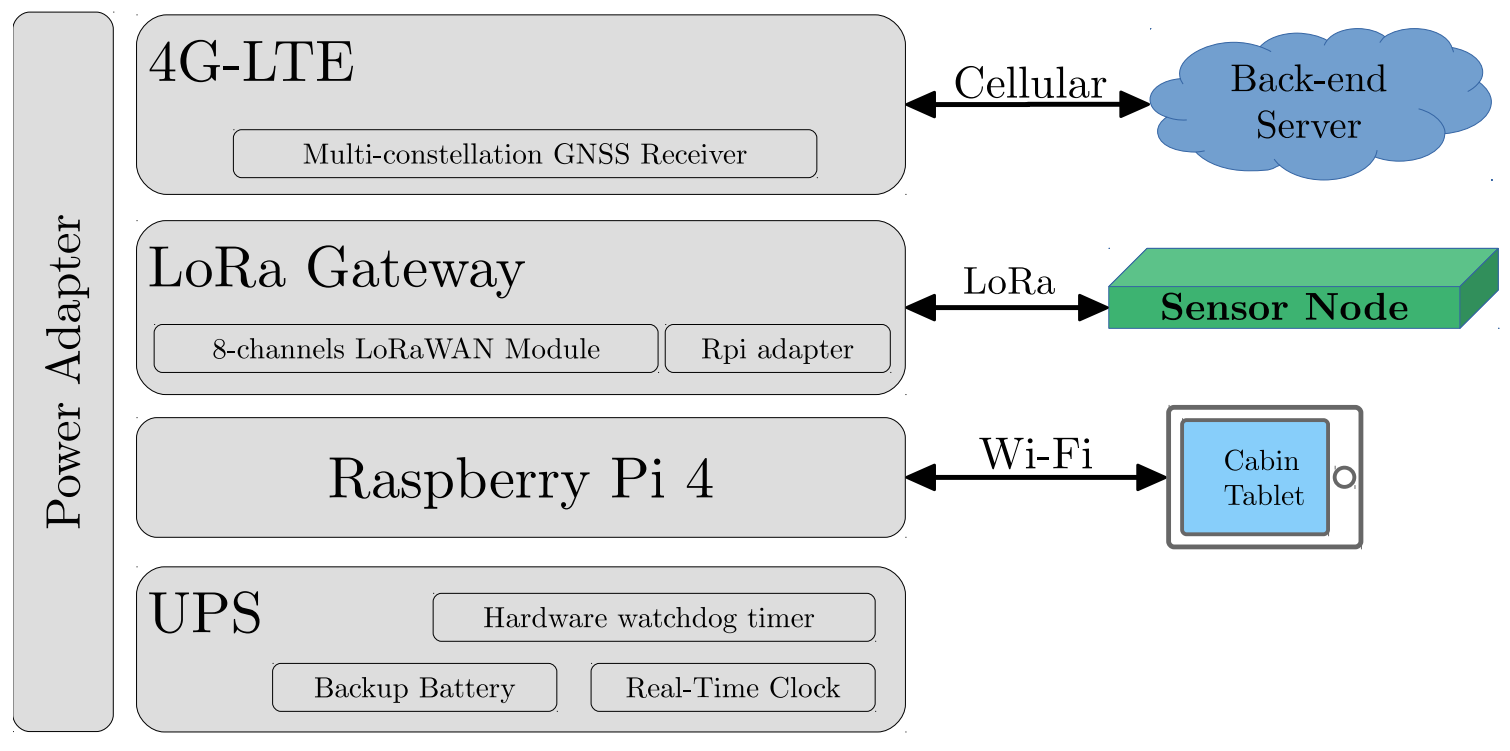

Figure 3.8: Block diagram of the sink hardware setup.

\subsection{Control}

The control unit is an ultra-low-power microcontroller (Texas Instruments: MSP432P401R).

It connects to all the sensors through the $\mathrm{I} 2 \mathrm{C}$ bus, and connects to the $\mathrm{LoRa}^{\mathrm{TM}}$ transceiver through the Serial Peripheral Interface (SPI) bus. It is programmed through the JTAG interface and utilizes two oscillators; a $16 \mathrm{MHz}$ oscillator for the active mode, and a $32.768 \mathrm{kHz}$ for the low-power mode.

The firmware handles low-level sensing functions and the MAC protocol managing the LoRa ${ }^{\mathrm{TM}}$ transceiver. More on the MAC protocol in Section 3.4.5. The setup part of the firmware configures the sensors and the transceiver by loading preset data to their internal registers. The firmware use a Real-Time Operating System (RTOS) to schedule different tasks and handle interrupts.

The design allowed the controller to shut down all other components when needed and go into a deep sleep mode to save energy (current consumption down to $150 \mathrm{nA}$ ) 
when the shovel is idle. Usually, the long wake-up time (1.1 ms) becomes a concern when going into the deep sleep mode; however, in our case, the shovel takes a lot more time (tens of seconds) to transition from idle to active.

\subsection{Power}

Req 3, in Figure 3.7 dictated a constrained energy budget, given the limited space inside the cavity. Consequently, a number of energy-saving measures were taken.

On the communication side, as aforementioned, we use a custom energy-efficient MAC protocol [1] [75]. Additionally, firmware optimization allowed for considerable savings in the energy budget. To quantify the energy demand in the design stage, we identified the duty cycle of the $\mathrm{SN}$, which is composed of the following tasks: wake up, collect sensory data, activate the radio, transmit a data packet, and sleep.

On the storage side, we balance Req 3.1.1 and Req 2.1 in Figure 3.7. Req 3.1.1 was invoked by the limited room inside the adapter's cavity. Req 2.1 was introduced because of the very low operating temperature at the oil-sand mine in Fort McMurray, Alberta, Canada (down to $-40^{\circ} \mathrm{C}$ [34]). After considering size and cost constraints, we chose an AA-size battery (SAFT: LS14500) with a rated capacity of 2.6 Ah (at 3.6 V). The design could accommodate up to nine parallel cells inside the SN's package. This boosts the maximum continuous current allowed (up to $450 \mathrm{~mA}$ ), and the total rated energy capacity (up to $301.48 \mathrm{~kJ}$ ). While, the chosen battery can stay functional in frigid temperatures, its capacity drops to almost a third of its rated value at room temperature. Thus in order to estimate the usable energy capacity, we assumed a conservative factor of safety of 0.5 , which brought the budgeted energy capacity down to $151.74 \mathrm{~kJ}$. 
Table 3.2: Rated power consumption of devices onboard PCB-full (@ $V_{\text {supply }}=3.6$ $\mathrm{V})$.

\begin{tabular}{|c|c|c|c|c|}
\hline \multicolumn{2}{|r|}{ Devices } & Active mode & Sleep mode & $\begin{array}{ll}\text { Deep } & \text { Sleep/ } \\
\text { Shutdown mode }\end{array}$ \\
\hline & Controller & $4.6 \mathrm{~mW}$ & $540 \mathrm{nW}$ & $90 \mathrm{nW}$ (@ LPM4.5) \\
\hline & Transceiver & Tx:425 mW, Rx:15 mW & $4.3 \mu \mathrm{W}$ & $576 \mathrm{nW}$ \\
\hline \multirow{4}{*}{ 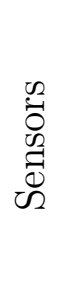 } & Capacitive & $10.8 \mathrm{~mW}$ & $216 \mu \mathrm{W}$ & $3.6 \mu \mathrm{W}$ \\
\hline & Inductive & $11 \mathrm{~mW}$ & $126 \mu \mathrm{W}$ & $3.6 \mu \mathrm{W}$ \\
\hline & Light & $341 \mu \mathrm{W}$ to $647 \mathrm{nW}$ & $\approx 0$ & $\approx 0$ \\
\hline & Accelerometer & $648 \mu \mathrm{W}$ to $180 \mu \mathrm{W}$ & $18 \mu \mathrm{W}$ & Not available \\
\hline
\end{tabular}

Finally, we simulated the energy budget using our measurements in Chapter 4, Section 4.4 while adjusting the frame rate to balance the budget and satisfy the lifetime requirement. The adjusted frame rate was estimated at 4.65 frames per minute. In other words, the SN can transmit a data packet every $13 \mathrm{~s}$ for at least one year. On the other hand, the shovel is expected to be idle a few times throughout the year, which will cause the SN to go into a deep sleep mode. This will increase the lifetime even more depending on the number of times the shovel is idle.

While the chosen battery cell balanced our energy budget, it came with some disadvantages. First, it contained toxic chemicals, which means the SN must be properly disposed of at the end of its lifetime. Second, it carried a risk for overheating, leaking or even exploding if shorted, which was the case in one of our impact tests for the SN when the testing weight dropped on the SN caused a bend in one of the pins in the programming connector, causing a short-circuit between the battery terminals. 


\subsection{Communication}

Industrial equipment is generally made of metallic parts (e.g., steel) for their high strength, ductility, and durability. These properties allow industrial equipment to withstand high levels of mechanical stress. However, while metals have numerous desirable mechanical properties, their high electrical conductivity causes reflection and attenuation to electromagnetic waves. This high electrical conductivity makes it hard to install wireless sensors inside metallic industrial equipment.

Machinery, equipment, and industrial assets with metallic enclosures and structures are prevalent in industrial settings, for example, pumps, turbines, metallic pipes, valves, combustion chambers, engines, pipes, chemical reactors, motors and robotic

arms. Their control systems must have some sort of feedback in order to stabilize and optimize their operation. As the future pushes for smarter and more automated equipment and machines, the need for transmitting wireless data from and to metallic enclosures becomes more pressing.

As shown in Figure 3.9, industrial enclosures can be divided into two types (based on their material): metallic and non-metallic. Metallic enclosures are made completely or partially from metals. Metallic enclosures pose a more significant challenge on the hardware design because of their high electrical conductivity. Further, enclosures can be sub-classified (based on the type of concealment) into two types: hermetic and non-hermetic.

Non-metallic enclosures are made entirely from non-metals. This type of enclosure (whether hermetic or non-hermetic) does not pose any challenges because arbitrary RF signals are able to propagate through these enclosures with minimal attenuation.

Hermetic Metallic (HM) enclosures are sealed and can be described as airtight. 


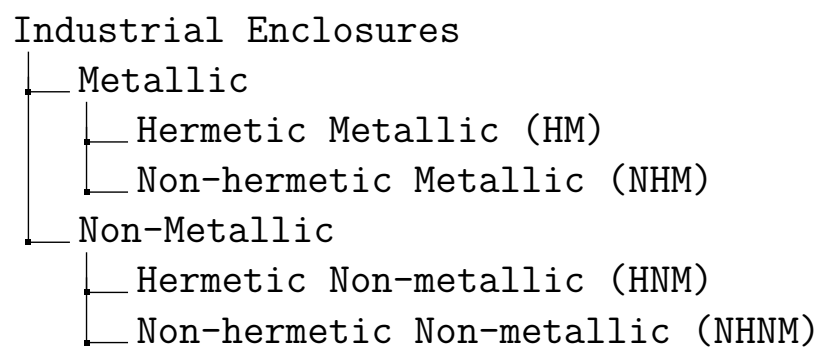

Figure 3.9: Classification of industrial enclosures according to their material and level of sealing.

In other words, the metallic structure provides no or negligible clearances between its metallic components. Examples of HM enclosures are: pumps, turbines, gas cylinders, and combustion engines. This type of enclosures is beyond the scope of this work because, wireless communications from within these enclosures cannot be achieved by a propagating electromagnetic wave, however, it requires different techniques [76], which are classified into either electromagnetic techniques (inductive coupling, capacitive coupling, and magnetic resonance coupling) or acoustic techniques.

Non-hermetic Metallic (NHM) enclosures are those with a low level of conductive concealment. They are often designed to provide mechanical, environmental, or chemical shielding from abusive conditions. Non-hermetic Metallic (NHM) enclosures have relatively large clearances between the metallic components of their structure. They can still be described as airtight if the containment structure is not fully metallic. Examples of NHM enclosures are shipping containers, refrigerators, and industrial ovens.

NHM enclosures are very common in countless industrial applications. This enclosure type can represent any industrial setting where the SN is surrounded mainly by metals but not from all directions. Thus, the assumption is that it is possible for the RF signal to propagate and reach its destination. In this work we investigate the 
propagation of RF signals transmitted by an SN from within an NHM enclosure.

Low frequencies (sub $\mathrm{GHz}$ ) hold the best potential in escaping industrial NHM enclosures because free-space path loss is directly proportional to the signal's frequency [77]. However, the lower the frequency, the larger the antenna, which is a design conflict that can be mitigated by application requirements.

\subsubsection{Approach}

For enabling EM waves to escape the enclosure with enough power to reach the destination reliably, there are two methodologies to that can be followed. The first one works by treating the metallic enclosure and its gaps as a resonant cavity [78]. This means several aspects of the design have to be optimized to a specific enclosure given its exact dimensions. This entails a custom antenna design considering its position and orientation inside the enclosure, and the frequency of operation $f_{o}$. If the SN were to be installed in a different enclosure, its performance would differ in an unanticipated manner. While this method allows the wave to escape the enclosure more efficiently (using less transmit power $P_{t}$ ), it only works for the predefined enclosure. In other words, the antenna design process will have to be repeated for any other enclosure.

The second method is more generic and applicable to almost all enclosures; however, it is more energy demanding. It starts by calculating the cutoff frequency $f_{c}$ (lowest resonant frequency) of the enclosure and its gap and choosing a higher operating frequency $f$ o for the platform. Then it is a matter of getting a transceiver with sufficient transmitting power that can balance the link budget at the required 
range $\mathrm{R}$ given the receiver's sensitivity $S_{\text {min }}$. This may come at the expense of increased energy consumption and reduced lifetime. Thus, the number of data packets transmitted $N_{p}$ and the size of data payloads should be optimized to balance the energy budget. Also, this method is more cost-effective since it is more generic and decoupled from a specific enclosure geometry.

The SN is located inside the adapter of the shovel tooth. According to our initial field investigations, most adapter failures occur along the cross-section of its arm, as shown in Figure 3.2. This means the SN inside the arm's cavity will be compromised in a such an event. This led us to require the $\mathrm{SN}$ to transmit its status update as frequently as allowed by the energy budget.

The sink is placed on top of the operator's cabin. The distance between the SN and sink is at most $25 \mathrm{~m}$ throughout the dynamic span of the bucket. Since the adapter is made of steel, the SN's inside its internal cavity will have a hard time emitting electromagnetic waves because the steel adapter behaves like a Faraday cage [79]. Modifying the structure to allow for efficient radiation was prohibited by the industrial partner over concerns of compromising the adapter's mechanical integrity. To satisfy $\mathrm{Rq} 1.3$ and establish a reliable wireless link, we exploited the dimensional clearances in the adapter's cavity, which was non-hermetic with gaps $(5 \rightarrow 10 \mathrm{~mm}$ ) along the edge of the adapter's arm, as shown in 3.15. These gaps weaken [79] the shielding effect of the steel cavity and allows the signal to escape the cavity with enough power to reach the sink reliably.

With the SN installed inside the shovel adapter, it is not an option to retrieve it midlife to replace its battery. Thus, its energy budget has to allow for a lifetime that matches or exceeds that of the adapter, which is approximately one year. 


\subsubsection{Experiment}

We designed an NHM enclosure model as shown in Figure 3.10 and Figure 3.11. The base and the cover were each machined from a single piece of steel. It has two identical gaps $(228.6 \times 1 \mathrm{~mm})$ on opposite sides. The depth of each gap is $76.2 \mathrm{~mm}$. A total of eight screw holes were threaded on the edges to secure the cover tightly to the base. The total weight of the model is $23 \mathrm{~kg}$. The dimensions of this model are chosen to mimic the cavity in the shovel's adapter in our case study. The gaps in the model are similar to those between the adapters and the shovel's bucket when the adapter is installed. The signal is received by an antenna mounted on top of the operator's cabin, which is about $25 \mathrm{~m}$ away from the moving bucket.

From a signal propagation standpoint, representing various industrial enclosures with one physical model is infeasible. However, a model representing an extreme RF shielding case could be used to set a lower bound for the performance of the SN. With a gap of only $1 \mathrm{~mm}$, this model has a significant shielding effect compared to typical industrial NHM enclosures, which usually have wider gaps and openings. Thus, the SN that proves to work for this particular enclosure is likely to work for other less concealed enclosures.

The modem chip selected for our platform was the Semtech SX127x. It incorporates a LoRa ${ }^{\mathrm{TM}}$ spread spectrum module that achieves $8 \mathrm{~dB}$ better sensitivity than using frequency shift keying (FSK) modulation at an equivalent data rate. For compatibility with existing systems, the selected chip provides the standard radio modulation and demodulation techniques (GFSK, FSK, OOK, and GMSK). LoRa ${ }^{\mathrm{TM}}$ uses the chirp spread spectrum technique. It also uses forward error coding in combination with whitening and interleaving in order to achieve this improved sensitivity. LoRa ${ }^{\mathrm{TM}}$ 
operates in the $433 \mathrm{MHz}, 868 \mathrm{MHz}$, and $915 \mathrm{MHz}$ bands, making it suitable for ISM bands in Europe and North America. LoRa ${ }^{\mathrm{TM}}$ is designed for LPWAN; however, in this work, we implement our own network stack to create the WSN. The chip also provides ease of access to multiple modulation settings, thus increasing flexibility of implementation, allowing the user to balance receiver sensitivity, power consumption and bit rate. This is crucial for optimizing the lifetime of our SN while ensuring a resilient communication link.

An experiment to test the communication link and quantify the received signal strength (RSS) is conducted. Data packets are sent from the platform inside the enclosure and received at the other end (without errors) and then used to quantify the RSS. The settings used in the experiment are listed in Table 3.3.

\section{Setup}

The LoRa ${ }^{\mathrm{TM}}$ transmitter is programmed to transmit with maximum power $(20 \mathrm{dBm})$. However, due to coupling losses and implementation imperfections, the actual measured power at the input of the antenna was $18.5 \mathrm{dBm}$. A custom-designed patch antenna of a $3 \mathrm{~dB}$ maximum gain was used. In a wide-open outdoor area, the platform was activated and placed inside the cavity of the enclosure. The lid was closed tightly using four corner screws. The enclosure was placed on a turntable with a degree scale. The receiver Yagi antenna (18 dB maximum gain) was placed horizontally $30 \mathrm{~m}$ away from the enclosure. Received Signal Strength (RSS) readings were collected with $10^{\circ}$ steps of angular rotation. 


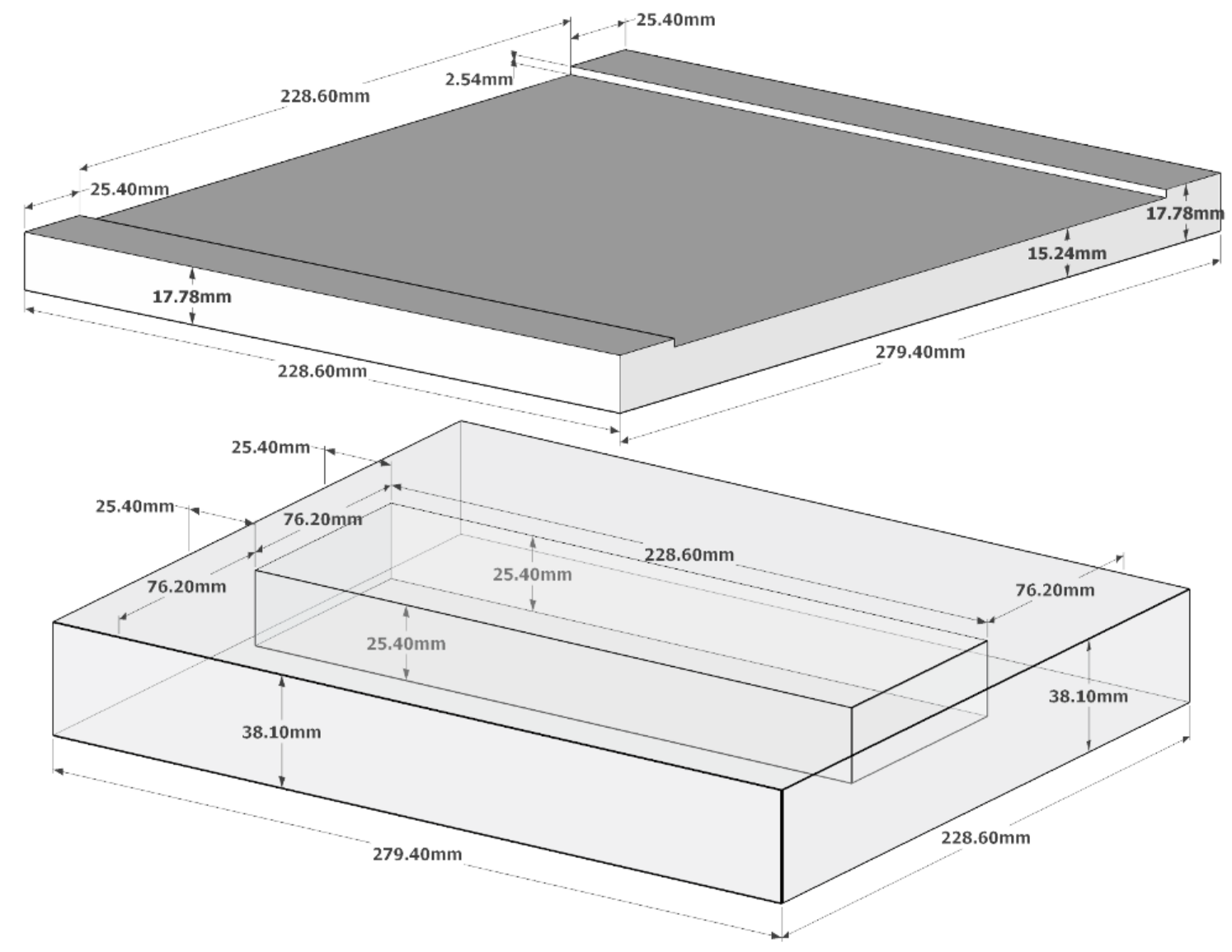

Figure 3.10: CAD drawing of the NHM enclosure.
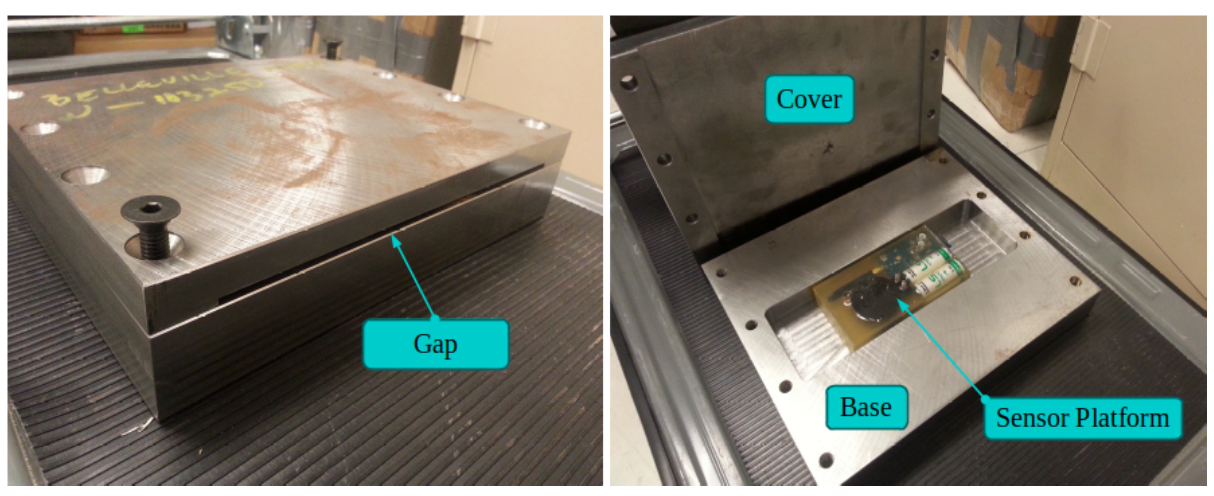

Figure 3.11: The NHM enclosure and the sensor platform inside its cavity. 

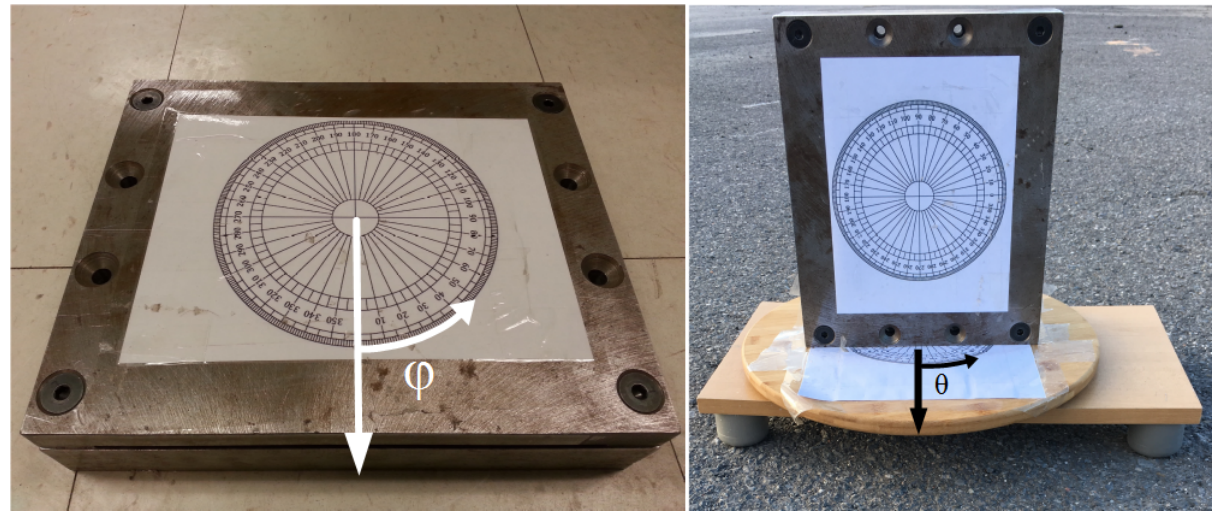

Figure 3.12: The orientation of the enclosure with respect to the angle of rotation in the horizontal and the vertical position.

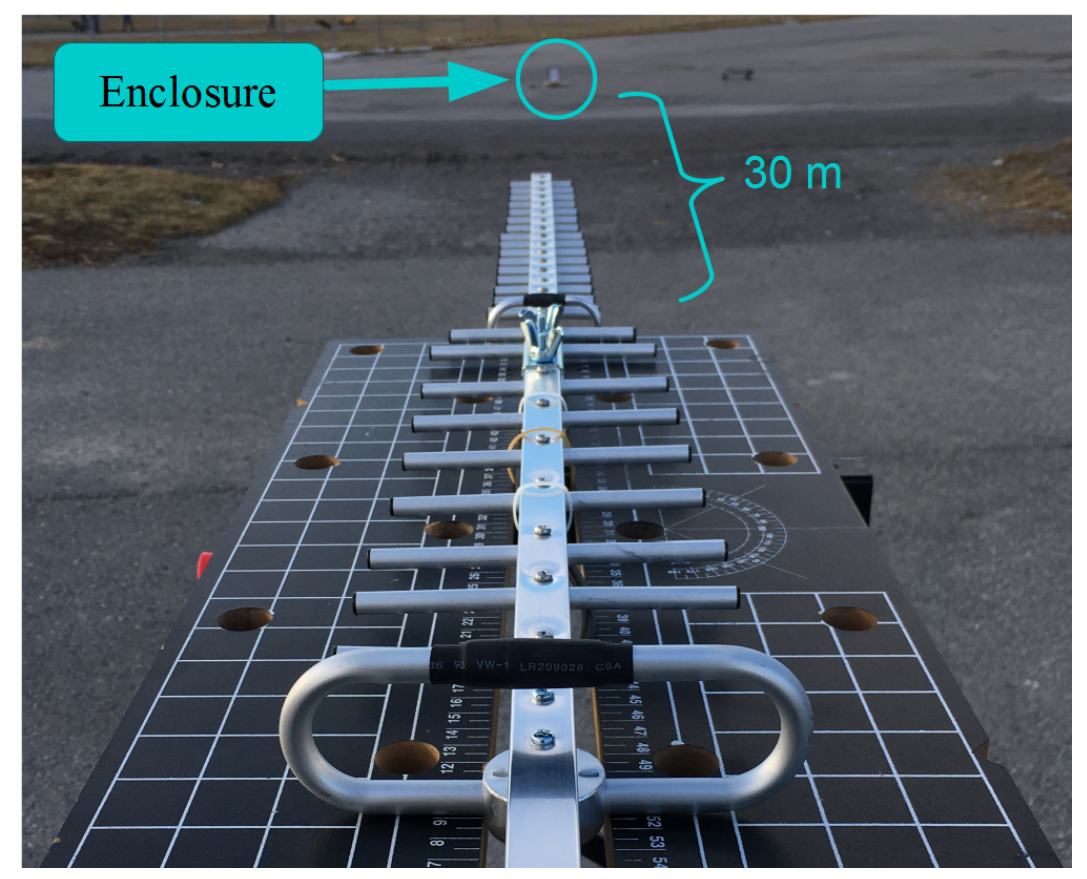

Figure 3.13: The experimental setup showing the Yagi antenna at the receiver side and the enclosure $30 \mathrm{~m}$ away. 


\section{Results}

The RSS readings from all angles around the enclosure are shown in Figure 3.14. Two sets of measurements are provided; one for when the enclosure is placed horizontally and the other when it was placed vertically, as shown in Figure 3.12. At each angle, 20 readings were collected, and their average is used in the plot. Confidence intervals are two times the standard deviation of all readings recorded at that angle. In other words, it includes $68 \%$ of the population of readings assuming a normal distribution.

As shown in Figure 3.14, orienting the enclosure vertically gives higher RSS readings over all the angles. The reason behind this observation comes back to the complex relationship between the antenna inside the enclosure and the structure of the enclosure because it exists in the reactive field of the antenna. The pattern of the RSS readings started to repeat itself at $180^{\circ}$. This is expected since the enclosure and the antenna inside the enclosure are symmetrical around the rotation axis. While the RSS readings are at a very low level, it is still within the link budget corresponding to the used settings $(151 \mathrm{~dB})$.

The extreme signal attenuation caused by the steel adapter called for a significant wireless link budget. Also, the data payload was very small and could be easily handled by a low data rate link. Consequently, LoRa ${ }^{\mathrm{TM}}$ was chosen because of its superior link budget at the cost of a low data rate link [80]. The subject of wireless communication from within a non-hermetic metallic enclosure is discussed further in a separate work [34].

A Yagi array was used with the LoRa ${ }^{\mathrm{TM}}$ transceiver of the sink. It improved the wireless link budget by about $14 \mathrm{~dB}$. The location of the sink relative to the bucket is shown in Figure 3.1. Thus, with a highly sensitive receiver (down to $-142.5 \mathrm{dBm}$ ) 


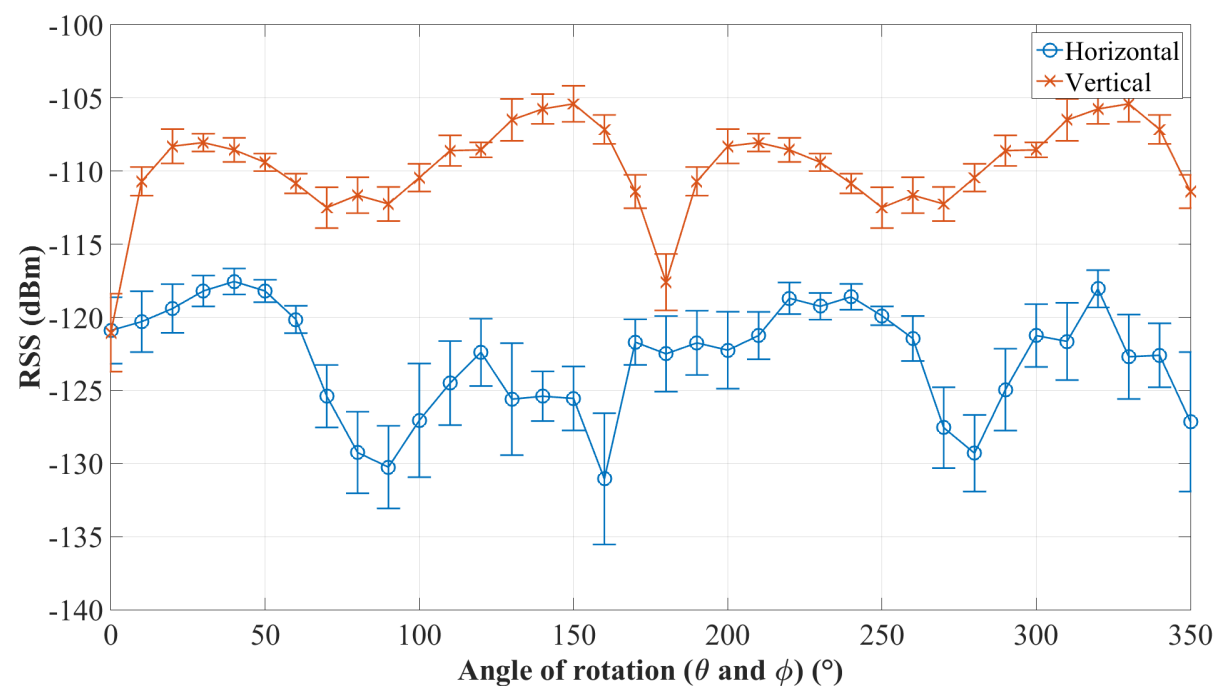

Figure 3.14: Received signal strength at $30 \mathrm{~m}$ range for all angles around the enclosure when placed horizontally and vertically.

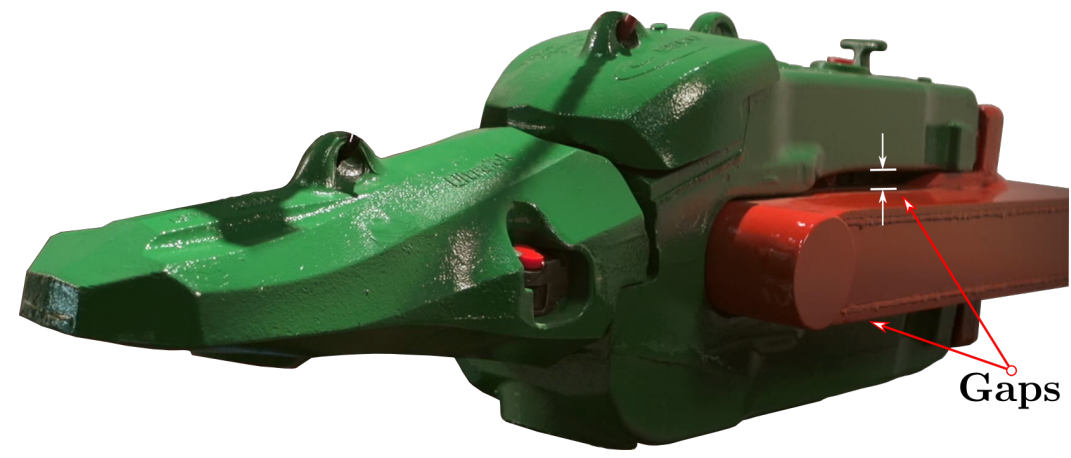

Figure 3.15: The shovel adapter and the gap exploited for wireless communication.

the wireless link budget was enough for a satisfactory connection.

The LoRa ${ }^{\mathrm{TM}}$ modulation uses Chirp Spread Spectrum (CSS) [80]. By configuring the radio parameters, we were able to tune the receiver sensitivity and the data rate. Given our communication requirements (summarized in Figure 3.7) and after some experimenting, we found the values listed in table 3.3 to work well.

We needed to test the wireless link during development. Thus, an actual adapter 
Table 3.3: A summary of the utilized radio parameters.

\begin{tabular}{|c|c|c|}
\hline Parameter & Value & Units \\
\hline Spreading Factor & $7-12$ & \\
\hline Bandwidth & 125 & $\mathrm{kHz}$ \\
\hline Coding Rate & $4 / 5$ & \\
\hline Centre Frequency & $914-916$ & $\mathrm{MHz}$ \\
\hline Preamble & 10 & Symbols \\
\hline Data Payload & $10-255$ & Bytes \\
\hline Transmit Power & 22 & $\mathrm{dBm}$ \\
\hline Receiver Sensitivity & $\geq-140$ & $\mathrm{dBm}$ \\
\hline
\end{tabular}

was needed, which is too heavy to be manipulated without a proper crane. Alternatively, we created a 3D printed mock-up of the adapter's cavity to mimic the electromagnetic effects of the actual one.

\subsubsection{Antenna Matching}

Increasing the battery lifetime of the SN required a closer look at its energy budget. By far, the largest energy consumer on board was found to be the LoRa ${ }^{\mathrm{TM}}$ transceiver. Its hunger for energy is attributed to its integrated front-end power amplifier, which was set to transmit at its maximum output $(22 \mathrm{dBm})$ in order to allow the signal to escape the non-hermetic cavity of the steel adapter. Reducing energy consumption by reducing the transmit power was not favoured because it will directly reduce the link budget and, in turn, the range of the SN and increase the packet loss ratio.

After the preliminary RF experiments, it became clear that a large amount of signal power is lost inside the cavity. Further investigation revealed the significant 


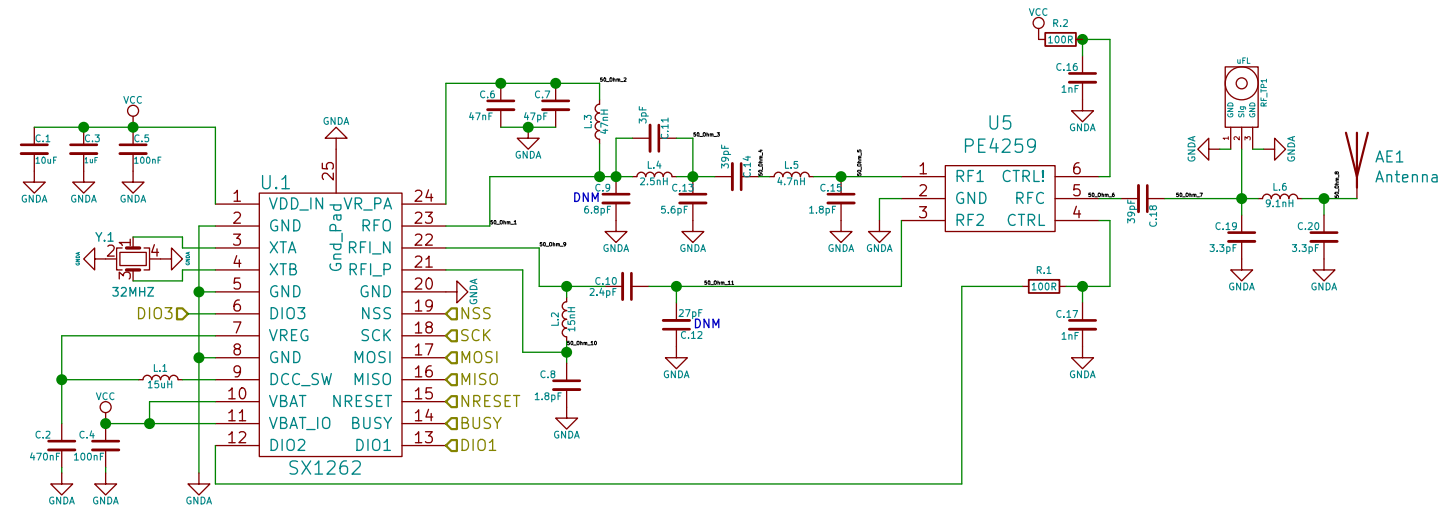

Figure 3.16: The schematic diagram of the LoRa ${ }^{\mathrm{TM}}$ transceiver utilizing the Semtech SX1262 chip.

impedance mismatch between the antenna and the transceiver caused by the shifted impedance of the antenna. The impedance shift is attributed to the presence of the potting silicone rubber and the conductive cavity surface in the antenna's reactive field of the antenna. Thus, we decided to tune the matching network accordingly. With the help of a network analyzer (Rohde \& Schwarz: ZVL 9kHz...3GHz), we performed the following steps in order to match the antenna and increase its radiation efficiency which can increase the link budget enough to allow for a reduction in the transmit power of the transceiver without compromising the link's reliability. Consequently, the battery lifetime is increased, and conflict 1 in Figure 3.7 is eased.

The circuit diagram for the LoRa ${ }^{\mathrm{TM}}$ transceiver is shown in Figure 3.16. This schematic is identical for both revisions however, the layout is different which required similar but independent tuning procedure for each of the two PCB revisions. We started by removing C18 (39 pF) from the assembled PCB to cut the signal path back to the transceiver. Then we connected an RF cable to the RF test point (RF-TP1). Next, the PCB was packaged exactly as it would be when deployed. 


\subsubsection{Cavity Mock-Up}

The mock-up must be identical to the actual cavity from an electromagnetic standpoint. The adapter was reverse engineered by using a laser scanner to generate a point cloud. Then we reverse engineered the point cloud file into a Computer Aided Design (CAD) file format. Finally, we 3D printed an exact replica of the adapter's cavity, as shown in Figure 3.17. One of the major challenges is that the laser scanner provides a mesh model which is different from the solid model needed in CAD tools. There are very few software tools in the market that can convert meshes to solids. In our case, the conversion was made using Fusion 360.

The cavity was printed on a Fused Filament Deposition (FDM) 3D printer using a filament made of polylactic acid (PLA) plastic which is known for its low relative permittivity $\left(\varepsilon_{r}<3\right.$ at $f<3 \mathrm{GHz}$ and $\left.T<200 \mathrm{~K}\right)$. The PLA plastic affects electromagnetic waves in a very different way compared to the original adapter's material (steel). Thus, to mimic the electromagnetic behaviour of the real adapter, the printed cavity had to be covered with an electrically conductive surface whose thickness is sufficiently greater than its skin depth $(\delta)$ at the operating frequency $(915 \mathrm{MHz})$.

We started by covering the surface with a water-based nickel conductive coating (MG chemicals: $841 \mathrm{WB}$ ). Every coat provided a $38 \mu \mathrm{m}$ layer, and by applying the maximum recommended consecutive coats (3), we were able to create a $0.114 \mathrm{~mm}$ thick layer with a surface resistivity of $27 \times 10^{-5} \Omega \mathrm{m}$. Then, we covered the coating with a $48 \mu \mathrm{m}$ thick copper foil with a $40 \mu \mathrm{m}$ thick conductive acrylic adhesive backing (3M: 1/2-6-1126).

The skin depth $\delta$ was calculated to confirm the efficacy of these methods in mimicking the electromagnetic shielding effects of the steel adapter. For most RF bands, 


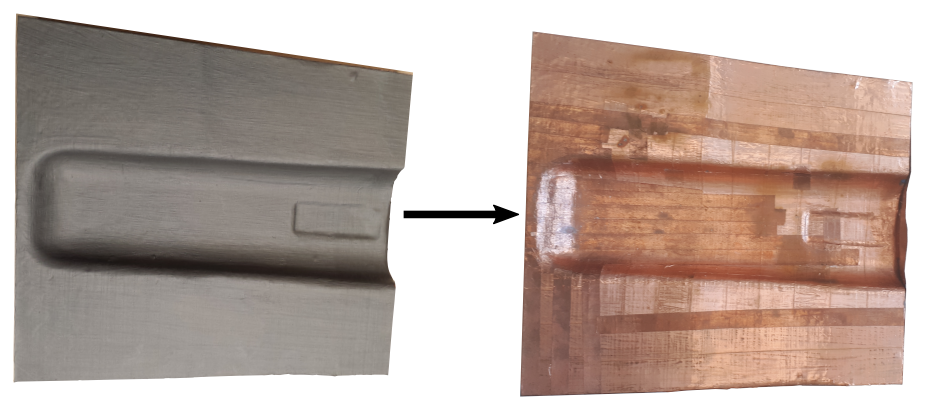

Figure 3.17: The 3D printed cavity mock-up. To the left is after being coated with a conductive coat. To the right is after covering its surface with copper foil.

the skin depth [78] is modelled by equation 3.1 .

$$
\delta=\sqrt{\frac{\rho}{\pi f \mu}}
$$

Where $\rho$ is the resistivity of copper, $\mu$ is the permeability of copper and $f$ is the frequency in $\mathrm{Hz}$. By recalling $\rho_{\text {copper }}=1.68 \times 10^{-8} \Omega \cdot \mathrm{m}$ and $\mu_{\text {copper }}=1.256629 \times 10^{-6}$ $\mathrm{H} / \mathrm{m}$, we calculated $\delta=2.156 \mu \mathrm{m}$ which is significantly smaller than the thickness of just the copper foil, excluding its conductive adhesive backing and the coating. This significant margin accounts for any errors in calculating $\delta$ attributed to using the elemental copper properties instead of the empirical product-specific ratings, which were not made available by the manufacturer of the foil. Thus, we concluded that our lab-created cavity is electromagnetically similar to the steel adapter for RF testing purposes.

Then, as shown in Figure 3.18, we placed the SN inside the cavity mock-up and covered it with a $0.25 \mathrm{~mm}$ thick (sufficiently greater than the skin depth $\delta$ at $915 \mathrm{MHz}$ ) aluminum sheet resembling the bucket's surface when the adapter is attached to it. In order to account for the effect of the RF cable on our impedance measurements, we performed a port extension on the network analyzer (Rohde \& Schwarz: ZVL 


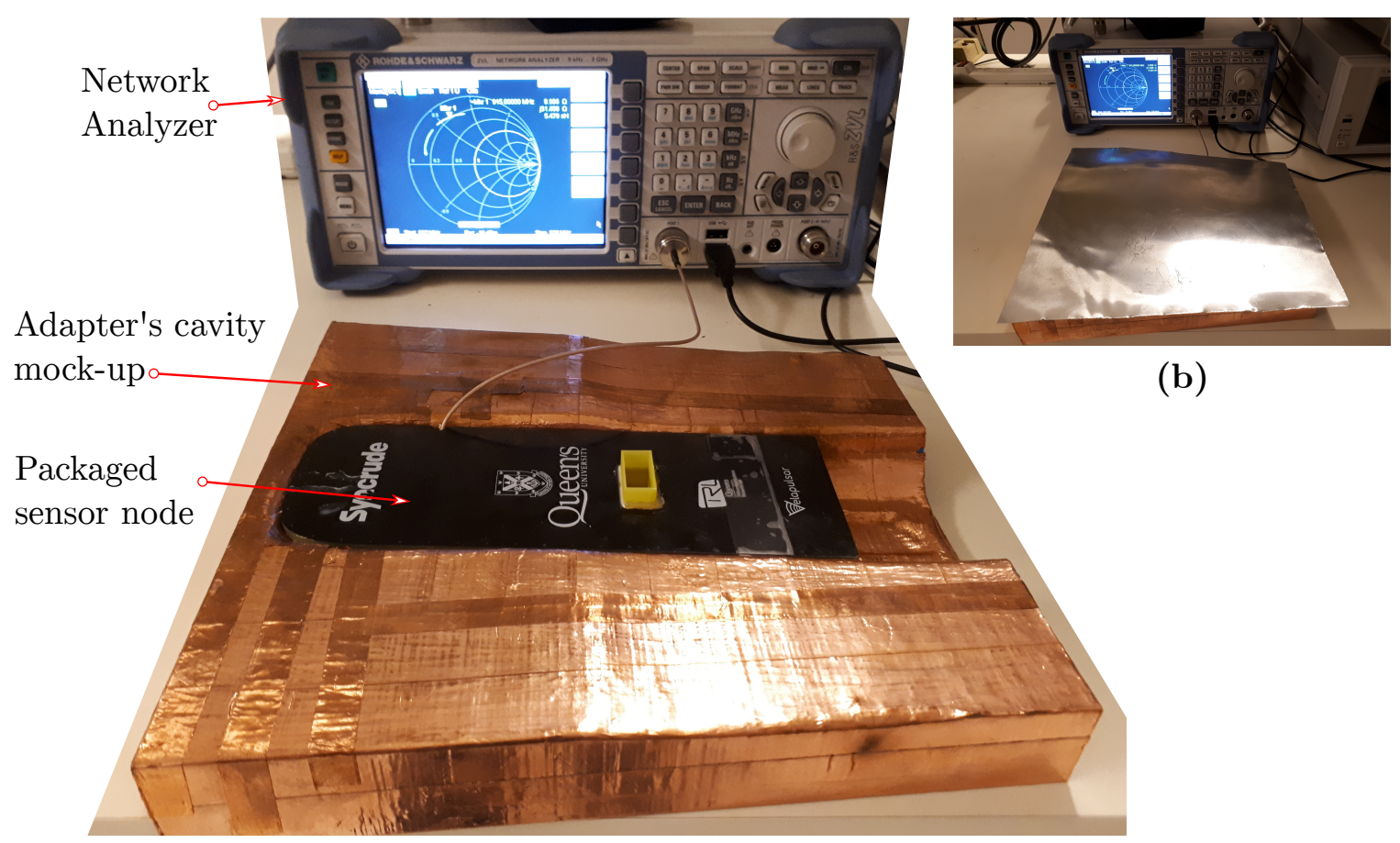

(a)

Figure 3.18: (a) The exposed setup for measuring the input impedance of the antenna inside the final package of the SN. (b) The actual setup when taking measurements after adding the aluminum cover on top.

$9 \mathrm{kHz} \ldots 3 \mathrm{GHz}$ ) using the rated electrical length of our RF cable.

The measured impedance in our setup resembled the impedance of the antenna combined with the impedance of the onboard matching network. Since we already know the values of the $\pi$ matching network components (C19, L6, C20), we were able to solve for the impedance of the antenna alone. Also, we measured the output impedance of the transceiver to capture any deviation from its rated value. This proved to be necessary because the measured value came out as $40-29.5 i \Omega$, which is considerably different from the rated value $(50 \Omega)$. Finally, by using the traditional Smith chart as shown in Figure 3.19 we calculated the new values of the matching network components that will allow for a better impedance matching. 


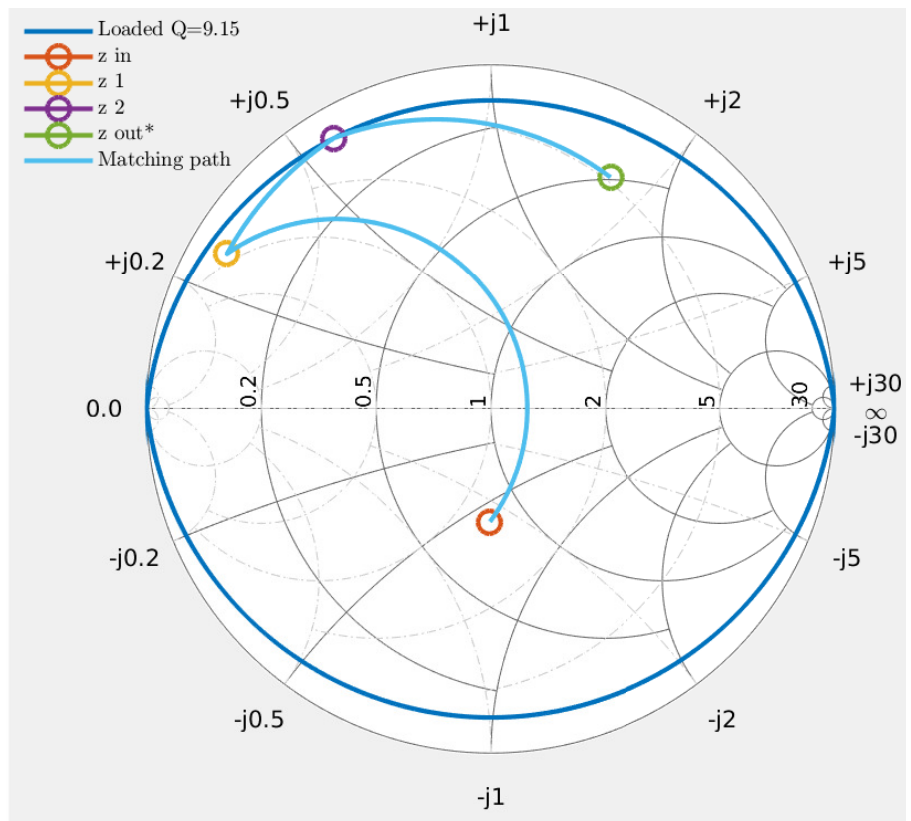

Figure 3.19: The Smith chart used to calculate the matching network components.

The simulated performance of the matching network is shown in Figure 3.20. We can see the reflection coefficient going below -16 dB over our bandwidth $(902 \rightarrow 928$ $\mathrm{MHz})$.

Finally, each PCB was removed from its packaging, all matching network components (C19, L6, C20) were swapped for their new replacements, and C18 was soldered back onboard. Then the tuned PCB was re-packaged and marked. 


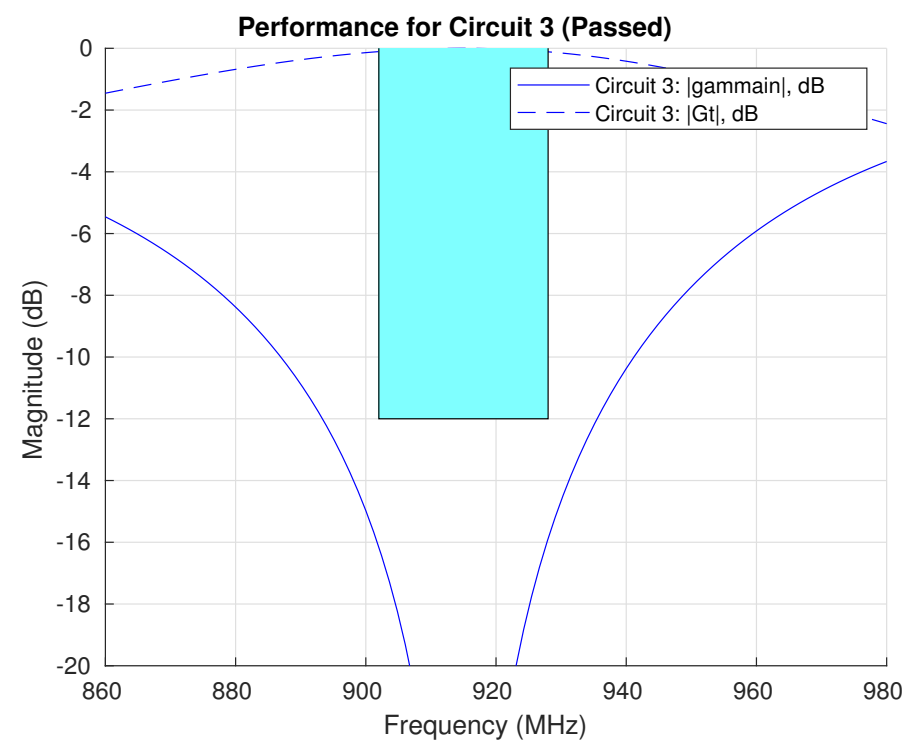

Figure 3.20: The simulated performance of the matching network. |gammain| is the magnitude of the reflection coefficient at the network's input. $|\mathrm{Gt}|$ is the magnitude of the transducer gain.

\subsubsection{MAC Protocol}

The system utilizes a custom-made MAC protocol called MAC On Time (MoT) which allowed for fine-tuning the radio parameters and eliminated unnecessary powerconsuming overhead. The design of this protocol was presented in [1], and an evaluation of its performance was discussed in [75].

The MoT protocol was designed by the lead software developer in our project (Galal Hassan). The synchronous MoT replaced the contention-based LoRaWAN protocol in our system. The primary advantage of the MoT over LoRaWAN is the deterministic latency of each data packet. In other words, every transmitted packet has a deterministic time of arrival that can be controlled and tuned. The deterministic latency is better for real-time applications as opposed to the probabilistic latency of LoRaWAN. 
MoT is centralized, that is, the sink schedules time-slots for the SNs to transmit. Each SN is guaranteed access to the sink within its scheduled time slot.

Compared to LoRaWAN, an SN using MoT consumes less power per packet, and transmit more packets throughout its lifetime independent from the network size. This means, the network scale does not affect the SN's lifetime but affects the latency. For a small number of nodes (less than 50) the latency is suitable for real-time applications.

\subsection{Sensing}

The core sensing function in our SN is fulfilled by a Proximity Sensor (PS), however, other sensors are included to provide data on operating conditions.

The sensing unit collects data from nine different sensors. Five of them are used to sense the proximity of the adapter to the shovel's bucket. The multiplicity of PSs provided enough redundancy to reliably measure the most important monitored variable. Also, the types of PSs were diverse in their sensing mechanism. This allowed for field testing several types and configurations of sensors at the same time.

Sensors $\{1,3,4\}$ are used to probe the proximity of the shovel's bucket. With the use of a very strong magnet in sensor 4, it was a concern that during a detachment event, the glue holding the SN to the adapter's cavity fails, and the magnet latches the SN to the bucket, separating it from the adapter but not the bucket. In this scenario, the readings will fail to indicate any detachment. Therefore, we added sensor 2 to probe the proximity of the SN to the bottom of the adapter's cavity and confirm if the $\mathrm{SN}$ is attached to the adapter at any given time.

We discuss a classification of proximity sensors based on the physical phenomenon 
exploited; this is followed by a discussion of the design challenges of sensors in IIoT applications. Then we will proceed to discuss the design of each sensor/transducer onboard our wireless SN.

\section{Classification of Proximity Sensors}

PSs can be classified according to the physical phenomenon they exploit to sense their targets. EM waves, sound waves, electric fields, and magnetic fields are the four types of physical phenomena governing the operation of PSs as shown in Figure 3.21.

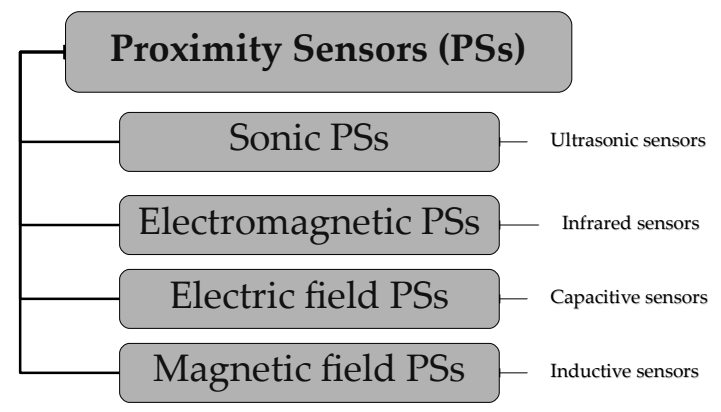

Figure 3.21: Classification of proximity sensors based on the physical phenomenon invoked in sensing the proximity of their targets. An example is noted next to each PS class.

Ultrasonic sensors [81] are a clear example of sonic PSs. In this class, sensors are passive and targets are made of sound reflecting materials (almost all solid materials). Underwater sonars work the same way but, they are not considered PSs because, much like electromagnetic radars, their targets are not in their close vicinity.

The class of PSs using EM waves is very common. In this class, proximity sensing depends on one of two methods. In the first method, the sensor measures and analyses an EM wave reflecting off of the target's surface to extract information about its proximity. Photoelectric sensors [82], gesture recognition radar [83], and image sensors [84] are all good examples of such a method. While aviation radars use the 
same concept, they are not considered PSs because, their targets are not in their close vicinity. In the second less common method, the sensor radiates an EM wave and measures one of the radiation parameters associated with the proximity of its target. Antenna backscattering is an example of this method. The authors in [85] used an RFID tag to sense the proximity of a target. This was achieved by indirectly measuring the impedance mismatch between the tag antenna and the integrated circuit, which is caused by the presence of the target in the close vicinity of the tag antenna.

In the class of electric field PSs, the sensor creates an electric field that gets perturbed by the target causing a shift in the sensor's capacitance. Capacitive sensors [86] are a clear example of this class. This class is very popular with different applications in various domains, such as object identification in robotics [87].

Finally, magnetic field PSs can sense two types of targets based on their magnetic permeability $\mu$. Targets with high permeability can be either active or passive; however, targets with low permeability can only be actively sensed because they have a very weak interaction with magnetic fields. Such targets (low $\mu$ ) are activated by retrofitting them with a permanent magnet, and then a Hall-effect sensor or a reed switch on the sensor side can be used to detect the presence of the retrofitted magnet.

Sensing high permeability passive targets is currently done by one of two methods. The first method is called Eddy-current proximity sensing [88] and, is significantly more popular. It uses a coil to generate a magnetic field that is perturbed when the target is in close vicinity of the coil. This causes a change in the coil's inductance which is then measured by a resonant circuit. This method is utilized by what is commonly known as inductive sensors [89]. The second method is called DC magnetic 
sensing, which uses a permanent magnet as the source of the field. The (MAGNASPHERE: MGB280L) is an example of this method. It uses a magnetized sphere inside a metal casing, as shown in Figure 3.22 . When the target is within range $(\approx 3 \mathrm{~mm})$, the sphere is attracted to the target and moves to the tip of the metal case creating an electrical connection between the centre pin and the case.

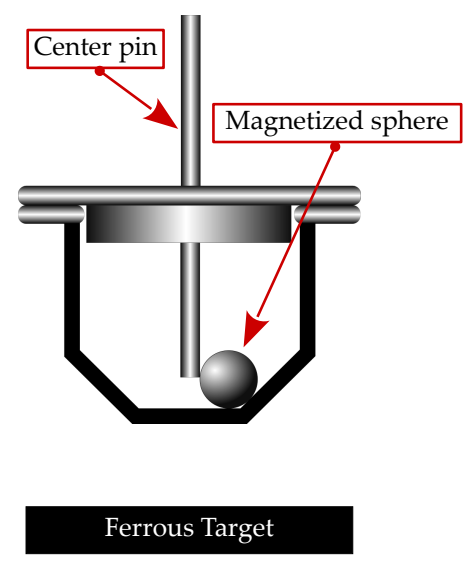

Figure 3.22: The MAGNASPHERE ferrous-selective PSs (MAGNASPHERE: MGB280L).

\subsubsection{Ferrous-Selective Proximity Sensing}

Wireless sensing platforms in IIoT applications have unique challenges. In this section, we will discuss the major challenges related to PSs.

When monitoring construction equipment (e.g., excavators, bulldozers, cranes) in industrial facilities, it is important to monitor vulnerable steel parts that are exposed to extreme mechanical loads. Ferrous-selective PSs narrows down the sensing capabilities to ferrous metals (i.e., steel). This minimizes false measurements in an environment full of uncertainties and various types of debris.

Wired ferrous-selective PSs introduce a significant challenge by placing delicate 
electrical wires in an inhospitable harsh environment. Wireless sensing can circumvent this challenge but has its challenges, such as the battery lifetime, which is a major one. For WSN to make economic sense, the battery life of their SNs has to match or exceed the expected service life of the part being monitored. Also, their cost has to be justified compared to the operational risks they mitigate.

The packaging of an $\mathrm{SN}$ in IIoT applications serves two primary purposes. The first is to protect the sensor from all damaging mechanical loads and/or corrosive chemical compounds. With various packaging requirements for different applications, it is preferred to have a sensor whose package can be easily reconfigured as needed. The second purpose is to allow for the EM waves to pass with minimal attenuation. This limits the suitable materials to only those with low relative permittivity $\left(\varepsilon_{r}\right)$. Composites such as fibreglass, and Kevlar are found to be the best candidates. Although carbon fibre is more mechanically robust, its high relative permittivity [90] disqualifies it.

Metallic surfaces reflect EM waves. With industrial facilities populated with numerous steel structures and equipment, SNs will face a communication challenge. If the $\mathrm{SN}$ is deployed inside a metallic enclosure, significant attenuation to its signal is expected. For a wireless standard based on spread spectrum like the low-power longrange wide area network standard $\left(\mathrm{LoRa}^{\mathrm{TM}}\right)$, the signal can still escape non-hermetic metallic enclosures, as shown in [34]. Another way of tackling the communication challenge is by tuning the WS antenna to the metallic structure in its close vicinity. In other words, matching the output impedance of the transceiver to the impedance of the antenna when it is placed inside the non-hermetic metallic enclosure.

Finally, security systems for buildings and industrial facilities rely on magnetic 
field PSs (i.e., reed switches) for access control and tamper detection. A common attack for security systems utilizing a reed switch is the magnetic activation (MA) attack. In this type of attack, the intruder brings a permanent magnet close enough to the reed switch to keep it in the on-state as its target magnet is moved away. This allows the intruder to gain access without triggering the alarm. Inductive and magnetic force PSs are not prone to this type of attack because their output is a function of the target's material and precise proximity. This means that they can be calibrated on-site for their own targets. Thus, it becomes extremely hard for an intruder to replicate the exact output of these sensors using a foreign object.

Our designed magnetic-force proximity sensor is shown in Figure 3.23. A strong neodymium magnet induces attraction forces between itself and its ferrous target. The Force Sensing Resistor (FSR) is then used to measure the attraction force. The FSR's capacitance is measured instead of its resistance. This is known to improve its accuracy and precision [91]. After calibration for a certain target, a monotonic empirical model can be obtained to express the target's proximity in terms of the FSR's capacitance. 


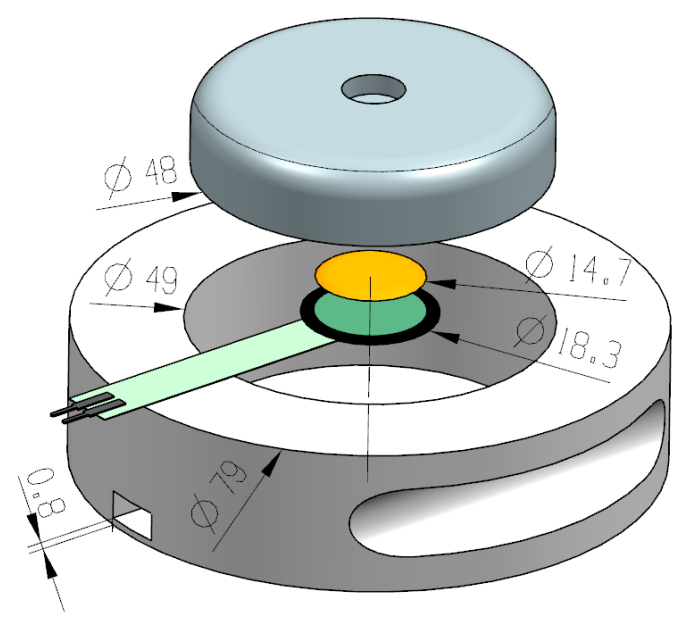

(a)

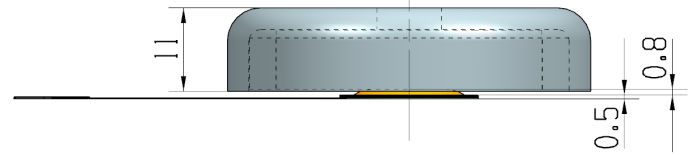

(b)

Figure 3.23: Sensor design. (a) An isometric exploded view. (b) An orthogonal side view showing the magnet on top, the FSR at the bottom and the epoxy layer between them. Dimensions are in $\mathrm{mm}$.

The epoxy layer on top of the FSR has a diameter equal to that of the FSR's active area. This allows the FSR to capture as much as possible of the force exerted by the magnet. While it took numerous trials in the lab to achieve this layer's desired thickness, it is expected to be much easier in an automated manufacturing environment.

Since the proximity of the ferrous target is directly related to the attraction force, it is discouraged to have any ferrous object in the vicinity of thePSsother than the target itself. This will guarantee that the attraction force between the magnet and the target is the only force being measured by the FSR. Thus, there should be a ferrous-free zone around the back of the sensor. The volume of such zone depends on the magnetic field strength of the magnet at its back side. The optimum magnet for this purpose will have a maximum field strength on the side facing its target and no 
field on the opposite side. Since such an idealized permanent magnet does not exist, the next best thing is a mounting magnet, which is a regular neodymium cylindrical magnet housed inside a special cup that redirects the magnetic flux from the back side to the front side.

Magnetostatic simulation tools can simulate and predict the magnetic force if we have good models of the magnet's flux density, the separating medium, and the target's geometry and material composition. Such requirements make simulations only reasonable when a given sensor design has very few use cases in a controlled environment with a fixed target. Even then, considerable discrepancies between simulation results and measurements are expected because of manufacturing tolerances in the magnet and the target, and the per component FSR errors [92]. Hence, experimental calibration of the sensor makes more sense for evaluating its performance and characterizing its output in a certain scenario which is the approach followed here.

Also, exploiting the magnetic attraction force to sense the proximity of ferrous metals enables the sensor to function effectively even when there are obstacles between the sensor and its target. As long as these obstacles are non-ferrous, they will not affect the sensor's operation. This is not true for inductive sensors because they are also affected by other non-ferrous metals such as aluminum and copper [89].

While some inductive sensors are engineered to be selective for ferrous metals, their cost and power consumption are significantly high compared to the proposed sensor design. Furthermore, a major advantage of the proposed design over mainstream inductive sensors is that it does not produce any electromagnetic interference. Static magnetic field of the permanent magnet and the FSR are not able to produce propagating electromagnetic waves. This is an important feature given that future 
IIoT applications will predominantly use embedded sensors and wireless platforms. While inductive sensors might require RF shielding and filtering, the proposed design is inherently non-interfering.

The only foreseeable way this design can produce propagating waves is if the permanent magnet was exposed to high-frequency vibrations (greater than $500 \mathrm{~Hz}$ ) [93]. Even then, the propagated wave would have a very low frequency that can be easily filtered out by most standard RF filters included in any transceiver.

Some specifications of the given sensor design can be deduced from the specifications of its off-the-shelf components. This allows developers to create a preliminary evaluation of the sensor's performance without building a functional prototype. For example, the operating temperature range is simply the intersection of all ranges given by the utilized components (FSR, magnet).

The operating proximity range $\left(P_{o p}\right)$ can be estimated in two steps. First, with the help of a Universal Testing Machine (UTM), we develop an empirical model of the magnetic attraction force $(F)$ as a function of the target's proximity $(P)$ as shown in Figure 3.24. Second, the FSR's minimum and maximum operating forces are superimposed on the curve to identify the proximity range of this setup. 


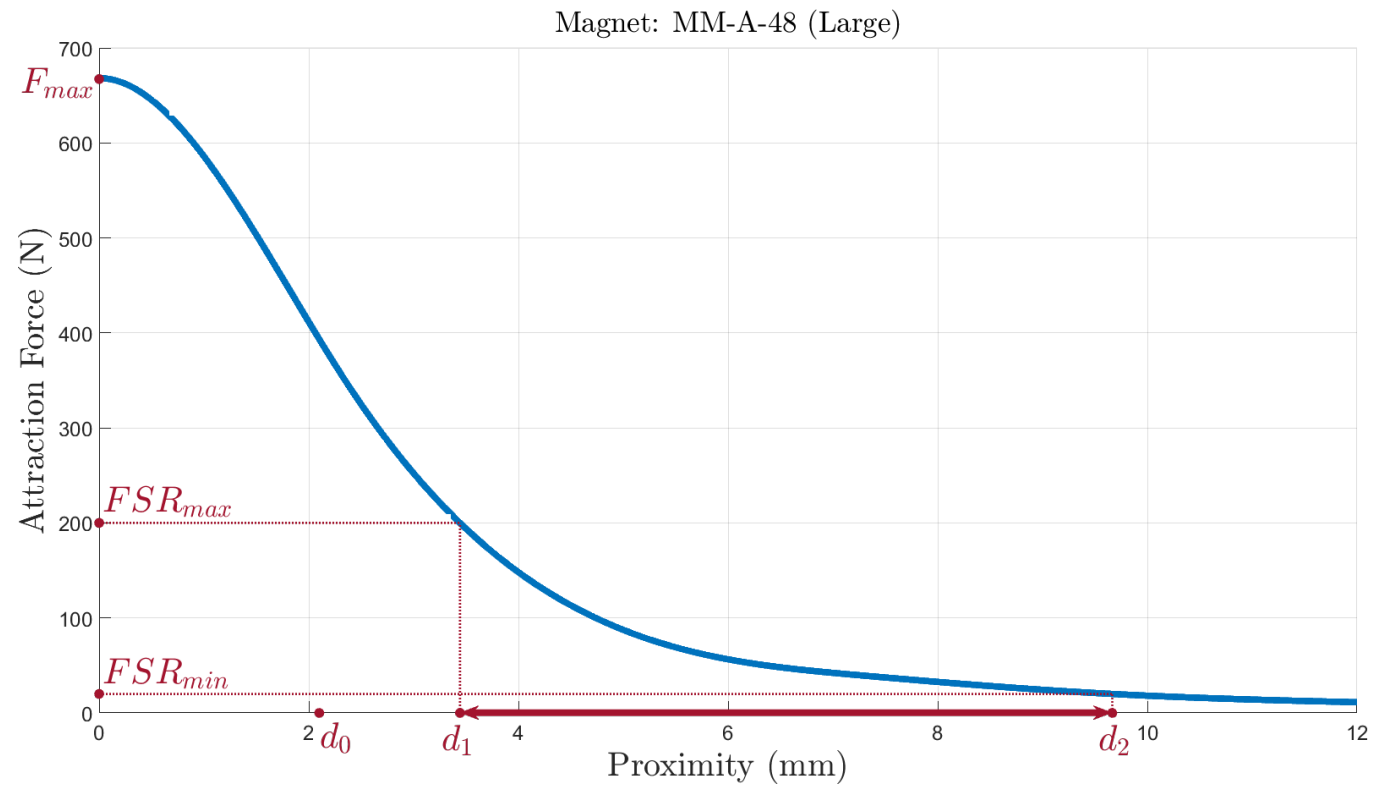

Figure 3.24: Empirical model of the magnetic attraction force as a function of the target's proximity.

If $P(f)$ is the target's proximity at $F=f$, then $P_{o p}$ can be calculated as follows.

$$
P_{o p} \in\left[\max \left(d_{0}, d_{1}\right), d_{2}\right]
$$

such that,

$$
\begin{aligned}
& d_{1}=P\left(\min \left(F S R_{\max }, F_{\text {max }}\right)\right) \\
& d_{2}=P\left(\max \left(F S R_{\text {min }}, W_{\text {mag }}\right)\right)
\end{aligned}
$$

where $P_{o p}$ is the operating proximity range. $F S R_{\max }, F S R_{\min }$ are the maximum and the minimum rated force for the FSR, respectively. $F_{\max }$ is the maximum attraction force induced by the magnet, which is exerted when the target is in complete 
contact with the magnet, and $W_{m a g}$ is the magnet's weight, $d_{0}$ is the distance between the magnet's face and the outer face of the sensor's enclosure. For the design shown in Figure $3.23, d_{0}$ is the total sum of the thicknesses of the epoxy layer $(0.8 \mathrm{~mm})$, the FSR $(0.5 \mathrm{~mm})$, and the bottom surface of the enclosure $(0.8 \mathrm{~mm})$ as shown in Figure $3.23(\mathrm{a})$.

The proposed design can be easily tuned to satisfy different application requirements by changing the magnet and/or the force sensing device. Also, its low-power consumption allows it to be battery powered and embedded in a hermetically sealed wireless module for aerospace, mining, or any other harsh environment application.

One of the commonly used sensors in almost every application domain is the proximity sensor (PS). APSscan be defined as a device used to sense the presence of a physical object (often called a target) in its spatial vicinity. This definition establishes a distinction between PSs and range sensors. While range sensors rely on the same concepts, their targets are not in their spatial vicinity.

Applications of PSs are spread across a wide range of industries. A few examples are: manufacturing, mining, transportation, building automation, and consumer electronics. Their widespread use is rooted in their ability to satisfy common application requirements in motion control, safety [94], access control [95], fault detection [96], interactivity [97], and quality control [?]. As we move towards more automation, the utilization of sensors in general and PSs, in particular is only expected to grow.

Proximity sensors sense either active or passive targets. A passive target conveys its presence to the sensor by perturbing a field or interacting with a propagating wave created by the PS. On the contrary, an active target will create such fields and/or waves itself, and the sensor is then used to probe them. Active targets have 
to be modified or retrofitted with a piece of hardware to make them detectable to their sensors, while passive targets do not require any modifications. In industrial applications, passive sensing is preferred because retrofitting the target with magnets or any other piece of hardware is sometimes not desired or even infeasible. Also, passive sensing reduces the installation overhead incurred by active sensing.

Ferrous-selective PSs are currently available [89]; however, their power consumption is too high (typically hundreds of $\mathrm{mW}$ ) for battery-powered WSs. There is a current need for low-power passive proximity sensors that are selective to ferrous metals (e.g., steel). The only low-power option available commercially is just a detector, not a sensor (i.e., its feedback is binary). Also, it has a relatively small maximum range of $4.29 \mathrm{~mm}$ (MAGNASPHERE: MGB280L).

Ferrous-selective PSs can be useful for monitoring the integrity of steel structures and automating their assembly, monitoring construction equipment, hermetic vessels such as submarines, mining equipment, and aerospace vehicles. They can also be used in process control applications to monitor ferrous-metal machine parts or products on a production line.

We propose a low-power, low-cost, ferrous-selective, passive proximity sensor with a maximum reported range of $20 \mathrm{~mm}$ and the option to extend it even more by tweaking the design parameters. The proposed sensor uses a permanent magnet and a FSR to provide a configurable dynamic range with good resolution while consuming significantly less power $(\approx 8 \mathrm{~mW})$ [98]. Results indicate very good sensing capabilities with flexible design parameters that can satisfy various requirements. 


\section{Experimental results}

In this section, we will discuss the experimental work for the proposed sensor and present an evaluation of its performance using different magnets.

The presented experiment is a design instance of the proposed sensor. The authors believe that other instances with a different layout and/or parameters can be more suitable for other applications.

Setup A UTM (ZwickRoell Z020) equipped with an accurate load cell $( \pm 0.1 \mathrm{~N})$ was used to benchmark the force measurements collected from the FSR. The speed of the UTMs during all the runs was $10 \mathrm{~mm} / \mathrm{min}$ with a step resolution of $0.1 \mathrm{~mm}$. The non-ferrous enclosure was 3D-printed with a fused filament fabrication (FFF) 3D-printer using polylactic acid (PLA) filament. The FSR rested on a $(0.8 \mathrm{~mm})$ layer of PLA plastic, and its active area was coated with a thin layer $(0.8 \mathrm{~mm})$ of epoxy to improve precision [99]. The epoxy layer is the orange-coloured layer shown in Figure 3.23 .

A plastic holder was used to keep the magnet far from the UTMs's top clamp (steel) to eliminate any attraction forces between the magnet and the top clamp. A thick $(\approx 25 \mathrm{~mm})$ carbon steel target was placed on the bottom. The sensor was positioned such that its bottom surface is parallel to the target's surface.

In order to quantify the FSR's hysteresis [100], the test was performed in compression mode (approaching) as well as in tension mode (receding). Using a Capacitance Digital Converter (CDC) (Texas Instruments: FDC2214), the FSR's capacitance was measured instead of its resistance. This was shown to produce more accurate measurements and improve precision [91]. 


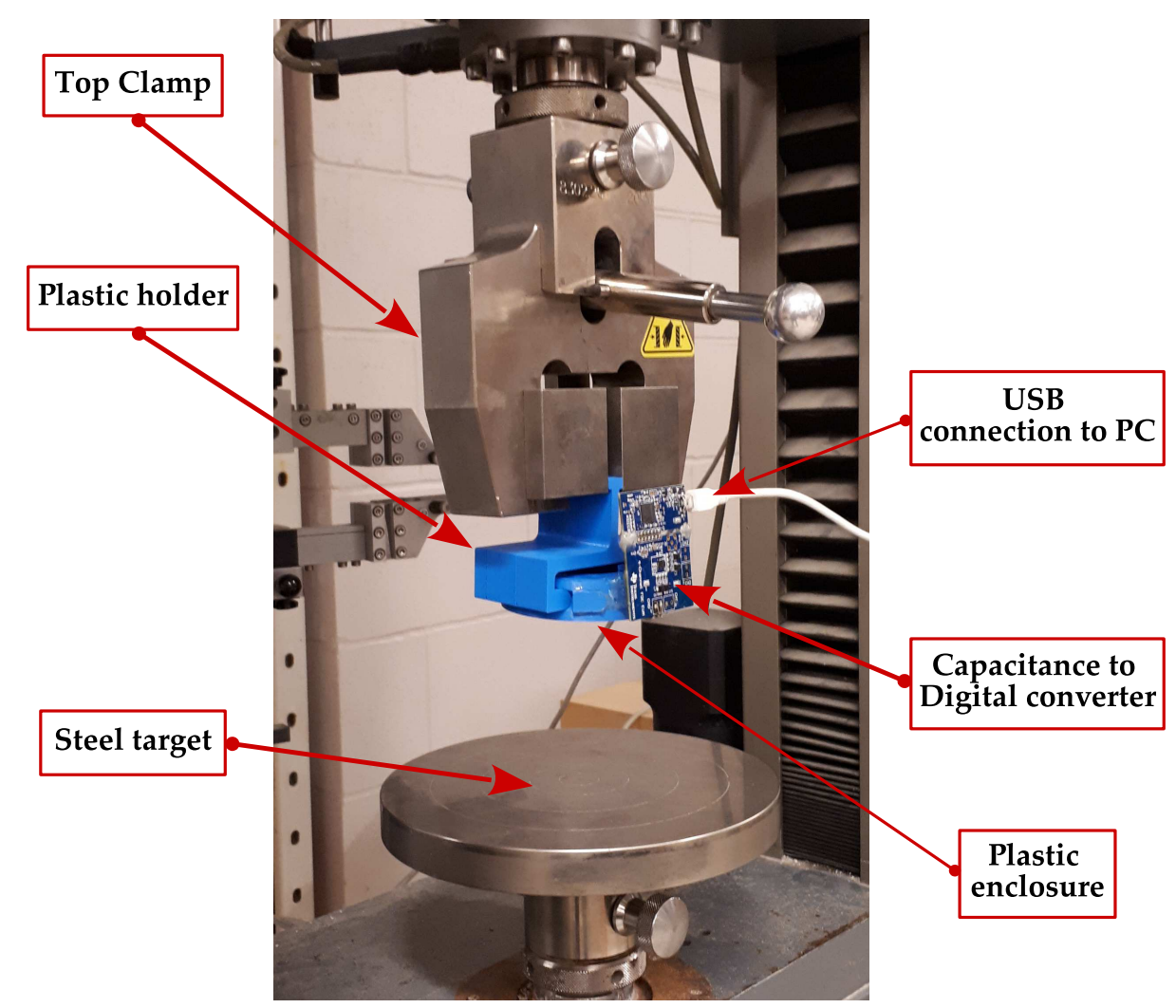

Figure 3.25: The experimental setup on the UTM.

Three different disc-shaped neodymium magnets of the same grade (N38) were tested to study the effect of the magnet size on the operating proximity range $P_{o p}$. These magnets were marketed as mounting magnets; their magnetic field has a lot more strength on one side than the other. This helps in reducing the size of the ferrous-free zone at the back side of the sensor. The FSR and the magnets' diameters are listed in Table 3.4.

Results and Discussion The results show good potential for the proposed sensor design. The measurements were post-processed to compensate for the effect of the sensor's weight. 
Table 3.4: Specifications of components used in the experiment.

\begin{tabular}{|c|c|c|c|}
\hline Component & Manufacturer & part number & Diameter \\
\hline Large magnet & K\&J Magnetics Inc. & MM-A-48 & $48 \mathrm{~mm}$ \\
\hline Medium magnet & K\&J Magnetics Inc. & MM-A-36 & $36 \mathrm{~mm}$ \\
\hline Small magnet & K\&J Magnetics Inc. & MM-A-20 & $20 \mathrm{~mm}$ \\
\hline FSR & Interlink Electronics & 402 & $14.7 \mathrm{~mm}^{*}$ \\
\hline CDC & Texas Instruments & FDC2214 & N/A \\
\hline
\end{tabular}

* diameter of the active area only

The fundamental laws of magnetism predict the maximum attraction force to be at the smallest separation distance (i.e., zero). However, as shown in Figures 3.26, 3.27 , and 3.28 , the measured force is not at its maximum when the proximity is zero. We notice the force, as measured by the UTM, starts from zero when the target is very far and increases exponentially as it approaches the sensor until it reaches a maximum value at a few millimetres away from the target, then it decreases rapidly till it goes back to zero when the target is in contact with the sensor. This behaviour occurred in both, the approaching and the receding runs.

Careful observation of the experiment's fixtures revealed the reason for this behaviour. As the top clamp of the UTMs approaches the bottom target, the attraction force increases and the 3-D printed plastic enclosure experiences significant loading forces. Consequently, the structure flexes and its bottom surface buckles towards the target. As a result, the sensor's enclosure comes into contact with the target before the UTMs reaches its calibrated zero position. Once the enclosure is prematurely in contact with the target, the magnetic attraction force starts to be off-loaded to the 
target, which reduces the amount of force exerted on the UTMs's load cell and the FSR. As the proximity gets closer, more force is off-loaded to the target and even less is applied on the FSR. This behaviour continues until the UTMs reaches its calibrated zero position, at which there is no buckling in the enclosure. At this point, the load cell of the UTMs measures zero while the FSR is still squeezed between the magnet and the target. That is why the capacitance does not follow the force all the way down to zero. This effect is severe with the large magnet and diminishes with smaller magnets, as shown in Figure 3.29. This is because the distance at which it starts to occur gets smaller as the magnet's size is reduced. A stiffer material for the enclosure than PLA will help minimize this effect when large magnets are used.

As shown in Figure 3.26, the capacitance measurements for the large magnet show an exponential decay similar to that of the force. However, midway through the experiment, it violates the trend with a semi-flat region and then resumes the exponential decay afterwards. A reduced version of this highly non-linear behaviour is noticed in the medium magnet as in Figure 3.27 and no such behaviour is noticed in the small magnet as in Figure 3.28.

Two reasons have contributed to this behaviour. The first reason is that the FSR has operated beyond its rated maximum force of $20 \mathrm{~N}$. The small magnet is an exception which is why it does not show the same non-linear behaviour. On the other hand, the capacitance of the FSR is monotonically decreasing with proximity. Thus, a single-valued function can be obtained to relate the proximity to the capacitance. This highlights the importance of configuring the sensor design for each application with the appropriate magnets and FSRs to obtain a relatively linear output over its operating range. 
The second reason lies with the epoxy resin dome on top of the FSR. While it was reported to improve the FSR's precision [99], in this case, its effectiveness depends on the ratio $\left(R_{d}\right)$ between the magnet's diameter and the diameter of the FSR's active area. Since the resin dome is not perfectly flat, the magnet on top of it can tip slightly, causing it to rest also on the enclosure's bottom surface, not just on the FSR. This behaviour is more likely to occur when the magnet's diameter is bigger than the diameter of the FSR's active area.

In the case of the large magnet in Figure $3.26, R_{d}$ is 3.28 , and the flat region of the curve occupies a large portion of the exponentially decaying part of the curve. As shown in Figure 3.27, when $R_{d}$ got reduced to 2.4 with the medium magnet, the flat region occupied a smaller portion of the curve. Finally, when $R_{d}$ was further reduced to 1.37 with the small magnet as in Figure 3.28, the flat region did not show up at all in the measurements.

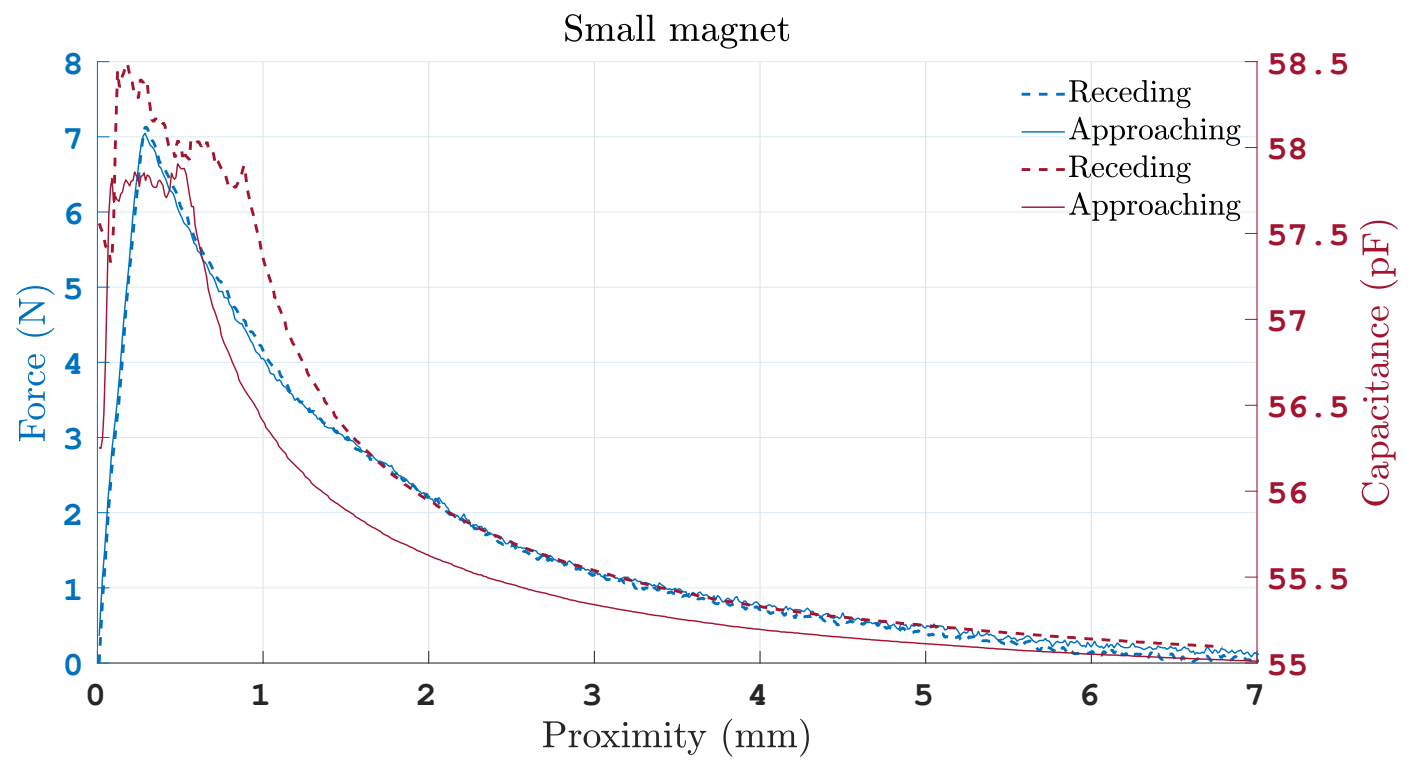

Figure 3.26: Target's proximity vs. attraction force (left) and the FSR's capacitance (right) using the large magnet. 


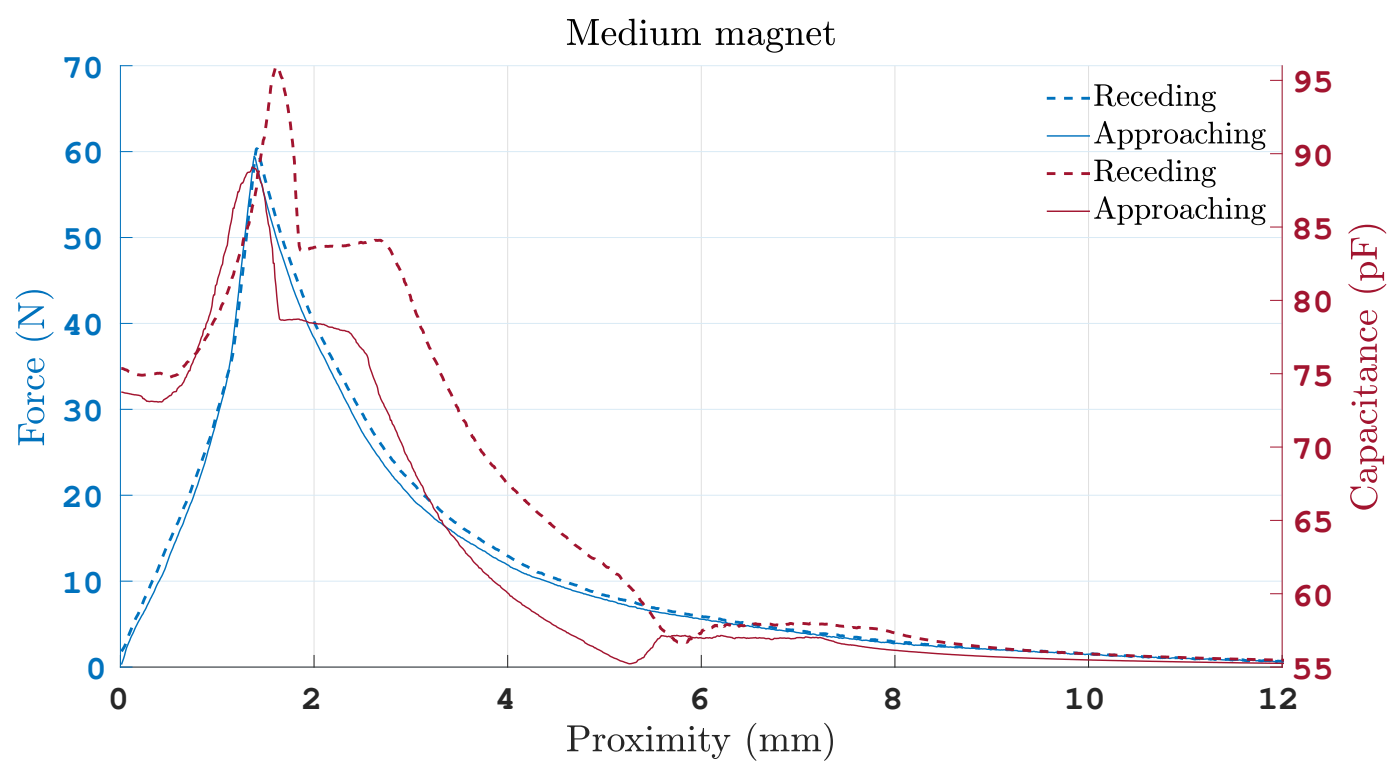

Figure 3.27: Target's proximity vs. attraction force (left) and the FSR's capacitance (right) using the medium magnet.

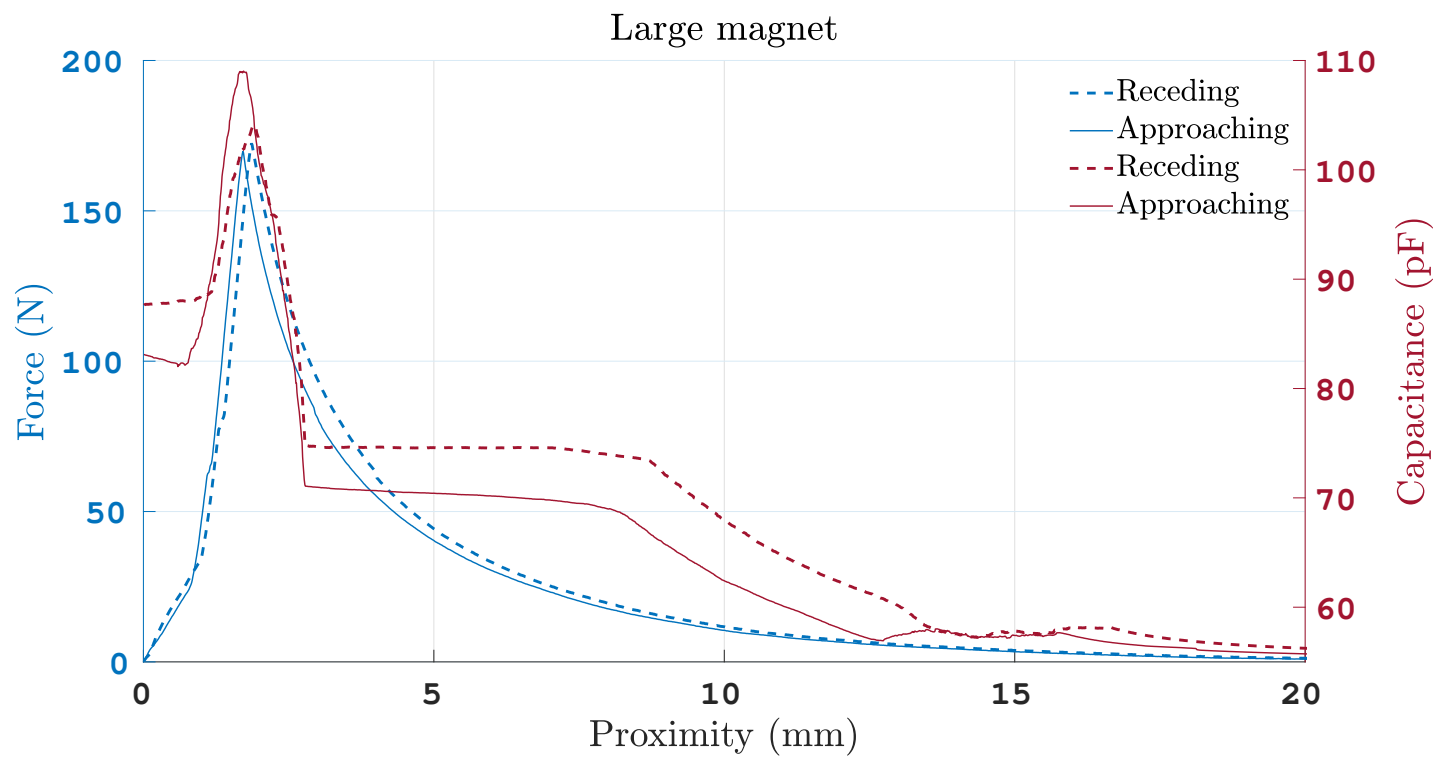

Figure 3.28: Target's proximity vs. attraction force (left) and the FSR's capacitance (right) using the small magnet. 


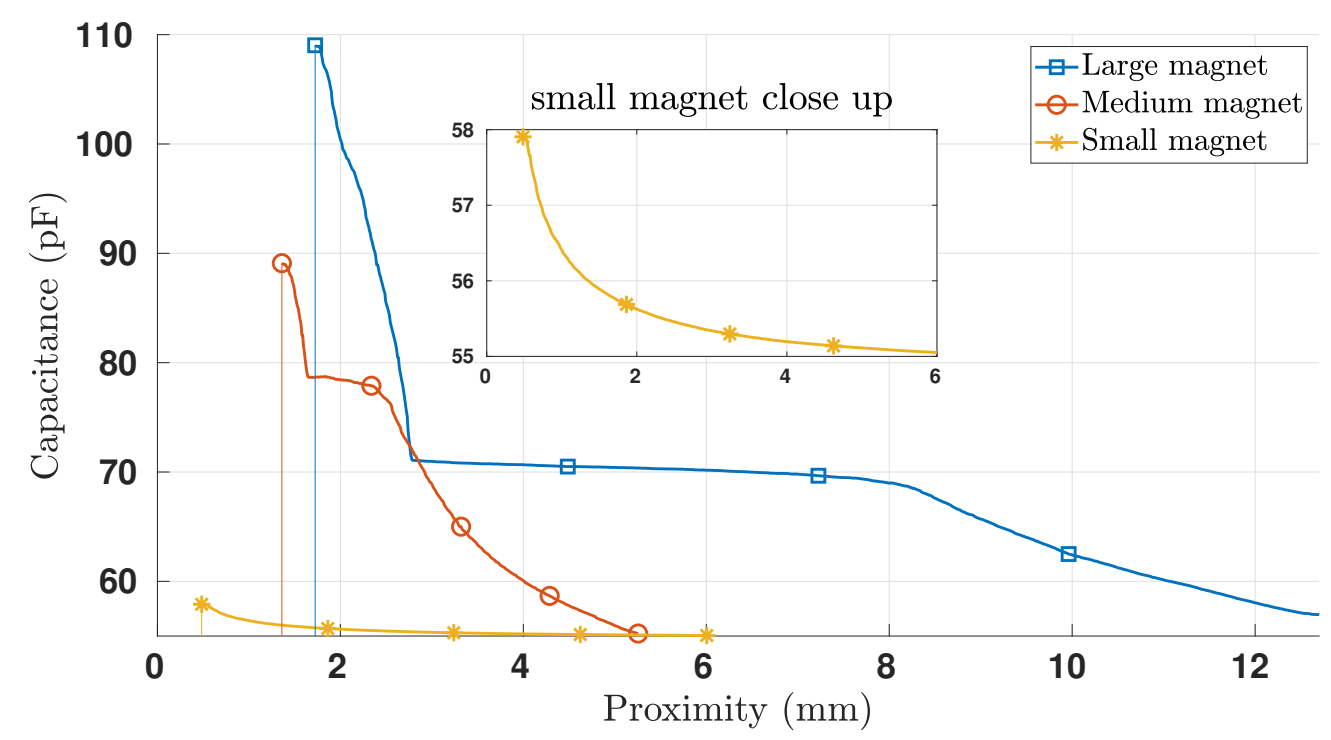

Figure 3.29: Output comparison of the three magnets.

In Figure 3.29, the small magnet appears to give a relatively small variation in capacitance $(\approx 3.5 \mathrm{pF})$ which can invoke a concern over the resolution of the PS; however, the utilized CDC [98] has a noise floor of $0.3 \mathrm{fF}$ which enables it to quantize the given capacitance range into 11666 levels. This is more than sufficient for providing adequate resolution over the small change in capacitance. Thus the performance limiting factor here remains the accuracy and precision of the FSR, not the CDC. Also, the high sampling rate of the chosen CDC (4.08 ksps) enables the sensor to reliably sense dynamic targets with a response time dictated by the FSR's rise time $(<3 \mu \mathrm{s})$. While for static targets, the long-term drift of the FSR $(<5 \%$ at $1 \mathrm{~kg}$ load for 35 days) is of more concern.

Applying excessive force on the FSR will cause plastic deformation and damage the sensor. Our chosen FSR had a plastic deformation pressure of $10 \mathrm{~N}$ per $\mathrm{mm}^{2}$ of active area. From table 3.4 , the active area equals $\pi\left(\frac{14.7}{2}\right)^{2} \approx 170 \mathrm{~mm}^{2}$. Thus, the 
deforming force is $\geq 1.7 \mathrm{kN}$ which is much larger than the loading conditions in our experiment where the highest force measured is $\approx 0.175 \mathrm{kN}$.

\subsubsection{Capacitive Proximity Sensor (Bucket Side)}

for many applications [101]. In this case, it monitored the proximity of the bucket's surface to the adapter's cavity. The self-capacitance $\left(C_{s}\right)$ of a large circular copper pad changes in response to the proximity of any conductive surface. The pad is connected to a resonant circuit and a CDC measures the resonant frequency and use it to calculate $C_{s}$. The CDC used a temperature compensated oscillator (Microchip Technology: DSC6013CI2A-033.0000) in order to minimize frequency shifts due to temperature changes during operation.

The electrode's geometry determines many of the performance metrics such as

the sensing range, sensitivity, and dynamic range [101]. We used a single-electrode circular capacitor with the largest possible diameter $(50 \mathrm{~mm})$ to maximize the range and achieve the most uniform sensitivity distribution, as noted in [102]. Furthermore, the sensing pad was printed on a four-layer PCB stack which allowed for including a passive ground shield right underneath the pad. The shield helps in focusing the electric field on the target (bucket's surface).

The capacitive sensors use a resonant circuit at their front end, whose resonant frequency is the basis of their measurements. Thus, in order to avoid any interference between onboard components, we included a passive RF filtering circuit at each input of the CDC. 


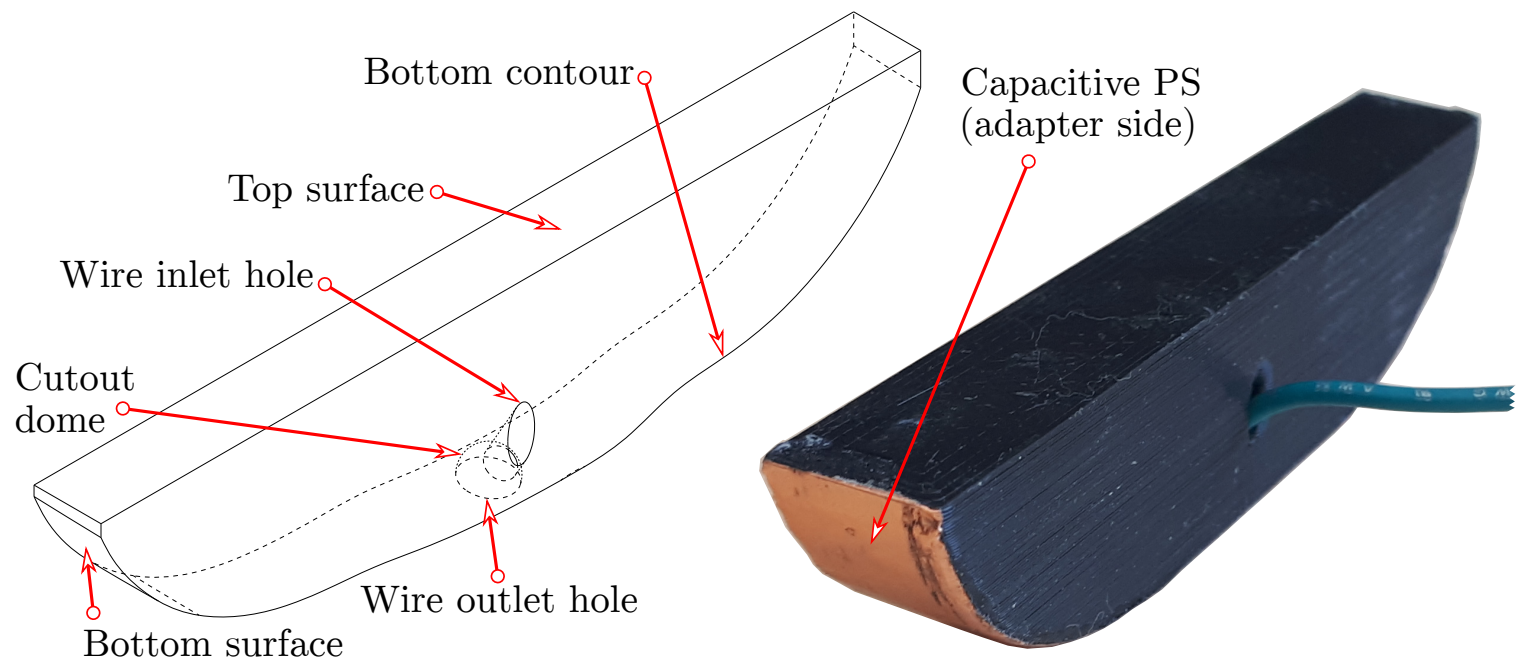

(a)

(b)

Figure 3.30: The Polycarbonate (PC) support at the end of the SN package.

\subsubsection{Capacitive Proximity Sensor (Adapter Side)}

A second capacitive PSs monitors the proximity of the SN to the adapter's cavity as shown in Figure 3.30. This sensor utilized a piece of copper foil attached to the adapter's side of the SN. The foil is connected back to the PCB through the green wire as shown in Figure 3.30. The wire was soldered to the back side of the foil (the solder blob was housed inside the cutout dome) and routed through a designed internal pathway in the Polycarbonate (PC) support which was 3D printed in a closed chamber Fused Deposition Modeling (FDM) printer with a high-temperature nozzle.

\subsubsection{Inductive Proximity Sensor}

The inductance $(L)$ of a circular PCB coil changes in response to the proximity of any conductive surface. The PCB coil is again connected to a resonant circuit where an Inductance Digital Converter (IDC) (Texas Instruments: LDC1614) is used to 
measure the resonant frequency and then calculate $L$. Our design increased sensing range while keeping the power consumption low by using a PCB coil made of four layers with an outer diameter of $60 \mathrm{~mm}$ and 60 turns per layer, yielding a total inductance of $L=2.22 \mathrm{mH}$ and a power dissipation of $6 \mu \mathrm{W}$ (for the coil only).

Similar to the CDC, the IDC also had a passive RF filter at its front end to reduce interference levels in its resonant circuit.

\subsubsection{Wear-Level Sensor}

As shown in Figure 3.31, the sensor is a thin Printed Circuit Board (PCB) probe that fits inside a small hole in the wear part of the tooth. As the tooth wears out, this probe wears out too and provides accurate thickness measurements, which allows for monitoring the wear level of teeth. This sensor is basically a parallel plate capacitor fabricated on a PCB and connected to the onboard CDC. The length of the probe is a function of its capacitance.

It is worth mentioning that the dynamic range of the probe's capacitance can be easily manipulated. For example, it can be fitted to a staircase function by utilizing a multiple plate capacitor with various geometries embedded in a multi-layer PCB stack. 


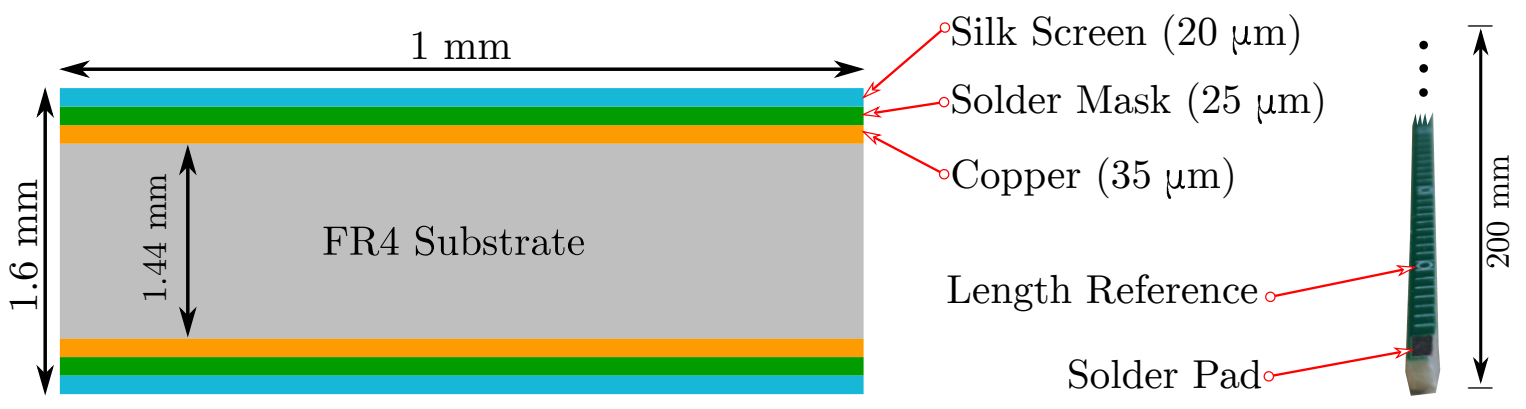

Figure 3.31: Left: a cross section of the wear-level sensing capacitive probe showing its PCB stack-up. Right: a photo showing the first few centimetres of the manufactured probe.

\subsubsection{Light Sensor}

A Light Detecting Resistor (LDR) is used to sense the intensity of ambient light around the SN. When the adapter is intact and fully attached to the bucket, the light entering its internal cavity (where the $\mathrm{SN}$ is installed) is expected to be minimal. On the contrary, when the adapter is broken/detached, the SN will be exposed to more light. Hence, ambient light can indicate the attachment state of the adapter. However, the difference in ambient light between day and night necessitates timestamping of all measurements in order to use the right thresholds.

Specifying the right thresholds is crucial to differentiate between the dark (attached) and the light (detached) states. This threshold is unknown during the design stage. Calibration is needed in the field to identify suitable thresholds. Taking field measurements during the design stage was not an option due to the high cost of lost oil production associated with shovel downtime. Thus, the specification of the thresholds has been handled by the firmware so it can be easily adjusted remotely.

To ensure we can capture the full variation in ambient light, we needed to maximize the dynamic range of the output of the light-sensing circuit shown in Figure 


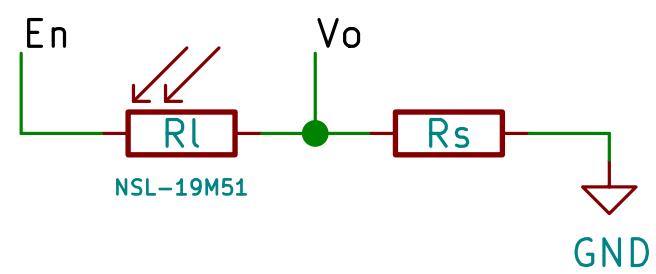

Figure 3.32: The circuit diagram of the ambient light sensor.

3.32. In this simple voltage divider circuit, $R_{l}$ is the LDR, $R_{s}$ is the series resistor, $V_{o}$ is the output voltage which connected to the integrated Analog to Digital Converter $(\mathrm{ADC})$ in the microcontroller, $E_{n}$ is the enable signal coming from one of the digital outputs of the microcontroller to power the circuit only when we are taking a measurement for energy-saving purposes. When the circuit is enabled, $E_{n}=V_{c c}$.

For a simple voltage divider circuit, $V_{o}$ is basically given by equation 3.3

$$
V_{o}=\frac{V_{c c} R_{s}}{R_{s}+R_{l}}
$$

The optimum value of $R_{s}$ is the one that maximizes the dynamic range of $V_{o}$ as this would allow for maximum resolution for our measurement. Consequently, we want to find the formula for the dynamic range of $V_{o}$ and find the value of $R_{s}$ that maximizes it. From equation 3.3 we note that $V_{o} \propto \frac{1}{R_{l}}$, This means that the maximum value for $V_{o}$ (denoted by $V_{o \uparrow}$ ) exists at the minimum value of $R_{l}$ (denoted by $R_{l \downarrow}$ ) and the minimum value for $V_{o}$ (denoted by $V_{o \downarrow}$ ) exists at the maximum value of $R_{l}$ (denoted by $\left.R_{l \uparrow}\right)$. Therefore,

$$
V_{o \uparrow}=\frac{V_{c c} R_{s}}{R_{s}+R_{l \downarrow}} \quad \text { and } \quad V_{o \downarrow}=\frac{V_{c c} R_{s}}{R_{s}+R_{l \uparrow}}
$$

Let $D R$ be the dynamic range of $V_{o}$ where $D R=V_{o \uparrow}-V_{o \downarrow}$ then, to find the value 
of $R_{s}$ that will maximize $D R$, we take the partial derivative $\frac{\partial D R}{\partial R_{s}}=0$ hence,

$$
\frac{18\left(R_{l \uparrow}-R_{l \downarrow}\right)\left(R_{l \uparrow} R_{l \downarrow}-R_{s}^{2}\right)}{5\left(R_{l \uparrow}+R_{s}\right)^{2}\left(R_{l \downarrow}+R_{s}\right)^{2}}=0
$$

to solve equation 3.5, we need to employ some understanding of the physical limits of this problem. By examining the denominator of equation 3.5, we realize it is always positive; therefore, the whole fraction is zero when its numerator is zero. Also, $18\left(R_{l \uparrow}-R_{l \downarrow}\right) \neq 0$ because, $R_{l \uparrow}$ is always greater than $R_{l \downarrow}$ therefore,

$$
R_{l \uparrow} R_{l \downarrow}-R_{s}^{2}=0
$$

Finally, the $R_{s}$ that maximizes $D R$ is given by

$$
R_{s}=\sqrt{R_{l \uparrow} R_{l \downarrow}}
$$

Notice how $R_{s}$ is not a function of $V_{c c}$ which means as the battery's voltage decreases along its discharge curve, we will still get the maximum $D R$ for the current value of $V_{c c}$. Also, equation 3.7 applies to any variable resistance sensor in a voltage divider not just an LDR.

As for our case, the chosen LDR (Advanced Photonix: NSL-19M51) has a rated $R_{l \uparrow}=20 \mathrm{M} \Omega$ and $R_{l \downarrow}=20 \mathrm{k} \Omega$. Thus, by substituting in equation 3.7 we calculate $R_{s}=635 \mathrm{k} \Omega$ 


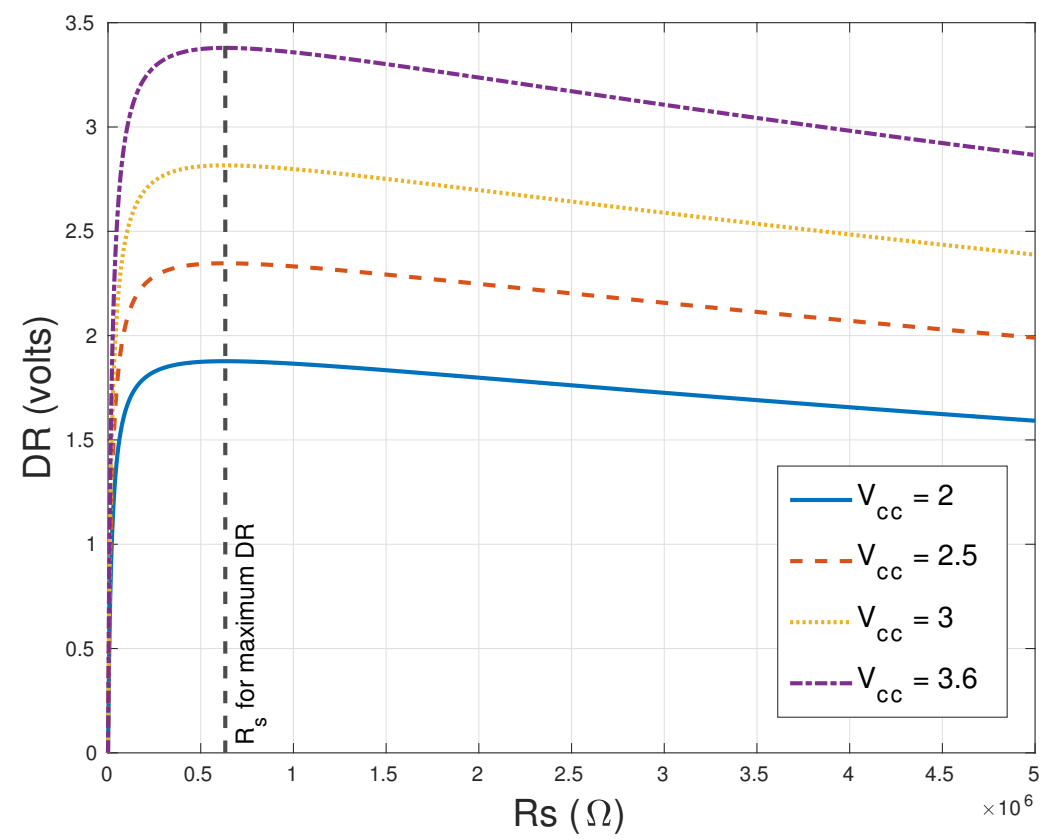

Figure 3.33: The dynamic range $(D R)$ of the light sensor $V_{s}$ the series resistance $\left(R_{s}\right)$. The value of $R_{s}$ that maximizes $D R$ is constant across all values of $V_{c c}$ varied along the typical discharge curve of the onboard battery $(3.6 \rightarrow 2 \mathrm{~V})$.

\subsubsection{Vibration Sensor}

Saving power in order to extend the lifetime of the SN meant that all the chips onboard should be in a deep sleep mode when the shovel is idle. We chose vibration as a proxy for the operational state of the shovel (idle vs active).

A low-power accelerometer (STMicroelectronics: LIS2HH12) is used as a vibration sensor. Whenever the shovel is idle (i.e., no vibrations), the controller shuts down the sensors, the transceiver and enters a deep sleep mode to save energy. Bearing in mind that only an external interrupt signal can wake up the controller from the deep sleep mode, the accelerometer wakes it up when the shovel is active again (i.e., vibrations detected). 
While the accelerometer provided sophisticated ways of sensing vibration as an indication of the shovel's active state, we found out that the significant firmware development overhead makes it less appealing compared to a simple omnidirectional vibration sensor (Sensolute: MVS0409.02), which was used in an earlier revision of our design instead of the accelerometer.

\subsubsection{Temperature Sensor}

The SN's operating temperature was set throughout the design process as $-40^{\circ} \mathrm{C}$ to $85^{\circ} \mathrm{C}$, which was worked out in a previous paper [34]. To support troubleshooting in case of a node failure, the operating temperature is regularly reported using a temperature sensor integrated inside the microcontroller.

\subsubsection{Battery Voltage Sensor}

The battery has sufficient capacity to power the SN throughout its estimated lifetime $(\approx 1$ year) however, in subzero temperatures, the battery (SAFT: LS14500) can lose nearly half its capacity $\left(\approx 50 \%\right.$ at $\left.-40^{\circ} \mathrm{C}\right)$. A voltage sensor integrated into the microcontroller (Texas Instruments: MSP432P401R) allows the system to keep an eye on the battery and evaluate its performance under various operating temperatures.

\subsection{Summary}

This chapter explained our case study, described the application requirements, and covered the design of our solution at the system level and down to the SN's internal modules. Also, the custom designed sensors were presented in light of the application at hand. 


\section{Chapter 4}

\section{Implementation and Testing}

In this chapter, we discuss our system's implementation, the field test, and its results. The wireless communication, embedded sensors, power consumption, and the protective package are all crucial design aspects that were tested and discussed here. A total of three site visits in Fort McMurray Alberta Canada, over a five year period and in-house testing are covered in this chapter. Most of the test results were retrieved through our cloud hosted dashboard as shown in Figure 4.1 and Figure 4.2.

The dashboard can be customized with any combination of onboard sensors and reading can be plotted in real-time on many available widgets and charts. Addition-

ally, all data can be exported for post processing and further analysis. The dashboard is open source and was provided as a service [103] 


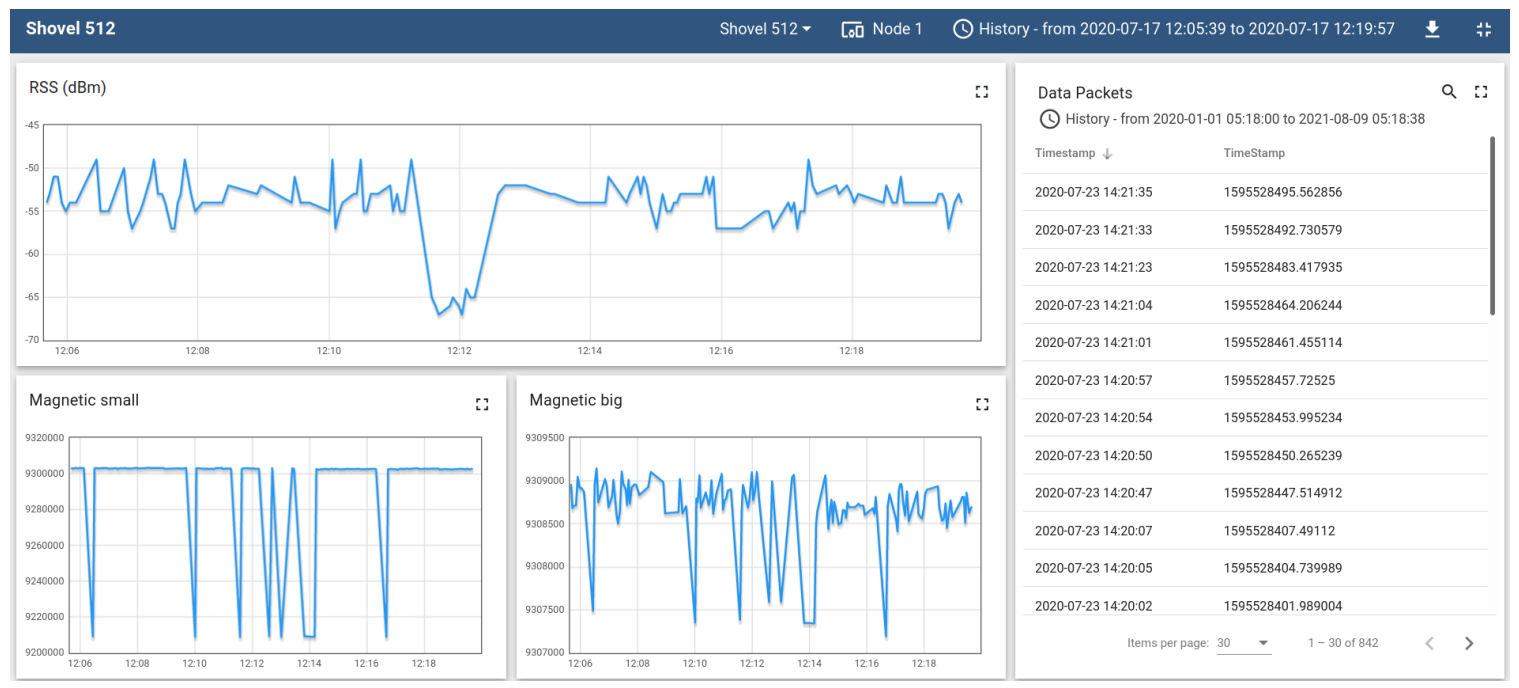

Figure 4.1: The cloud hosted dashboard for displaying sensor readings in real-time. Top part.

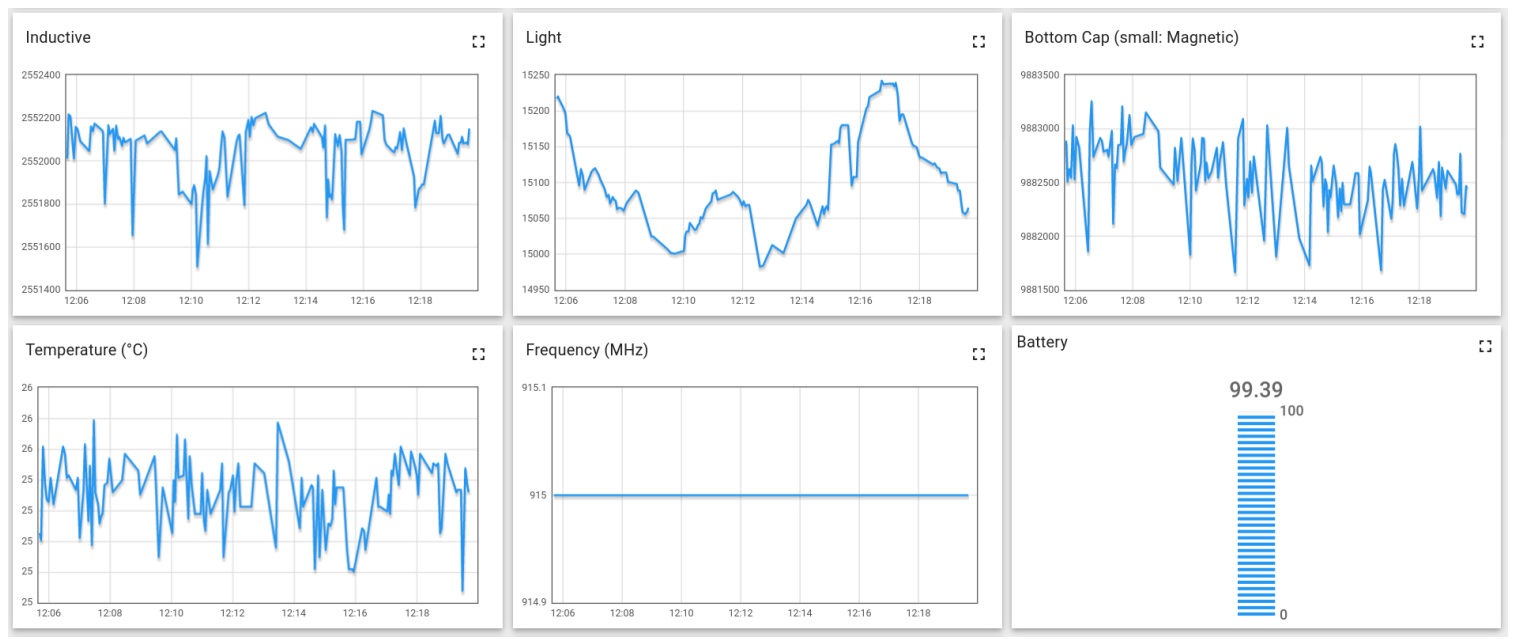

Figure 4.2: The cloud hosted dashboard for displaying sensor readings in real-time. Bottom part. 


\subsection{Package Design}

The harsh operating conditions of open-pit mining posed a number of challenges to our hardware. The protective package must withstand extreme temperatures, vibrations, mechanical shocks. Moreover, the package must be transparent for electromagnetic waves to propagate through it with minimal attenuation. Such requirements limited the materials to be used in the package to only those with low permittivity denoted by $\left(\varepsilon_{r}\right)$.

Mining operations usually take place in remote areas with extreme weather conditions. For example, in Alberta, Canada, one of the largest oil sands mining operations is carried out in Fort McMurray, where temperatures range from a minimum of $-45^{\circ} \mathrm{C}$ in winter to a maximum of $36^{\circ} \mathrm{C}$ in the summer. Figure 4.3 shows the Probability Mass Function (PMF) of extreme (maximum and minimum) daily temperatures on record between 1955 and 2016 in Fort McMurray [104].

The electric rope shovel used in mining oil sands is a giant piece of machinery, as shown in Figure 4.4. With a payload of approximately a $100 \mathrm{t}$ (metric ton), it is capable of loading a $400 \mathrm{t}$ truck in four passes. The shovel scrapes and breaks the ore with significant force resulting in amplified levels of vibrations and mechanical shocks at its teeth and bucket adapters. It is reasonable to expect that the SN will be subject to these vibrations and shocks during operation.

The package design, as shown in Figure 4.5, has three components: the silicon rubber compound, the FR10 plate, and the fibreglass sleeve. First, the silicon rubber compound provided electronics and battery cells with protection against vibrations, mechanical shocks, and extreme temperatures. The properties of the chosen compound (MoldMax: 14NV) are listed in Table 4.1. Second, the FR10 plate is an 


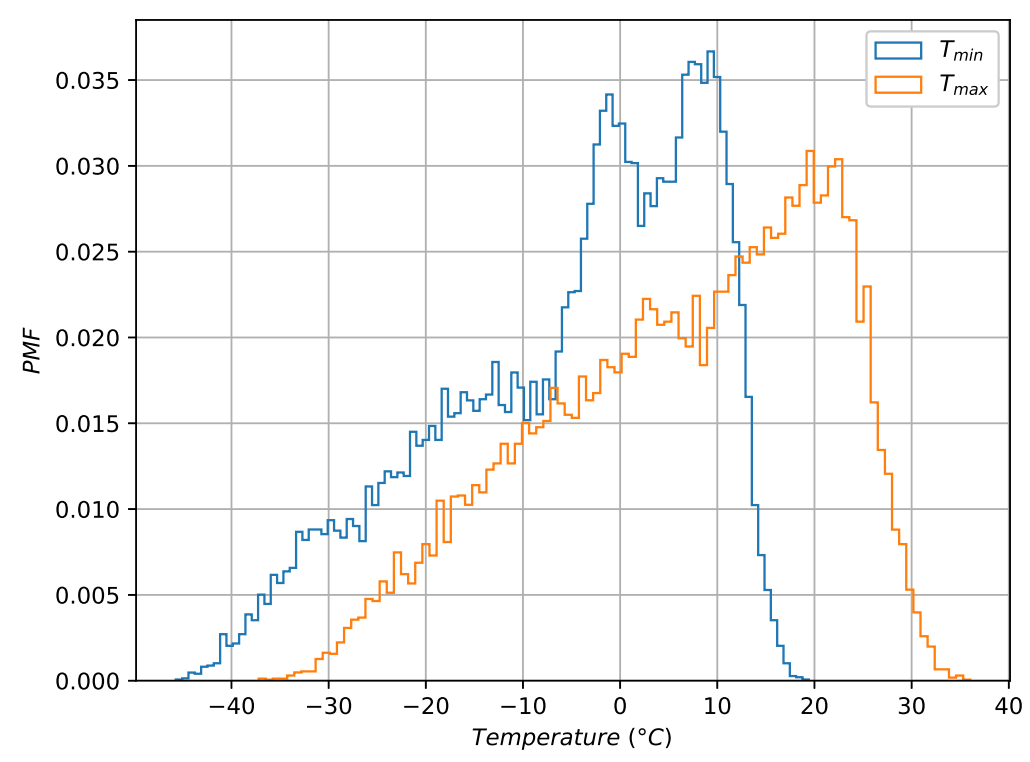

Figure 4.3: Probability Mass Function of daily extreme temperatures (maximum and minimum) records in Fort McMurray between 1955 and 2016 [104].

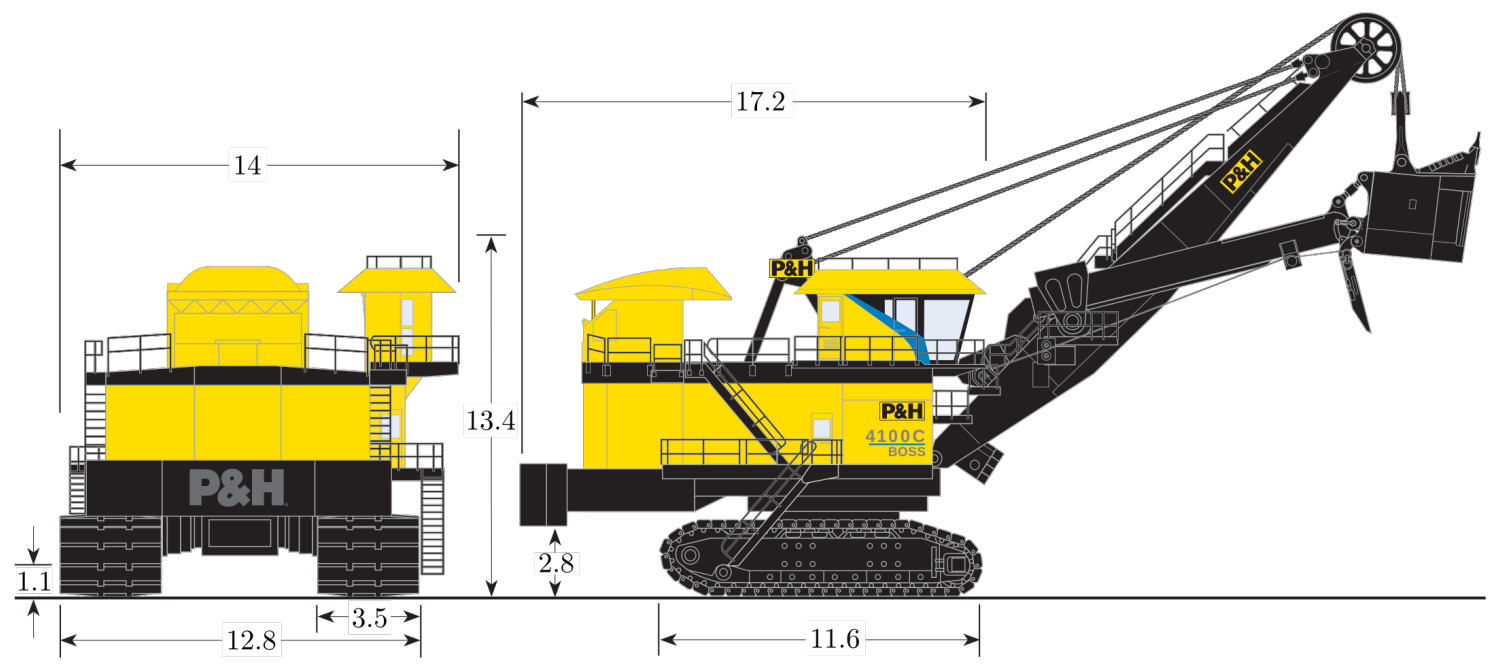

Figure 4.4: Dimensions $(\mathrm{m})$ of the electric rope shovel [2]. 


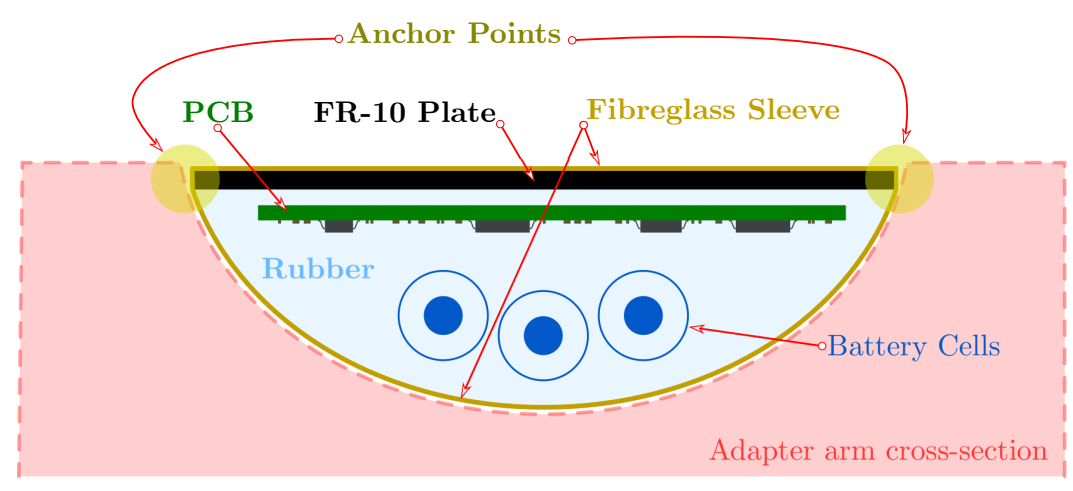

Figure 4.5: An illustration of the package.

Table 4.1: Properties of the encapsulating silicone rubber (MoldMax: 14NV).

\begin{tabular}{|c|c|c|}
\hline Property & Value & unit \\
\hline Tensile Strength & 3.38 & $\mathrm{MPa}$ \\
\hline 100\% Modulus & 0.241 & $\mathrm{MPa}$ \\
\hline Elongation @ Break & 600 & $\%$ \\
\hline Shore Hardness & $14 A$ & $\mathrm{~N} / \mathrm{A}$ \\
\hline Useful Temperature & -54 to 204 & ${ }^{\circ} \mathrm{C}$ \\
\hline Dielectric Strength & 13.002 & $\mathrm{MV} / \mathrm{m}$ \\
\hline Relative Permittivity $\left(\varepsilon_{r}\right) @ 100 \mathrm{~Hz}$ & 3.29 & $\mathrm{~N} / \mathrm{A}$ \\
\hline Dissipation Factor $(D F) @ 100 \mathrm{~Hz}$ & 0.005 & $\mathrm{~N} / \mathrm{A}$ \\
\hline Volume Resistivity & $5.83 \times 10^{13}$ & $\Omega \mathrm{cm}$ \\
\hline Thermal Conductivity & 0.21 & $\mathrm{~W} / \mathrm{mK}$ \\
\hline
\end{tabular}

industry-standard fibreglass plate known for its superior strength. It also has similar permittivity to the FR4 used in most circuit boards. Third, the fibreglass sleeve was used to hold the package together while being a proper substrate for gluing the SN to the adapter's steel cavity with the chosen impact-resistant adhesive (Permabond: TA4246). 
For our SN to withstand the extreme temperatures of the mine, we had to review temperature tolerance of all off-the-shelf components. For the maximum operating temperature, most components available on the market have an operating temperature of $85^{\circ} \mathrm{C}$ or more. However, to ensure the sensor's reliability at very low temperatures, only components with operating temperatures of $-40^{\circ} \mathrm{C}$ or less have been used in the circuit design. Finding components that remain functional below $-40{ }^{\circ} \mathrm{C}$ is difficult and not cost-effective. Thus, we limited our requirement to $-40{ }^{\circ} \mathrm{C}$ after considering the very small probability of temperatures less than $-40^{\circ} \mathrm{C}$ occurring which is 0.005387 . Further on, the low thermal conductivity $(0.21 \mathrm{~W} / \mathrm{mK})$ of the silicon rubber compound added another layer of protection against extreme temperatures.

The silicon rubber helps the SN withstand vibrations by dampening them. Worth mentioning that this particular rubber compound is able to maintain its softness and flexibility even at low temperatures, as shown in Table 4.1. Moreover, in the event of a mechanical shock, the rubber distributes impact forces evenly, minimizing pressure on the internal components.

The package had to have electromagnetic properties that allow for the propagation of waves with very low attenuation. As shown in Table 4.1, the rubber has a low dissipation factor $(D F=0.005)$ and relative permittivity $\left(\varepsilon_{r}=3.29\right)$. Furthermore, the FR10 plate had even lower ratings for the $D F$ and $\varepsilon_{r}$.

\subsubsection{Impact Test}

The SN's package was initially tested using casual mechanically abusive tests. The SN remained functional after these tests. Furthermore, we administered an impact test by dropping a $15 \mathrm{~kg}$ steel box on the packaged SN from a height of $1.2 \mathrm{~m}$. The top 
surface of the SN rested $20 \mathrm{~mm}$ above the concrete floor, which means the steel box travelled for $1.18 \mathrm{~m}$ before hitting the SN. The impact energy is the kinetic energy (KE) of the steel box at the moment of impact, which is equal to its potential energy $(\mathrm{PE})$ at the height from which it was dropped. After neglecting air resistance, the classical mechanics calculation of the impact energy is $K E=P E=m g h=173.46 \mathrm{~J}$. Where $m$ is the mass of the steel box, $h$ is the height from which it was dropped, and $g$ is the acceleration due to gravity. In order to calculate the impact force, we needed to know how far the steel box travelled after impact. By observing the moment of impact we concluded that the steel box completely stops at impact without bouncing back (i.e., $\triangle K E=173.46$ ). Also, on close inspection of the SN after impact, there was no considerable deformation in the SN's package and that leads us to assume a very small travel distance $\left(d_{t} \leq 2 \mathrm{~mm}\right)$. This puts a lower bound on the impact force $\left(F_{\text {imp }}\right)$, as shown in equation 4.1 .

$$
F_{i m p}=\frac{\triangle K E}{d_{t}} \geq 86730 N
$$

Therefore, we can confirm that the SN experienced an impact force $\left(F_{\text {imp }}\right)$ of at least 8.844 ton-force (metric). All tested SNs survived the impact test without any loss in functionality or performance.

\subsubsection{Packaging Process}

This section discusses the packaging process of SNs. The quantities of packaged SNs for our work were only enough to monitor nine shovel teeth. Therefore described in this section is a very labour-intensive process. It is reasonable to assume that the techniques presented here can be mass-produced economically in a manufacturing 
facility with the proper tooling and fixtures.

With the PCB shown in Figure 4.6 we know from previous trials that directly pouring the rubber onto the board will permeate under the small passive components and strip them off the board should the rubber be pulled off. Since our development work involved investigating faults, we needed access to the board for troubleshooting. Thus we pre-coated the PCB with liquid electric tape as shown in Figures 4.7 and 4.8, which enabled the rubber to be removed later if needed without compromising the integrity of the PCB assembly. Since the liquid electric tape is a relatively thin layer, it will be easily stripped and torn during removal before exerting too much tensile force on the solder joints.

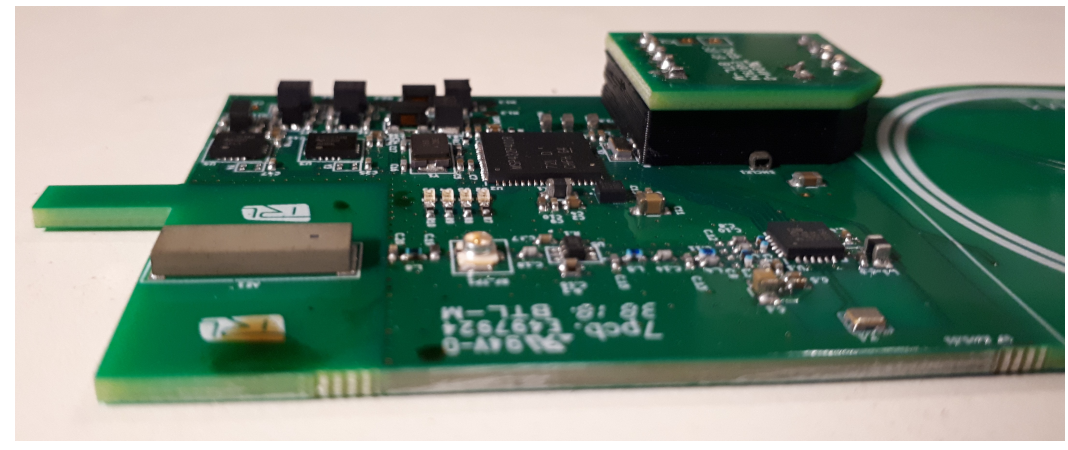

Figure 4.6: Full PCB closeup showing the recessed PCB housing the programming port, the power switch, and the LDR.

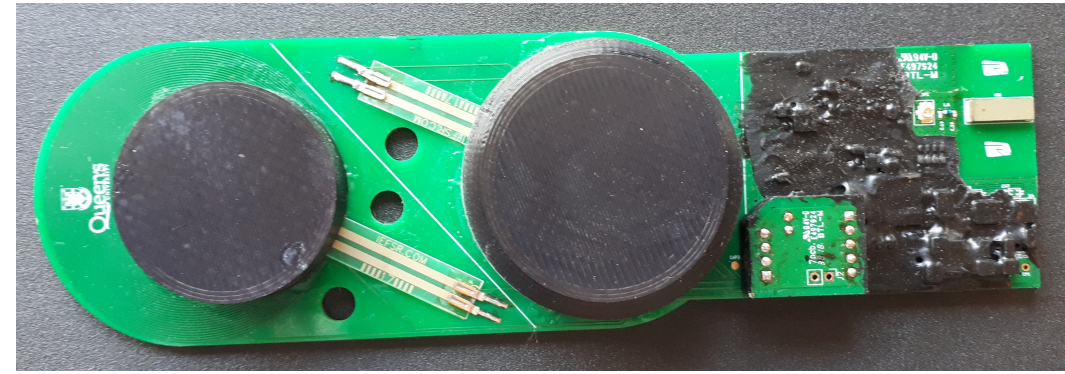

Figure 4.7: Full PCB after coating with liquid electrical tape. 


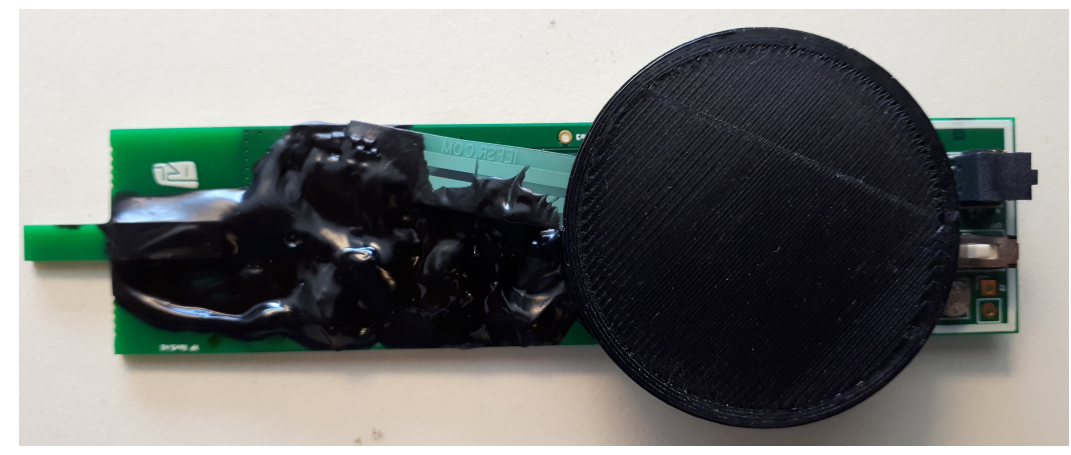

Figure 4.8: Reduced PCB after coating with liquid electrical tape.

In Figure 4.9, we see an assembled batch of SNs being prepped for packaging. The nodes shown are at different stages where some of them had their RF tuning port exposed to facilitate impedance measurement while the one in the top right corner is completely coated with liquid tape, glued to the FR10 plate and soldered to the battery and the poly-carbonate support with its capacitive sensor.

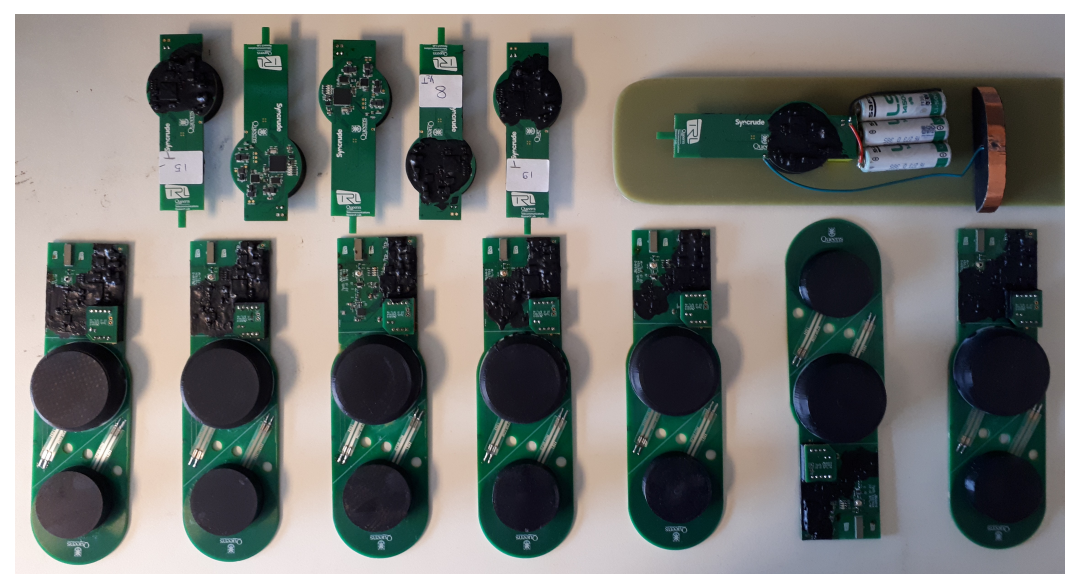

Figure 4.9: Assembled batch of SNs before packaging.

The rubber mould in Figure 4.10 was designed in reference to the CAD model of the adapter. Afterwards, the mould was 3D printed from PLA plastic and postprocessed to smooth its surface. Post-processing involved staggered sanding with 


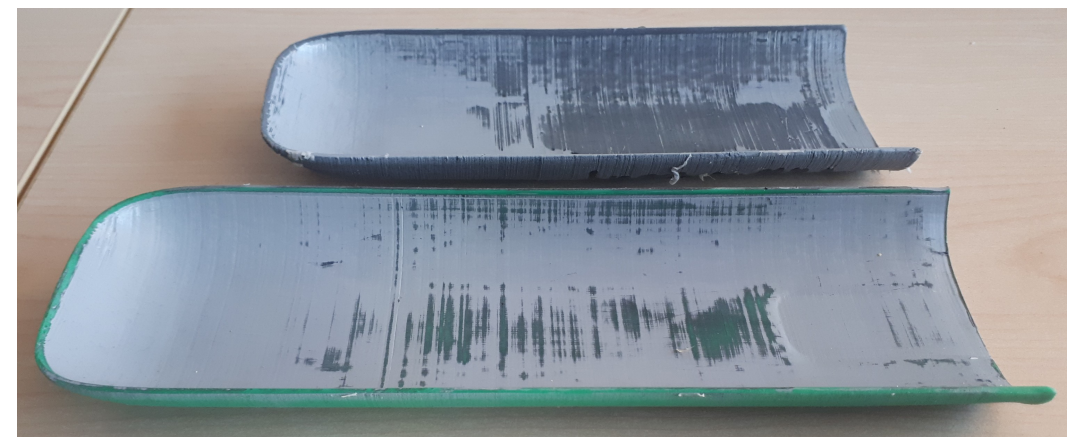

Figure 4.10: The 3D printed rubber mould after post processing.

increasing values of sandpaper grit, coating with a primer (Design Masters: 645 Primer) and sanding again. The smooth surface achieved allowed for easy removal of the cured rubber.

Before potting the hardware assembly in rubber, a few steps were required to prepare the mould. First, the mould had three coats of a mould-release agent applied (Mann release technologies: Ease Release 200). Second, the mould was glued to the FR10 plate assembly using hot glue to seal the seams. Third, the entire assembly was tightly held together with plastic wrap and rubber bands. Finally, the mould was suspended vertically as shown in Figure 4.11, the rubber compound was premixed, poured into the top opening of the mould, and left to cure as shown in Figure 4.12.

After the rubber cured, the wrapping material and the plastic mould were removed. Then, the FR10 plate was trimmed to align with the poly-carbonate support. A partially potted SNs before trimming is shown in Figure 4.13 to demonstrate this step.

Figure 4.14 shows the SNs after rubber potting and trimming. This is before each one was inserted into a fibreglass sleeve as shown in Figure 4.15. The sleeve was trimmed accordingly and folded at the edges and held with a porous medical fabric 


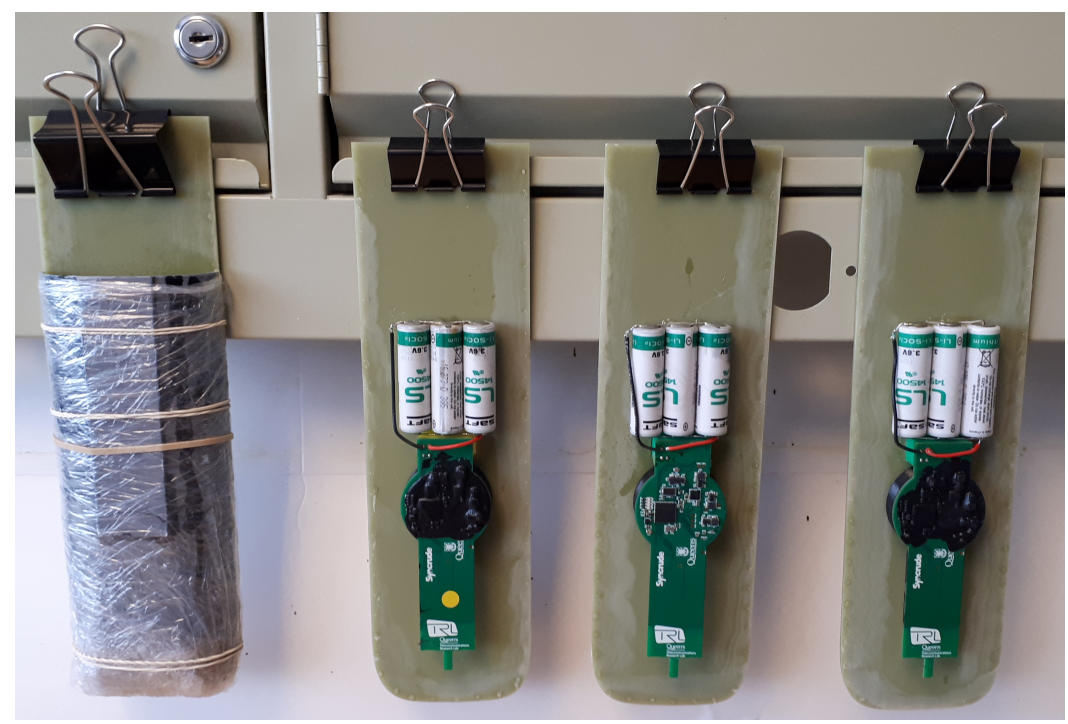

Figure 4.11: Rubber pouring setup.

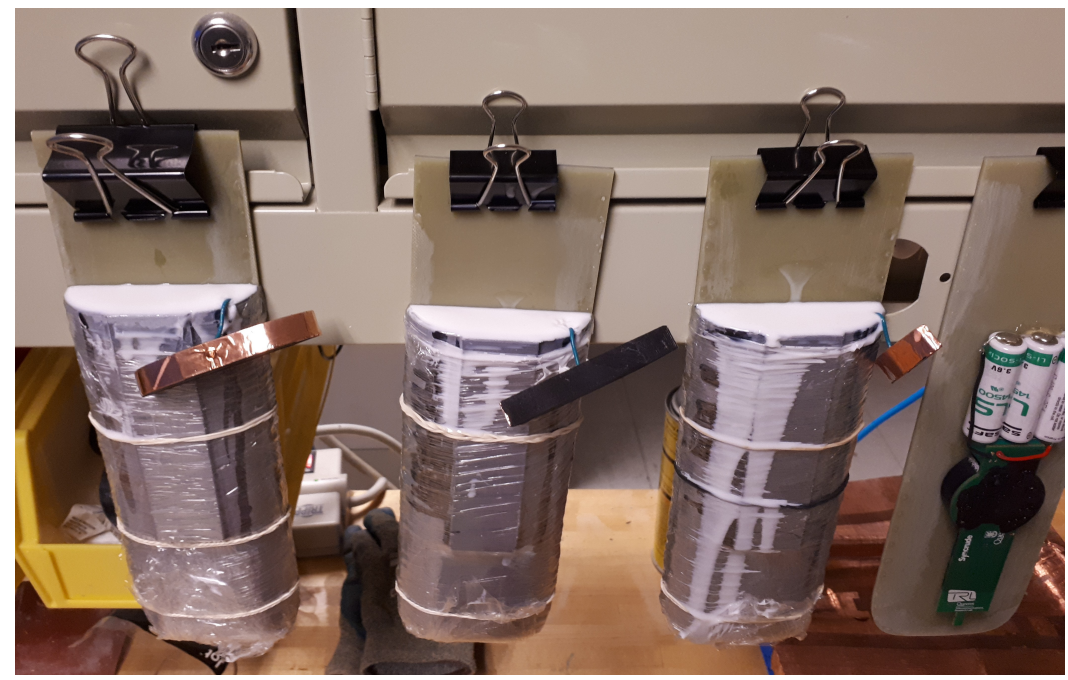

Figure 4.12: Full PCB after coating with liquid electrical tape.

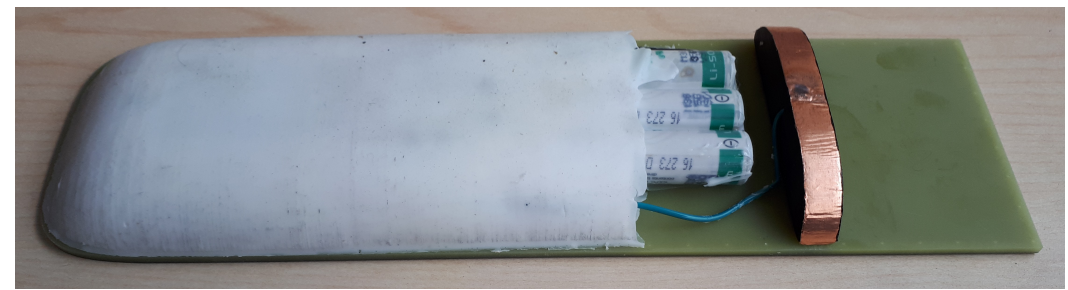

Figure 4.13: Partially potted SNs after curing the rubber. 


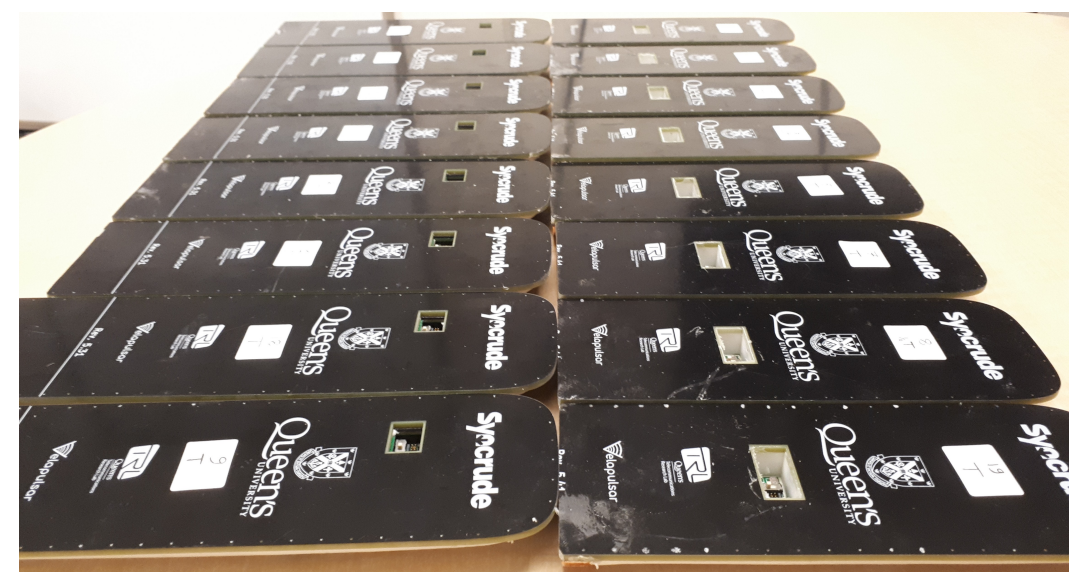

Figure 4.14: A batch of SNs before being covered with the fibreglass sleeve.

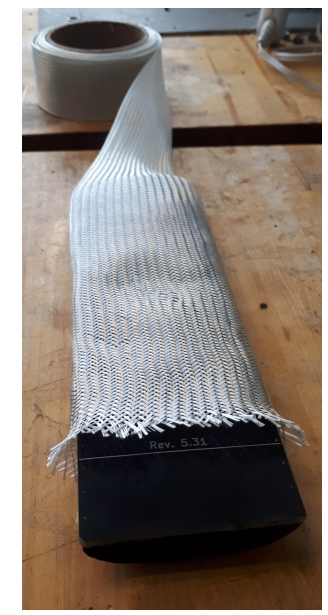

Figure 4.15: An SN being covered with the fibreglass sleeve.

tape. The chosen tape helped the fibreglass in absorbing resin at the folded edges. A small opening in the fibreglass fabric was made, as shown in Figure 4.16 to provide access to the power switch, the programming port, and allow ambient light to reach the LDR. Then the package was coated with a high-temperature epoxy resin (Fibre Glast: 3000 Resin and 3120 Hardener), wrapped tightly with plastic wrap ensuring there were no wrinkles in the fibreglass. and left to cure. The finished package is shown in Figure 4.17. 


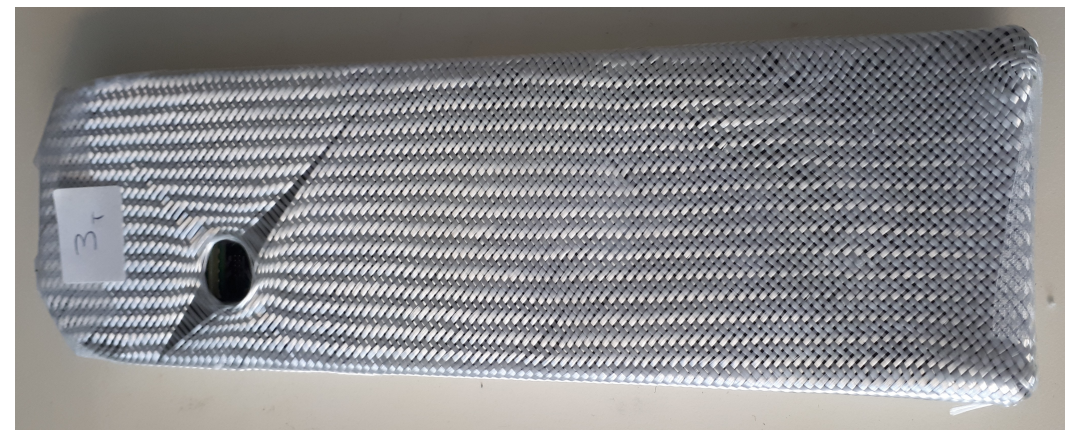

Figure 4.16: The opening in the fibreglass weave for access to the power switch, the programming port, and the LDR. Before coating with resin.

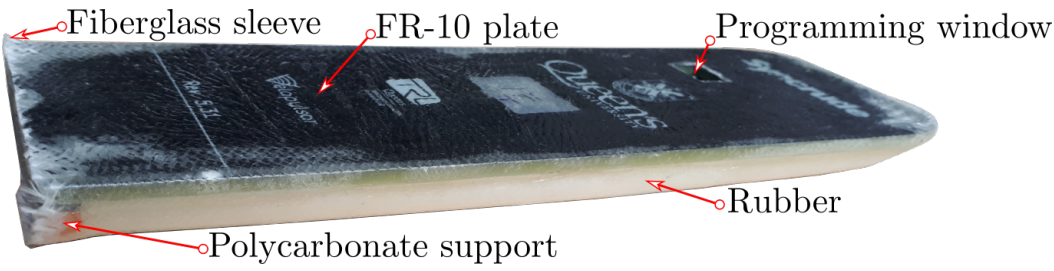

Figure 4.17: Fully packaged SNs.

Afterwards, we proceeded with testing to verify the performance of the packaging under various types of mechanical loads.

\subsection{Communication}

We performed several types of tests to evaluate the performance of the wireless connection between the SNs and the sink. Our performance metric is the measured RSS at the sink.

\subsubsection{Range}

An experiment was conducted to confirm that the communication range of the SNs from inside the adapter's cavity will exceed $25 \mathrm{~m}$. We used the cavity mock-up created to tune the matching network of the antenna. An SN was programmed to transmit 


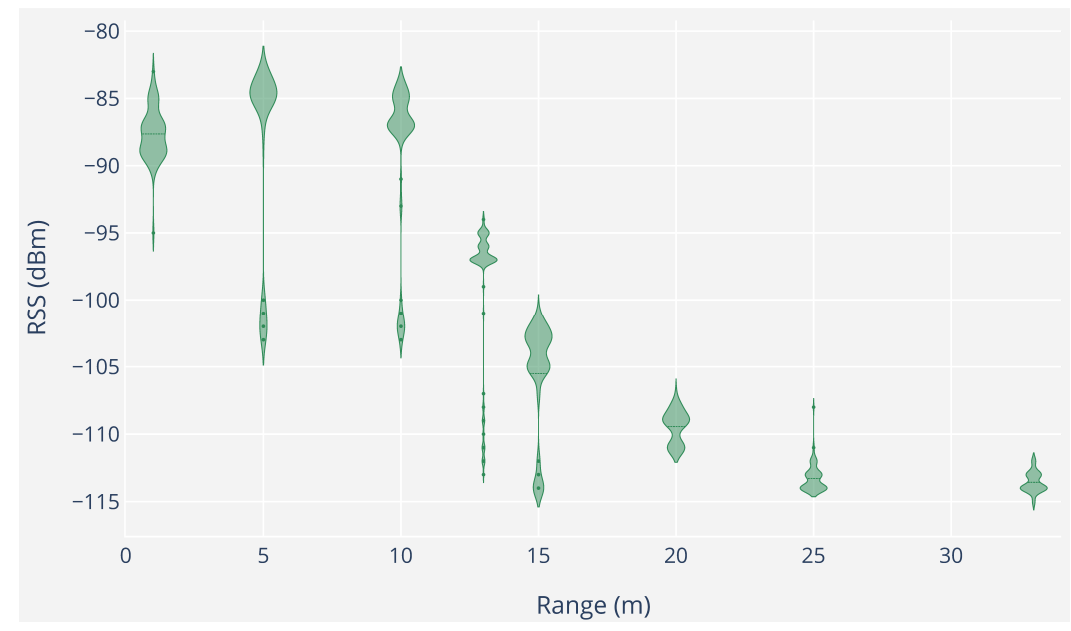

Figure 4.18: A violin plot of the communication range vs. RSS.

data packets continuously and then was placed inside the cavity mock-up and covered with the aluminum sheet with a $3 \mathrm{~mm}$ gap between the sheet and the cavity mock-up. The sink was used to measure the RSS of the SNs at various distances. The sink used a Yagi array pointed directly to the SNs at all times. The measurements are shown in Figure 4.18.

The violin plot in Figure 4.18 shows the distribution of RSS reading at various ranges. We assumed the multi-modality and the asymmetry of the distributions is attributed to the uncontrolled indoor environment in which this experiment was carried out (a long hallway). This is in line with the data presented in [105], which shows a similar statistical character for the same environment. In other words, if it has taken place in an anechoic chamber, single-mode distributions with high centrality would have shown up in the results.

As shown in Figure 4.18, the communication range exceeds our requirement of $25 \mathrm{~m}$ with enough margin $(25 \mathrm{dBm})$ in the link budget considering that the receiver sensitivity is at $-140 \mathrm{dBm}$. 


\subsubsection{Antenna Matching}

As explained in section 3.4.3, the matching was performed to improve the RSS at the sink. Figure 4.19 shows the results of an experiment undertaken to confirm the effect of matching on the RSS at the sink. For both PCB designs, we compared the RSS of a node with a matched antenna (tuned) versus a node with an unmatched antenna (untuned).

Ideally, this test should be performed inside an anechoic chamber, yet the scope of this study did not justify the cost of renting one. However, extra care was taken in isolating all other factors of potential influence on the RSS. For example, we used the same hardware revision for both SNs (i.e., same circuit design, PCB layout, and manufactured batch), and we outsourced the PCB assembly to reduce performance variations from manual in-house assembly. Additionally, we maintained the physical state of the lab environment throughout the experiment (i.e., no moving objects or people were around during the experiment).

As we notice in Figure 4.19, tuned nodes did not have any RSS advantage over untuned nodes when placed outside the cavity. In fact, for the full PCB, the untuned nodes showed a $5 \mathrm{dBm}$ increase in RSS over the tuned nodes. This is because the tuned nodes had their antennas matched with the cavity which means they are unmatched in the "Outside" scenario. The other scenarios are the "Open Cavity" and "Closed Cavity" where the nodes are placed inside the cavity mock-up without and with the aluminum sheet cover, respectively. The tuned nodes show a clear advantage over the untuned nodes (up to $18 \mathrm{dBm}$ ). This supports the efficacy of antenna matching in enhancing the RSS of an SNs communicating from a non-hermetic metallic container which was the focus of an earlier work [34]. 


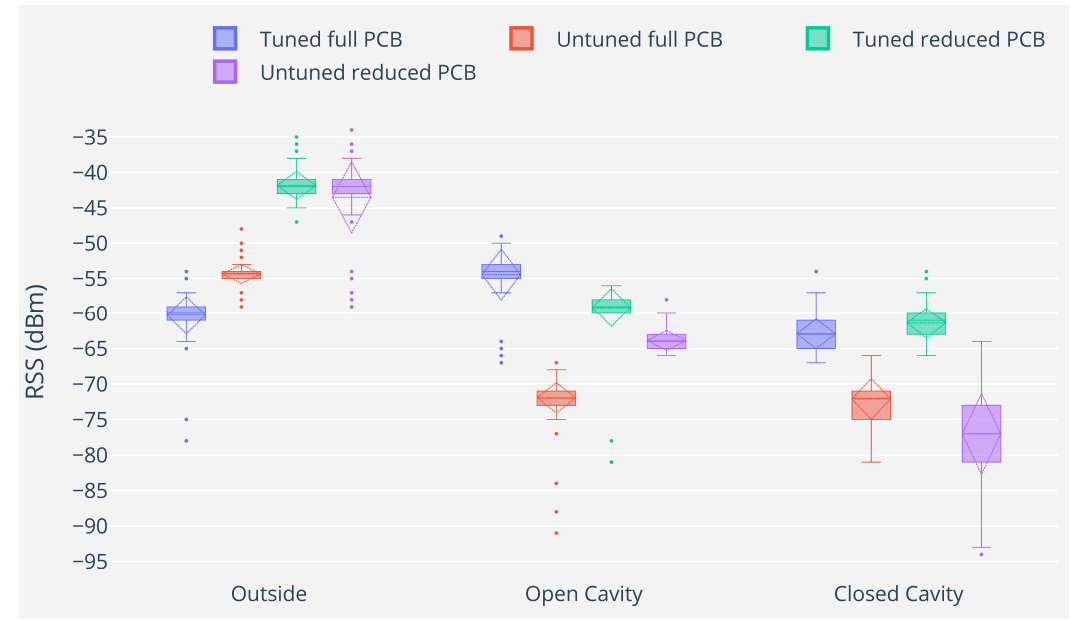

Figure 4.19: Tuned versus untuned nodes in three scenarios: Outside (when the node is outside the cavity and fully exposed), Open Cavity(when the node is inside the cavity but without a cover), Closed Cavity (when the cavity is covered)

\subsubsection{Buried Sensor Node Communication}

This test was performed to validate the reliability of the wireless link when the SNs is buried under a pile of sand or rocks.

The first test used an exposed, untuned PCB Rev. 5.31 SNs. It was intended to stress test the wireless link. In this test, the $\mathrm{SN}_{\mathrm{s}}$ was placed on the ground. Then, the shovel dumped five loads of soft sand on top of it, as shown in Figure 4.20. During this time, the signal strength was being monitored. Throughout the test, the sensor was functional, and the RSS was above $-132 \mathrm{dBm}$.

In the second test, we put another SNs in a strong wooden box (made from 2X4 inch construction wood) to protect it from being crushed by the rocks falling from the shovel. Wood was used since we needed a non-metallic enclosure to allow the wireless signal to propagate. Then the box with the SNs inside was thrown in the shovel's bucket and the shovel proceeded to load a hauler truck with four loads of 


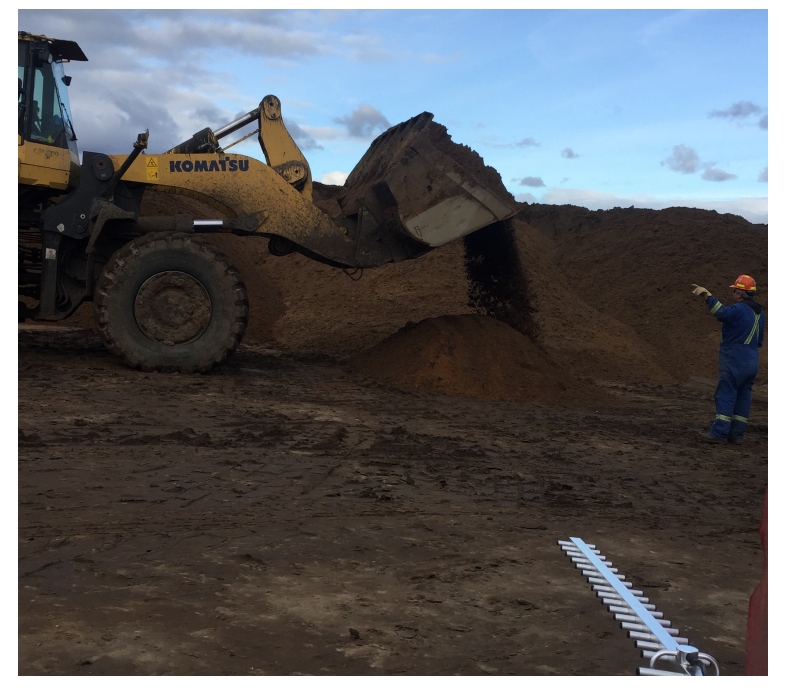

Figure 4.20: The SNs is on the ground underneath the sand being poured. Receiving antenna is $25 \mathrm{~m}$ away in the lower right corner

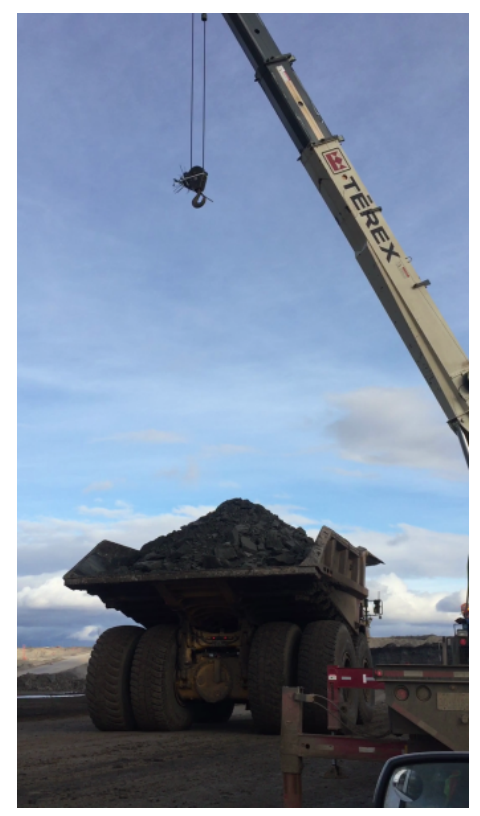

Figure 4.21: The SN is buried inside the truck's load. The sink and its antenna are hanging from an overhead crane on top of the hauler truck. 
rocks estimated at 400 metric ton. Unfortunately, after the second load was dumped into the truck, the signal stopped suddenly, indicating a crushed SNs. Even when we got the sink closer to the SNs by attaching it to an overhead crane, as shown in Figure 4.21, we still could not pick up a signal.

\subsection{Sensing}

\subsubsection{Proximity Sensors}

Field testing the wireless sensors required stopping an operational shovel for about an hour to install the sensors on its adapters. The downtime of the shovel translates into a considerable oil production loss. Hence, the field test had to be planned carefully and executed quickly.

The field test started at the on site workshop where a couple of sensor nodes were installed on an adapter. Then the adapter was repeatedly attached/detached to an idle bucket while sensor readings were collected and compared to the timed events log created by the field team. Figure 4.22 shows an example of the data collected from the workshop test. The readings are expressed as raw outputs from quantizers. It is worth mentioning that in a few cases, the adapter would be partially detached while some or all the sensors are indicating otherwise. This is because in these few cases, the proximity sensors were still able to detect the bucket's presence even when the adapter was not fully inserted. This means that individual sensors may not detect partial detachments but will reliably detect a complete detachment.

After the workshop test was concluded, the system was ready for a test on an operational shovel. A low priority shovel was assigned and sensor-equipped adapters were installed on its bucket as shown in Figure 4.23. In order to simplify and speed 


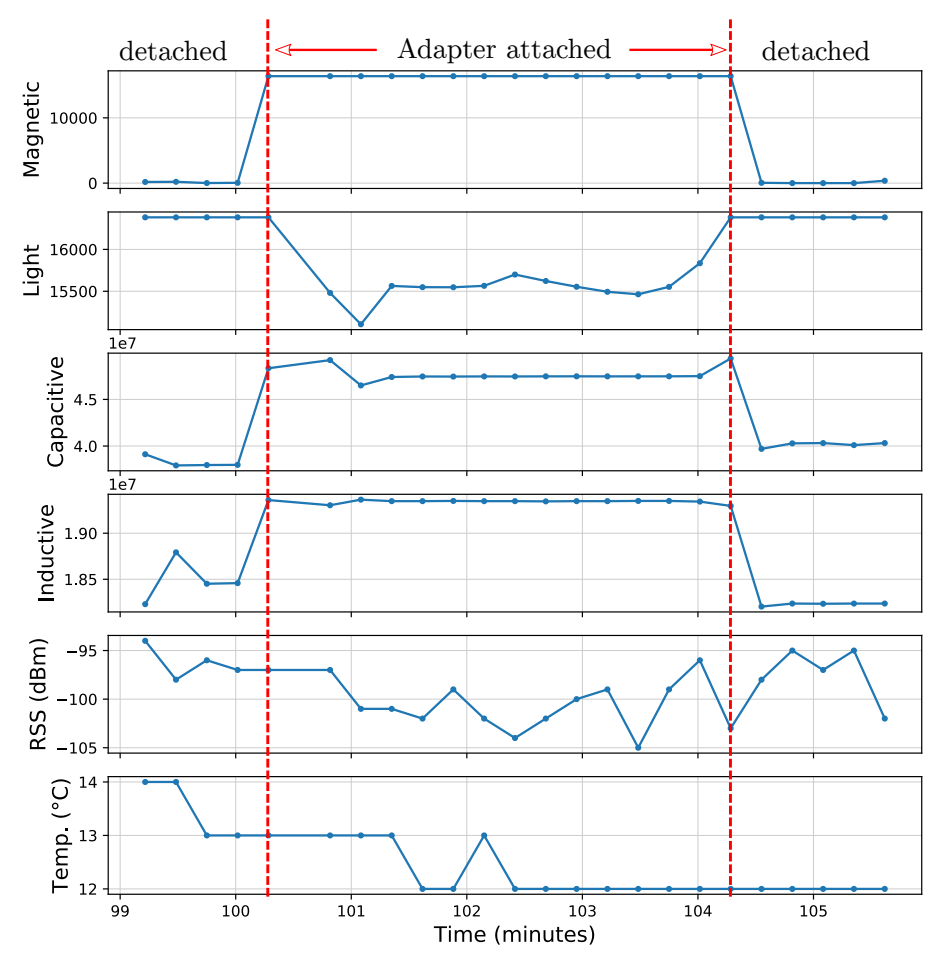

Figure 4.22: Sensor readings from an attaching/detaching test in the workshop.

up installation, strong mounting neodymium magnets (K\&J Magnetics: MM-A-48) were placed inside the sensor's package. These magnets allowed the SNs to be latched inside the adapter's cavity.

After installing the SNs, the sink node was mounted on a railing right next to the operator's cabin. A Yagi array was used with the LoRa ${ }^{\mathrm{TM}}$ transceiver of the sink node. It boosted the wireless link budget by about $14 \mathrm{~dB}$. The location of the sink node relative to the bucket is shown in Figure 4.24. The range is approximately $25 \mathrm{~m}$ and with a highly sensitive receiver (down to $-142.5 \mathrm{dBm}$ ) the wireless link budget was enough for a reliable connection.

The sensors were up and running before being installed on the adapters. Initially, 


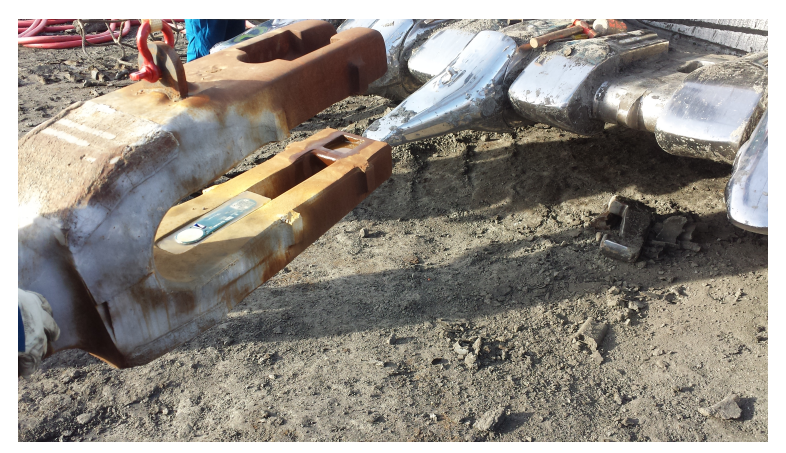

Figure 4.23: The sensor node inside the adapter while it is lifted and being attached to the bucket on an operational shovel.

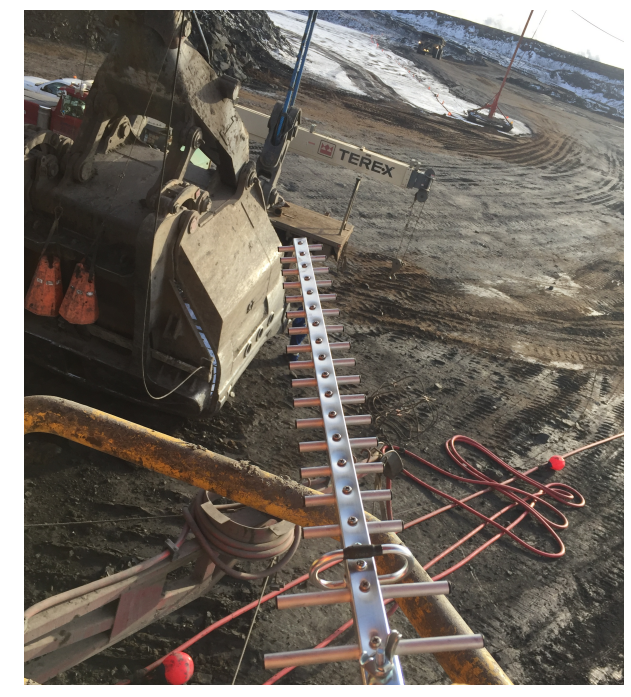

Figure 4.24: A photo from the sink node's location on a rail next to the cabin. The LoRa ${ }^{\mathrm{TM}}$ transceiver used a Yagi array for its high gain and directivity which added an extra $14 \mathrm{~dB}$ to the wireless link budget.

the sensors were inside the cabin of a pickup truck with its heat turned on. This is reflected in the room temperature readings during the first few minutes $\left(\approx 23{ }^{\circ} \mathrm{C}\right)$ followed by a downward trend towards the ambient air temperature on that day $(\approx 8$ $\left.{ }^{\circ} \mathrm{C}\right)$. It took more than 20 minutes for the core temperature of the sensor node to reach that of the ambient air. This is due to the low thermal conductivity of the packaging rubber $(0.21 \mathrm{~W} / \mathrm{mK})$. After digging started (around minute 67 ), the friction caused 


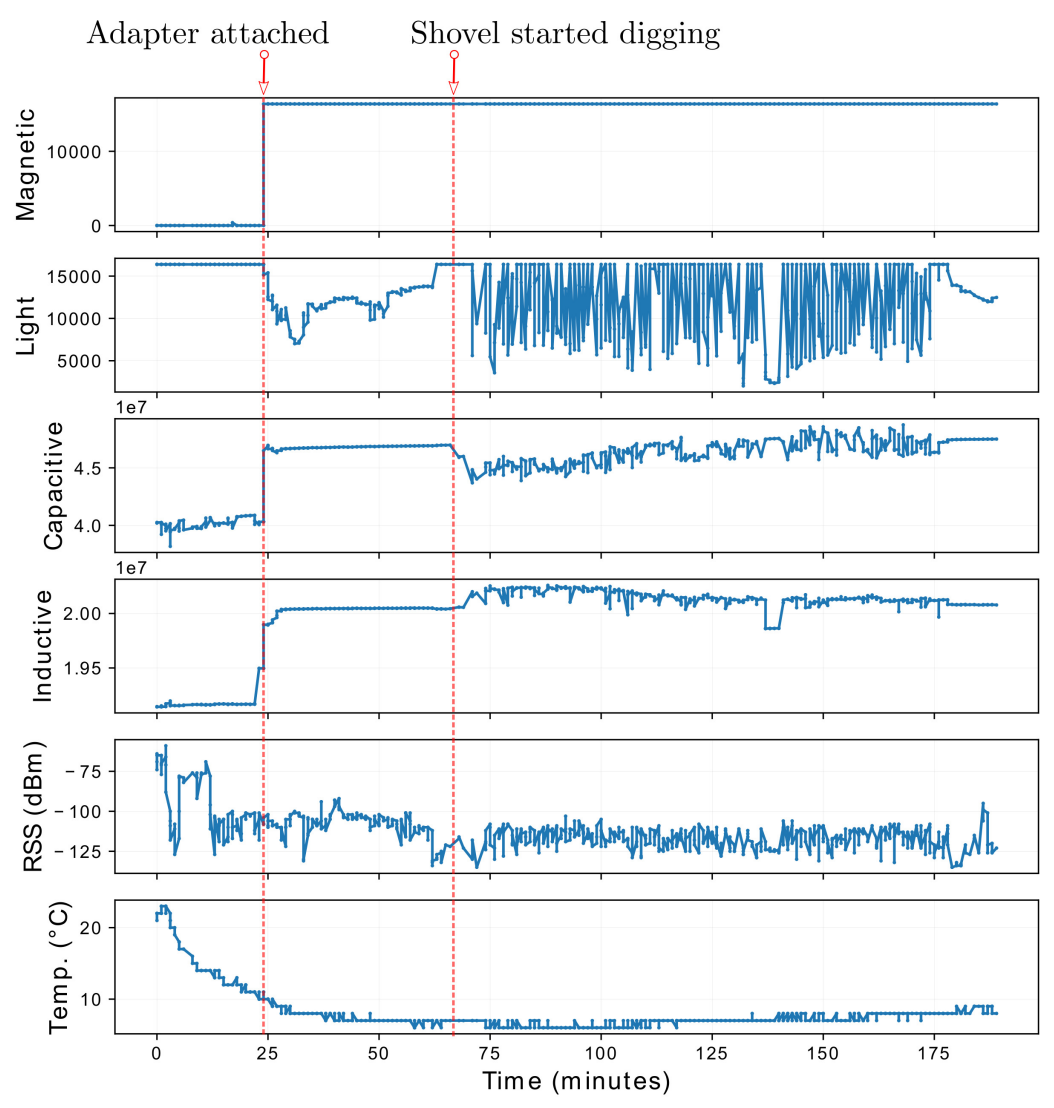

Figure 4.25: Sensor data collected from an operational shovel during the field test.

the adapters/teeth to heat up. We can notice the slowly increasing core temperature as the heat propagates to the inner core of the sensor node.

The light sensor performed as expected in the workshop test as shown in Figure 4.22. The same expected output was observed later when the adapter got attached to the bucket and before the shovel started digging. However, when the digging started, the readings fluctuated significantly as shown in Figure 4.25.

The magnetic sensor showed a steady response in the workshop and on the operational shovel. This sensor has the most stable output. The distinction between both states (attached/detached) of the adapter is very clear. The reason for this clear 
distinction is that the neodymium magnet inside this sensor was chosen so that the anticipated proximity range of the bucket would cause it to saturate the FSR. This is explained in more details in [35].

Capacitive and inductive sensors exhibit a similar behaviour. Once again, their output before the shovel started digging is very close to their output in the workshop. After digging started, we notice marginal fluctuations in their readings; however, there is still a clear distinction between the two states (attached/detached). These fluctuations are attributed to the wiggling motion of the adapter with respect to the bucket. Since both of these sensors have a sub-millimetre resolution, they were able to pickup the wiggling motion.

During the numerous attachment/detachment tests in the workshop, the detection rate was $100 \%$ for all the proximity sensors except the light sensor, its rate was $80 \%$. As for the active shovel test, the light sensor failed to detect attachment while the capacitive and inductive sensors succeeded with a reasonable margin and the magnetic sensor with an excellent margin.

Status of individual adapters are displayed on the cabin tablet as shown in Figure 4.26. It is constantly receiving sensory data in real-time from the sink node through a Wi-Fi connection. The tablet was added to the system to provide a convenient userfriendly, graphical interface for the operator. It produces audible and visual alarms whenever a detachment is detected and specifies the location of the missing adapter.

\subsubsection{Wear-Level Sensor}

The standard model of a parallel plate capacitor with two plates is shown by equation 4.2 . 


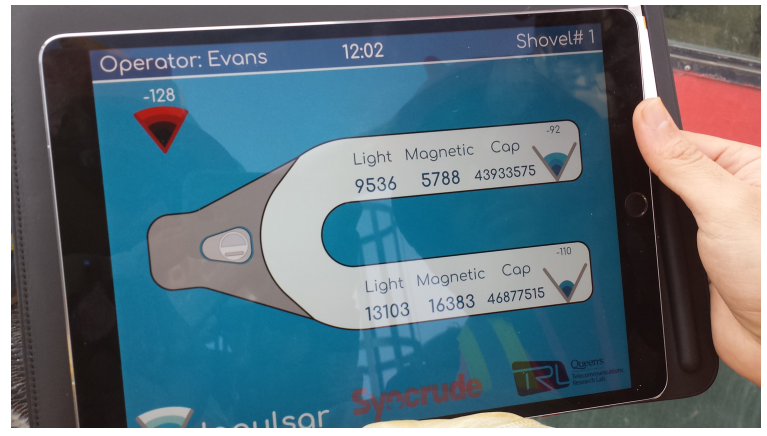

Figure 4.26: Operator dashboard on the cabin tablet. Sensor readings from both nodes are shown on their respective adapter's arm along with their RSS in dBm on the right.

$$
C_{o}=\frac{\varepsilon l w}{d}
$$

Where $\varepsilon$ is the the permittivity of the medium between the plates, $l$ and $w$ are the length and width of the capacitor plate respectively (assuming identical dimensions for both plates), $d$ is the distance between the two plates. Unfortunately, the classical model fails to account for the fringing effect. Consequently, several theoretical models were developed to approximate the fringing effect in parallel plate capacitors by multiplying $C_{o}$ by a correction factor $\beta$ such that $C \approx C_{o} \beta$ where $C$ is the total capacitance after correction. These models were evaluated and compared by [106]. Unfortunately, all models in [106] diverge from our measurements at larger probe lengths. Thus, we tried another model [107] which was built for a capacitor structure exactly similar to ours and while it was better at low probe lengths, it still diverged from our measurements at larger probe lengths. After comparing all the models in [106] and [107], we found the one developed by Hutson [108] to best approximate our measurements with a correction factor shown in equation 4.3 but, it is still not close enough to allow for accurate length measurements. 


$$
\beta_{H}=1+\frac{2 d}{\pi R} \ln \frac{8 \pi R}{d}
$$

where

$$
R=\sqrt{\frac{l w}{\pi}}
$$

Where $R$ is the radius of the capacitor plate in case it was circular, however, in our case where the plate is rectangular, $R$ is defined as a function of $l$ and $w$ as shown in equation 4.4 .

Figure4.27 shows the capacitance of the probe versus its length. Measurements were compensated for parasitic capacitance between the sensor and the CDC. Each measurement was averaged over 100 samples or more with a standard deviation $\sigma \leq 0.001 \mathrm{pF}$. The divergence of the measurements from all models as the length increases can suggest a neglected term in theoretical models that manifests in relatively large capacitor geometries. Further investigations are needed to upgrade the classical models accordingly. Consequently, to allow for an accurate length measurement from the probe's capacitance we created an empirical model for that specific probe design. In our case, the empirical model for the probe's capacitance $C_{e m p}$ is a fitted regression line $C_{e m p}=i+s l$ with an intercept $i=-8.17 \times 10^{-14}$ and a slope $s=5.13 \times 10^{-11}$. 


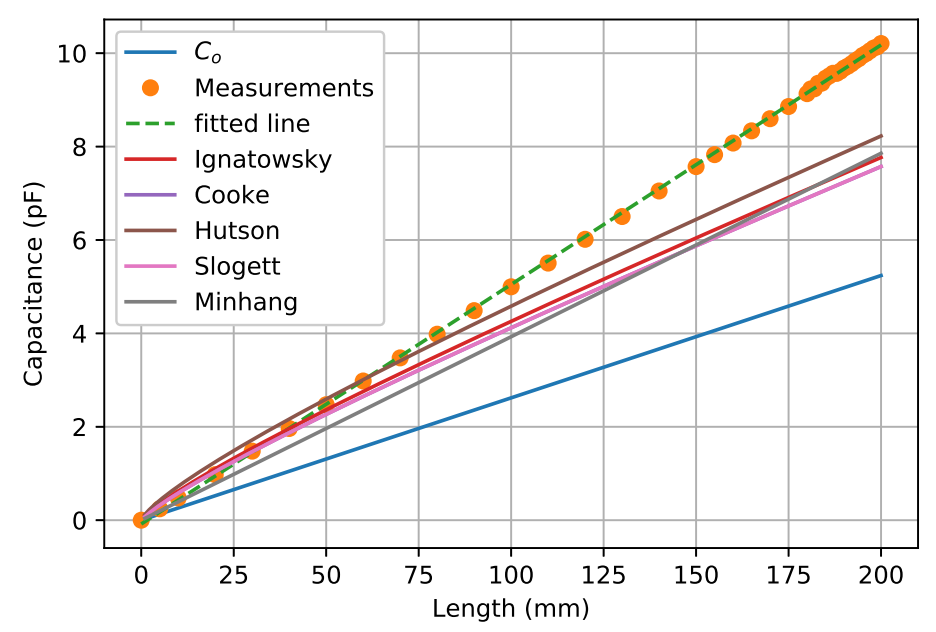

Figure 4.27: Wear level sensor capacitance vs its length.

\subsection{Power}

We measured the power consumption of the SNs using a programmable DC source (Agilent/Keysight: 66309D Mobile Communications DC source). The DC source sampled the supply voltage and current every $1 \mathrm{~ms}$.

Throughout the test, the batteries maintained a stable supply voltage of 3.61 v. The current consumption profile for a single duty cycle (idle $\rightarrow$ sense $\rightarrow$ transmit $\rightarrow$ idle) is shown in Figure 4.28. The total energy consumed per cycle is estimated at $61.94 \mathrm{~mJ}$ over a period of $866 \mathrm{~ms}$.

These results indicate that without even invoking the deep sleep mode of the microcontroller and with the 0.5 factor of safety for the battery capacity, the 9-cell battery is sufficient for a total of 2,449,790 data packets which if distributed evenly over one year would give a maximum status update delay of approximately 13 seconds. Such delay is small enough because it takes the shovel nearly four minutes to load one hauler truck. 


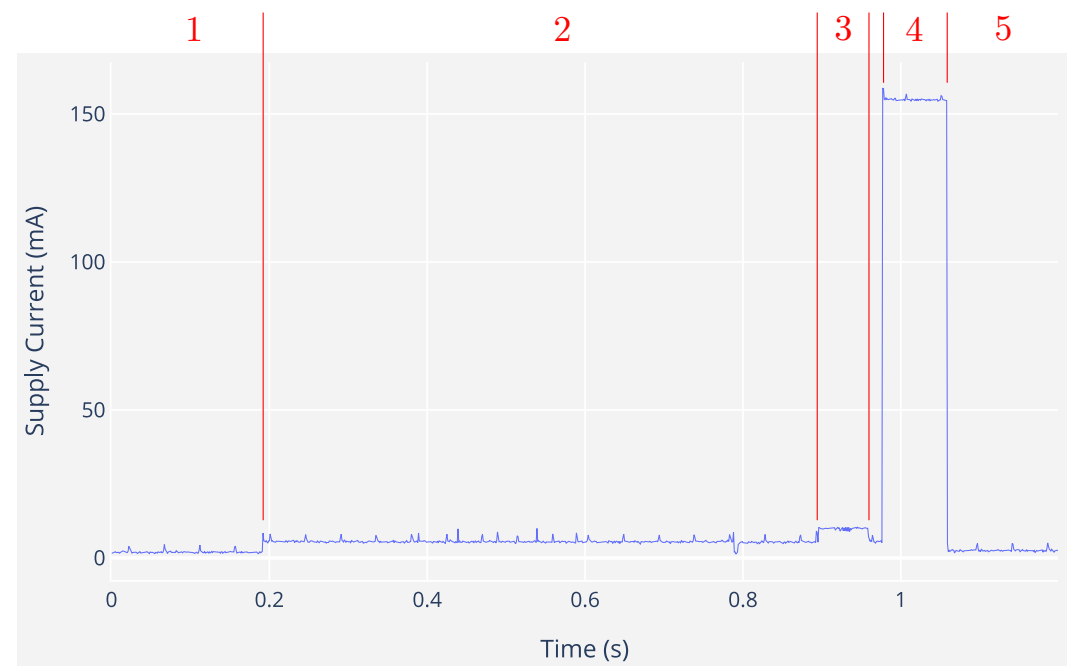

Figure 4.28: Current consumption profile for a single duty cycle of the SN. In region 1 and 5 the SN was idle. In region 2, the controller was collecting sensory data from all the sensors. In region 3, the radio was being activated. Finally, in region 4, the SN was transmitting a data packet.

\subsection{Summary}

In this chapter we explored the implementation of our designed system. We presented the results of its laboratory tests in addition to its field tests. The system's functionality and performance has been verified and established relative to the discussed application requirements. 


\section{Chapter 5}

\section{Entropic Sensing}

\subsection{Introduction}

The work presented in this chapter was inspired by the challenges highlighted in the

previous chapters. Specifically, the constrained energy budget of a wireless sensor. This work is intended for real-time monitoring applications where sensory data must be transmitted in real-time to inform time-critical control decisions.

As aforementioned in previous chapters, the use of WSNs to monitor industrial and environmental assets is on the rise. The ecosystem and the standards needed for the full adoption of WSNs is currently being shaped by the big players in the telecommunication sector. However, one crucial component in any WSN which will always be application dependent is the $\mathrm{SN}$.

More applications are surfacing recently, sparked by academic and commercial interest. Every application has requirements that originate from a unique set of operating conditions, performance goals, and design constraints. Since application requirements are the primary driver of hardware design, each application needs a custom-designed SN. The need for a unique SN in each application is also asserted 
by [11] after their review of the hardware specifications for over 40 different SNs.

While hardware design requirements and their associated challenges vary from one application to another, there is one persistent challenge in almost every design - the limited energy budget of wireless sensors. Even with the recent advances in energy storage and harvesting technology, it remains one of the primary restricting factors in any wireless sensing hardware design.

Energy budgets can be balanced by either increasing supply or decreasing demand. Increasing energy supply relates directly to the battery's capacity or the output power of a given harvesting technology. Both battery capacity and harvesting technologies are dependent on the application at hand. Generally, it is more difficult to address the energy challenge from the supply side. However, something can be done from the demand side that can reduce power consumption in potentially any SN. In this chapter, I will demonstrate my attempts to reduce the power consumption of SNs by reducing the number of data packets an SN has to transmit in real-time.

The SN is usually battery powered and is typically composed of four major functional blocks, the power supply, the controller, the sensor/transducer, and the wireless transceiver, as shown in Figure 2.2. The transceiver is an essential component in any SN. By definition, a wireless sensor must have a wireless transceiver onboard. In addition to our work in Chapter 4, authors of [109-111] concur that the transceiver is the most power-demanding component in the SN. Thus, by reducing the number of instances of wireless transmissions, we can effectively reduce the power consumption of SNs. 


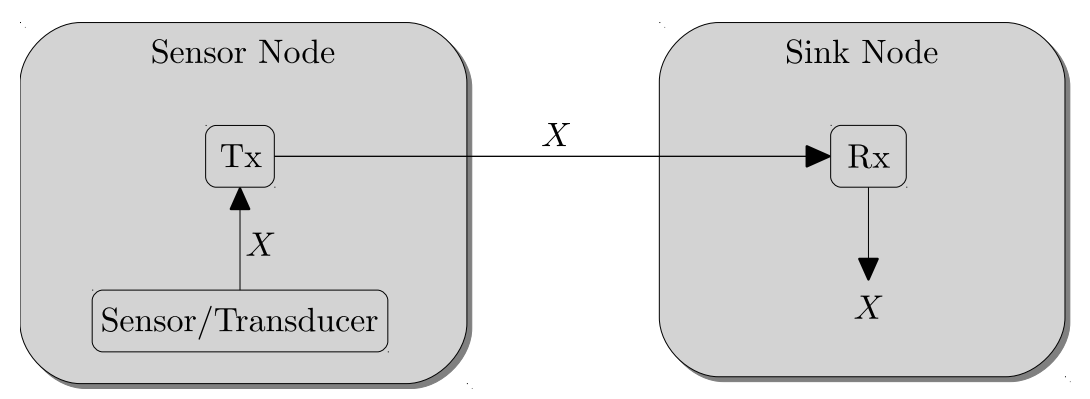

Figure 5.1: The signal path in a typical WSN.

In the context of monitoring applications where the WSN is deployed to monitor the condition or the status of an industrial asset, the SN takes a measurement and transmits the reading back to the sink instantaneously. The aim of this research is to maximize information transfer from the SN to the sink while minimizing data transfer. There are several approaches to this problem. Each approach puts forward its assumptions and has its limitations. We briefly discuss two well-known methodologies and then state our approach.

\subsubsection{Existing Approaches}

Data compression is one approach to reduce the payload of each data packet before transmission. By exploiting repetitions in the time-series a compression algorithm can reduce its size. The compressing code is designed based on the statistical distribution of the given time-series.

For example, in [112], a variable length Huffman code is used to compress temperature and humidity data on the SN. By assigning short code words to the smaller differences in subsequent measurements, a lossless compression ratio of $67 \%$ was achieved.

Data compression is an effective approach when the data payload is relatively large, such as when an SN is transmitting numerous sensor readings in a single 
packet. However, it is not the best approach to our application for two reasons. First, the energy savings from reducing the payload can be limited even with the $67 \%$ compression ratio put forth in [112] because of the overhead required by channel coding and by the network protocols for framing the data packet. Such overhead can depreciate power savings made by reducing the payload size. Also, activating the radio is power-consuming as shown in Figure 4.28. Second, in a data compression approach, the statistical distribution of the signal is usually known. This allows for designing the optimal code for the given signal [112]. Thus, for multiple sensors on board the SN, several codes will be implemented to compress the collected samples before transmission. Therefore, prior knowledge about the statistical structure of the sensory data would be needed to choose the code.

Some compression algorithms do not require prior statistical knowledge of the signal since they build their dictionary in real-time [113]. However, the given algorithms in [113] processes data in blocks of 528 bytes. This means, wireless transmission is triggered only after the block is full with encoded data. Thus, real-time monitoring applications will not benefit from this approach.

Another popular approach is compressed sensing [114] [115]. It allows for compressing data significantly by representing the time-series vector in a given orthonormal basis (e.g., Fourier, wavelet) in which the signal's transform coefficients are assumed to be sparse. The sparsity assumption holds for most natural signals and images [115], this is the reason for the success of transform coding compression [116]. Compressed sensing has numerous applications in many areas. For example, remote sensing [117], tomography [118], spectrum sensing [119], and RADAR [120].

Compressed sensing is built on convex optimization, which assumes the data is 
available in batches. Conversely, in our case, the data arrives as individual samples in a one-dimensional time-series. While there has been some work on dynamic compressed sensing [121] [122], the sparsity assumption is still essential in such works. That is, the measurements are taken incoherently from a signal whose representation in a given basis transformation contains a sparse set of coefficients.

Compressed sensing may not apply to our application for two reasons. First, sensory data in real-time monitoring applications do not arrive in batches. Second, the sparsity assumption cannot be made for any signal without prior knowledge about its representation in a given basis transformation.

Generally, in a WSN, the sink has significantly more energy and computing resources than an SN. This notion is adopted by researchers because it is inline with field experience and common engineering sense [123]. Thus, our approach will exploit this difference in energy supply and computing resources between the sink and an SN. By efficiently omitting information at the SN, we can reduce wireless traffic and, by extension, the SN's energy expenditure. Then, at the sink, we can afford to spend more energy and use computing resources to recover the omitted information in real-time.

We aim to reduce the number of samples transmitted by the SN and recover all the omitted samples without causing too much error in the reconstructed time-series. Our approach is based on the intuition that samples are not equally valuable in the information sense as some samples carry more information than others. 


\subsection{Entropy}

We need to find a metric to measure how a given sample contributes to the total complexity of a discrete time-series. This metric would allow the SN to rank samples as they are collected and decide on each one whether it is worth the energy expenditure for transmitting it to the sink or omit it from the real-time stream.

In the literature, there are plenty of metrics that can measure the complexity of a time-series. At a fundamental level, there is no agreement among researchers on the axioms and postulates of a complexity quantifier. I will go over some of the important metrics introduced and later will choose one of them for this work. The choice is based on two criteria:

1. The chosen metric should be computationally simple to be implemented on resource-limited hardware platforms (i.e., a microcontroller).

2. The chosen metric must be able to recognize samples of sudden events in the time-series to transmit them in real-time.

Being one of the fundamental concepts of information theory, the notion of information entropy [124] (as opposed to the thermodynamic entropy) was introduced originally by Shannon in 1948 [125]. Since then, the postulates and the axioms of this notion were debated among researchers. Consequently, other formulations of the entropy was crafted mainly to address the need for quantifying information and complexity of statistical data. Entropy can be expressed in nats or bits depending on the base of the logarithm in its formulation. Moving forward, the entropy in this work will always be expressed in bits (i.e., the logarithms are taken to the base 2).

The Shannon entropy of a discrete random variable is defined by equation 5.1. 
It is also called the self information, which is a measure of the average information content in one sample of $X$.

$$
H(X)=-\sum_{x_{n} \in \mathcal{A}} p\left(x_{n}\right) \log _{2} p\left(x_{n}\right)
$$

The Rényi entropy [126] expressed in equation 5.2 was introduced as a generalization to Shannon's entropy that satisfies the postulates made by Fadeev [127] to characterize the quantity $H(X)$. Rényi refers to $\alpha$ as the order of the distribution of $X$ where as $\alpha>0$ and $\alpha \neq 1$, however, as we approach the limit $\alpha \rightarrow 1$ the entropy $H(X)$ converges to Shannon's formula (equation 5.1)

$$
H_{\alpha}(X)=\frac{1}{1-\alpha} \log _{2}\left(\sum_{\mathcal{A}} p\left(x_{n}\right)^{\alpha}\right)
$$

To sum up, the Rényi family of absolute entropy measures include:

1. The the maximum (Hartley) entropy $H_{0}(X)$ which is the maximum possible entropy for a given alphabet $\mathcal{A}$. It is easy to derive it from Shannon's formula (equation 5.1) by imposing a uniform distribution on $X$. This means that the maximum entropy of $X$ (i.e., the most uncertainty) is obtained when all of its states are equally probable at every time step. That is to say we have the least amount of information about $X$ because its past did not favour any of its states over another in the number of occurrences. The maximum entropy $H_{0}(X)$ is simply defined by the base-two logarithm of the cardinality of $X$ 's alphabet as shown in equation 5.3.

$$
H_{0}(X)=\log _{2} \# \mathcal{A}
$$

2. Shannon entropy $H_{1}(X) \equiv \lim _{\alpha \rightarrow 1} H_{\alpha}(X)$ 
3. Collision entropy $H_{2}(X) \equiv \lim _{\alpha \rightarrow 2} H_{\alpha}(X)$

4. The minimum entropy $H_{\infty}(X) \equiv \lim _{\alpha \rightarrow \infty} H_{\alpha}(X)$

Rényi's generalization was still not satisfying all researchers' needs. Furthermore, with a widespread disagreement on the axioms of an essential information theoretic quantity such as the entropy, one can only go back to the most fundamental discussions of this concept in hopes of a more unified understanding and formulation. Consequently, in a well put discussion on the axioms of entropy and sparsity $[128,129]$ we have a generalization to many of their measures including Rényi's.

Aside from absolute entropy measures, it is worth mentioning that Rényi's work [130] also included a generalization for the relative entropy (Kullback-Leibler divergence [124]) between two probability mass functions.

Moment statistics (e.g., mean, variance) has a holistic approach to characterizing time-series. This also applies to the entropy in its traditional sense. Other entropy measures were created to capture the predictability/regularity and the complexity of time-series. Pincus introduced approximate entropy (ApEn) [131] in an attempt to adapt the entropy measures of Kolmogrorov, Sinai, and Oseledets [131] to practical (limited in size) data sets. ApEn is measure of complexity that applications in physiology [132], finance [133], and climatology [134].

Later on the sample entropy (SampEn) was introduced as an improvement on ApEn. Unlike ApEn, the SampEn decouples the size of the time-series from the calculation. Also, SampEn eliminates the bias in ApEn resulting from self counting. Along with other advantages, SampEn seems to be a better formulation for ApEn [135]. 
Multiscale entropy (MSE) [136] builds on SampEn and ApEn and includes multiple time scales of measurement. This allows MSE to assess the complexity of a given time-series at longer and shorter time scales. Also, MSE quantifies complexity as the sum of entropies over all time scales. Much like ApEn and SampEn, MSE is also applied in biological signal analysis [137].

Permutation entropy [138] is another attempt at quantifying the complexity of time-series using a simple algorithm that can work with practical data sets. Although permutation entropy can, in some cases, give false conclusions about the temporal structure of the time-series [139], it is still a valuable step in the right direction.

Let us now set aside this multitude of entropy measures and formulations. Quantifying the contribution of a single sample to the total information content or complexity of a time-series may require a whole separate study. Starting from the axioms just as in $[128,129]$, one may find a proper formulation for such quantity. The question remains whether such quantity can be easily computed on SN hardware. Furthermore, the goal of this work is to reduce the energy consumption of SN by omitting some samples from the real-time transmitted data. Thus, we will settle for the most basic formulation of the entropy, that is Shannon's entropy, in an attempt to demonstrate the concept of entropic sensing as a system rather than dive into an abstract study of one of the most fundamental concepts of information theory.

Hereinafter, whenever the term entropy is used it will refer exclusively to Shannon's entropy in equation 5.1. In the next section, I will demonstrate how Shannon's entropy can be used to capture the statistical and temporal structure of $X$ using less number of samples while ensuring real-time transmission of unpredictable events in $X$ all while bearing a reasonable computational load on the SN's hardware resources. 


\subsection{Formulation}

We assume knowledge of each individual sample in real-time in addition to some indicators of its past. Each sample, when captured by the SN, is judged to determine whether it should be transmitted or omitted based on the amount of information it adds to the total information content of the whole signal.

Let us consider the SN used in a monitoring application (say for GET) where a certain physical quantity (e.g., temperature, pressure, proximity) is being measured regularly (with equal time delay between measurements) and measurements (later referred to as samples) are transmitted in real-time to a sink over a half-duplex channel. The sensory data stream forms a discrete time-series. Each measurement (sample) is transmitted in real-time in a single data packet.

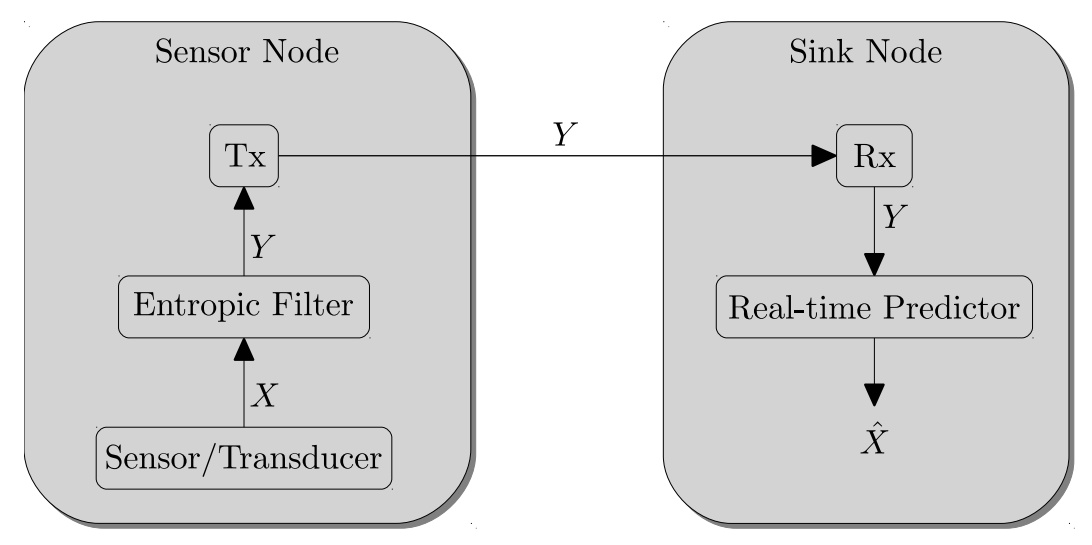

Figure 5.2: The signal path in the introduced entropic sensing system.

The objective is to reduce the energy consumption at the $\mathrm{SN}$ by minimizing the number of transmitted samples while keeping error in the reconstructed $X$ (later referred to as $\hat{X}$ ) as small as possible. This means the SN will not transmit a subset of $X$ samples as they are collected in real-time. The important question is how can 
the SN determine which samples get to be transmitted to the sink and which ones will be omitted?

$X$ is a discrete time-series to be transmitted from an SN to a sink node. $Y$ is the observed r.v. at the receiver which will be used to construct and estimate of $X$ called

$\hat{X}$. The solution then is to find the best estimate $\hat{X}$ for the current instance of $X$ given the current and the past instances of $Y$ and $\hat{X}$.

The sensor readings form a discrete time-series $X$ with an alphabet $\mathcal{A}$ and a probability mass function $(\mathrm{PMF}) \operatorname{P}\left(x_{n}\right)=\operatorname{Pr}\left\{X=x_{n}\right\}$ where $x_{n} \in \mathcal{A}$ such that $n \in\{1,2,3, \ldots N\}$ where $N=\# \mathcal{A}$ (i.e., $N$ is the cardinality of the set $\mathcal{A}$ ). In other words, $N$ is the number of bins in the histogram of $X$. Furthermore, $X$ produces uniform time samples represented by the vector $S=\left\{s_{1}, s_{2}, \ldots, s_{t}, \ldots, s_{T}\right\}$, where $T$ is the total number of samples collected from $X$.

\subsubsection{Assumptions}

The following assumptions are made to set the framework of simulations and to outline the suitability of entropic sensing for different applications.

1. The probability of error in $Y$ is zero. This assumption is made to simplify simulations. The point of this work is to demonstrate entropic sensing as a methodology. Thus, introducing channel imperfections will not put the proposed approach at any advantage or disadvantage compared to the existing methods because all are subject to the limitations of wireless channels. In fact, one may argue that entropic sensing reduces wireless traffic and therefore might be less prone to channel induced errors such as packet collisions.

2. $X$ is a regularly sampled discrete time-series. In other words, time intervals 
between $X$ 's samples are equal. Also, $X$ 's samples are quantized to a finite predefined set of bins.

3. The SN has only one transducer/sensor onboard. This is a restricting assumption that can hinder the applicability of this work pending further research work. Discussed in section 5.7.

\subsubsection{Generalizations}

We can generalize the applicability of entropic sensing with these points. Each point represent a liberating assumption that puts this method at an advantage when compared with other methods lacking such generalizations.

1. There is no prior knowledge about $X$. Unlike what is assumed in compressed sensing schemes [140] we assume no prior knowledge about the compressing basis or the statistical structure of $X$. Thus, it is not possible to construct an optimal code for $X$. This brings ES closer to practical implementation.

2. The $S N$ does not need a firmware update over the air. While having the option to update the firmware during operation can improve and further tune our algorithms, however, most commercially available chips still lack this capability. Assuming no firmware updates mid-operation brings this work closer to practical implementation.

3. Communication is unidirectional from the $S N$ to the sink. In other words, there is no need for a full-duplex channel. This widens the applicability scope of this approach and adds to its spectral efficiency. 


\subsubsection{Benchmarking Time-Series}

In this section, we go over the set of discrete time-series or signals chosen to evaluate the performance of the given entropic sensing system. All signals were normalized before being processed. The normalization was done to facilitate comparing performance between all signals, however, in a real implementation, no normalization is required.

1. A constant signal with a square pulse. This signal was introduced to make sure that samples representing significant unpredictable events in the signal will be picked up by the glsEF algorithm and transmitted in real-time.

$$
S 1= \begin{cases}1 & 0.5 T \leq t \leq 0.6 T \\ 0 & \text { Otherwise }\end{cases}
$$

2. A linear monotonic signal increasing in time with a constant slope. This signal is intended to evaluate the predictor's performance in handling an easily predictable signal.

$$
S 2=1.2 t
$$

3. A simple sinusoidal signal with a single frequency and no added noise. This and the next three signals represent a sinusoidal waveform with a staggered increase in complexity. $S 3$ is the simplest form.

$$
S 3=A \sin (t)
$$

where $A=10$ is the amplitude. 
4. We add four equal-amplitude harmonics to the previous signal to get $S 4$. No added noise.

$$
S 4=A \sin \left(\frac{t}{4}\right)+A \sin \left(\frac{t}{2}\right)+A \sin (t)+A \sin (5 t)
$$

5. Now we add noise to $S 4$ to increase the complexity of $S 5$. We use additive white Gaussian noise (AWGN).

$$
S 5=A \sin \left(\frac{t}{4}\right)+A \sin \left(\frac{t}{2}\right)+A \sin (t)+A \sin (5 t)+A_{n} N(\mu=0, \sigma=0.8)
$$

where $A_{n}=5$ is the noise amplitude.

6. Finally, we introduce an unpredictable event similar to $S 1$ in order to evaluate the system's performance in picking up such events in real-time.

$$
S 6= \begin{cases}S 5+10 A & 0.5 T \leq t \leq 0.6 T \\ S 5 & \text { Otherwise }\end{cases}
$$

7. $S 7$ is a non-stationary signal from an iterative generator and AWGN as follows. Since, most physical signals are non-stationary, it will be interesting to explore the system's performance in handling such signals.

$$
s_{t}=s_{t-1}+A_{n} N(\mu=0, \sigma=0.8) \quad \text { for } t=\{2,3,4, \ldots, T\}
$$

where, $s_{1}=\operatorname{random}(-1,1)$ and $A_{n}=20$, such that $\operatorname{random}(x, y)$ is a random generator with a uniformly distributed output of two states ( $x$ and $y$ ). 
8. To stretch our system to its limits, let us introduce a purely random signal. $S 8$ is a random walk produced with an iterative generator as follows,

$$
s_{t}=s_{t-1}+\operatorname{random}(-1,1) \quad \text { for } t=\{2,3,4, \ldots, T\}
$$

where, $s_{1}=\operatorname{random}(-1,1)$.

9. Now for comparison, we use series that are commonly used in literature. $S 9$ and $S 11$ are two of the most commonly used series in time-series analysis and prediction research. $S 9$ is the Santa Fe laser time-series [141,142].

10. $S 10$ is called the Hénon map [143]. It is one of the widely studied discrete-time dynamic system with chaotic output [142]. This is used to evaluate the system's performance in chaotic signals which are common in the physical world. $S 10$ is generated as follows,

$$
s_{t}=1-a s_{t-1}^{2}+b s_{t-2} \quad \text { for } t=\{3,4, \ldots, T\}
$$

where $s_{1}=s_{2}=0, a=1.1$, and $b=0.3$.

11. $S 11$ is the Leuven time-series [141].

\subsection{Entropic Filter}

This section introduces the Entropic Filter (EF) algorithm which is the heart of the entropic sensing system.

At the very beginning, the SN will attempt to prime the predictor with basic knowledge about the temporal and statistical structure of $X$. This means the SN 
will transmit a preset number of priming samples (nps) directly to the sink without filtering. Afterwards, the EF algorithm will be engaged till the end.

As time progresses, samples of $X$ arrive consecutively. At each time step $t$ the newly added sample will alter the distribution of $X$ and in turn its entropy $H(X)$. In other words, $H(X)$ changes every time a new sample is added to the series $X$. This change in $H(X)$ is what the $\mathrm{SN}$ will exploit in making the transmit/omit decision for each sample.

Now that we have established that $H(X)$ is constantly changing with time $t$. Let us reserve the notation $H(X)$ for the total entropy of $X$ and define instantaneous entropy (IE) of $X$ as follows.

Definition The instantaneous entropy (IE) of $X$ at time $t$ denoted by $H(X)_{t}$ is the entropy of $X$ computed from the sample set $S=\{1,2,3, \ldots, t\}$.

As a new sample $\left(s_{t}\right)$ arrives, the new value of the entropy is computed. It is computationally demanding to use Shannon's formula in equation 5.1 which requires the re-computation of $X$ 's histogram every time step. Fortunately, there is a more efficient method of computing the IE $H(X)_{t}$ from its own previous value $H(X)_{t}-1$ and a few other easily computed terms. This method is derived and discussed in section 5.4.1.

Figure 5.3 shows the flow chart for the glsEF algorithm. It consists of two parallel branches. Both branches are identical except that the right branch operates on the first difference of $X$. 


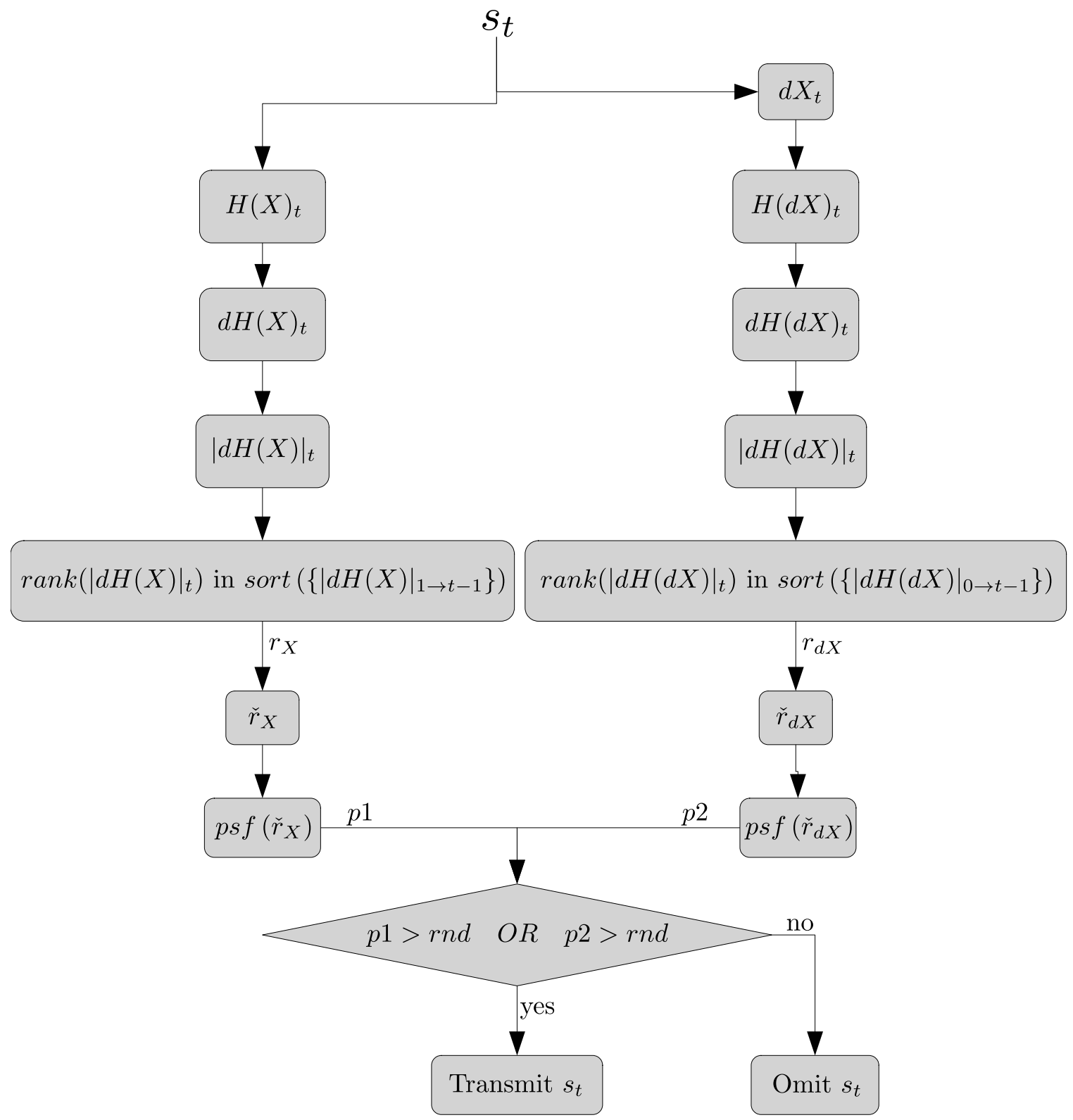

Figure 5.3: The flow chart for entropic filtering.

Analogous to the difference quotient in single-variable calculus, let us define the "difference of the instantaneous entropy" of $X$ at time $t$ (denoted by $\left.d H(X)_{t}\right)$ as the change in the IE $H(X)_{t}$ due to the addition of the most recent sample $s_{t}$ to $X$. 
Definition For a discrete time-series $X$ with uniform time samples represented by the vector $S=\left\{s_{1}, s_{2}, \ldots, s_{t}, \ldots, s_{T}\right\}$, the IE difference at time $t$ is the difference between the IE of $X$ at time $t$ and that at time $t-1$. denoted by $d H(X)$. The IE difference is a vector with size $T-1$.

$$
d H(X)_{t}=H(X)_{t}-H(X)_{t-1} \quad \forall \quad t \in\{2,3, \ldots T\}
$$

After calculating the instantaneous entropy difference, we get its absolute value as shown in Figure 5.3. This step is introduced because a sample that reduces $H(X)_{t}$ significantly is equally important to a sample that increases $H(X)_{t}$ significantly.

Then we come to the point where we compare $|d H(X)|_{t}$ to its previous values and rank its value in a sorted vector of all the previously stored values. The rank of $\left|d H(X)_{t}\right|$ will be a number $r$ indicating the position where $|d H(X)|_{t}$ should be inserted if we where to keep $|d H(X)|_{1 \rightarrow t-1}$ sorted. Keep in mind that $r \in\{1,2,3, \ldots, t\}$ and before passing it to the Probability Shaping Function (PSF) denoted by psf it is divided by $t$ to normalize it. The normalized rank is denoted by $\check{r}$ and the $p s f$ is discussed in details in section 5.4.2.

The steps of entropic filtering $S 6$ are shown in Figure 5.4. Similar plots for all the other benchmarking signals are in Appendix B.1. 

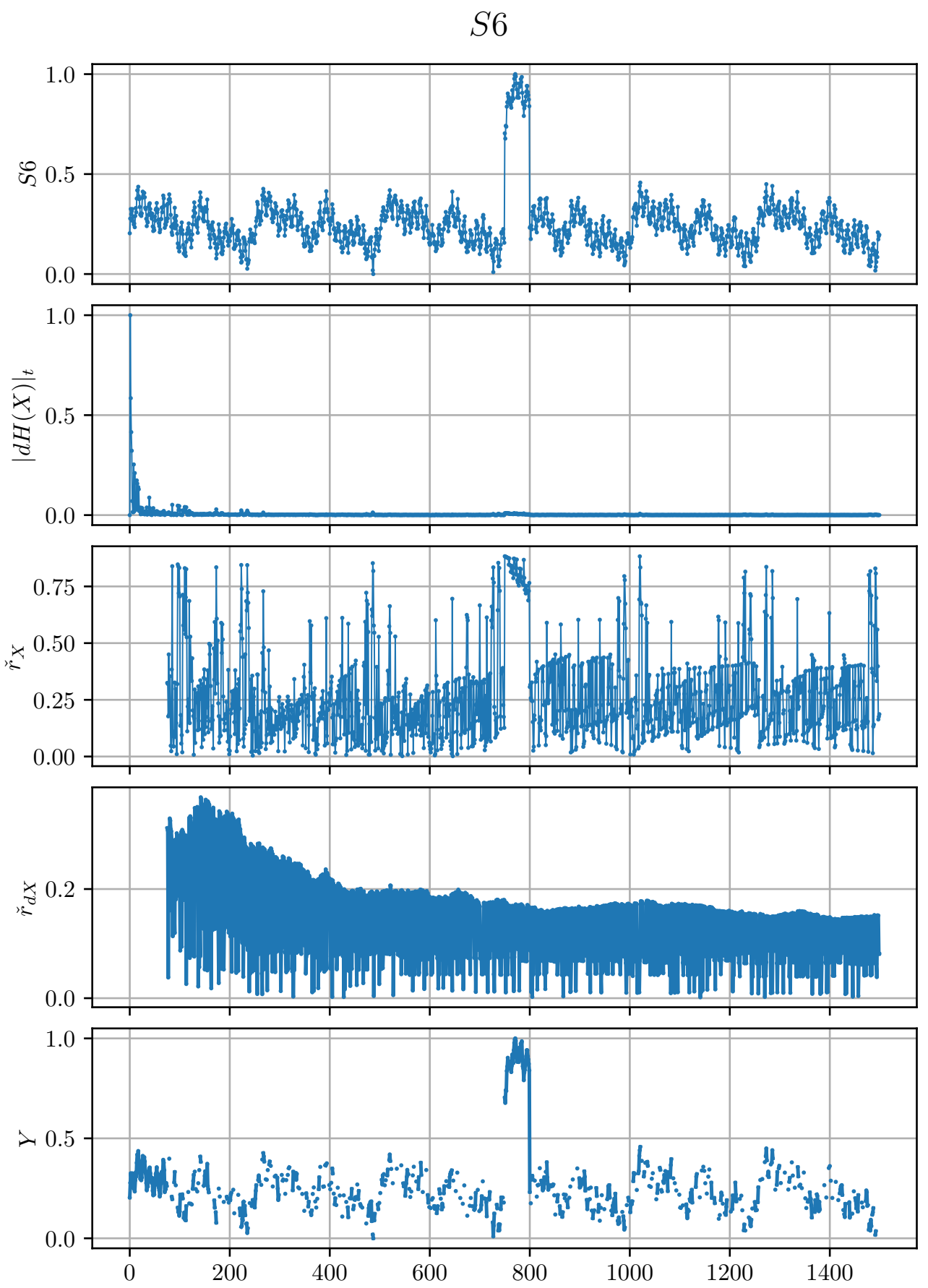

Figure 5.4: Entropic filtering steps for $S 6$. 


\subsubsection{Computing Entropy}

Computing entropy is to be performed every time step on resource limited SN's hardware. Thus, it is essential that we seize every opportunity to compute $H(X)_{t}$ efficiently at every time step.

Traditionally, we would start by empirically calculating the PMF of $X$ (i.e., the histogram $\left.h i s t(X)_{t}\right)$ from all its previously collected samples. Then, we use equation 5.1 to calculate the entropy.

Looking into the traditional method reveals considerable redundancies in calculating histograms and the entropy summation repeatedly at every time step. Not to mention the need to store all previous samples of $X$ locally on the SN throughout its lifetime. Consequently, I constructed an iterative computing method for $H(X)_{t}$ in a more efficient way from $H(X)_{t-1}$ and $\operatorname{hist}(X)_{t-1}$.

The entropy at $t-1$ and at $t$ as shown in equations 5.14 and 5.15 .

$$
\begin{gathered}
H(X)_{t}=-\sum_{x_{n} \in \mathcal{A}} p\left(x_{n}\right)_{t} \log _{2} p\left(x_{n}\right)_{t} \\
H(X)_{t-1}=-\sum_{x_{n} \in \mathcal{A}} p\left(x_{n}\right)_{t-1} \log _{2} p\left(x_{n}\right)_{t-1}
\end{gathered}
$$

Note that $p\left(x_{n}\right)$ is indexed by $t$ for the same reason $\operatorname{hist}(X)$ is also indexed by $t$ and that is because the histogram of $X$ changes at every time step by the addition of $s_{t}$. This means that we have to keep updating hist $(X)_{t}$ as time progresses. Fortunately, we do not have to compute $\operatorname{hist}(X)_{t}$ every time from the whole population of samples.

When we progress through time $s_{t}$ is captured at every time step, and it will be enlisted to only one of the states in $\mathcal{A}$ denoted by $x_{r t}$. This means that the probability 
of the most recent state (i.e., at the current time) $p\left(x_{r_{t}}\right)_{t}$ will increase from its previous value $p\left(x_{r_{t}}\right)_{t-1}$. On the other hand, the probability of all the other states will decrease by a factor of $\frac{t-1}{t}$. To explain the notation of $p\left(x_{r_{t}}\right)_{t}$ further, the letter $r$ indicates the most recent state, $x_{r_{t}}$ is the state of $s_{t}$ where $r_{t} \in\{1,2,3, \ldots N\}$, and the probability of this particular state $x_{r_{t}}$ at time $t$ is denoted as $p\left(x_{r_{t}}\right)_{t}$. Similarly, the state of $s_{t-1}$ is $x_{r_{t-1}}$ and its probability at time $t-1$ and $t$ is $p\left(x_{r_{t-1}}\right)_{t-1}$ and $p\left(x_{r_{t-1}}\right)_{t}$ respectively.

Now, let us define $\mathcal{A}_{t}^{\prime}$ as the set of all states of $X$ except the state in which $s_{t}$ is enlisted. Thus,

$$
\mathcal{A}_{t}^{\prime}=\mathcal{A} \backslash\left\{x_{r_{t}}\right\}
$$

Now let us see how the probabilities in $\operatorname{hist}(X)_{t}$ can be updated from $\operatorname{hist}(X)_{t-1}$.

$$
p\left(x_{n}\right)_{t}=\frac{t-1}{t} p\left(x_{n}\right)_{t-1} \quad \text { where } x_{n} \in \mathcal{A}^{\prime}
$$

and,

$$
p\left(x_{r_{t}}\right)_{t}=\frac{t-1}{t} p\left(x_{r_{t}}\right)_{t-1}+\frac{1}{t}
$$

The time index $t$ is used for the recent state corresponding to $s_{t}$, and its probability. The time index is also used for its numerical value to represent the total number of samples collected up to the current time $t$.

Define

$$
H(C X)=-\sum_{x_{n} \in \mathcal{A}} C p\left(x_{n}\right) \log _{2}\left(C p\left(x_{n}\right)\right) \quad \text { where } 0 \leq C \leq 1
$$

Then, it follows that, 


$$
\begin{gathered}
H(C X)=-\sum_{x_{n} \in \mathcal{A}} C p\left(x_{n}\right) \log _{2} C+C p\left(x_{n}\right) \log _{2} p\left(x_{n}\right) \\
H(C X)=-C \log _{2} C \underbrace{\sum_{x_{n} \in \mathcal{A}} p\left(x_{n}\right)}_{=1}-C \underbrace{\sum_{x_{n} \in \mathcal{A}} p\left(x_{n}\right) \log _{2} p\left(x_{n}\right)}_{=H(X)}
\end{gathered}
$$

Therefore,

$$
H(C X)=-C \log _{2} C+C H(X)
$$

Now, let us extract the term corresponding to the most recent state $x_{r_{t}}$ from the summation in equation 5.14. hence, we get,

$$
H(X)_{t}=-p\left(x_{r_{t}}\right)_{t} \log _{2} p\left(x_{r_{t}}\right)_{t}-\sum_{x_{n} \in \mathcal{A}^{\prime} t} p\left(x_{n}\right)_{t} \log _{2} p\left(x_{n}\right)_{t}
$$

By substituting equations 5.17 and 5.18 in 5.23 we get:

$$
\begin{aligned}
H(X)_{t}= & \overbrace{-\left(\frac{t-1}{t} p\left(x_{r_{t}}\right)_{t-1}+\frac{1}{t}\right) \log _{2}\left(\frac{t-1}{t} p\left(x_{r_{t}}\right)_{t-1}+\frac{1}{t}\right)}^{\text {part1 }} \\
& \overbrace{-\sum_{x_{n} \in \mathcal{A}^{\prime} t}\left(\frac{t-1}{t} p\left(x_{n}\right)_{t-1}\right) \log _{2}\left(\frac{t-1}{t} p\left(x_{n}\right)_{t-1}\right)}^{\text {part } 2}
\end{aligned}
$$

for simplicity let $\frac{t-1}{t}=C$ and $p\left(x_{r_{t}}\right)_{t-1}=R$. Then, part 1 becomes,

$$
\text { part } 1=-\left(C R+\frac{1}{t}\right) \log _{2}\left(C R+\frac{1}{t}\right)
$$


and part2 becomes,

$$
\text { part } 2=-\sum_{x_{n} \in \mathcal{A}^{\prime} t} C p\left(x_{n}\right)_{t-1} \log _{2}\left(C p\left(x_{n}\right)_{t-1}\right)
$$

From equation 5.23 we get,

$$
\operatorname{part2}=C p\left(x_{r_{t}}\right)_{t-1} \log _{2}\left(C p\left(x_{r_{t}}\right)_{t-1}\right) \underbrace{-\sum_{x_{n} \in \mathcal{A}} C p\left(x_{n}\right)_{t-1} \log _{2}\left(C p\left(x_{n}\right)_{t-1}\right)}_{=H(C X) \text { from equation } 5.19}
$$

Then, from equation 5.22 we get,

$$
\text { part2 }=-C \log _{2} C+C H(X)_{t-1}+C p\left(x_{r_{t}}\right)_{t-1} \log _{2}\left(C p\left(x_{r_{t}}\right)_{t-1}\right)
$$

Recalling part 1 from equation 5.25, part2 from equation 5.28, and recombining them in equation 5.24 we finally get,

$$
\begin{array}{r}
H(X)_{t}=-\left(C R+\frac{1}{t}\right) \log _{2}\left(C R+\frac{1}{t}\right)-C \log _{2} C+C H(X)_{t-1} \\
+C p\left(x_{r_{t}}\right)_{t-1} \log _{2}\left(C p\left(x_{r_{t}}\right)_{t-1}\right)
\end{array}
$$

To express equation 5.29 in a simpler form, let us define a function $f(x)=x \log _{2} x$ with following the convention $0 \log _{2} 0=0$ based on the argument of continuity as in [124]. Therefore equation 5.29 can be rewritten as,

$$
H(X)_{t}=-f\left(C R+\frac{1}{t}\right)-f(C)+C H(X)_{t-1}+f(C R)
$$


As we can see in equation 5.30, the entropy at time $t$ can be efficiently calculated from its own value at time $t-1$. To compare, let us rewrite Shannon's formula by using $f(x)=x \log _{2} x$ as follows.

$$
H(X)_{t}=-\sum_{x_{n} \in \mathcal{A}} f\left(p\left(x_{n}\right)_{t}\right)
$$

Thus, using Shannon's formula entails $N$ computations for $f(x)$, and $N-1$ summations while using the iterative formula in equation 5.30 entails only three computations for $f(x)$, five summations and three multiplications. Additionally, the iterative formula is not a function of $N$ which means we can increase the resolution of a sensor's output without worrying about an extra computing cost for the SN.

In Figure 5.5, $H(X)_{t}$ is computed by Shannon's formula and again with the iterative formula to confirm that both produces the exact same values. The same plot for all the benchmarking signals are listed in Appendix B.2. 

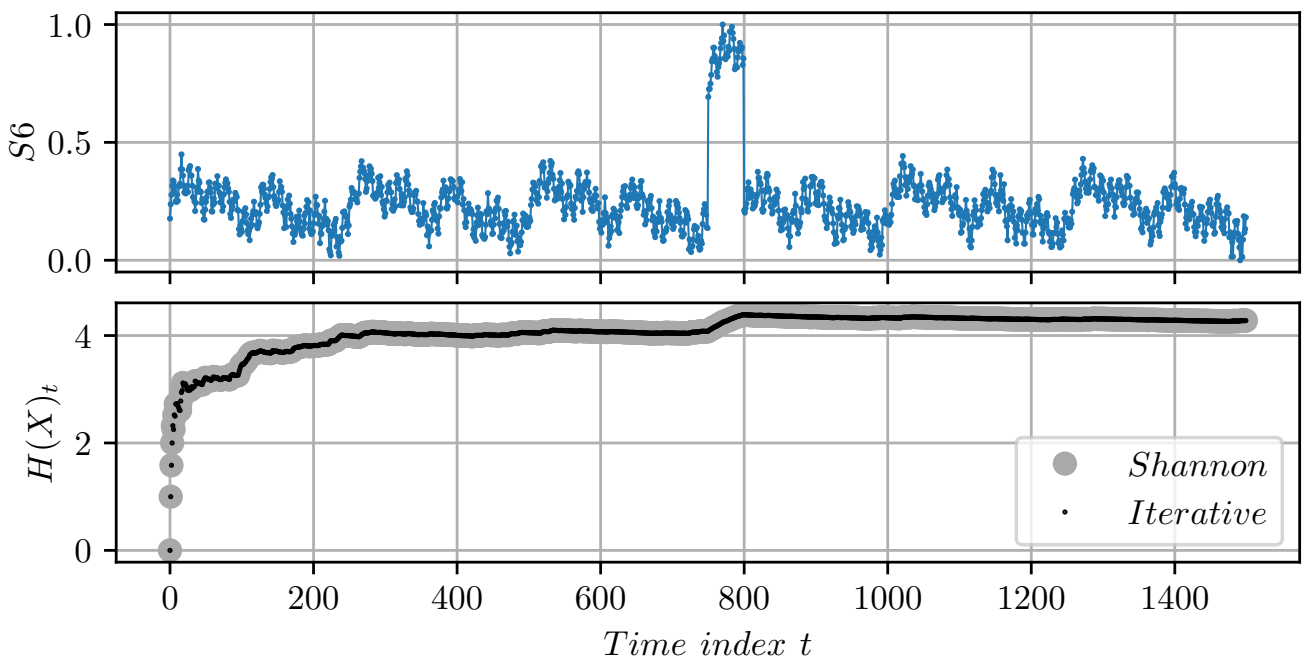

Figure 5.5: The discrete time-series and its IE using Shannon and the iterative formulas.

\subsubsection{Probability Shaping Function}

The PSF is a mapping function that allows for tuning the glsEF algorithm to favour samples from a given range of values for their entropy difference. This means we can design the PSF to favour low entropy samples, high entropy samples, or any combination that can improve the performance of our system for a given signal.

The PSF takes the normalized rank $\check{r}=\frac{r}{t}$ of the current sample and outputs the probability of transmitting it $p=p s f(\check{r})$.

In this work we have experimented with four different PSFs. We cannot claim that any of the four functions is optimized for a certain signal but, from the simulation results, there are clear advantages of using some over the others.

The four PSFs are:

1. psf 1 (high entropy): this function transmits (with probability one) high entropy 
samples whose $\check{r}$ is greater than or equal a predefined threshold $t h=0.7$. Sample whose $\check{r}$ is less than the threshold will be transmitted with probability equals to $\check{r}$ itself. The intuition behind this psf can be derived from the definition of entropy itself where high entropy samples are those which introduces new structure to the series and are harder to predict by the sink thus are more worthy of being transmitted. This intuition evident in other works [144].

$$
p= \begin{cases}\check{r} & \check{r}<t h \\ 1 & \check{r} \geq t h\end{cases}
$$

2. psf2 (low entropy): this function is the inverse of the high entropy $p s f$. It is included only to establish the proper distinction in the performance of the entropic sensing system between favouring low entropy samples vs high entropy samples. The threshold in this case is 0.3 .

$$
p= \begin{cases}1-\check{r} & \check{r}>t h \\ 1 & \check{r} \leq t h\end{cases}
$$

3. psf3 (mirrored sigmoids): this function consists of two sigmoid functions back to back. The intuition behind it is to include very low and high entropy samples in the transmission while omitting ones in the mid-low range. This $p s f$ is defined as follows,

$$
p=\frac{1}{1+e^{-(40 \check{r}-25)}}+\frac{1}{1+e^{(80 \check{r}-6)}}
$$

4. psf4 (high \& random): this psf combines the high entropy function above the 

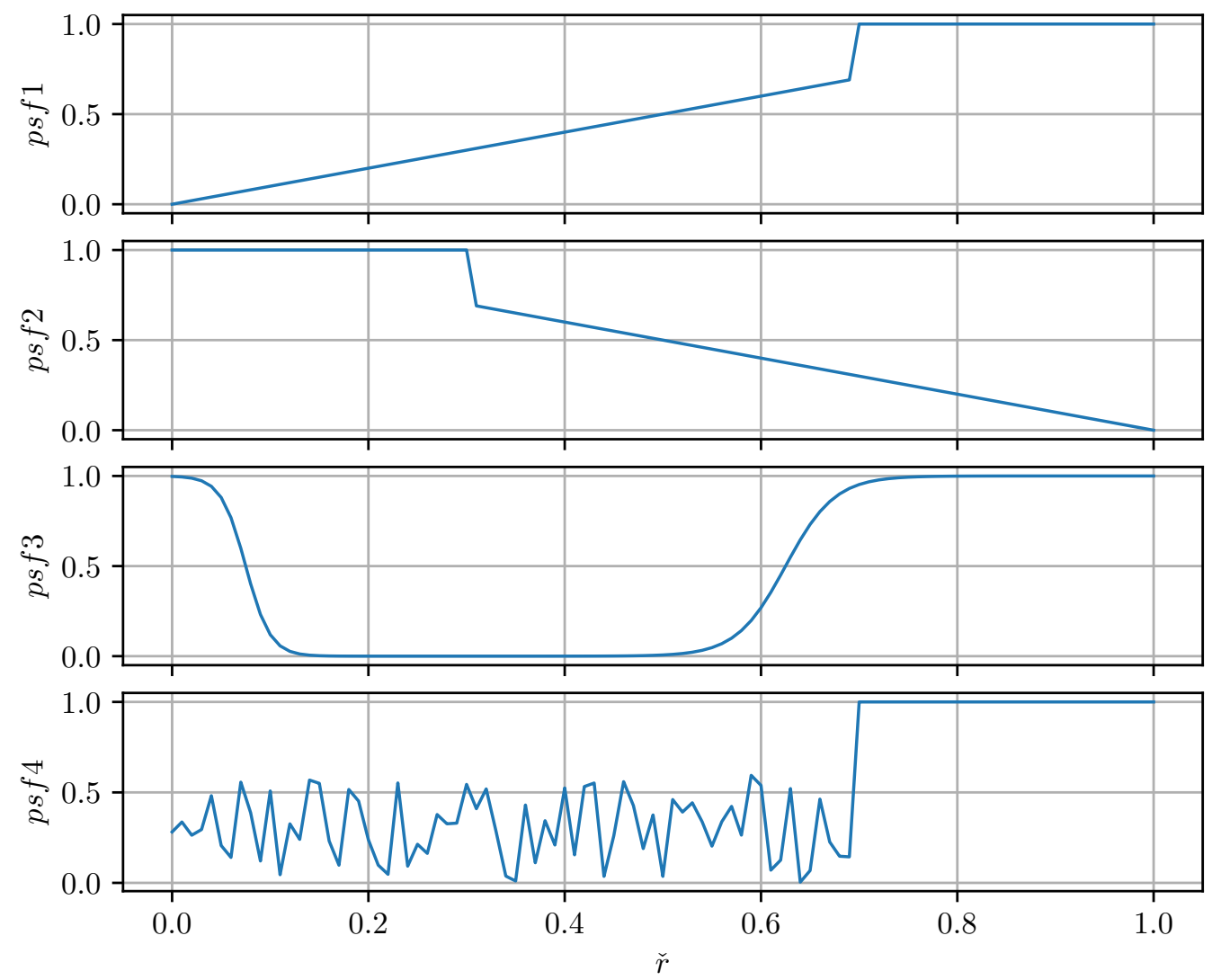

Figure 5.6: The four probability shaping functions used in this work.

predefined threshold th with a uniformly distributed random function below it. It is defined as follows,

$$
p= \begin{cases}\operatorname{random}(0 \rightarrow 0.6) & \check{r}<t h \\ 1 & \check{r} \geq t h\end{cases}
$$

To get a better sense of what the PSF does to the population of samples, I plotted the probability density function (PDF) of the normalized rankings $\check{r}_{X}$ and $\check{r}_{d X}$ of all 


\section{S5}

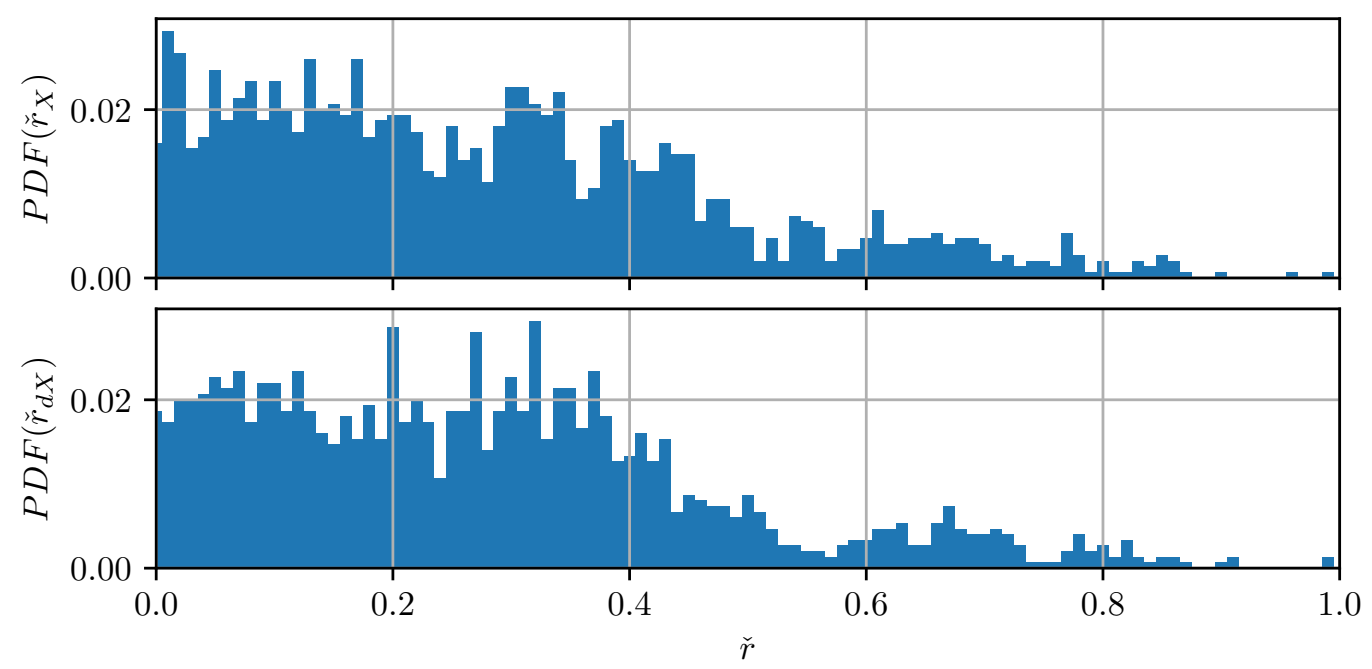

Figure 5.7: Histogram of $\check{r}$ for $X$ and $d X$ of $S 5$.

samples for $S 5$ as shown in 5.7. We can clearly notice the skewed distribution of $\check{r}$. Most samples have low entropy while few have high entropy. It important to transmit those high entropy samples and that is why three of our PSFs have a value of one for $\check{r}>0.7$. The PDF plots for the rest of our benchmarking series (S1 to S11) are included in Appendix B.3.

A note regarding the demonstrated histograms is that they are constructed by comparing each sample to the past samples only. One might think that if the comparison was made among all the samples (past and future), the histogram might be different. Nevertheless, in real-time we only have access to the past. Thus, it is not meaningful to construct any PSF by looking at a histogram that can never be reproduced in reality. 


\subsection{Recovery}

Entropic filtering leaves the signal with a significant number of omitted samples. Recovering the omitted samples at the sink is the responsibility of the predictor in Figure 5.2.

The flow chart for the predictor's operation is shown in Figure 5.8. It is repeated every time step. Thus it is important to choose a suitable predictor, taking into account the available hardware resources at the sink, because real-time execution must be attained.

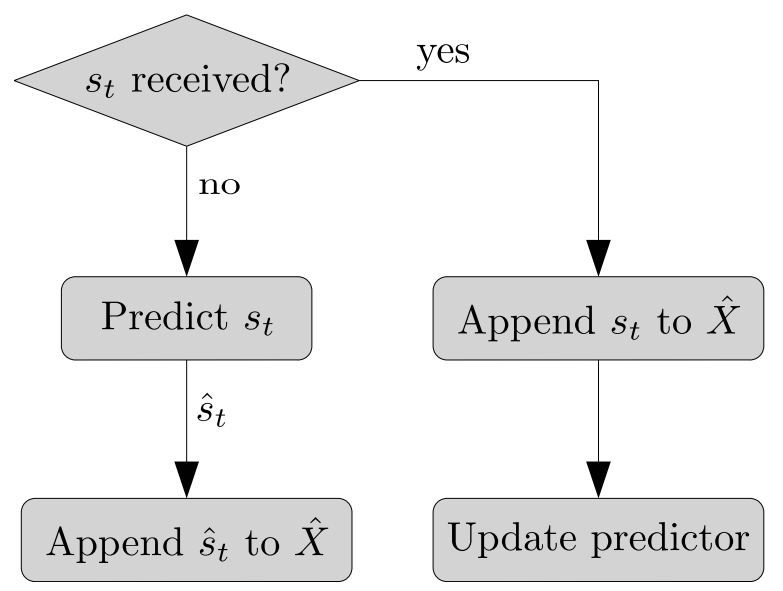

Figure 5.8: Flow chart of the predictor's operation inside the sink.

Every time step, the cycle starts with a condition. If $s_{t}$ is received, it is appended directly to $\hat{X}$. Then, the predictor goes through an iteration of updating its model by utilizing the recent information contained in $s_{t}$ as well as all the previous samples (received and estimated). On the contrary, if $s_{t}$ was not received at its designated time, the predictor tries to recover $s_{t}$ by predicting it based on its updated model. The predictor produces its estimate $\hat{s}_{t}$ and appends it to $\hat{X}$.

It is well known that the accuracy of predictions, by any given predictor, decreases 132 
significantly as the number of steps ahead is increased. Fortunately we do not have to be concerned about this because the probabilistic nature of the glsEF algorithm limits the number of consecutive omitted samples to a small value $(\approx 3)$.

\subsection{Evaluation}

We use simulation to evaluate the performance of the system. The simulation was coded in Python with the help of several open source libraries including Numpy, Pandas and Tensorflow. The full run of all the 11 signals using all four PSFs takes about 24 hours on a typical desktop computer without invoking parallelism in the code.

We use a deep-learning predictor because it can adapt to any signal on the fly. Better results may be possible if we optimize the choice of the predictor with an anticipated signal dynamics. For example auto-regressive integrated moving average (ARIMA) models could be faster and more accurate for some signals [145] but, when it comes to seasonal and/or non-stationary ones, ARIMA models require manual tuning, and that is far from ideal in real-time applications.

Recurrent Neural Network (RNN) in general and Long short-term memory (LSTM) networks in specific are known for various applications including nonlinear system identification [146], time-series prediction [147,148], and speech recognition [149]. Furthermore, RNN nodes form a directed graph along a temporal sequence which makes them able to capture temporal and statistical complexities of time-series. Thus, the chosen predictor is an LSTM network which had the same hyper-parameters through all simulations demonstrating its versatility.

For evaluating energy savings, we calculate the ratio between the cardinality of 
$Y$ that is the number of transmitted samples, and the cardinality of $X$, which is the number of captured samples and is equal to $T$. Let us call it the samples reduction ratio $(S R R)$ such that,

$$
S R R=\frac{\# Y}{T}
$$

As for evaluating errors in $\hat{X}$ when compared to the original signal $X$, There are several metrics to chose from, however, in agreement with the arguments presented in [150] the metric I chose is the average Euclidean error (AEE) (sometimes called the mean absolute error, though it is not a recommended term according to [150]) denoted by $\overline{|e|}$ and calculated as,

$$
\overline{|e|}=\frac{1}{T} \sum_{t=1}^{T}\left|s_{t}-\hat{s}_{t}\right|
$$

In Figure 5.9, the performance of the four PSFs is compared. for the given signal S11, we can see the clear difference between the "low entropy" PSF and the other three. While the "low entropy" result exhibit the lowest $\overline{|e|}$ it is also saving no energy with the highest $S R R$. As for the other three PSFs, their performance is comparable with the "high entropy" function showing the lowest $S R R$ with a marginal increase in $\overline{|e|}$ over the "mirrored sigmoids" and the "high \& random". The highest value for $\overline{|e|}$ in this case is just below 0.015 . This means that with entropic sensing, we were able to recover $X$ in real-time with $98.5 \%$ accuracy while omitting more than $60 \%$ of samples from the data stream.

The plot in Figure 5.9 is a result corresponding only to $S 11$. The results for each of the chosen benchmarking series exhibit similar outcomes and are listed in Appendix 
B.4.

S11

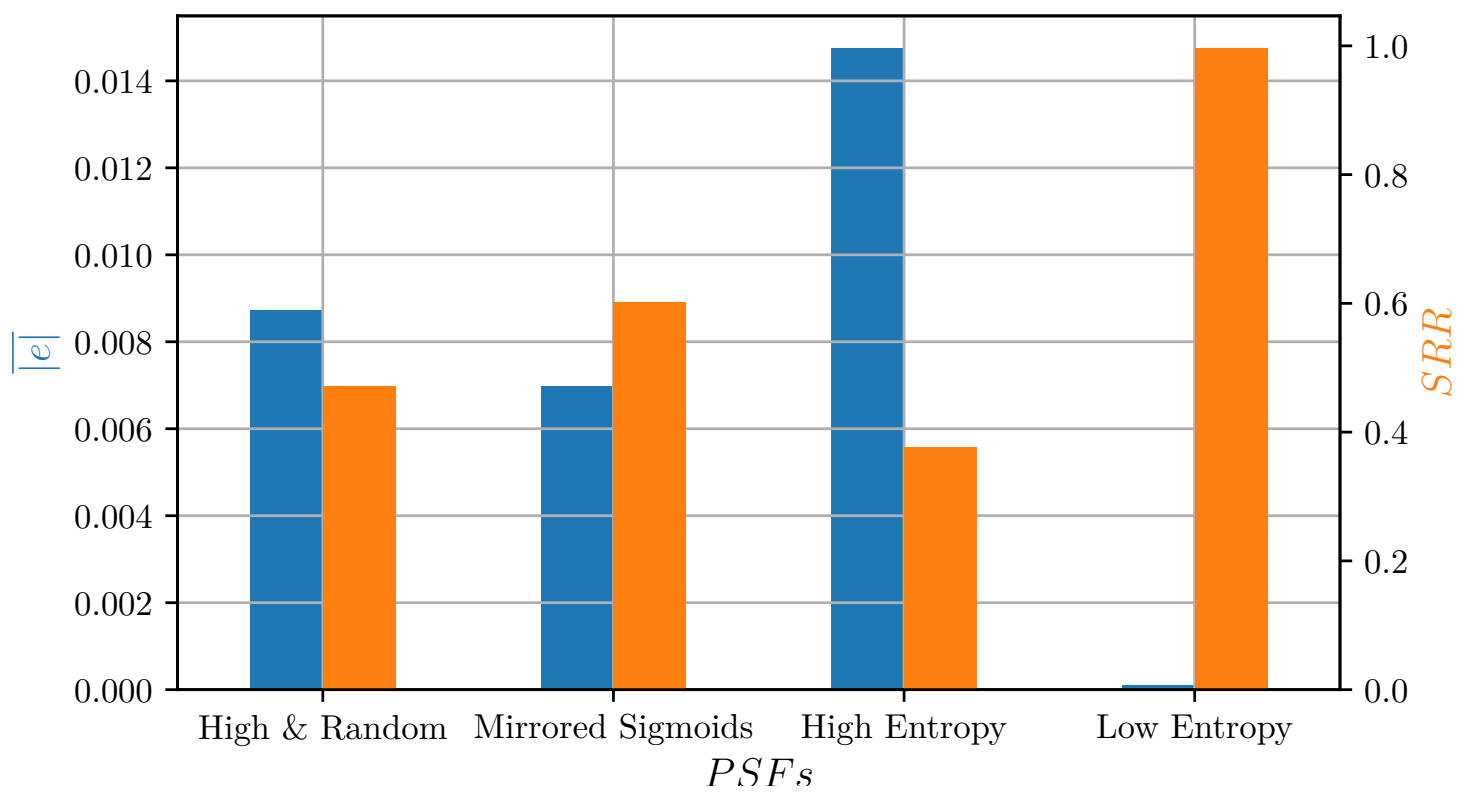

Figure 5.9: $\overline{|e|}$ and $S R R$ among all the probability shaping functions for $S 5$.

We also looked at the instantaneous absolute error $e_{t}=\left|s_{t}-\hat{s}_{t}\right|$ to get a sense of how errors occur over time. In Figure 5.10 we can see $X$ and $\hat{X}$ in the top plot and $\overline{|e|}$ in the bottom plot. Notice how $\overline{|e|}$ decreases over time as the prediction model gets better with incremental learning. The decreasing trend can be observed in some benchmarking signals such as $S 11$ while not in others such as $S 8$. The decreasing trend can be attributed to the non-increasing complexity of signals over time. The LSTM model is able to learn such complexity over time, and hence produce more accurate predictions.

We can see that signals with a random component such as $S 5$ to $S 8$ do not exhibit the decreasing trend in $\overline{|e|}$, because the predictor is not able to catch up with the 


\section{S11 with PSF=High Entropy}

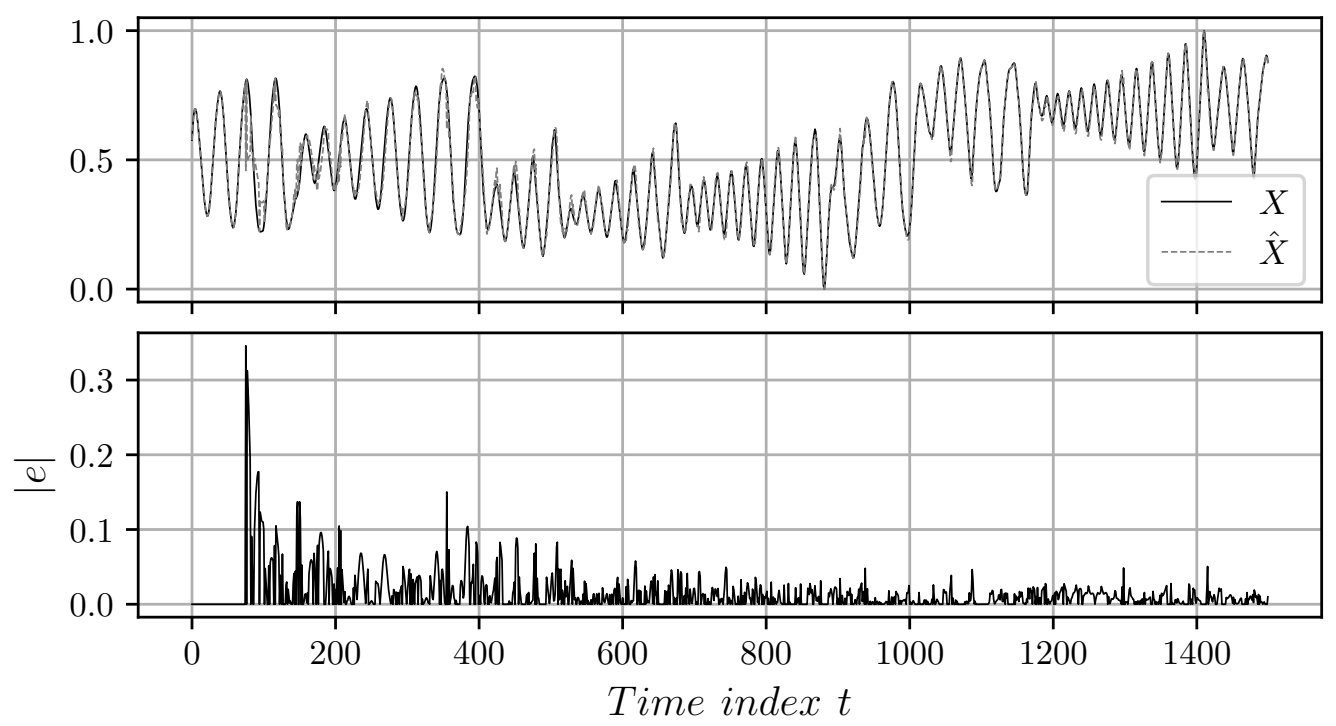

Figure 5.10: Top: $X$ and $\hat{X}$. Bottom: Instantaneous absolute error $|e|$.

increasing complexity of the signal over time. This is noticeable in the instantaneous entropy plots of such signals where we can see more fluctuations and no saturation as time passes. Furthermore, an increasing trend in $\overline{|e|}$ is demonstrated for $S 2$ as shown in Figure B.46 for the same reason. It is because $S 2$ does not cover its full dynamic range until the very end. This means new information is being added constantly to the predictor at a rate higher than its ability to learn. This can be deduced from the instantaneous entropy plot of $S 2$ in Figure B.13.

Like any parameterized algorithm there are some trade-offs to be made when we tune the parameters. If we vary the threshold th of $p s f 1$ we can bias the glsEF algorithm towards or away from high entropy samples as desired. This has a direct effect on $S R R$ and $\overline{|e|}$. As shown in Figure 5.11, as th increases, $S R R$ decreases because fewer and fewer samples lie in the high entropy side of the histograms of $\check{r}_{X}$ 


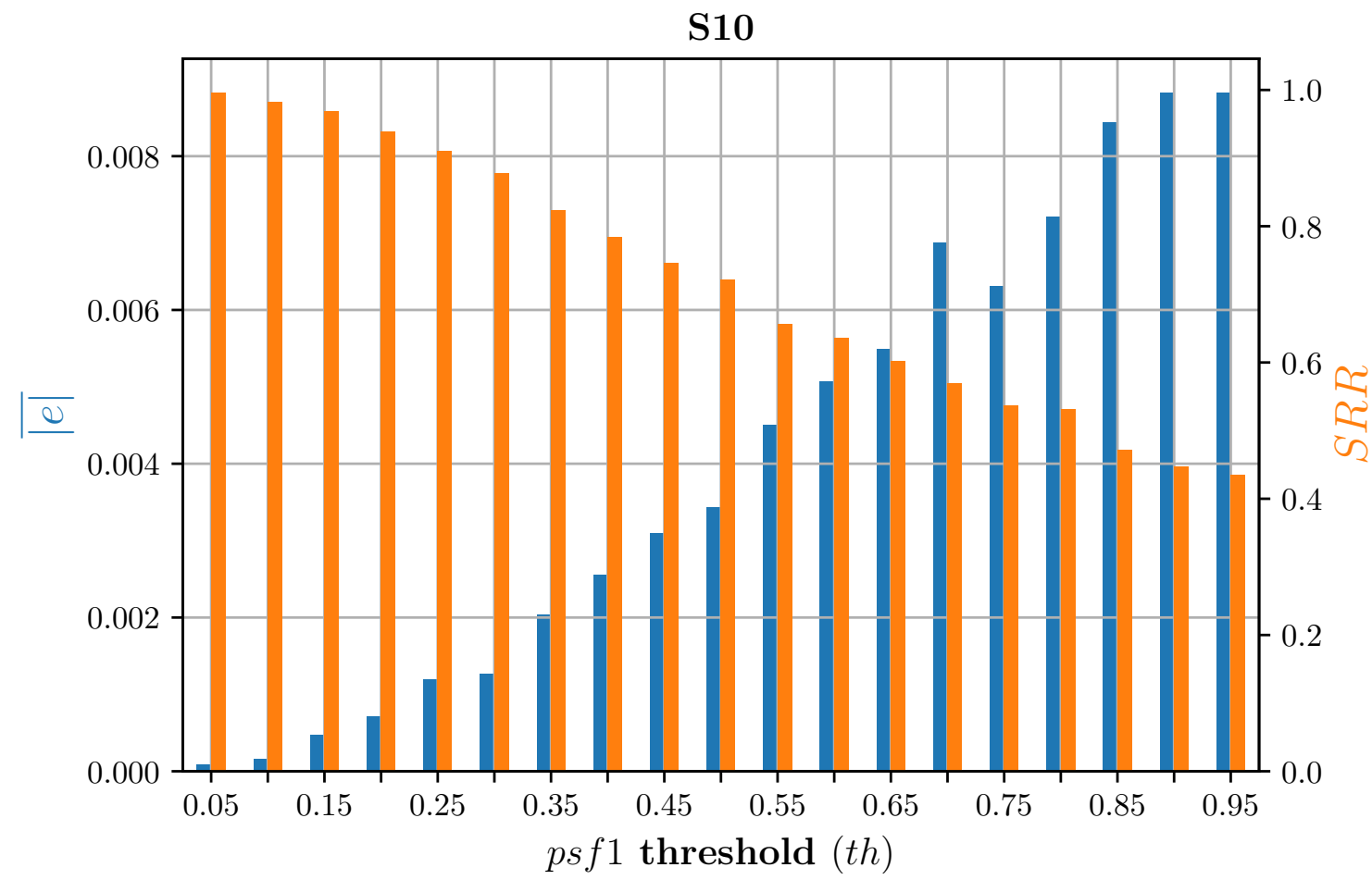

Figure 5.11: Average Euclidean error $(\overline{|e|})$ and the sample reduction ration $(S R R)$ vs the threshold $(t h)$ of $p s f 1$.

and $\check{r}_{d X}$ as shown in Figure 5.7. On the contrary, $\overline{|e|}$ increases with th because fewer samples means less information available for the predictor. However, even with a very high $t h=0.95$ the mean absolute error $\overline{|e|}$ is still limited below $0.9 \%$ supporting the claim that most of the self information in a time-series is contained in a small portion of its samples.

\subsubsection{Memory Requirements}

At every time step, the SN compares $\check{r}_{X}$ with $|d H(X)|_{1 \rightarrow t-1}$ and $\check{r}_{d X}$ with $|d H(d X)|_{1 \rightarrow t-1}$. This means the SN will have to store $2(T-1)$ entries by the end of its life. Also, we need to store the previous and current PMFs of $X$ which accounts for $2 N$ entries. 
Further memory needs are marginal and independent of sensory data.

In our case $T=1500$ and $N=50$. Thus, most of our memory needs can be accounted for by $2(1500-1)+2 \times 50=3098$ locations. Assuming we store all our entries in a standard floating-point format $(32 \mathrm{~b}=4 \mathrm{~B})$, the required memory size amounts to $\approx 3 \mathrm{~KB}$. This is highly reasonable for most currently available microcontrollers. For example, the microcontroller used in Chapter 4 (TI: MSP432P401R) has up to $64 \mathrm{~KB}$ of SRAM and $16 \mathrm{~KB}$ of flash information memory (excluding $256 \mathrm{~KB}$ of flash program memory).

\subsubsection{Energy Savings}

So far, the energy savings of the $\mathrm{SN}$ has been expressed in terms of the sample reduction ratio $S R R$. This section will translate the value of the $S R R$ to actual energy savings based on real measurements from our work in Chapter 4.

From Section 4.4, the energy consumption of our SN through one reporting cycle was measured to be $\approx 62 \mathrm{~mJ}$. From Chapter 4 , the 9-cell battery at a factor of safety 0.5 , gives a total capacity of $2.6 \times 9 \times 0.5=11.7 \mathrm{Ah}(151,632 \mathrm{~J})$. Therefore, the SN will

be able to report up to $\frac{151632}{0.062}=2,445,677$ samples throughout its lifetime. However with entropic sensing, an $S R R=0.4$, which is typical in the presented results, can extend the number of samples to be reported by $(1-0.4) \times 2445677=1,467,406$ bringing the total number to $3,913,083$. This accounts for a $60 \%$ increase in either lifetime or reporting time resolution whichever way we decide to utilize the energy surplus.

The factor governing the amount of energy saving for a given time series is its $S R R$. The $S R R$ depends on the structure of the given time series as well as the 
parameters of the entropic filter (e.g. the PSF).

\subsection{Summary}

Entropic sensing is an energy saving approach for wireless sensors in real-time monitoring applications. We defined instantaneous entropy and derived an efficient iterative formula to compute Shannon's entropy for a time-series.

We introduced the EF which allows the wireless sensor to transmit information rich samples only while omitting the others. The EF carries marginal computing overhead, and by extension marginal energy cost on the SN. The system's demonstrated potential shows more than $60 \%$ increase in the energy budget with less than $2 \%$ average Euclidean error in the recovered signal. The system can be configured in many ways to fit various scenarios and constraints.

Entropic sensing can be incorporated in existing SN hardware with a firmware update along with a software update to the sink. It does not require any hardware modifications, special communication protocols or network reconfiguration. Consequently, entropic sensing is compliant with existing communication standards by default.

SNs with multiple sensors onboard may not have similar energy savings because the intersection between the sets of omitted samples from different sensors may not result in a significant reduction in the number of transmitted data packets. In other words, when a sample from a sensor $x_{1 i}$ is omitted, it doesn't necessarily mean that the sample from another sensor $x_{2 i}$ will also be omitted. This means that the SN's radio will have to turn on and transmit a data packet every time step unless all samples from all sensors onboard are simultaneously omitted by their respective entropic filters. 
On the other hand, it is sensible to expect different time-series to exhibit similar entropic trends if all signals are related to the same physical entity (i.e., if they are somewhat correlated). In a more practical sense, if an $\mathrm{SN}$ is, for example, monitoring the internal temperature and pressure of a fuel tank we can expect to see no transmitworthy samples in the temperature series if the pressure series also has no transmitworthy samples at the same time. Furthermore, if there are any unusual behaviour in the temperature series, the pressure series will probably exhibit a similar behaviour. 


\section{Chapter 6}

\section{Conclusion}

This thesis has presented an integrated solution for monitoring Ground Engaging Tools (GET) and industrial assets in general. The designs put forward serve future developments by highlighting major challenges and exploring potential mitigation strategies.

The Sensor Node (SN) presented here consisted of nine sensors employing different technologies. This work combined off-the-shelf with custom-designed proximity sensors to enhance the redundancy of sensing the most important physical quantity in our application which is a proxy for the mechanical integrity of GET. Other sensors were included to monitor the SN during operation and provide context-awareness.

Wireless communication has been reliably established in a challenging situation where the $\mathrm{SN}$ is enclosed inside a non-hermetic metallic cavity. The experiments demonstrated the effect of antenna tuning on the Received Signal Strength (RSS). The field test demonstrated the signal's ability to penetrate the pile of sand effectively. While it did not confirm that it could propagate through several loads of oil sands, there is no technical reason to expect otherwise (except when the hardware integrity is compromised) because oil sands has low relative permittivity [151]. 
The packaging was able to sustain impact forces up to 8 ton-force without loss in functionality. Field tests have validated the system in practice and under real conditions. Such successful and documented endeavours can trigger future research and development in unprecedented directions and encourage researchers to cover new Industrial Internet of Things (IIoT) applications with a better idea about the associated risks and challenges.

Entropic sensing is introduced as an energy-efficient approach for wireless sensors in real-time monitoring applications. Instantaneous Entropy (IE) was defined and utilized as a simple way to quantify the rate of entropy accumulation in time-series. Moreover, we derived an iterative formula to efficiently compute Shannon's entropy for a time-series in real-time. We introduced the Entropic Filter (EF), which allows the wireless sensor to down sample a time-series and transmit information rich samples while omitting easily predictable samples. The EF carries marginal computing overhead and, by extension, marginal energy cost on the SN. The system's demonstrated potential shows more than a $60 \%$ reduction in energy consumption with no more than $2 \%$ mean absolute error in the recovered signal. The system is flexible and can be configured in many ways to fit various types of time-series.

The simulation of entropic sensing employed various types of benchmarking timeseries, including stationary, non-stationary, chaotic, and even a random walk. Potentially, this can help reduce the energy consumption of all wireless sensing devices with minimal effect on the accuracy of recovered data. Furthermore, such development can potentially invoke better approaches in time-series prediction, data compression, and low-power communication protocols. 


\subsection{Future Directions}

Moving forward, it is worth mentioning that implementing a system level solution such as the one in this thesis is a significant undertaking. Future research efforts should consider the various resources needed. Technical challenges are not the only challenges facing such projects. Managing logistics, components and material sourcing, and manufacturing are major aspects to be considered. Also, worth noting the time it takes to build a working prototype and test it in the field. Pursuing a practical implementation carries invaluable engineering lessons, however, its academic output is diluted.

In this section, we include a technical discussion of the future directions in each module based on the lessons learned from this work.

\subsubsection{Communication}

Wireless communication with an SN embedded inside a non-hermetic metallic en-

closure is a challenge facing deployments of Wireless Sensor Networks (WSNs) in industrial facilities. More research in electromagnetism is needed to find a generic solution that can fit most enclosures and allow the signal to propagate outside with minimal losses.

It could be possible to design a radiating structure small enough to fit within the tight clearances of industrial metallic enclosures yet robust enough to withstand mechanical abuse and extreme temperatures. This design will allow for SNs to be embedded in more industrial assets and equipment without having to custom design a specific antenna for each application. 


\subsubsection{Ferrous-Selective Magnetic Force Proximity Sensor}

Using a load-cell instead of the Force Sensing Resistor (FSR) can improve the sensor's accuracy, increase its dynamic range, and reduce its hysteresis significantly. If the component cost is justified, a load-cell will introduce several improvements in the sensor's performance.

Furthermore, the use of 3D printed fixtures during experiments has been shown to introduce inaccuracy because 3D printed plastic will flex under elevated loads. In the future, when characterizing the sensor's performance with a universal testing machine, it would be better to use a more rigid non-ferrous material for the fixtures.

\subsubsection{Wear-Level Sensor}

The parallel-plate capacitor followed the theoretical models only for about $100 \mathrm{~mm}$.

For the remainder of our probe's length, the measured capacitance diverges significantly from all the models. This divergence calls for a deeper investigation into the existing parallel-plate capacitor models to expose any physical effects ignored by our current theoretical models or possibly attribute the discrepancy to errors in either the manufacturing tolerances of our Printed Circuit Board (PCB) or the experimental setup.

\subsubsection{Capacitive Proximity Sensor}

In our presented design, we used a passive ground shield underneath the capacitive sensing pad. In the future, an active shield could be better suited for proximity applications where distance resolution could be sacrificed for a larger sensing range. The active shield consumes a little more power but, by turning off the sensing chip when 
it is not acquiring measurements, the extra power consumption could be reduced.

In the future, we recommend using another chip (TI: FDC1004) for capacitive sensing. The FDC1004 features an integrated active shield driver that can help reduce development costs.

\subsubsection{Entropic Sensing}

The Long Short Term Memory (LSTM) predictor builds its model on the past transmitted and predicted samples; this means that errors in the past predictions are incorporated in the model and, by extension, all future predictions. We may be able to reduce errors in $\hat{X}$ if we can configure the Long Short Term Memory (LSTM) predictor to update its model without considering past predicted samples (only transmitted ones). This way, we make sure that erroneous information about $X$ will not be incorporated into our prediction model.

Another aspect of entropic sensing that could benefit from further research is the optimization of the percentage of priming samples pps and how it affects the mean absolute error $\overline{|e|}$ and the sample reduction ratio $S R R$ of given signals.

Extending entropic sensing to reach the realm of practical applications hangs on its limitation to SNs with only one sensory data stream, that is one time-series. One might argue that for SNs monitoring the same physical state with multiple transducers, all time-series may exhibit the same instantaneous entropy profile over time. This profile is based on the assumption that interesting events will be represented in all time-series simultaneously. If that is true, we can easily utilize entropic sensing (with

some modifications) for all transducers onboard the SN without increasing the $S R R$ too much. The effect of correlations between multiple signals on the performance of 
their combined entropic sensing algorithm needs to be investigated in future work.

Furthermore, future extensions of this work may explore other entropy formulas to evaluate each sample in real-time to prime it for entropic filtering. It is essential to study any potential formula in terms of its computational load and its ability to reduce $S R R$ while increasing $\overline{|e|}$.

After the completion of this work we list the recommendation for future projects of this nature. These recommendations are withdrawn from lessons learned over time.

\subsection{Development Recommendations}

1. 3D printing in-house takes substantial time and effort to operate and maintain industrial grade 3D printing equipment and resources. Therefore, we recommend outsourcing this activity in similar future projects.

2. Prototyping multi-layer PCBs has become less expensive over the past years; however, PCB assembly remains a relatively expensive service for small quantities. Thus, I recommend using an in-house PCB assembly setup with a semiautomatic pick-and-place machine and a reflow oven to assemble PCB prototypes quickly and cost-efficiently.

3. Passive components come in various packages. I recommend using 0402 (1005 Metric) components because they strike the right balance between ease of assembly, form factor, and Electromagnetic Interference (EMI) performance.

4. Electromagnetic Interference (EMI) is a serious concern when commercializing a wireless device. Even if the PCB layout complexity is low, it is recommended using a four-layer PCB stack to reduce EMI induced by high-frequency signals. 
The recommended stack is as follows:

Top layer: Signal+Power

layer 2: Ground plane

layer 3: Ground plane

Bottom layer: Signal+Power

Only if the circuit is complex and a power plane is needed, use layer 2 or 3 as the power plane and keep signals on the top and the bottom layers surrounded by ground planes and stitching ground vias.

5. Referring to the clock frequency as a differentiating criterion for high-frequency signals is misleading. What counts is the rise or fall time (whichever is shorter) of the clock edge. As a rule of thumb, the bandwidth of a given signal can be estimated from its rise time as follows:

$$
B W=\frac{0.35}{T_{r}}
$$

Where $B W$ is the bandwidth in $\mathrm{GHz}$ and $T_{r}$ is the rise time in ns.

6. Oscillators are affected by temperature variations. For extreme operating temperatures use Temperature Compensated Oscillators (TCOs) to enhance the clock's stability in various operating temperatures.

7. Power integrity is important in battery powered circuits, to enhance the power delivery network, use decoupling capacitors with appropriate values to stabilize the voltage supply. 
8. Maintaining an internal record of previously vetted parts. This means maintaining a master Bill of materials (BOM) separately and allows BOMs of specific designs to inherit their attributes from the master BOM seamlessly with a simple script. This inventory will help keep a single source of truth for all vetted parts and will be of added value if made available to researchers.

9. Institute of Printed Circuits (IPC) standards are very helpful to PCB designers. They act as a good generic Design for Manufacturing (DFM) guide. For future projects, It is recommended to purchase the following three IPC standards:
(a) IPC-2221 B
(b) IPC-2222 B
(c) IPC-7351 B

10. Enough attention should be given to vetting and rephrasing statements in the application requirements document because, every time spent on this document can save significant amounts of time and effort during development.

11. Use well-established, widely used, and open-source software tools whenever possible. KiCAD is a good example for an electric Computer Aided Design (CAD) tool. 


\section{Bibliography}

[1] G. Hassan and H. S. Hassanein, "MoT: A Deterministic Latency MAC Protocol for Mission-Critical IoT Applications," in 14th International Wireless Communications Mobile Computing Conference (IWCMC), Jun. 2018, pp. 588-593.

[2] "P\&H Electric Rope Shovels - Surface Mining | Komatsu Mining Corp." https://mining.komatsu/surface-mining/electric-rope-shovels.

[3] R. van Kranenburg, "The Internet of Things: A Critique of Ambient Technology and the All-seeing Network of RFID," The Netherlands: Institute of Network Cultures, Amsterdam, Tech. Rep., 2007.

[4] "IoT market size worldwide 2017-2025," https://www.statista.com/statistics/976313/global-iot-market-size/.

[5] L. D. Xu, W. He, and S. Li, "Internet of Things in Industries: A Survey," IEEE Transactions on Industrial Informatics, vol. 10, no. 4, pp. 2233-2243, Nov. 2014.

[6] A. Čolaković and M. Hadžialić, "Internet of Things (IoT): A review of enabling technologies, challenges, and open research issues," Computer Networks, vol. 144, pp. 17-39, Oct. 2018. 
[7] V. C. Gungor and G. P. Hancke, "Industrial Wireless Sensor Networks: Challenges, Design Principles, and Technical Approaches," IEEE Transactions on Industrial Electronics, vol. 56, no. 10, pp. 4258-4265, Oct. 2009.

[8] C.-Y. Chong and S. Kumar, "Sensor networks: Evolution, opportunities, and challenges," Proceedings of the IEEE, vol. 91, no. 8, pp. 1247-1256, Aug. 2003.

[9] N. Srivastava, "Challenges of Next-Generation Wireless Sensor Networks and its impact on Society," Journal of Telecommunications, vol. 1, no. 1, pp. pp128-133, 2010.

[10] A. Sharif, Ed., Harsh Environment Electronics: Interconnect Materials and Performance Assessment, 1st ed. Wiley-VCH, Aug. 2019.

[11] M. Healy, T. Newe, and E. Lewis, "Wireless Sensor Node hardware: A review," in IEEE SENSORS, Oct. 2008, pp. 621-624.

[12] H. Lasi, P. Fettke, H.-G. Kemper, T. Feld, and M. Hoffmann, "Industry 4.0," Business 83 Information Systems Engineering, vol. 6, no. 4, pp. 239-242, Aug. 2014 .

[13] Y. Lu, "Industry 4.0: A survey on technologies, applications and open research issues," Journal of Industrial Information Integration, vol. 6, pp. 1-10, Jun. 2017.

[14] K. Zhou, T. Liu, and L. Zhou, "Industry 4.0: Towards future industrial opportunities and challenges," in 12th International Conference on Fuzzy Systems and Knowledge Discovery (FSKD), Aug. 2015, pp. 2147-2152. 
[15] P. F. Cortese, F. Gemmiti, B. Palazzi, M. Pizzonia, and M. Rimondini, "Efficient and practical authentication of PUF-based RFID tags in supply chains," in 2010 IEEE International Conference on RFID-Technology and Applications, Jun. 2010, pp. 182-188.

[16] A. Buffi, B. Tellini, A. Motroni, and P. Nepa, "A Phase-based Method for UHF RFID Gate Access Control," in 2019 IEEE International Conference on RFID Technology and Applications (RFID-TA), Sep. 2019, pp. 131-135.

[17] U. Ketprom, C. Mitrpant, P. Makhapun, S. Makwimanloy, and S. Laokok, "RFID for Cattle Traceability System at Animal Checkpoint," in 2011 Annual SRII Global Conference, Mar. 2011, pp. 517-521.

[18] L. Vojtech, A. M. F. Lopez, M. Neruda, and Z. Lokaj, "Embedded system with RFID technology and inductive proximity sensor," in 36th International Conference on Telecommunications and Signal Processing (TSP), Jul. 2013, pp. 213-217.

[19] Z. Yuan and D. Huang, "A novel RFID-based shipping containers location and identification solution in multimodal transport," in 2008 Canadian Conference on Electrical and Computer Engineering, May 2008, pp. 000 267-000 272.

[20] J. Melià-Seguí, J. Garcia-Alfaro, and J. Herrera-Joancomartí, "RFID EPCGen2 for postal applications: A security and privacy survey," in 2010 IEEE International Conference on RFID-Technology and Applications, Jun. 2010, pp. $118-123$. 
[21] W. Yao, C.-H. Chu, and Z. Li, "The use of RFID in healthcare: Benefits and barriers," in 2010 IEEE International Conference on RFID-Technology and Applications, Jun. 2010, pp. 128-134.

[22] "ZIGBEE SPECIFICATION," Jul. 2012.

[23] "Connectivity Standards Alliance," https://zigbeealliance.org/about/.

[24] P. Cheong, K.-F. Chang, Y.-H. Lai, S.-K. Ho, I.-K. Sou, and K.-W. Tam, "A ZigBee-Based Wireless Sensor Network Node for Ultraviolet Detection of Flame," IEEE Transactions on Industrial Electronics, vol. 58, no. 11, pp. 5271-5277, Nov. 2011.

[25] T. de Almeida Oliveira and E. P. Godoy, "ZigBee Wireless Dynamic Sensor Networks: Feasibility Analysis and Implementation Guide," IEEE Sensors Journal, vol. 16, no. 11, pp. 4614-4621, Jun. 2016.

[26] M. Tao, X. Hong, C. Qu, J. Zhang, and W. Wei, "Fast access for ZigBeeenabled IoT devices using raspberry Pi," in 2018 Chinese Control And Decision Conference (CCDC), Jun. 2018, pp. 4281-4285.

[27] "Bluetooth," ITNOW, vol. 42, no. 3, pp. 32-32, May 2000.

[28] "Bluetooth Technology Website | The official website of Bluetooth technology." https://www.bluetooth.com/.

[29] "LoRa Alliance," https://lora-alliance.org/.

[30] "Valley Blades Limited," http://www.valleyblades.com/. 
[31] C. Subramanian, "Fatigue Failure of a Ground-Engaging Tool," Journal of Failure Analysis and Prevention, vol. 9, no. 2, pp. 122-126, Apr. 2009.

[32] G. Saha, "Abrasive wear of alloys for ground engaging tools," Ph.D. dissertation, Deakin University, Sep. 2017.

[33] P. Burke, "When the best laid plans go awry," MineSafe Magazine, vol. 16, no. 3, pp. 33-34, Dec. 2007.

[34] M. A. Ibrahim, G. Hassan, H. S. Hassanein, and K. Obaia, "A wireless sensor platform for industrial non-hermetic metallic enclosures," in 13th International Wireless Communications and Mobile Computing Conference (IWCMC), Jun. 2017, pp. 165-170.

[35] M. A. Ibrahim, G. Hassan, K. Monea, H. S. Hassanein, and K. Obaia, "A Ferrous-Selective Proximity Sensor for Industrial Internet of Things," in IEEE International Conference on Communications (ICC), Jun. 2020, pp. 1-6.

[36] M. A. Ibrahim, G. Hassan, H. S. Hassanein, and K. Obaia, "Wireless Sensing System for Monitoring Ground Engaging Tools," IEEE Internet of Things Journal, Submitted May 9, 2021.

[37] M. A. Ibrahim and H. S. Hassanein, "Entropic Sensing for Energy Efficiency," in IEEE International Conference on Mobile Ad Hoc and Smart Systems (MASS), Oct. 2021.

[38] M. A. Ibrahim, G. Hassan, H. S. Hassanein, and K. Obaia, "Wireless Sensing for Ground Engaging Tools," in GLOBECOM - IEEE Global Communications Conference, Dec. 2020, pp. 1-6. 
[39] H. Yamamoto, S. Minomi, K. Nakamura, and K. Yamazaki, "Development of Arkas Sensor Platform and Application for Heavy Snowfall Observation," in IEEE/IPSJ 12th International Symposium on Applications and the Internet (SAINT), Jul. 2012, pp. 399-404.

[40] A. Sieber, M. F. Wagner, J. Markert, and C. Wögerer, "Low power sensor platform for environmental monitoring," in IEEE Sensors, Oct. 2009, pp. $1804-1809$.

[41] H. Ramamurthy, B. S. Prabhu, R. Gadh, and A. M. Madni, "Wireless Industrial Monitoring and Control Using a Smart Sensor Platform," IEEE Sensors Journal, vol. 7, no. 5, pp. 611-618, May 2007.

[42] M. Tanevski, F. Merli, A. Boegli, A. K. Skrivervik, J. F. Zurcher, and P. A. Farine, "RoSe: A Subgigahertz Wireless Sensor Platform With HousingIntegrated Overmolded Antenna," IEEE Transactions on Instrumentation and Measurement, vol. 61, no. 11, pp. 2982-2992, Nov. 2012.

[43] M. Merenda, C. Felini, and F. G. D. Corte, "An autonomous and energy efficient Smart Sensor Platform," in IEEE SENSORS Proceedings, Nov. 2014, pp. $1208-1211$.

[44] C. C. Enz, A. El-Hoiydi, J. D. Decotignie, and V. Peiris, "WiseNET: An ultralow-power wireless sensor network solution," Computer, vol. 37, no. 8, pp. 62-70, Aug. 2004. 
[45] A. E. Kouche, "WIRELESS SENSOR NETWORK PLATFORM FOR HARSH INDUSTRIAL ENVIRONMENTS," Ph.D. dissertation, Queen's University, Kingston, ON, CANADA, Sep. 2013.

[46] _ _ " "Towards a wireless sensor network platform for the Internet of Things: Sprouts WSN platform," in IEEE International Conference on Communications (ICC), Jun. 2012, pp. 632-636.

[47] A. E. Kouche, H. S. Hassanein, and K. Obaia, "WSN platform Plug-and-Play (PnP) customization," in IEEE Ninth International Conference on Intelligent Sensors, Sensor Networks and Information Processing (ISSNIP). Singapore: IEEE, Apr. 2014, pp. 1-6.

[48] A. El Kouche, H. S. Hassanein, and A. Alma'aitah, "Configuring a UHF antenna for sensor nodes in harsh environments," in 27th Biennial Symposium on Communications (QBSC). Kingston, ON, Canada: IEEE, Jun. 2014, pp. $66-70$.

[49] A. Alma'aitah and H. S. Hassanein, "Utilizing Sprouts WSN platform for equipment detection and localization in harsh environments," in 39th Annual IEEE Conference on Local Computer Networks Workshops, Sep. 2014, pp. 777-783.

[50] A. E. Kouche, H. Hassanein, and K. Obaia, "Monitoring the reliability of industrial equipment using wireless sensor networks," in 8th International Wireless Communications and Mobile Computing Conference (IWCMC). Limassol, Cyprus: IEEE, Aug. 2012, pp. 88-93. 
[51] A. El Kouche, A. Alma'aitah, H. Hassanein, and K. Obaia, "Monitoring operational mining equipment using Sprouts Wireless Sensor Network platform," in 9th International Wireless Communications and Mobile Computing Conference (IWCMC). Sardinia, Italy: IEEE, Jul. 2013, pp. 1388-1393.

[52] E. P. Capo-Chichi and J. M. Friedt, "Design of embedded sensor platform for multimedia application," in First International Conference on Distributed Framework and Applications, Oct. 2008, pp. 146-150.

[53] R. Khoie, B. Gopaluni, J. A. Olson, and B. Stoeber, "A magnetic sensor to measure wear in centrifugal pumps," in IEEE SENSORS, Nov. 2015, pp. 1-4.

[54] S. M. zu Hoberge, U. Hilleringmann, C. Jochheim, and M. Liebich, "Piezoelectric sensor array with evaluation electronic for counting grains in seed drills," in IEEE Africon '11, Sep. 2011, pp. 1-6.

[55] M. I. M. Ismail, R. A. Dziyauddin, and N. A. A. Samad, "Water pipeline monitoring system using vibration sensor," in IEEE Conference on Wireless Sensors (ICWiSE), Oct. 2014, pp. 79-84.

[56] G. Wei, J. W. Gardner, M. Cole, and Y. Xing, "Multi-sensor module for a mobile robot operating in harsh environments," in IEEE SENSORS, Oct. 2016, pp. 1-3.

[57] S. Mekid, U. Baroudi, and A. Bouhraoua, "Stand Alone Smart Bolt for eMaintenance," International Journal of Engineering and Technology, vol. 4, no. 5, pp. 577-580, 2012.

[58] "RISC-V," https://riscv.org/. 
[59] M. A. Ibrahim, G. Hassan, H. S. Hassanein, and K. Obaia, "Wireless Sensing for Ground Engaging Tools," in IEEE Global Communications Conference, Taipei, Taiwan, Dec. 2020.

[60] J. W. Betournay, N. D. Cowgill, S. D. HYDE, C. M. Carpenter, and J. M. ANDERTON, "Monitoring ground engaging products," WO Patent WO2 020257 209A1, Dec., 2020.

[61] N. Hillier, S. M. S. Shrestha, R. BAtTen, and M. JUnG, "Ground engaging tool monitoring system," WO Patent WO2 020237 324A1, Dec., 2020.

[62] C. M. Carpenter, K. D. ZUEndeL, N. D. Cowgill, J. K. Hoyt, and D. J. SAYLER, "Monitoring ground-engaging products for earth working equipment," WO Patent WO2 016131 007A1, Aug., 2016.

[63] J. M. Plouzek, M. C. VLAMINCK, and N. S. Finch, "Ground engaging tool monitoring system," WO Patent WO2 020231 555A1, Nov., 2020.

[64] "Motion Metrics," https://www.motionmetrics.com/.

[65] M. Ramezani and S. Tafazoli, "Using Artificial Intelligence in Mining Excavators: Automating Routine Operational Decisions," IEEE Industrial Electronics Magazine, vol. 15, no. 1, pp. 6-11, Mar. 2021.

[66] T. M. Finley, C. M. Carpenter, and E. E. Wilkinson, "Ground engaging tool management," US Patent US20 170352 199A1, Dec., 2017.

[67] A. Al-Fagih, A. E. Kouche, S. Oteafy, and A. Alma'aitah, "Utilizing RFIDWSNs for reducing the footprint of the Oil Sands industry," in IEEE Globecom Workshops. Anaheim, CA, USA: IEEE, Dec. 2012, pp. 374-379. 
[68] E. L. Bewley, N. Cowgill, and J. E. Blomberg, "Wear part monitoring," US Patent US9 670 649B2, Jun., 2017.

[69] "G.E.T. Detect," http://escocorp.com/EN/smartproducts/Pages/GETDetect.aspx.

[70] T. L. ATKInSON, M. J. Behmlander, J. R. Hammar, and R. L. Meyer, "Erosion monitoring system for ground engaging tool," AU Patent AU2 014 254370B2, Jul., 2018.

[71] B. DARLington, B. KNOWLES, P. SCHILD, and B. DALLARD, "Attachment status monitoring of ground engaging tools (get) at heavy machinery," WO Patent WO2 018095 536A1, May, 2018.

[72] J. M. Kenchington and C. R. Phillips, "Operating Cost Parameters in Solvent Extraction of Bitumen from Oil Sand Mineral Deposits," Energy Sources, vol. 5, no. 4, pp. 317-338, Jan. 1981.

[73] thingsboard, "ThingsBoard - Open-source IoT Platform," https://thingsboard.io/.

[74] "Twilio - Communication APIs for SMS, Voice, Video and Authentication," https://www.twilio.com/.

[75] G. Hassan, M. ElMaradny, M. A. Ibrahim, A. M. Rashwan, and H. S. Hassanein, "Energy Efficiency Analysis of Centralized-Synchronous LoRa-based MAC Protocols," in 14th International Wireless Communications Mobile Computing Conference (IWCMC), Jun. 2018, pp. 999-1004. 
[76] D.-X. Yang, Z. Hu, H. Zhao, H.-F. Hu, Y.-Z. Sun, and B.-J. Hou, "ThroughMetal-Wall Power Delivery and Data Transmission for Enclosed Sensors: A Review," Sensors (Basel, Switzerland), vol. 15, no. 12, pp. 31 581-31605, Dec. 2015 .

[77] Andrea Goldsmith, Wireless Communications. Cambridge University Press, 2005.

[78] Z. Popovic and B. D. Popovic, Introductory Electromagnetics. USA: Prentice Hall, 2000.

[79] S. J. Chapman, D. P. Hewett, and L. N. Trefethen, "Mathematics of the Faraday Cage," SIAM Review, vol. 57, no. 3, pp. 398-417, Jan. 2015.

[80] “AN1200.22: LoRa ${ }^{\mathrm{TM}}$ Modulation Basics," May 2015.

[81] C. Canali, G. D. Cicco, B. Morten, M. Prudenziati, and A. Taroni, "A Temperature Compensated Ultrasonic Sensor Operating in Air for Distance and Proximity Measurements," IEEE Transactions on Industrial Electronics, vol. IE-29, no. 4, pp. 336-341, Nov. 1982.

[82] P. Wei and L. Zhizeng, "A Design of Miniature Strong Anti-jamming Proximity Sensor," in International Conference on Computer Science and Electronics Engineering, vol. 3, Mar. 2012, pp. 327-331.

[83] "Project Soli," https://atap.google.com/soli/.

[84] J. Choi and O. Choi, "Integrated visual sensor with 2D/3D imaging and in-situ proximity sensing for mobile devices," Electronics Letters, vol. 52, no. 16, pp. 1377-1379, 2016. 
[85] R. Bhattacharyya, C. Floerkemeier, and S. Sarma, "Low-Cost, Ubiquitous RFID-Tag-Antenna-Based Sensing," Proceedings of the IEEE, vol. 98, no. 9, pp. 1593-1600, Sep. 2010.

[86] Zhenhai Chen and R. C. Luo, "Design and implementation of capacitive proximity sensor using microelectromechanical systems technology," IEEE Transactions on Industrial Electronics, vol. 45, no. 6, pp. 886-894, Dec. 1998.

[87] S. Muhlbacher-Karrer, A. Gaschler, and H. Zangl, "Responsive fingers - capacitive sensing during object manipulation," in IEEE/RSJ International Conference on Intelligent Robots and Systems (IROS). Hamburg, Germany: IEEE, Sep. 2015, pp. 4394-4401.

[88] P. Ripka, J. Vyhnanek, M. Janosek, and J. Vcelak, "AMR Proximity Sensor With Inherent Demodulation," IEEE Sensors Journal, vol. 14, no. 9, pp. 3119-3123, Sep. 2014.

[89] S. Fericean and R. Droxler, "New Noncontacting Inductive Analog Proximity and Inductive Linear Displacement Sensors for Industrial Automation," IEEE Sensors Journal, vol. 7, no. 11, pp. 1538-1545, Nov. 2007.

[90] A. A. Eddib and D. D. L. Chung, "Electric permittivity of carbon fiber," Carbon, vol. 143, pp. 475-480, Mar. 2019.

[91] A. Matute, L. Paredes-Madrid, E. Gutierrez, and C. A. P. Vargas, "Characterization of drift and hysteresis errors in force sensing resistors considering their piezocapacitive effect," in IEEE SENSORS, Oct. 2017, pp. 1-3. 
[92] S. I. Yaniger, "Force Sensing Resistors: A Review Of The Technology," in Electro International, 1991, Apr. 1991, pp. 666-668.

[93] O. C. Fawole and M. Tabib-Azar, "Electromechanically-modulated permanent magnet antennas for wireless communication," in IEEE SENSORS, Oct. 2017, pp. $1-3$.

[94] F. Xia, B. Bahreyni, and F. Campi, "Multi-functional capacitive proximity sensing system for industrial safety applications," in IEEE SENSORS, Oct. 2016, pp. 1-3.

[95] V. K. Sadagopan, U. Rajendran, and A. J. Francis, "Anti theft control system design using embedded system," in Proceedings of IEEE International Conference on Vehicular Electronics and Safety, Jul. 2011, pp. 1-5.

[96] A. Shahidi, L. A. Gupta, A. Kovacs, and D. Peroulis, "Wireless temperature and vibration sensor for real-time bearing condition monitoring," in IEEE MTT-S International Microwave Symposium Digest (MTT), Jun. 2013, pp. 1-4.

[97] Y. Wei, R. Torah, Y. Li, and J. Tudor, "Dispenser printed proximity sensor on fabric for creative smart fabric applications," in Symposium on Design, Test, Integration and Packaging of MEMS/MOEMS (DTIP), Apr. 2015, pp. 1-4.

[98] “Texas instruments: FDC2214,” Jun. 2015.

[99] T. R. Jensen, R. G. Radwin, and J. G. Webster, "A conductive polymer sensor for measuring external finger forces," Journal of Biomechanics, vol. 24, no. 9, pp. 851-858, Jan. 1991. 
[100] L. Paredes-Madrid, A. Matute, and A. Peña, "Framework for a CalibrationLess Operation of Force Sensing Resistors at Different Temperatures," IEEE Sensors Journal, vol. 17, no. 13, pp. 4133-4142, Jul. 2017.

[101] L. K. Baxter, Capacitive Sensors: Design and Applications, ser. IEEE Press Series on Electronics Technology. IEEE, 1997.

[102] X. Hu and W. Yang, "Planar capacitive sensors - designs and applications," Sensor Review, vol. 30, no. 1, pp. 24-39, Jan. 2010.

[103] thingsboard, "ThingsBoard - Open-source IoT Platform," https://thingsboard.io/.

[104] A. A. a. F. Government of Alberta, "Weather Data for Alberta Townships by Alberta Climate Information Service (ACIS)." https://agriculture.alberta.ca/acis/township-data-viewer.jsp.

[105] Y. Song and H. Yu, "A RSS Based Indoor Tracking Algorithm via Particle Filter and Probability Distribution," in 4th International Conference on Wireless Communications, Networking and Mobile Computing, Oct. 2008, pp. 1-4.

[106] X. Chen, Z. Zhang, S. Yu, and T.-G. Zsurzsan, "Fringing Effect Analysis of Parallel Plate Capacitors for Capacitive Power Transfer Application," in IEEE 4th International Future Energy Electronics Conference (IFEEC). Singapore, Singapore: IEEE, Nov. 2019, pp. 1-5.

[107] M. Bao, Analysis and Design Principles of MEMS Devices, M. Bao, Ed. Amsterdam: Elsevier Science, Jan. 2005. 
[108] V. Hutson, "The circular plate condenser at small separations," Mathematical Proceedings of the Cambridge Philosophical Society, vol. 59, no. 1, pp. 211-224, Jan. 1963.

[109] A. El Kouche, A. M. Rashwan, and H. Hassanein, "Energy consumption measurements and reduction of Zigbee based wireless sensor networks," in IEEE Global Communications Conference (GLOBECOM), Dec. 2013, pp. 557-562.

[110] J. Song and Y. K. Tan, "Energy consumption analysis of ZigBee-based energy harvesting wireless sensor networks," in IEEE International Conference on Communication Systems (ICCS), Nov. 2012, pp. 468-472.

[111] J. Lu, H. Okada, T. Itoh, T. Harada, and R. Maeda, "Toward the World Smallest Wireless Sensor Nodes With Ultralow Power Consumption," IEEE Sensors Journal, vol. 14, no. 6, pp. 2035-2041, Jun. 2014.

[112] F. Marcelloni and M. Vecchio, "A Simple Algorithm for Data Compression in Wireless Sensor Networks," IEEE Communications Letters, vol. 12, no. 6, pp. 411-413, Jun. 2008.

[113] C. M. Sadler and M. Martonosi, "Data compression algorithms for energyconstrained devices in delay tolerant networks," in Proceedings of the 4th International Conference on Embedded Networked Sensor Systems - SenSys '06. Boulder, Colorado, USA: ACM Press, 2006, p. 265.

[114] A. Y. Carmi, L. Mihaylova, and S. J. Godsill, Eds., Compressed Sensing \& Sparse Filtering, ser. Signals and Communication Technology. Berlin, Heidelberg: Springer Berlin Heidelberg, 2014. 
[115] D. L. Donoho, "Compressed sensing," IEEE Transactions on Information Theory, vol. 52, no. 4, pp. 1289-1306, Apr. 2006.

[116] D. Donoho, M. Vetterli, R. DeVore, and I. Daubechies, "Data compression and harmonic analysis," IEEE Transactions on Information Theory, vol. 44, no. 6, pp. 2435-2476, Oct. 1998.

[117] L. Wang, K. Lu, and P. Liu, "Compressed Sensing of a Remote Sensing Image Based on the Priors of the Reference Image," IEEE Geoscience and Remote Sensing Letters, vol. 12, no. 4, pp. 736-740, Apr. 2015.

[118] R. van Sloun, A. Pandharipande, M. Mischi, and L. Demi, "Compressed Sensing for Ultrasound Computed Tomography," IEEE Transactions on Biomedical Engineering, vol. 62, no. 6, pp. 1660-1664, Jun. 2015.

[119] J. Zhao, Q. Liu, X. Wang, and S. Mao, "Scheduled Sequential Compressed Spectrum Sensing for Wideband Cognitive Radios," IEEE Transactions on Mobile Computing, vol. 17, no. 4, pp. 913-926, Apr. 2018.

[120] F. Biondi, "Compressed sensing radar - new concepts of incoherent continuous wave transmissions," in 3rd International Workshop on Compressed Sensing Theory and Its Applications to Radar, Sonar and Remote Sensing (CoSeRa), Jun. 2015, pp. 204-208.

[121] M. S. Asif and J. Romberg, "Dynamic updating for sparse time varying signals," in 43rd Annual Conference on Information Sciences and Systems, Mar. 2009, pp. 3-8. 
[122] D. Angelosante, G. B. Giannakis, and E. Grossi, "Compressed sensing of timevarying signals," in 16th International Conference on Digital Signal Processing, Jul. 2009, pp. 1-8.

[123] A. Korlekar, U. Pacharaney, and R. K. Gupta, "Energy efficient information gathering in wireless sensor networks using compressive sensing," in International Conference on Information Communication and Embedded Systems (ICICES), Feb. 2016, pp. 1-8.

[124] T. M. Cover and J. A. Thomas, Elements of Information Theory, 2nd ed. Wiley Interscience, 2006.

[125] C. E. Shannon, "A mathematical theory of communication," The Bell System Technical Journal, vol. 27, no. 3, pp. 379-423, Jul. 1948.

[126] A. Rényi, "On Measures of Entropy and Information," in Proceedings of the Fourth Berkeley Symposium on Mathematical Statistics and Probability, Volume 1: Contributions to the Theory of Statistics. The Regents of the University of California, 1961.

[127] D. Faddejew, "Zum begriff der entropie eines endlichen wahrscheinlichkeitsschemas," Arbeiten zur Informationstheorie I, p. 86, 1957.

[128] G. Pastor, I. Mora-Jimenez, R. Jantti, and A. Caamano, "Constructing Measures of Sparsity," IEEE Transactions on Knowledge and Data Engineering, pp. $1-1,2020$. 
[129] G. Pastor, I. Mora-Jimenez, R. Jantti, and A. J. Caamano, "Sparsity-Based Criteria for Entropy Measures," in The Tenth International Symposium on Wireless Communication Systems (ISWCS)), Aug. 2013, pp. 1-5.

[130] A. Rényi, "On Measures of Entropy and Information," in Proceedings of the Fourth Berkeley Symposium on Mathematical Statistics and Probability, Volume 1: Contributions to the Theory of Statistics. The Regents of the University of California, 1961.

[131] S. M. Pincus, "Approximate entropy as a measure of system complexity." Proceedings of the National Academy of Sciences, vol. 88, no. 6, pp. 2297-2301, Mar. 1991.

[132] S. M. Pincus and A. L. Goldberger, "Physiological time-series analysis: What does regularity quantify?" American Journal of Physiology-Heart and Circulatory Physiology, vol. 266, no. 4, pp. H1643-H1656, Apr. 1994.

[133] S. Pincus and R. E. Kalman, "Irregularity, volatility, risk, and financial market time series," Proceedings of the National Academy of Sciences of the United States of America, vol. 101, no. 38, pp. 13 709-13 714, Sep. 2004.

[134] A. Delgado-Bonal, A. Marshak, Y. Yang, and D. Holdaway, "Analyzing changes in the complexity of climate in the last four decades using MERRA-2 radiation data," Scientific Reports, vol. 10, no. 1, p. 922, Jan. 2020.

[135] A. Delgado-Bonal and A. Marshak, "Approximate Entropy and Sample Entropy: A Comprehensive Tutorial," Entropy, vol. 21, no. 6, p. 541, Jun. 2019. 
[136] M. Costa, A. L. Goldberger, and C.-K. Peng, "Multiscale Entropy Analysis of Complex Physiologic Time Series," Physical Review Letters, vol. 89, no. 6, p. 068102, Jul. 2002.

[137] _ _ "Multiscale entropy analysis of biological signals," Physical Review E, vol. 71, no. 2, p. 021906, Feb. 2005.

[138] C. Bandt and B. Pompe, "Permutation Entropy: A Natural Complexity Measure for Time Series," Physical Review Letters, vol. 88, no. 17, Apr. 2002.

[139] L. Zunino, F. Olivares, F. Scholkmann, and O. A. Rosso, "Permutation entropy based time series analysis: Equalities in the input signal can lead to false conclusions," Physics Letters A, vol. 381, no. 22, pp. 1883-1892, Jun. 2017.

[140] W. Bajwa, J. Haupt, A. Sayeed, and R. Nowak, "Compressive Wireless Sensing," in Proceedings of the 5th International Conference on Information Processing in Sensor Networks, ser. IPSN '06. New York, NY, USA: ACM, 2006, pp. $134-142$.

[141] "McNames Data Sets," https://web.cecs.pdx.edu/ mcnames/DataSets/index.html.

[142] S. R. Abbas and M. Arif, "New Time Series Predictability Metrics for Nearest Neighbor Based Forecasting," in IEEE International Multitopic Conference, Dec. 2006, pp. 100-105.

[143] M. Hénon, "A two-dimensional mapping with a strange attractor," Communications in Mathematical Physics, vol. 50, no. 1, pp. 69-77, Feb. 1976. 
[144] M. C. Shewry and H. P. Wynn, "Maximum entropy sampling," Journal of Applied Statistics, vol. 14, no. 2, pp. 165-170, Jan. 1987.

[145] K. Miranda, V. M. Ramos R., and T. Razafindralambo, "Using efficiently autoregressive estimation in wireless sensor networks," in International Conference on Computer, Information and Telecommunication Systems (CITS), May 2013, pp. $1-5$.

[146] D. Liu, "Open-loop training of recurrent neural networks for nonlinear dynamical system identification," in IJCNN'01. International Joint Conference on Neural Networks. Proceedings (Cat. No.01CH37222), vol. 2, Jul. 2001, pp. $1215-1220$ vol.2.

[147] S. D. Kumar and D. Subha, "Prediction of Depression from EEG Signal Using Long Short Term Memory(LSTM)," in 3rd International Conference on Trends in Electronics and Informatics (ICOEI), Apr. 2019, pp. 1248-1253.

[148] M. A. I. Sunny, M. M. S. Maswood, and A. G. Alharbi, "Deep Learning-Based Stock Price Prediction Using LSTM and Bi-Directional LSTM Model," in 2nd Novel Intelligent and Leading Emerging Sciences Conference (NILES), Oct. 2020, pp. 87-92.

[149] J. Jo, S. Hwang, S. Lee, and Y. Lee, "Multi-Mode LSTM Network for Energy-Efficient Speech Recognition," in International SoC Design Conference (ISOCC), Nov. 2018, pp. 133-134. 
[150] X. R. Li and Z. Zhao, "Measures of performance for evaluation of estimators and filters," in International Symposium on Optical Science and Technology, O. E. Drummond, Ed., San Diego, CA, USA, Nov. 2001, p. 530.

[151] F. S. Chute, F. E. Vermeulen, M. R. Cervenan, and F. J. McVea, "Electrical properties of Athabasca oil sands," Canadian Journal of Earth Sciences, vol. 16, no. 10, pp. 2009-2021, 1979. 


\section{Appendix A}

\section{Circuit Diagrams}

Each one of the two PCBs designed in this thesis has its schematic diagram listen in this Appendix. Each schematic is divided into three sheets. Sheet 1 is the master sheet in which the other two sheets are represented by boxes as shown in Fig. A.1 and Fig. A.4.

Notice in Fig. A.3 and Fig. A.6 that sheet 3 (the LoRa ${ }^{\mathrm{TM}}$ transceiver) is the same in the full as well as the reduced PCB except for the values of the matching network components.

Sheet 2 in both designs contain some mechanical components (Epoxy Keepout) which are included in the schematic only for completing the exported BOM. This can be seen in Fig. A.2 and Fig. A.5. 


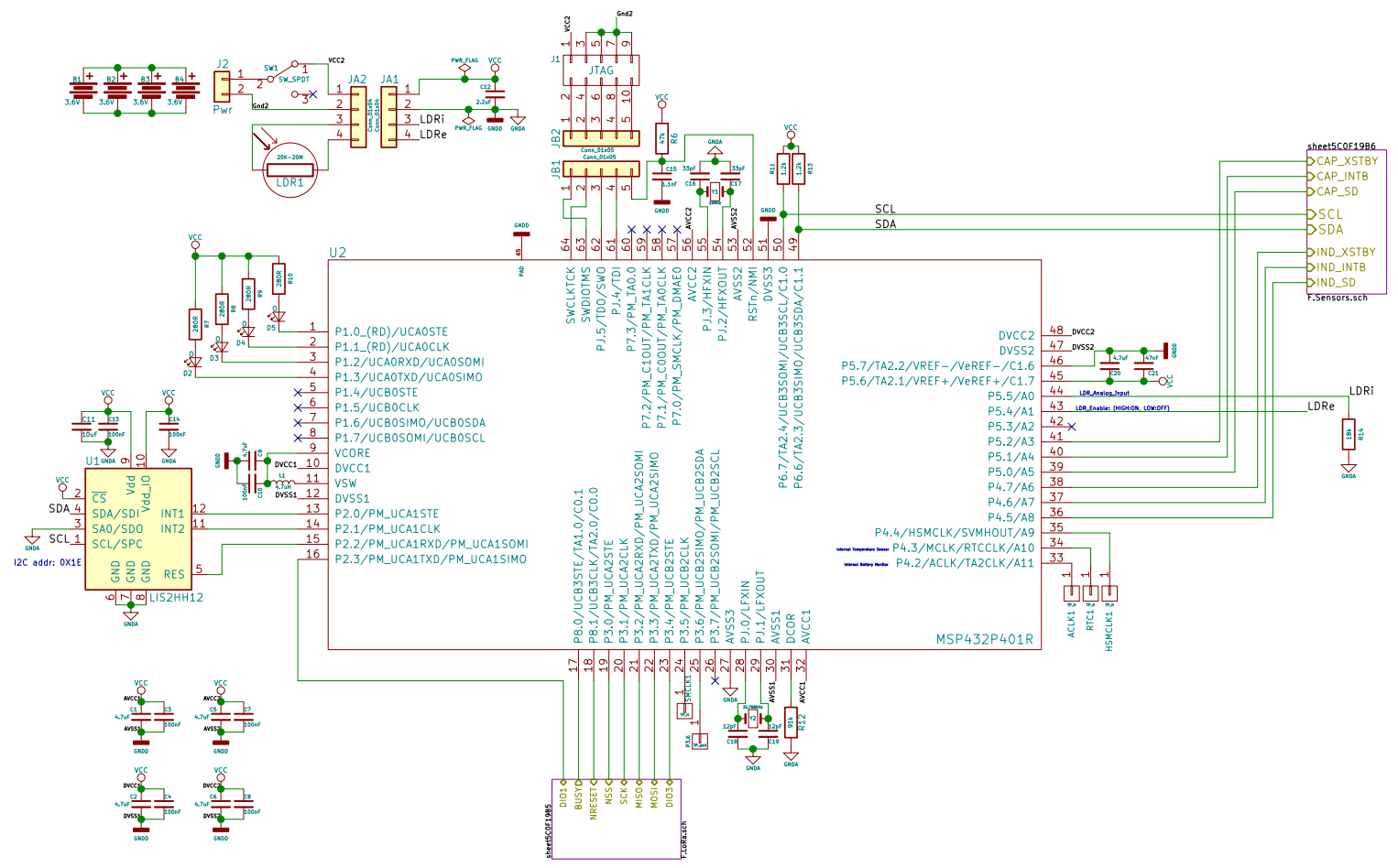

Figure A.1: Schematic diagram of the full featured PCB (Sheet 1). 


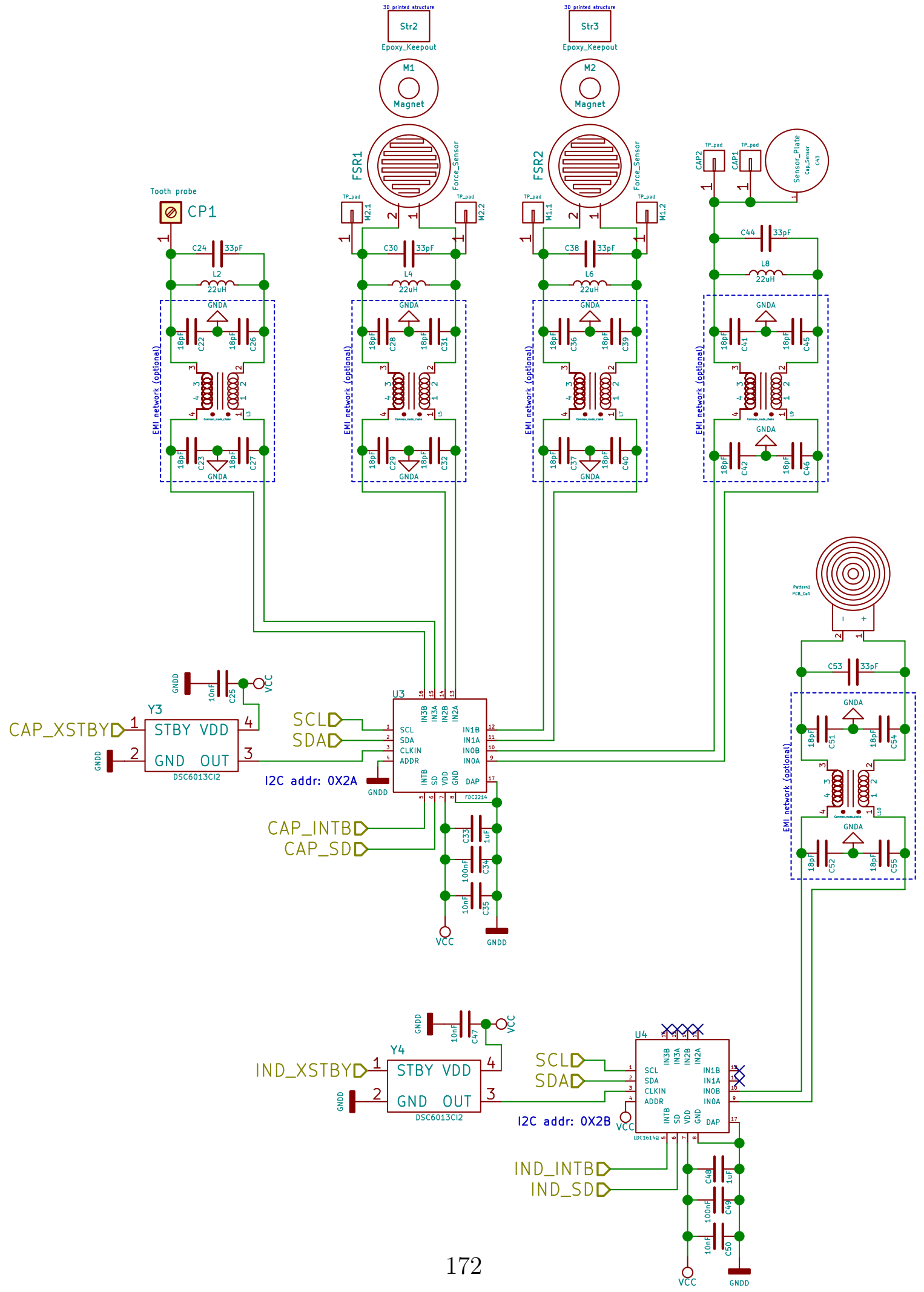

Figure A.2: Schematic diagram of the full featured PCB (Sheet 2). 


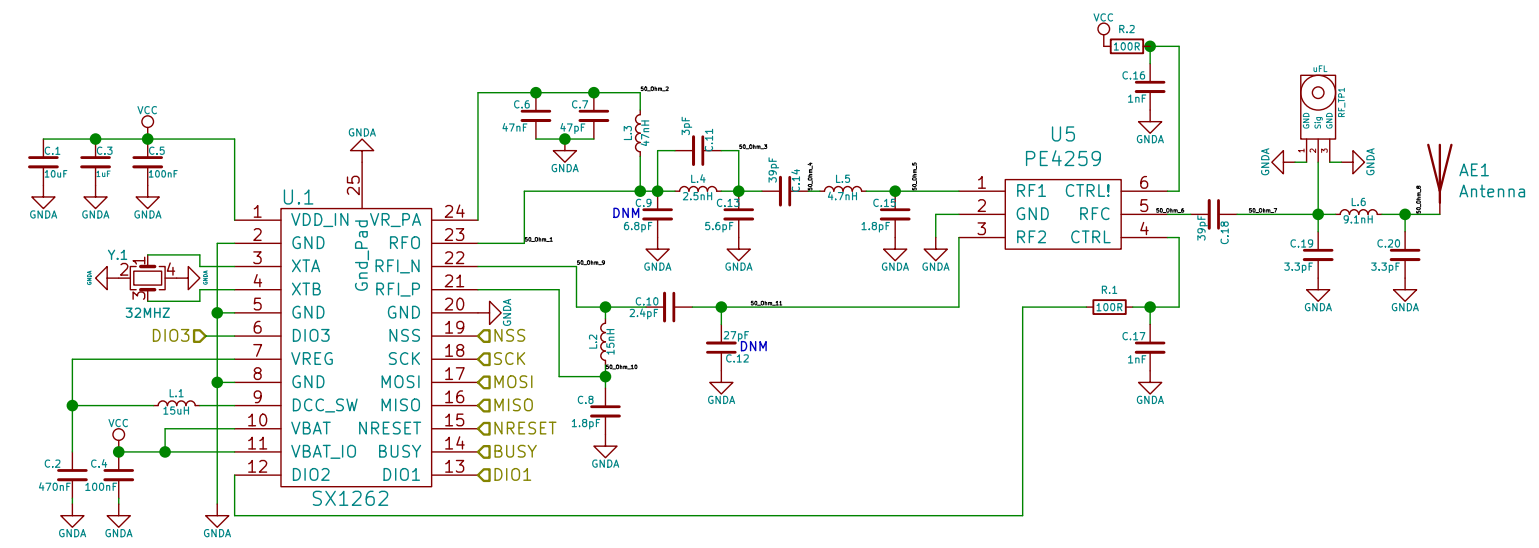

Figure A.3: Schematic diagram of the full featured PCB (Sheet 3).

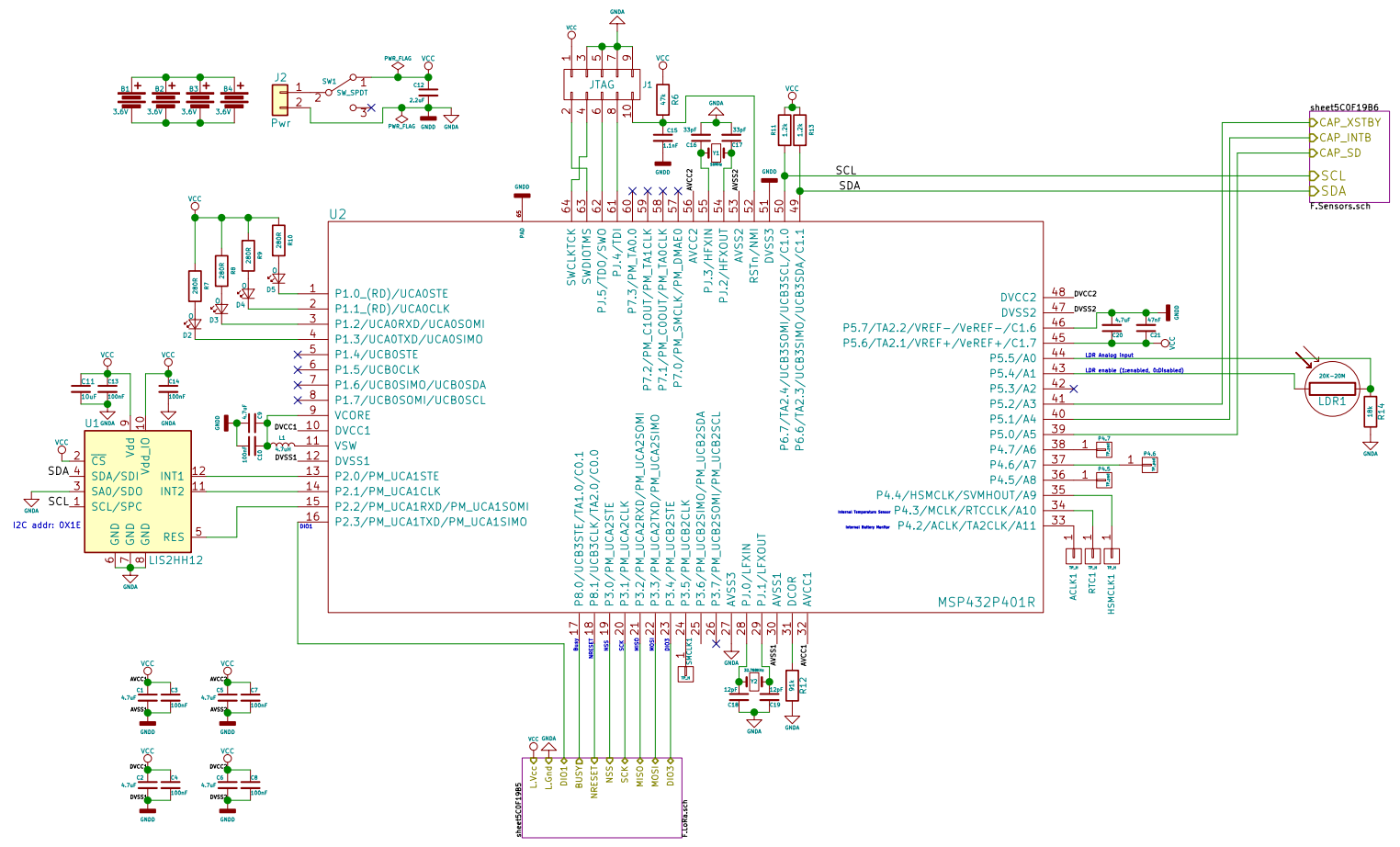

Figure A.4: Schematic diagram of the reduced PCB (Sheet 1). 


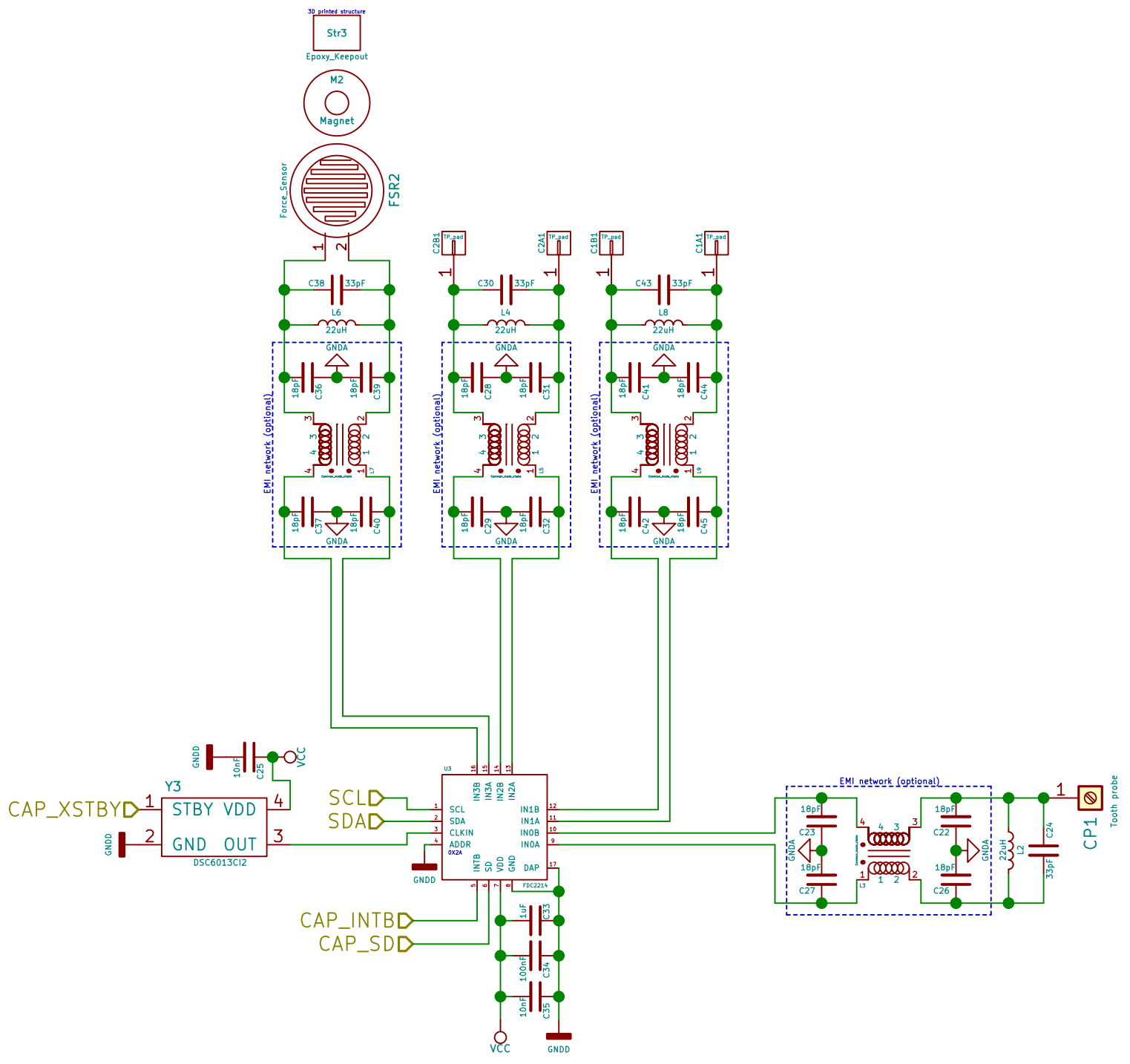

Figure A.5: Schematic diagram of the reduced PCB (Sheet 2). 


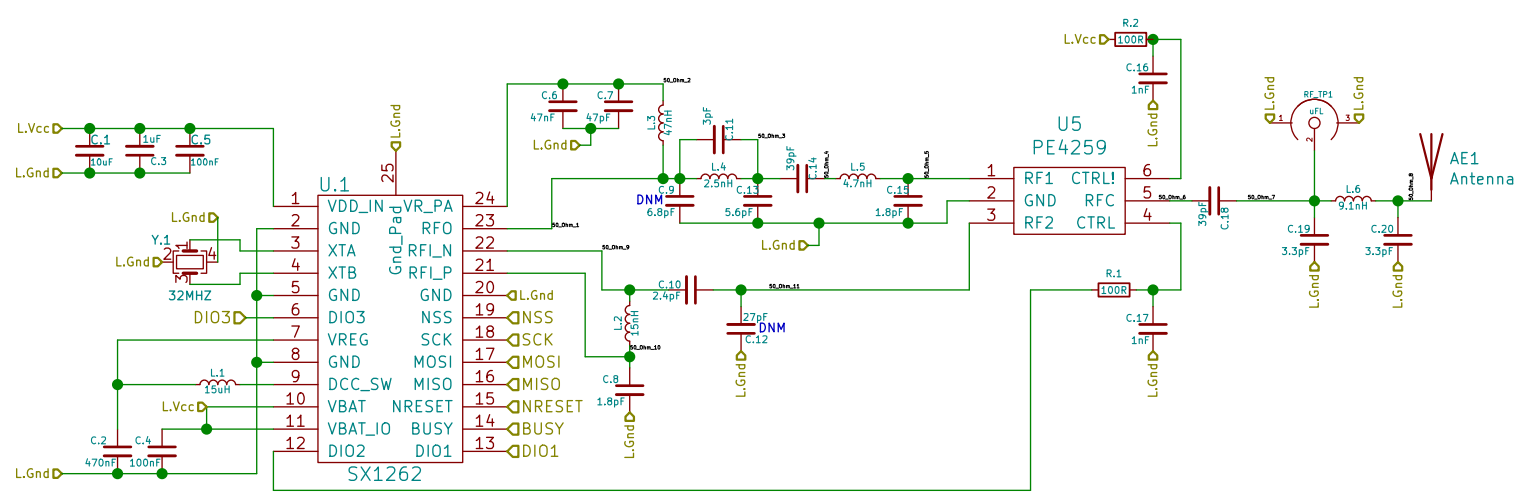

Figure A.6: Schematic diagram of the reduced PCB (Sheet 3). 


\section{Appendix B}

\section{Entropic Sensing Plots}

\section{B.1 Entropic Filtering}

This section shows each of the benchmarking signals going through the Entropic Filter (EF) stage. Notice in Fig. B.1 to Fig. B.11, how signals exhibit similar trends in their $|d H(X)|_{t}$. This confirms our earlier intuition that most samples carry little information while fewer samples carry most of the information. 

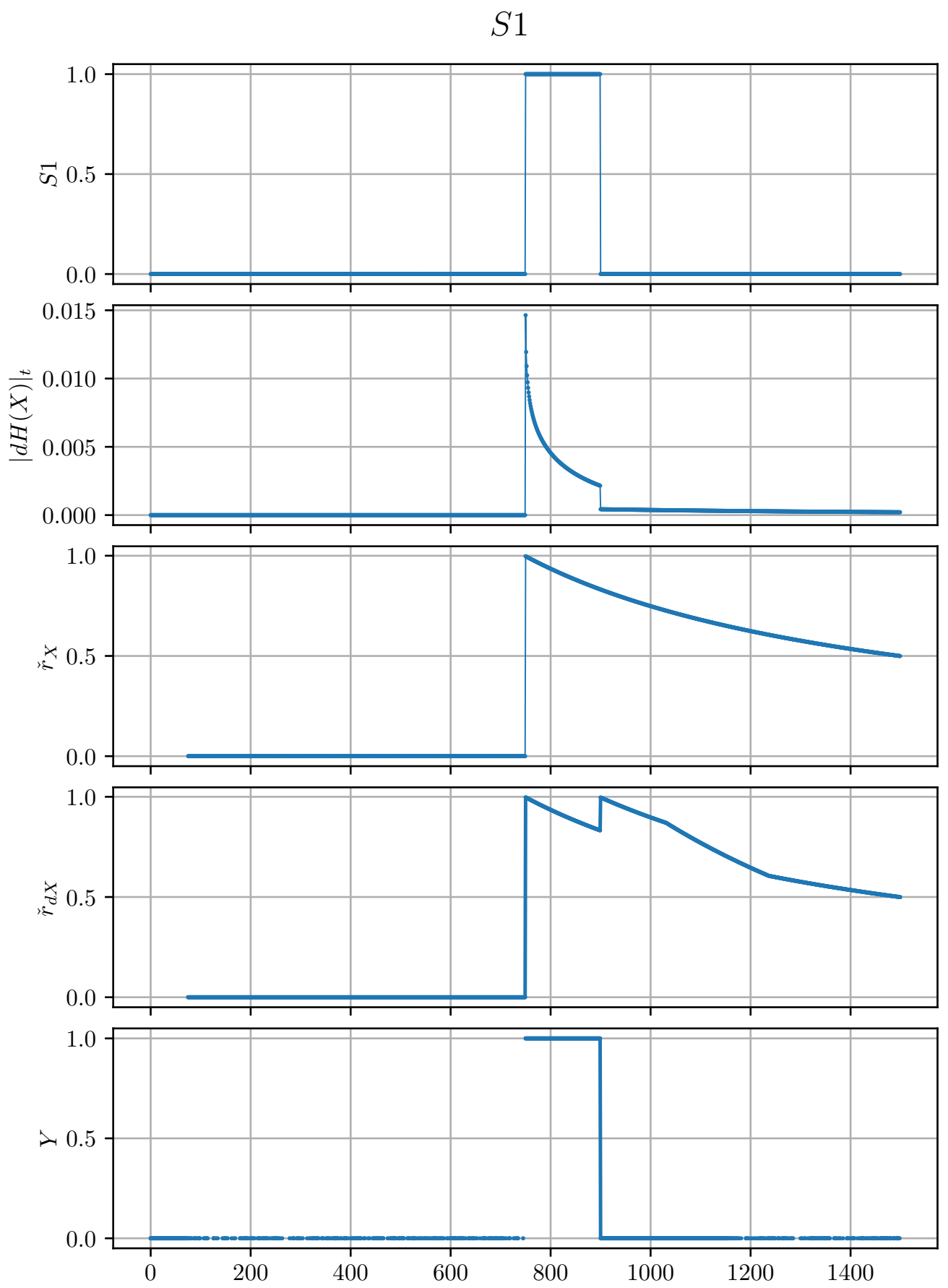

Figure B.1: Entropic filtering steps for $S 1$. 
$S 2$
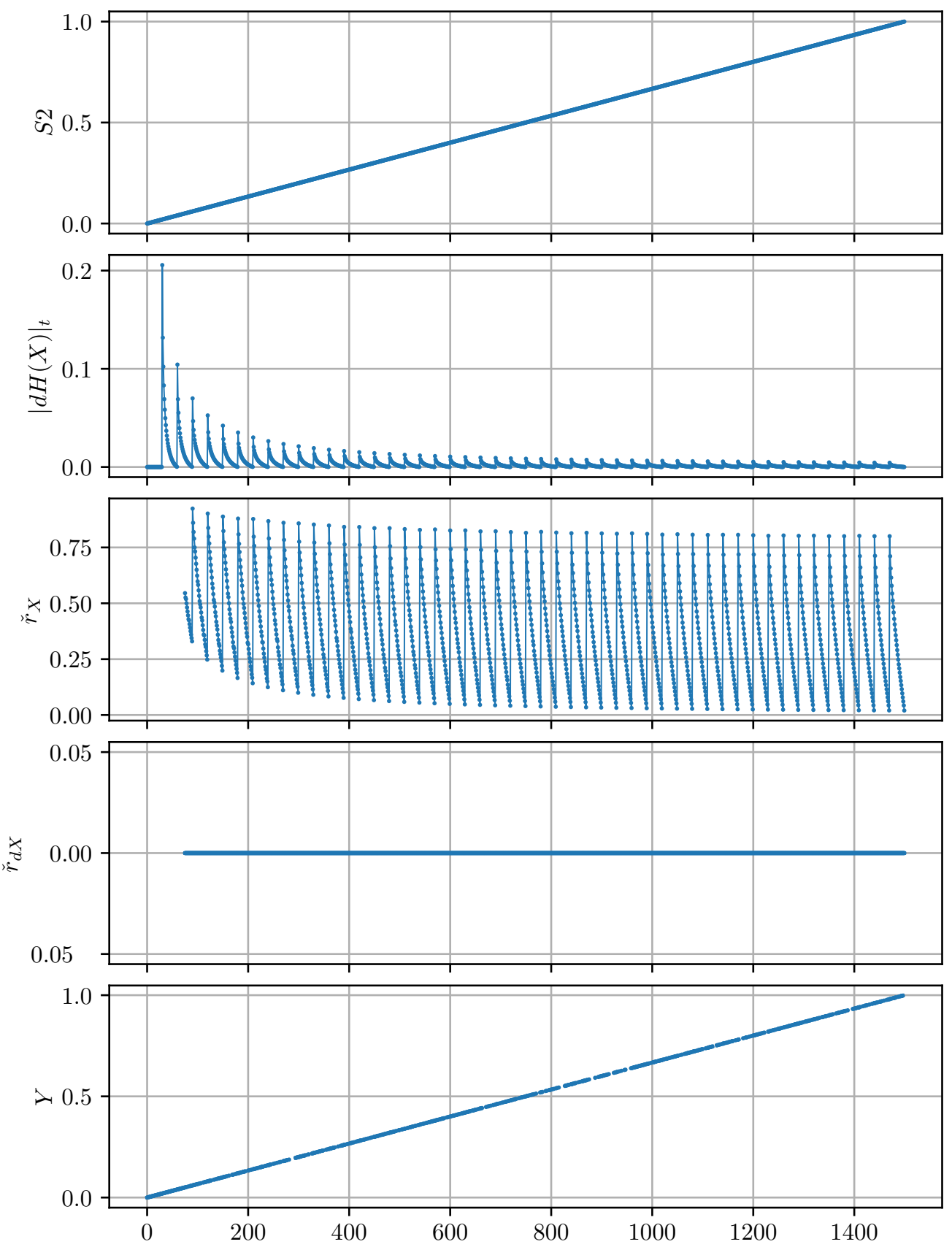

Figure B.2: Entropic filtering steps for $S 2$. 

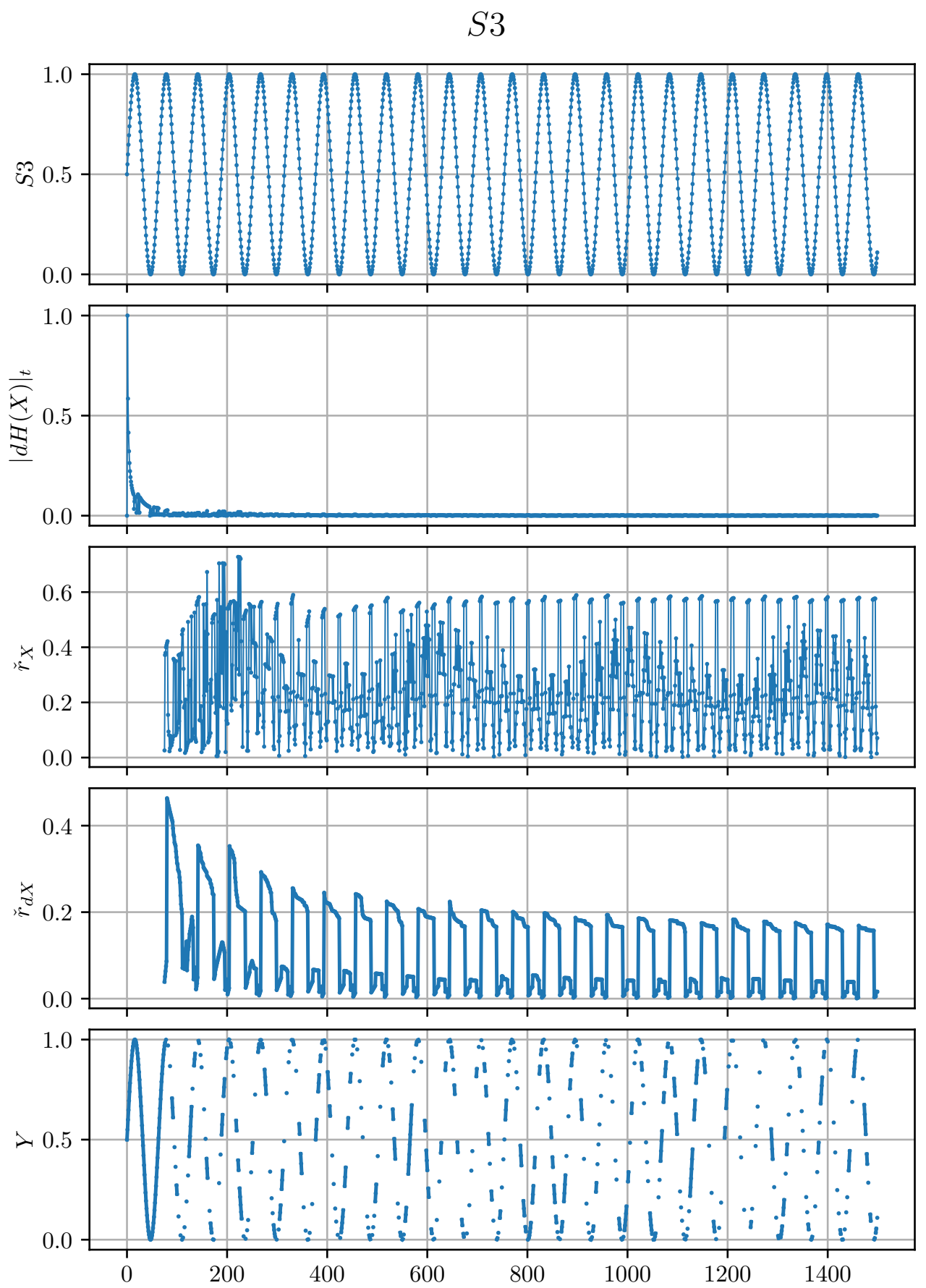

Figure B.3: Entropic filtering steps for $S 3$. 

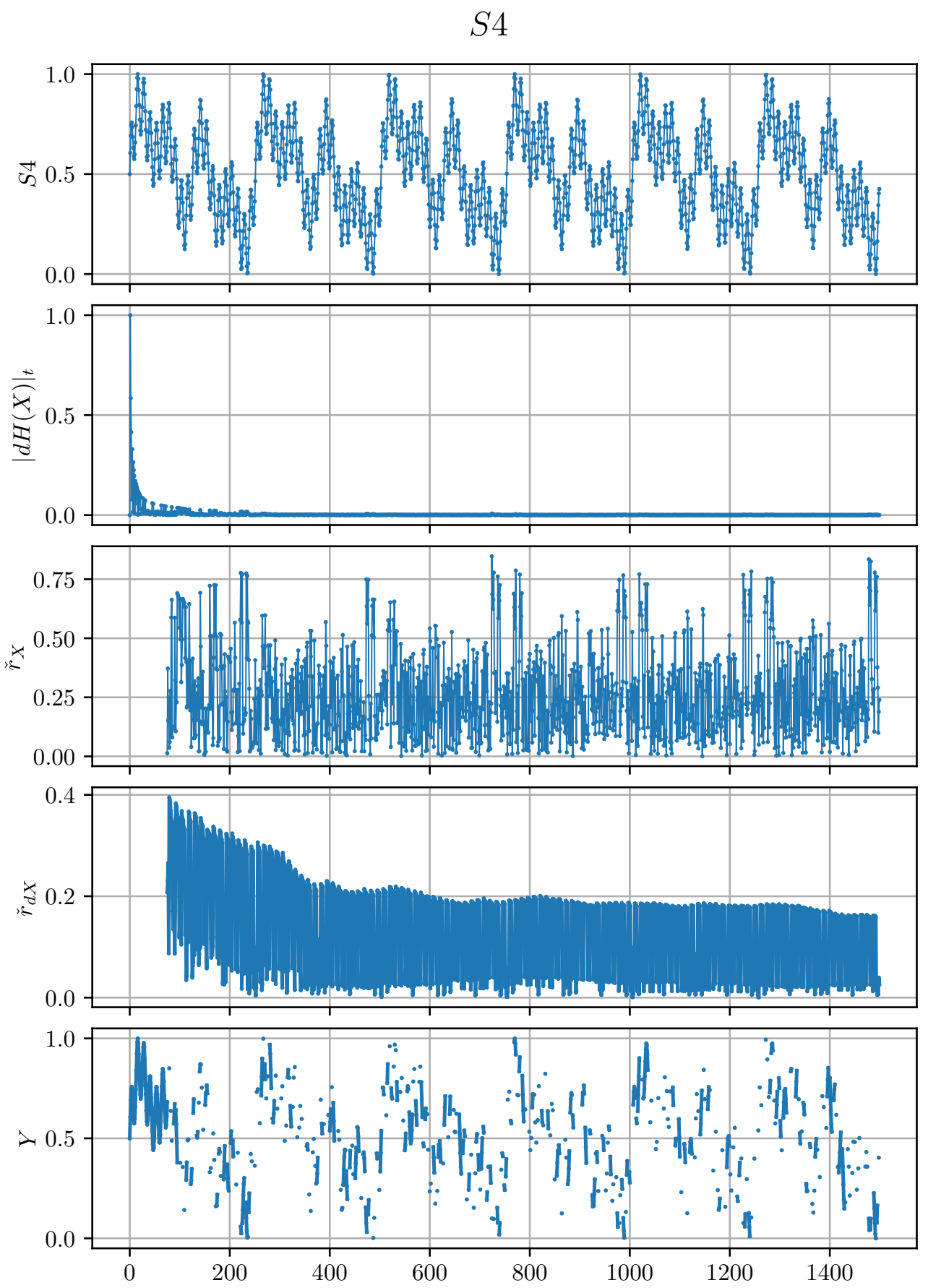

Figure B.4: Entropic filtering steps for $S 4$. 

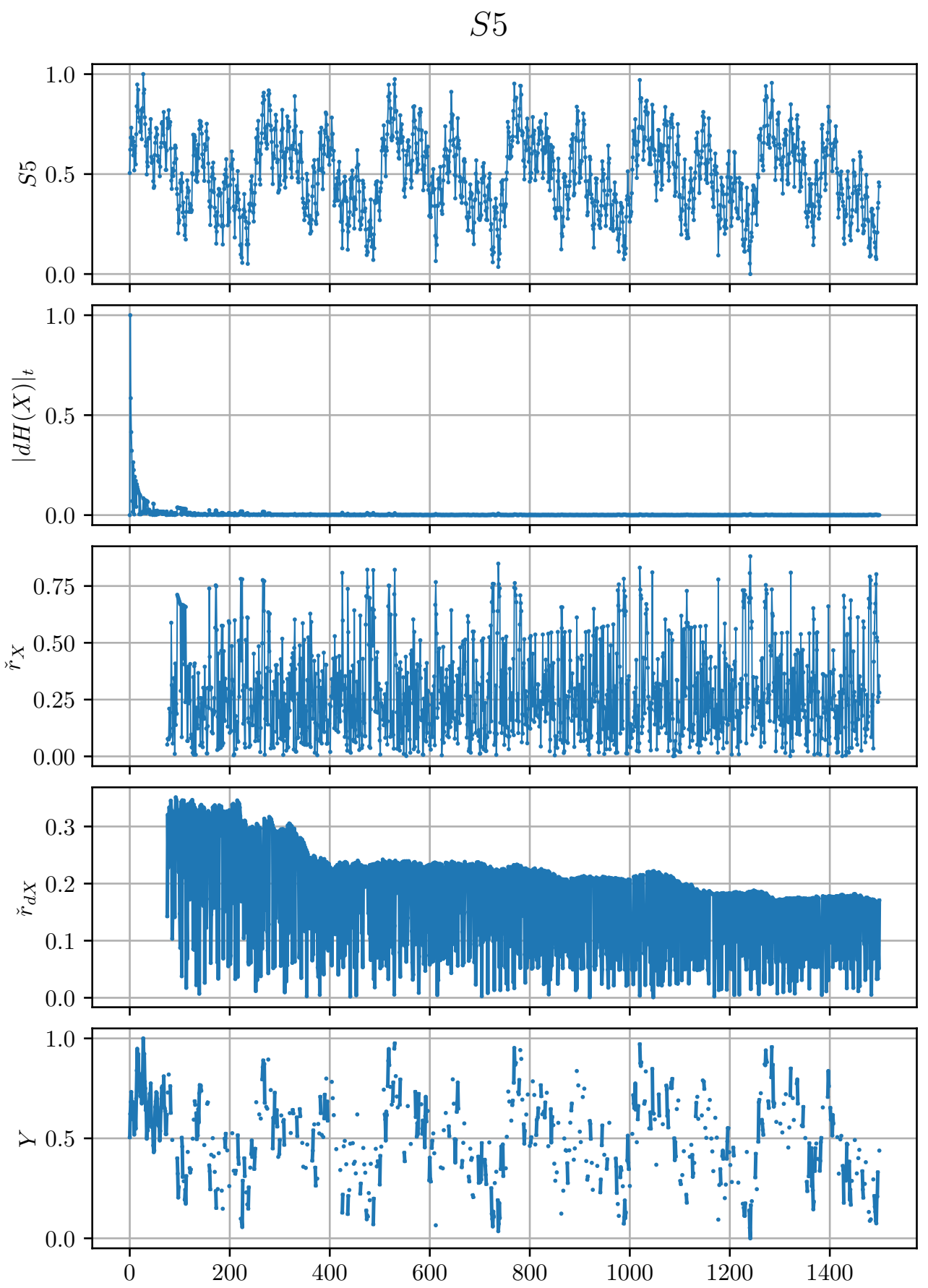

Figure B.5: Entropic filtering steps for $S 5$. 

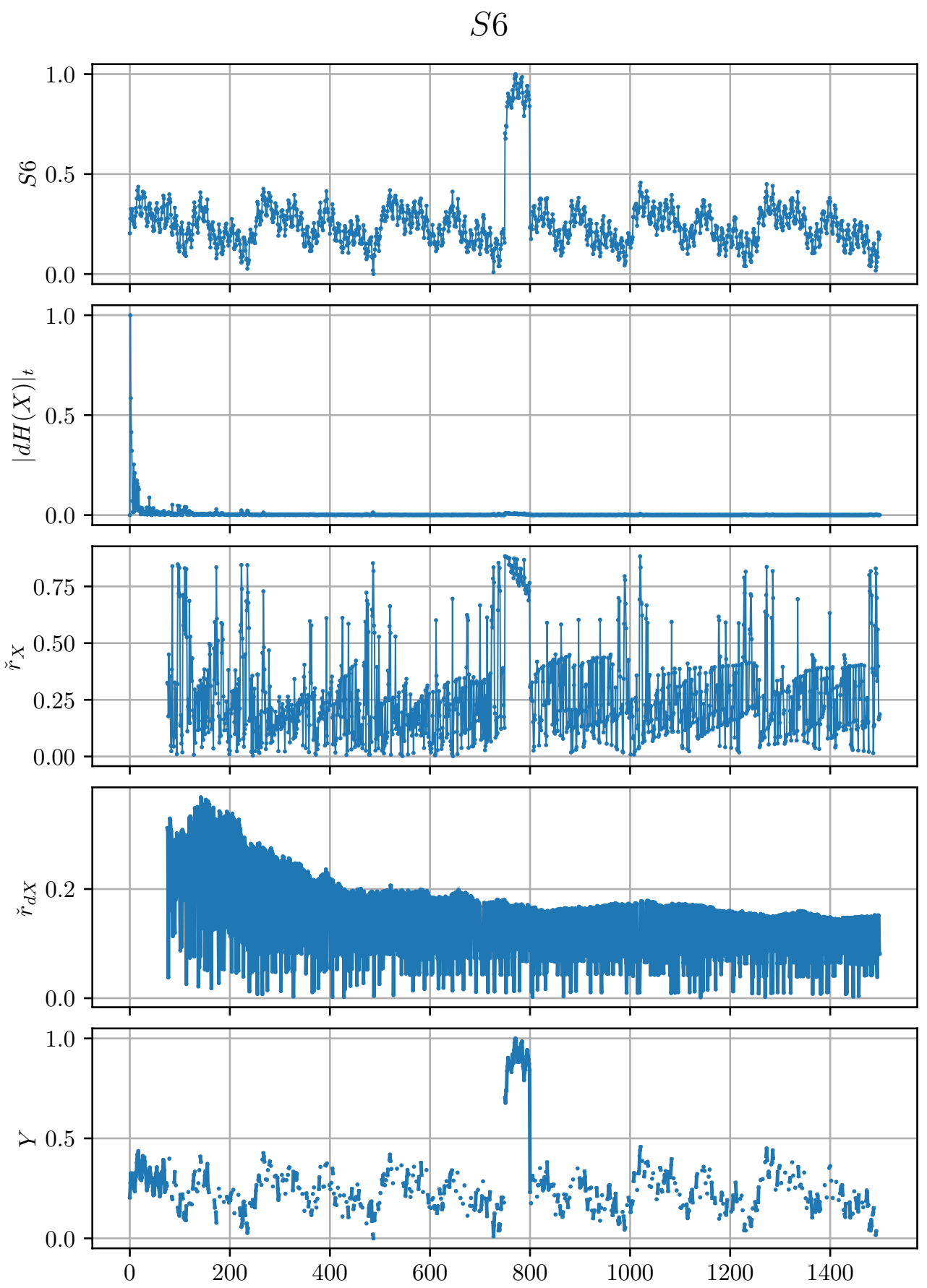

Figure B.6: Entropic filtering steps for $S 6$. 

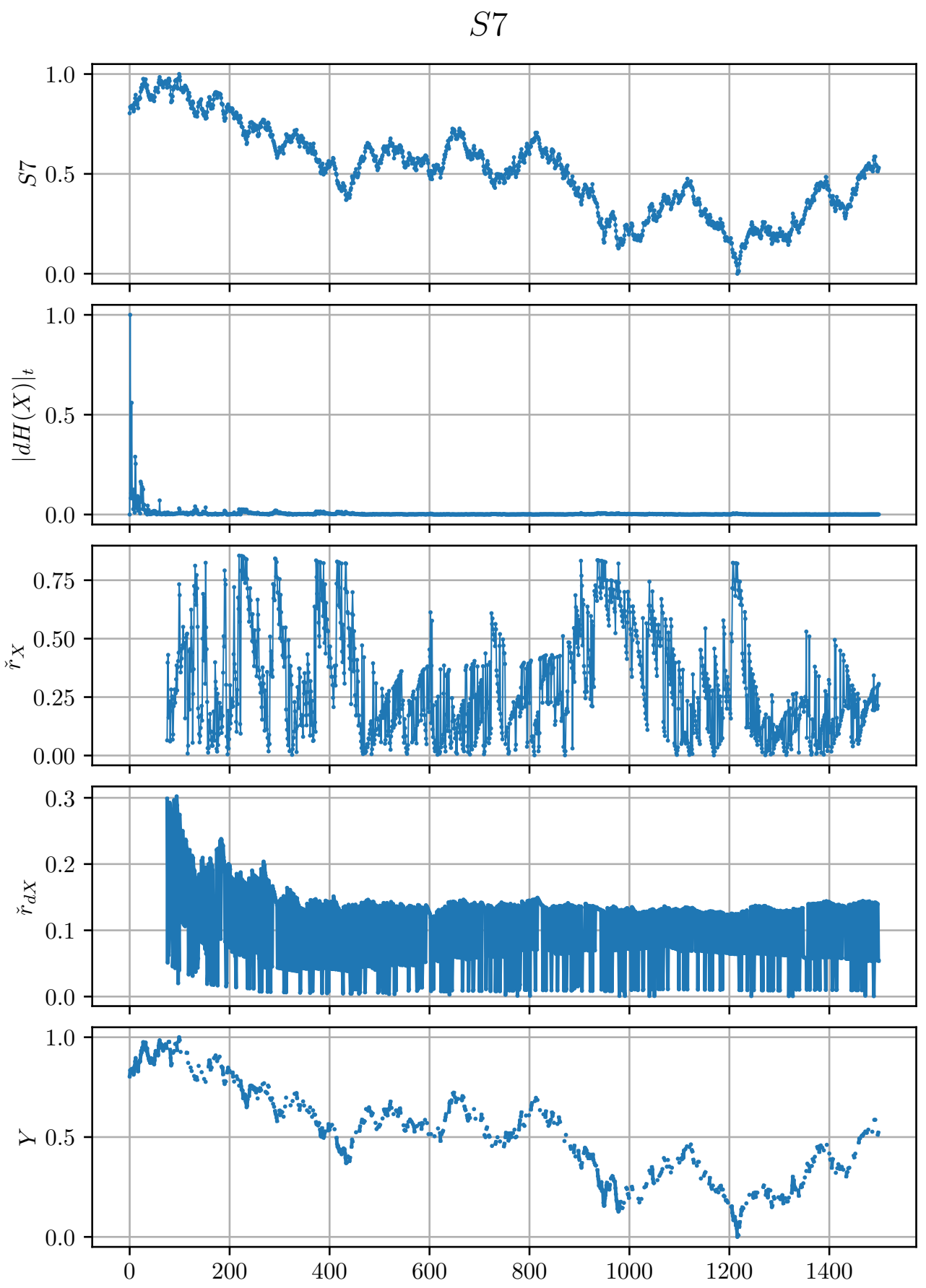

Figure B.7: Entropic filtering steps for $S 7$. 

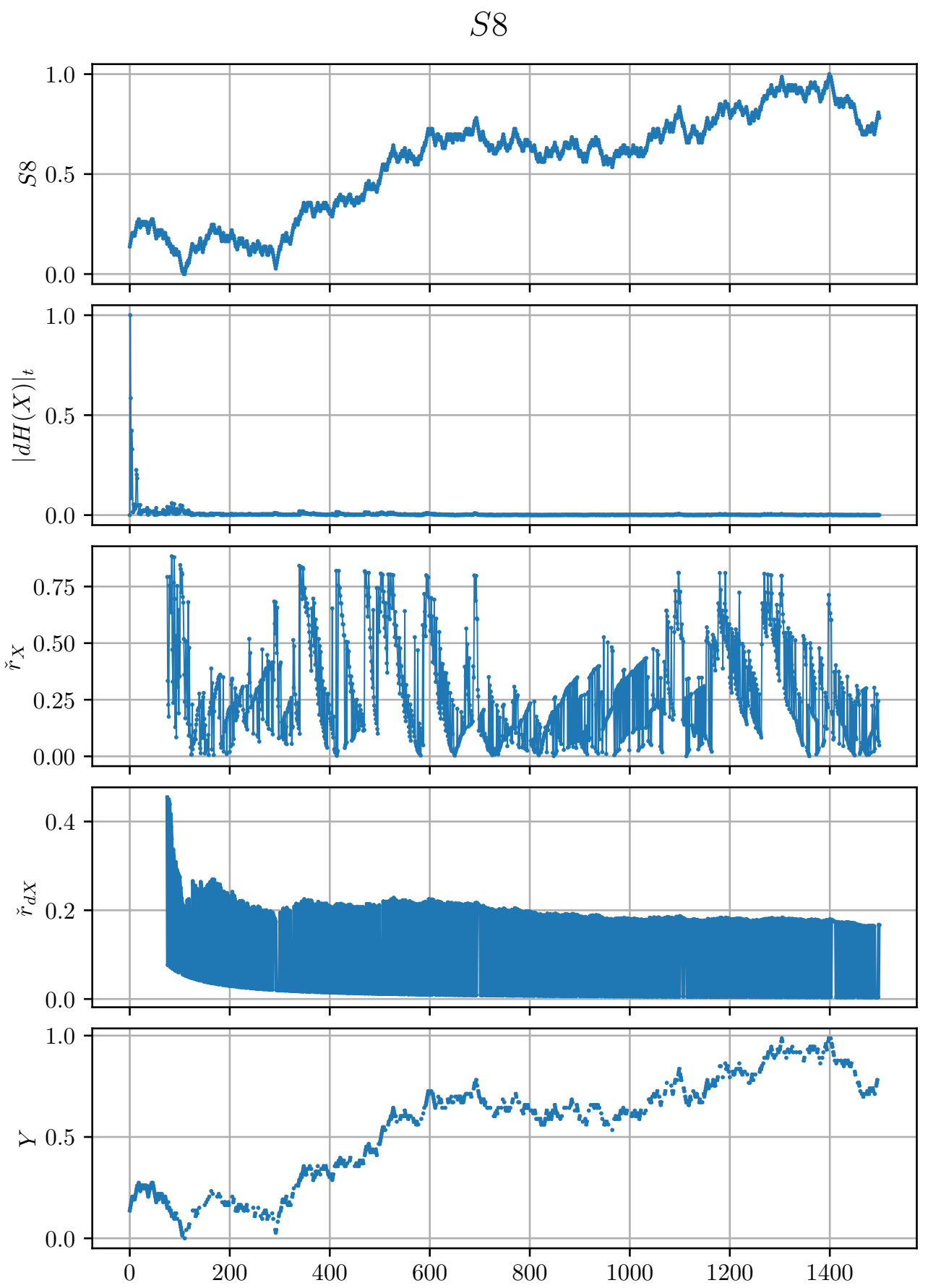

Figure B.8: Entropic filtering steps for $S 8$. 

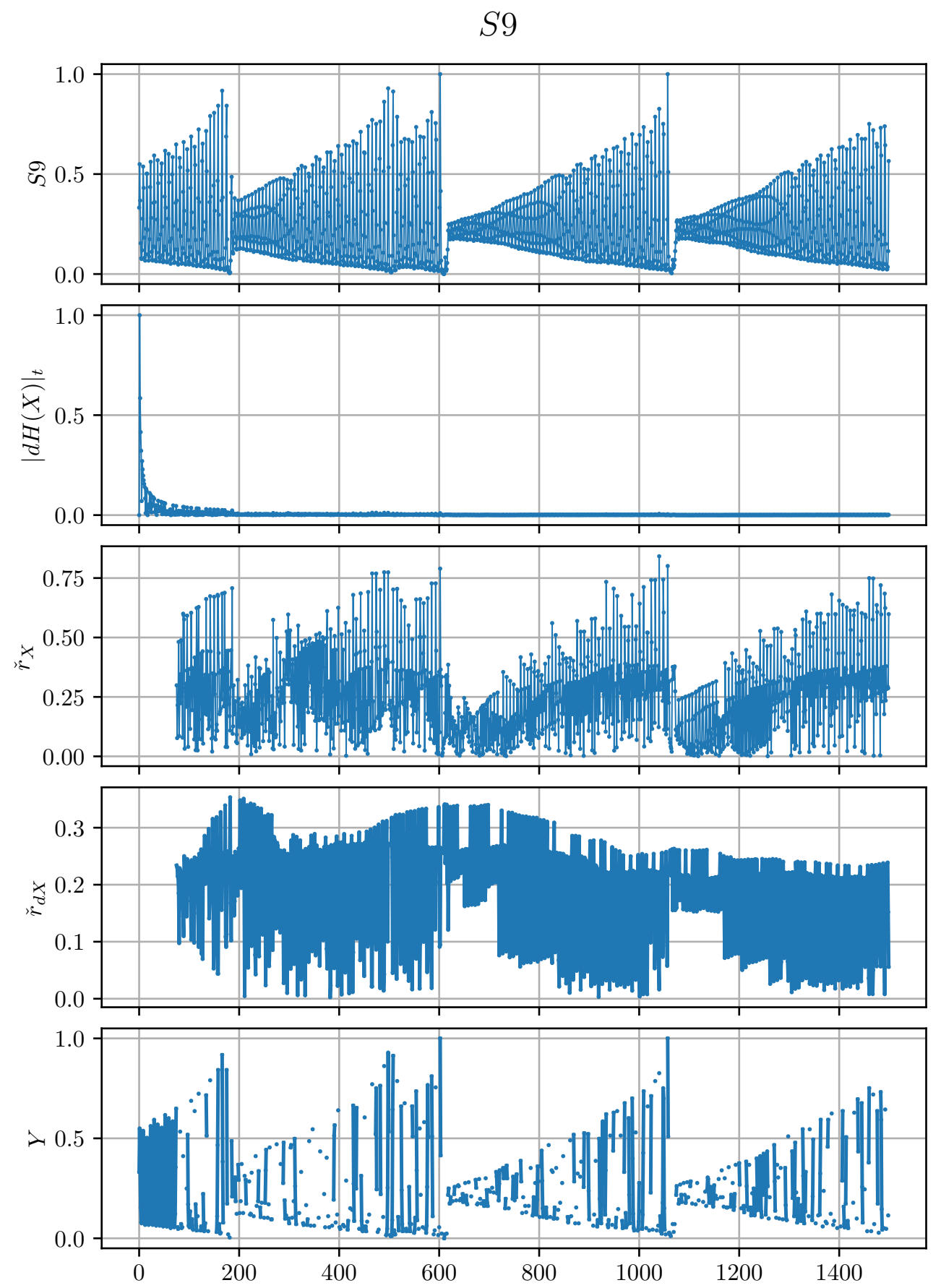

Figure B.9: Entropic filtering steps for $S 9$. 
$S 10$
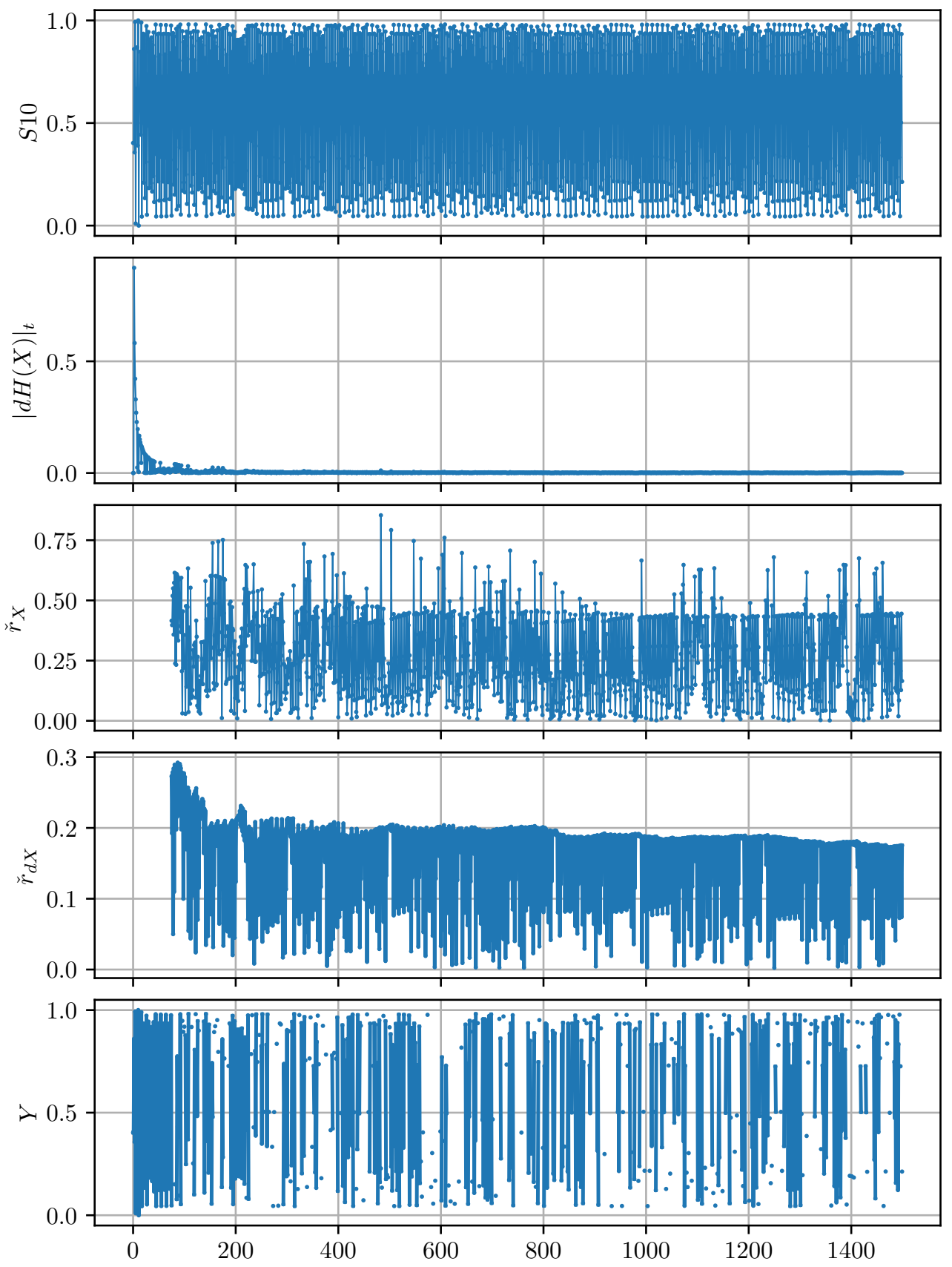

Figure B.10: Entropic filtering steps for $S 10$. 

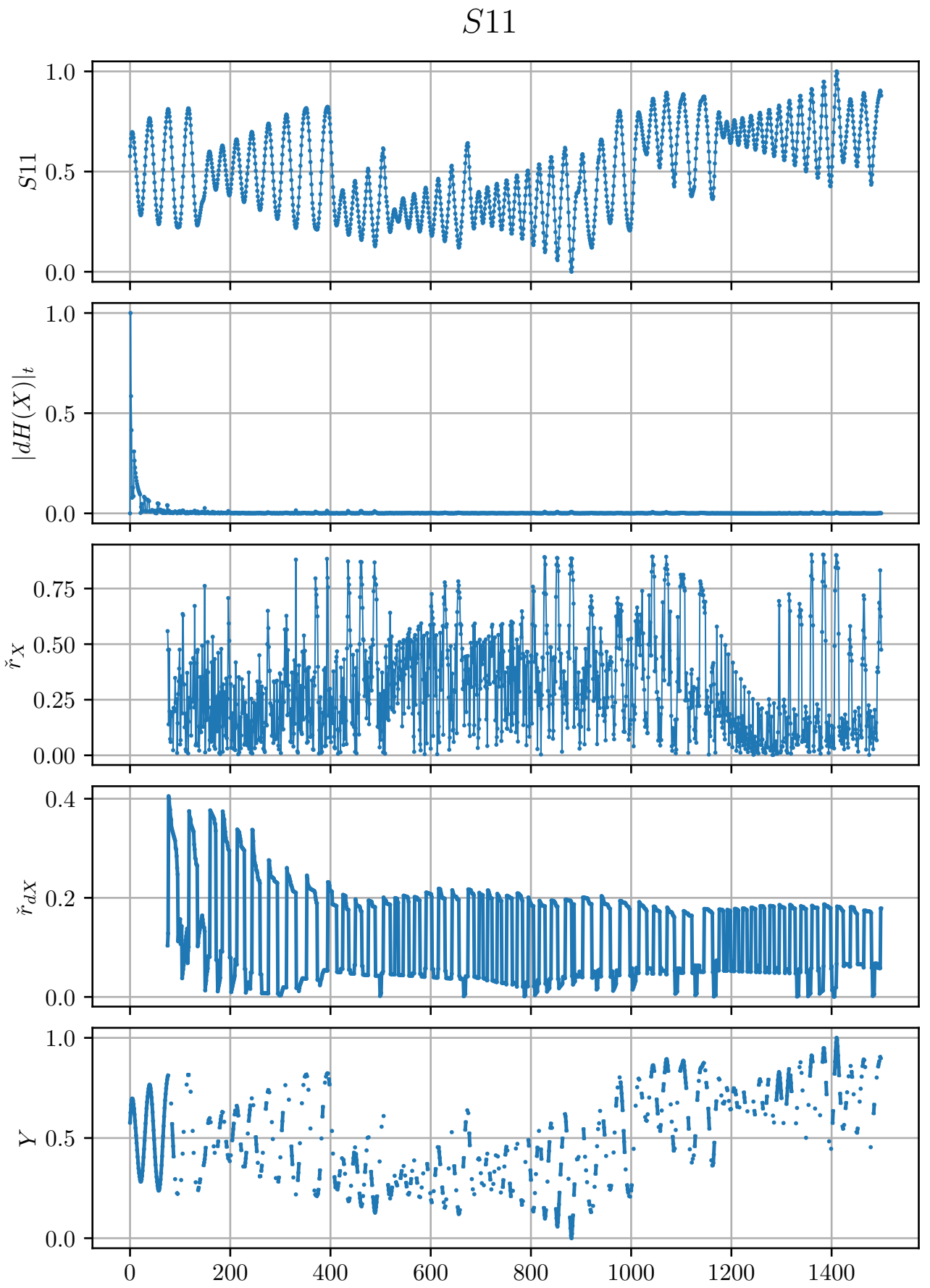

Figure B.11: Entropic filtering steps for $S 11$. 


\section{B.2 Computing Entropy}

This section demonstrates how Instantaneous Entropy (IE) progresses in comparison with the given benchmarking signal. Notice how the IE saturates quickly to a stable level with non-random signals as shown in Fig. B.14 to Fig. B.17 and Fig. B.20 to Fig. B.22, while with random signals in Fig. B.18 and Fig. B.19, the IE does not saturate due to the continuous addition of new information through time.

Moreover, the overlapping of the IE's calculations by Shannon's formula and the iterative formula in all signals demonstrates the exactness of the iterative formula. 

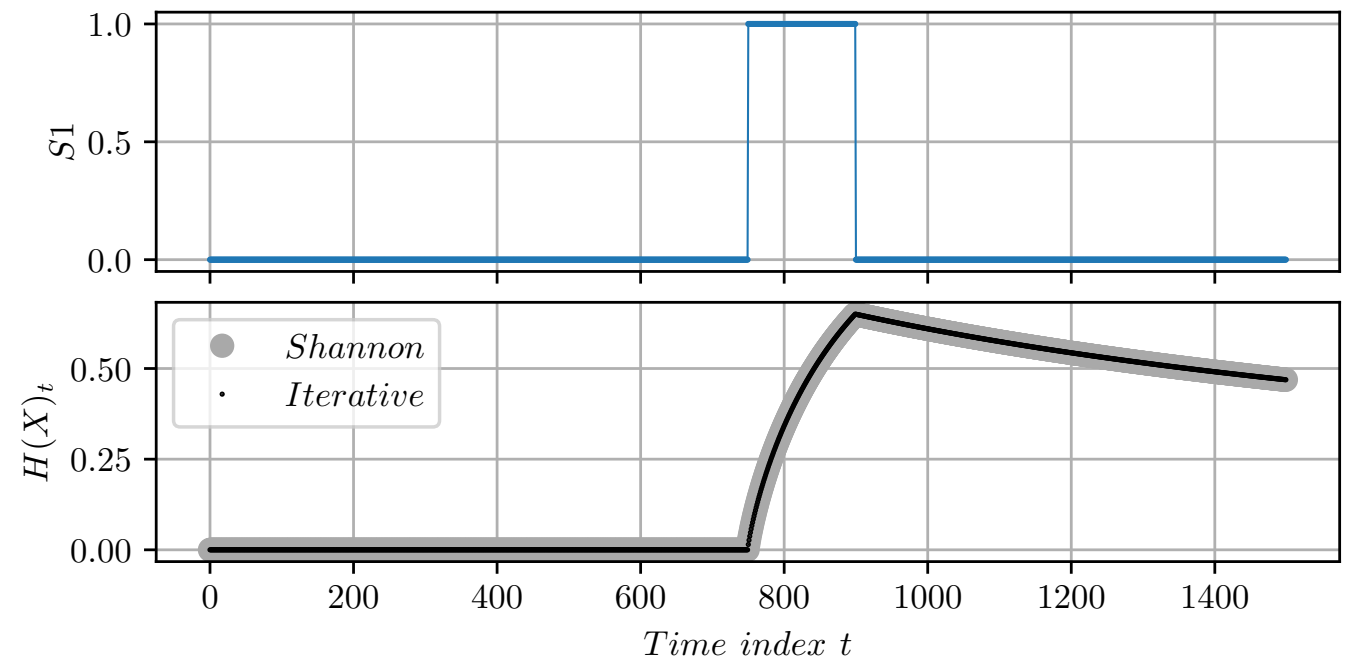

Figure B.12: $S 1$ and its instantaneous Entropy using Shannon's formula and its iterative form.
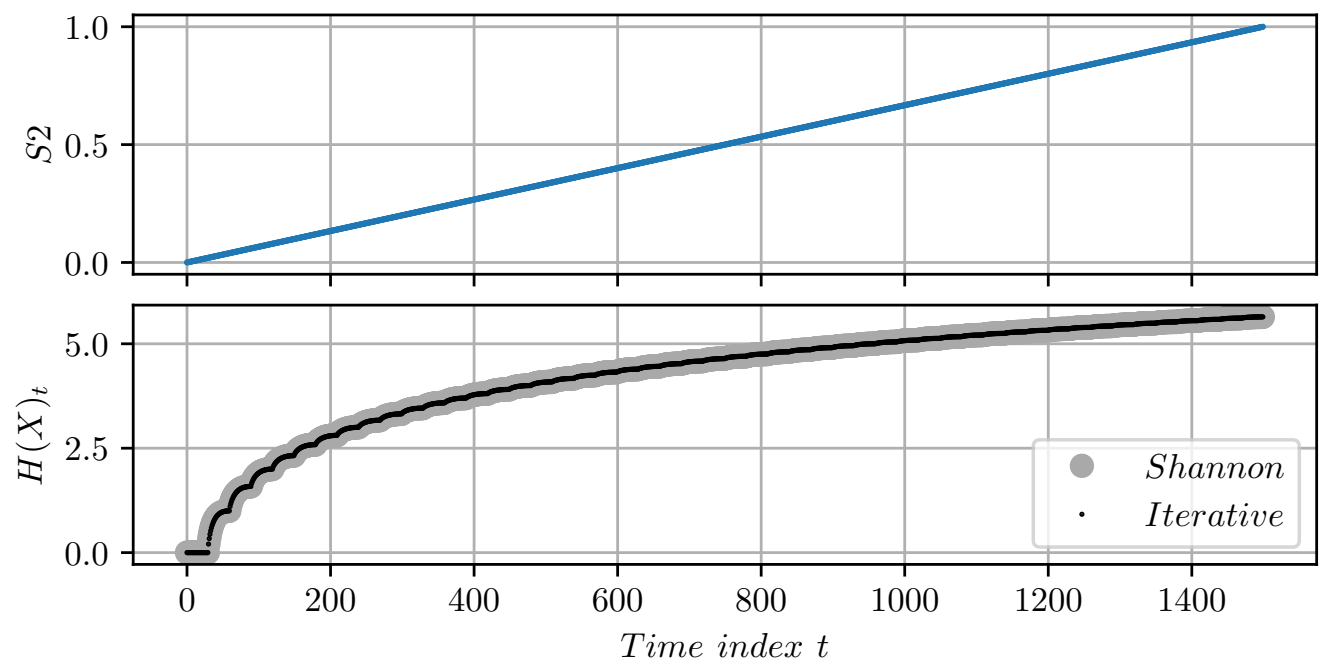

Figure B.13: $S 2$ and its instantaneous Entropy using Shannon's formula and its iterative form. 

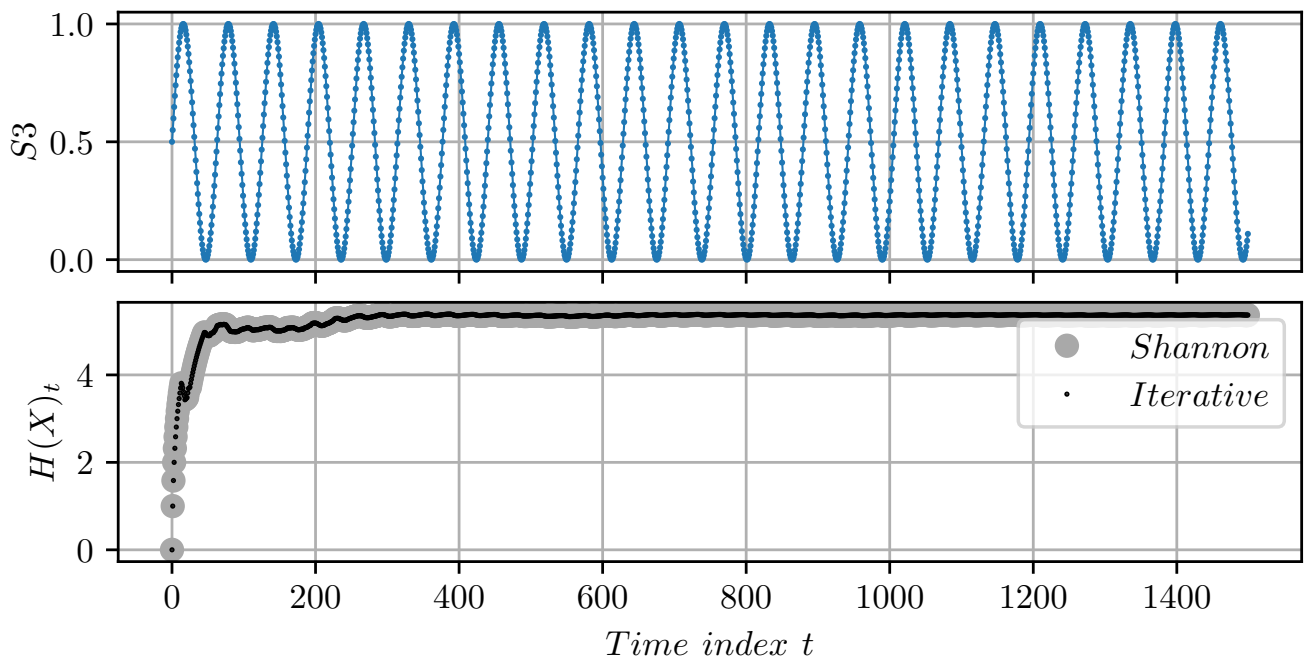

Figure B.14: $S 3$ and its instantaneous Entropy using Shannon's formula and its iterative form.
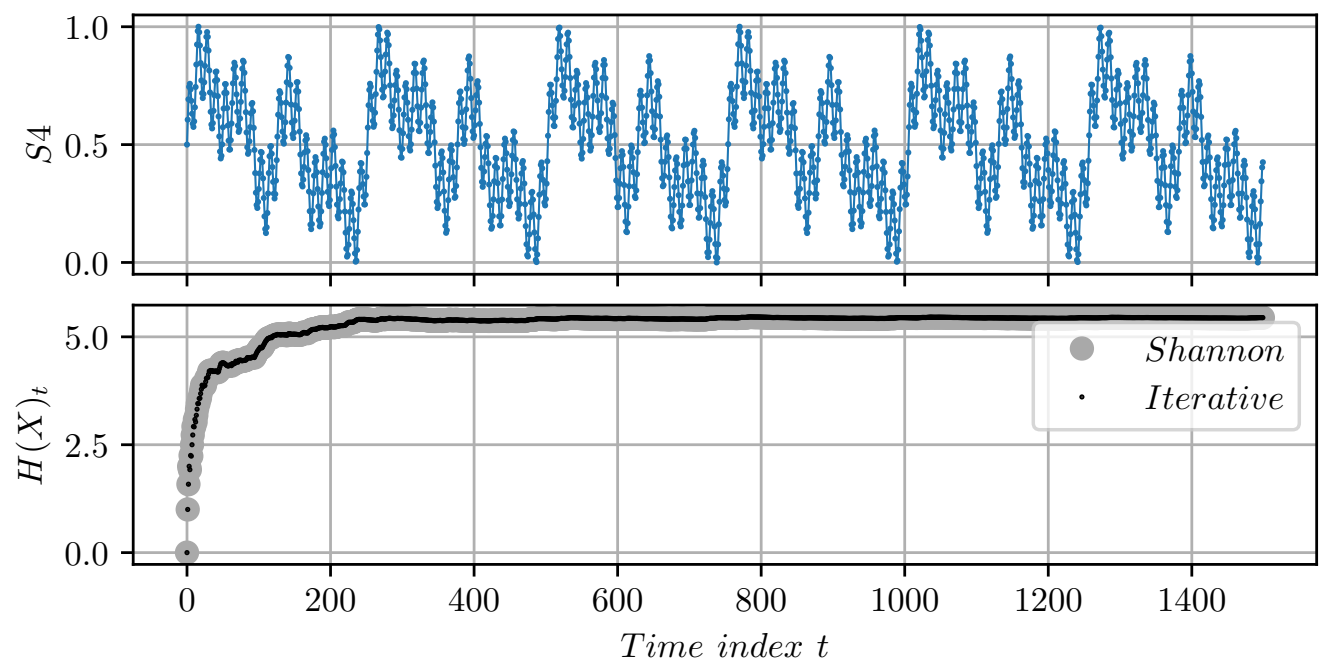

Figure B.15: $S 4$ and its instantaneous Entropy using Shannon's formula and its iterative form. 

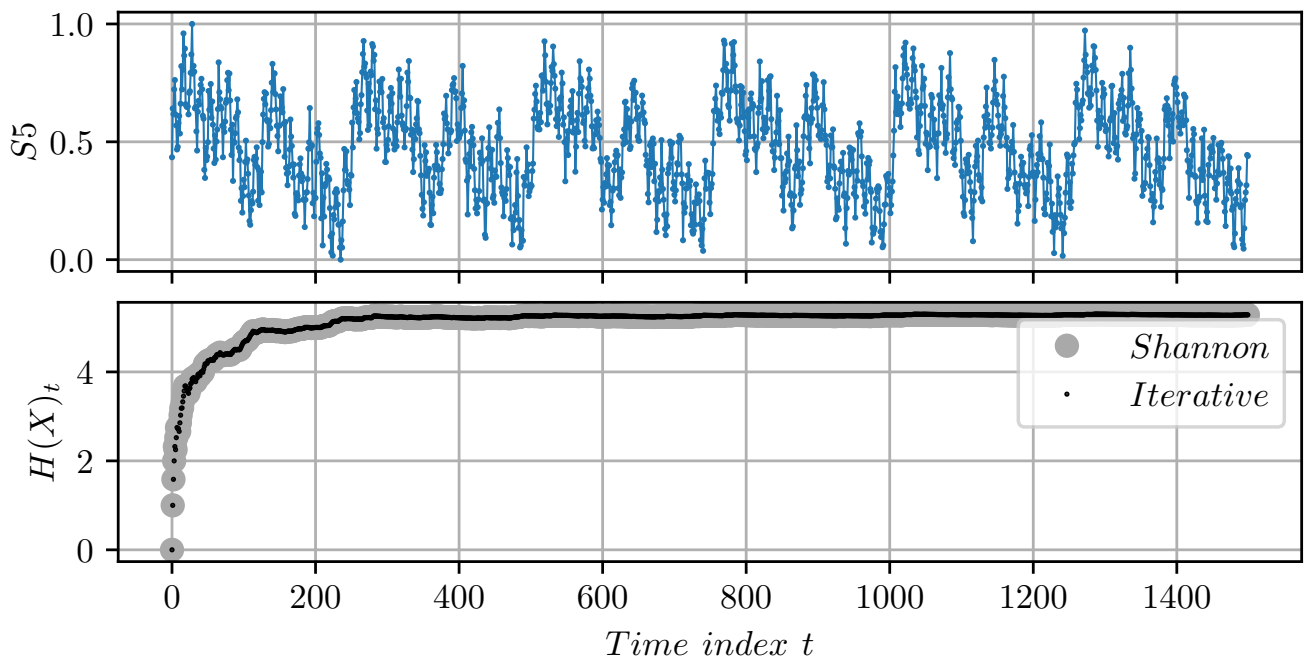

Figure B.16: $S 5$ and its instantaneous Entropy using Shannon's formula and its iterative form.
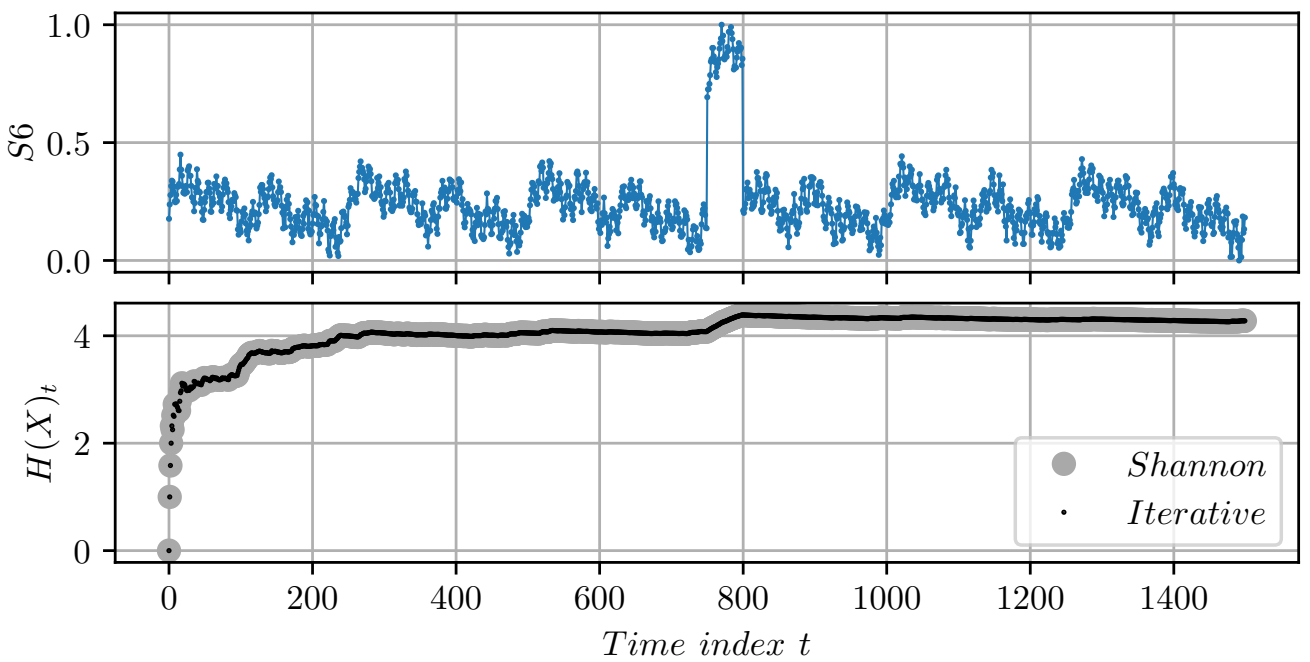

Figure B.17: $S 6$ and its instantaneous Entropy using Shannon's formula and its iterative form. 

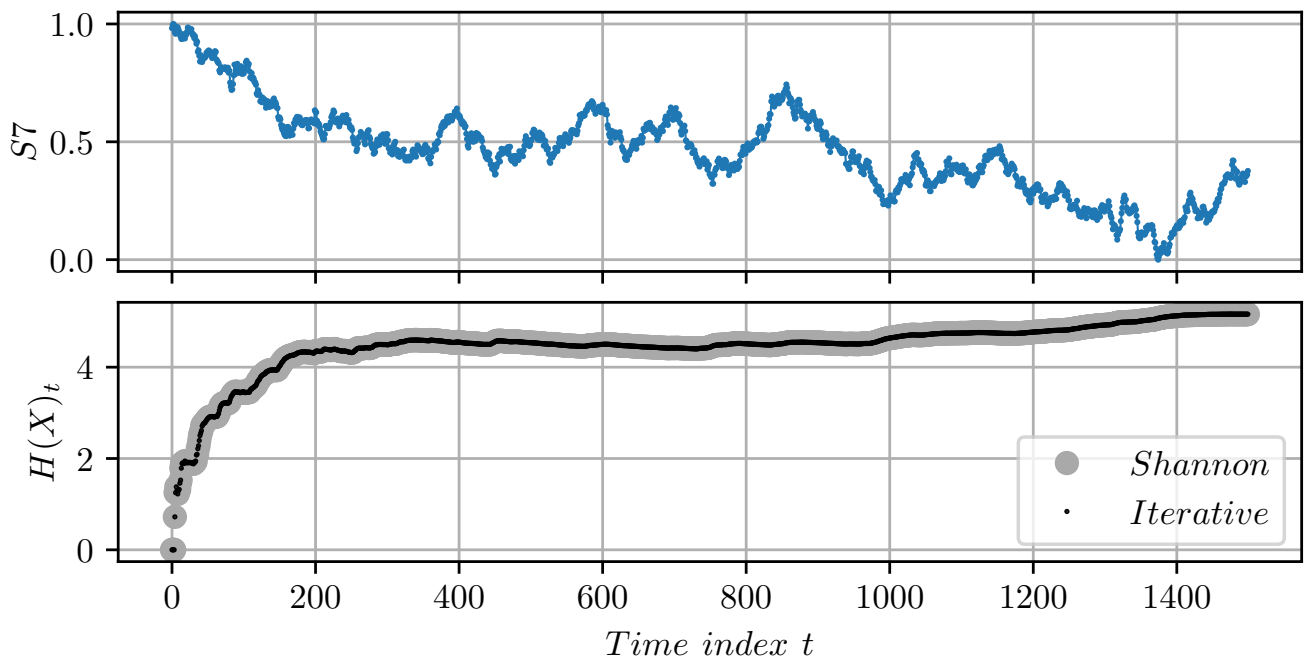

Figure B.18: $S 7$ and its instantaneous Entropy using Shannon's formula and its iterative form.
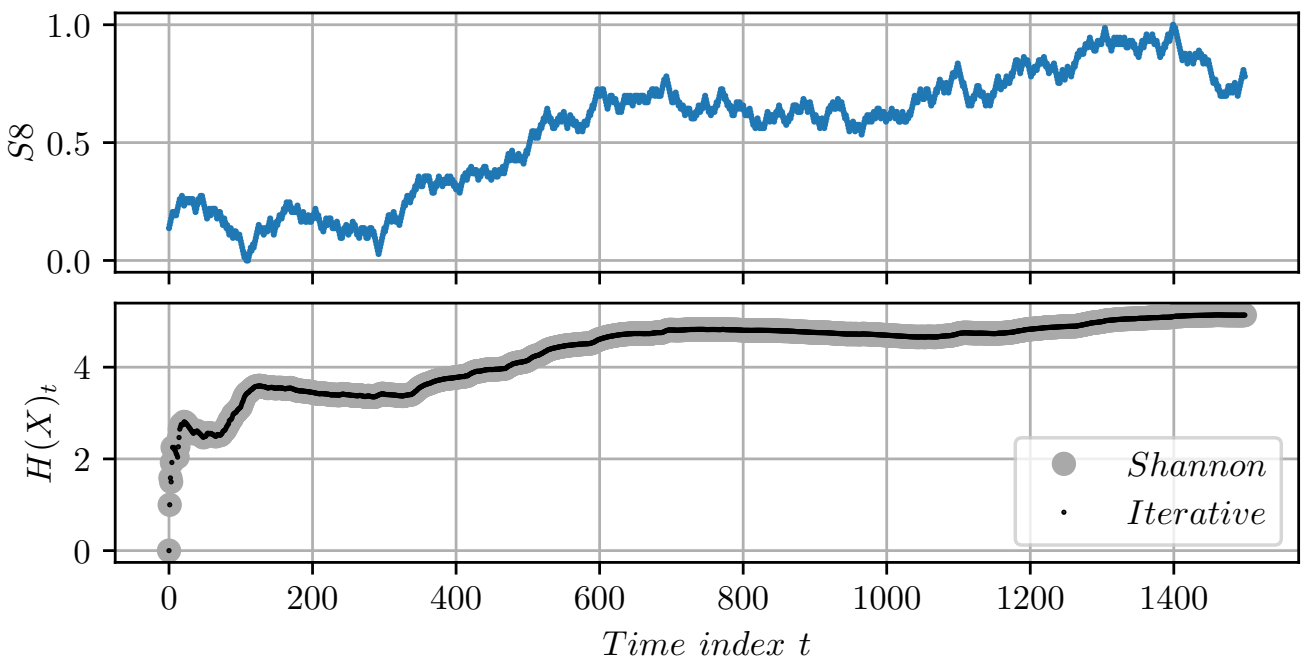

Figure B.19: $S 8$ and its instantaneous Entropy using Shannon's formula and its iterative form. 

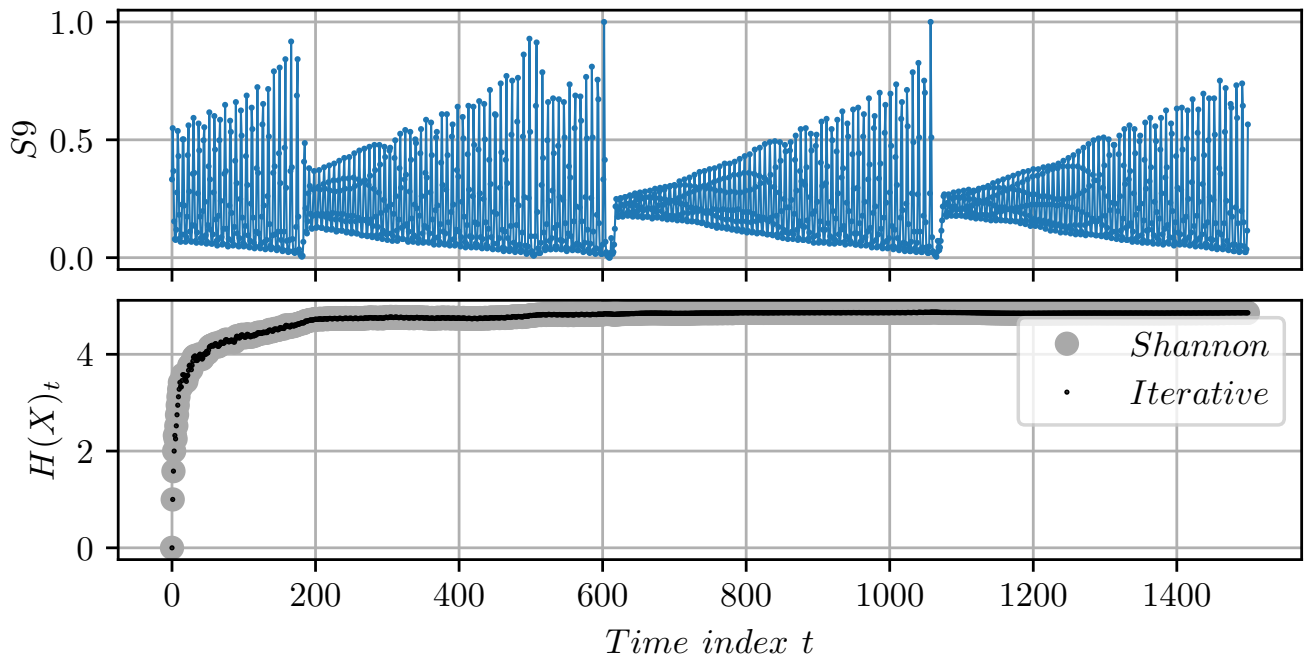

Figure B.20: $S 9$ and its instantaneous Entropy using Shannon's formula and its iterative form.
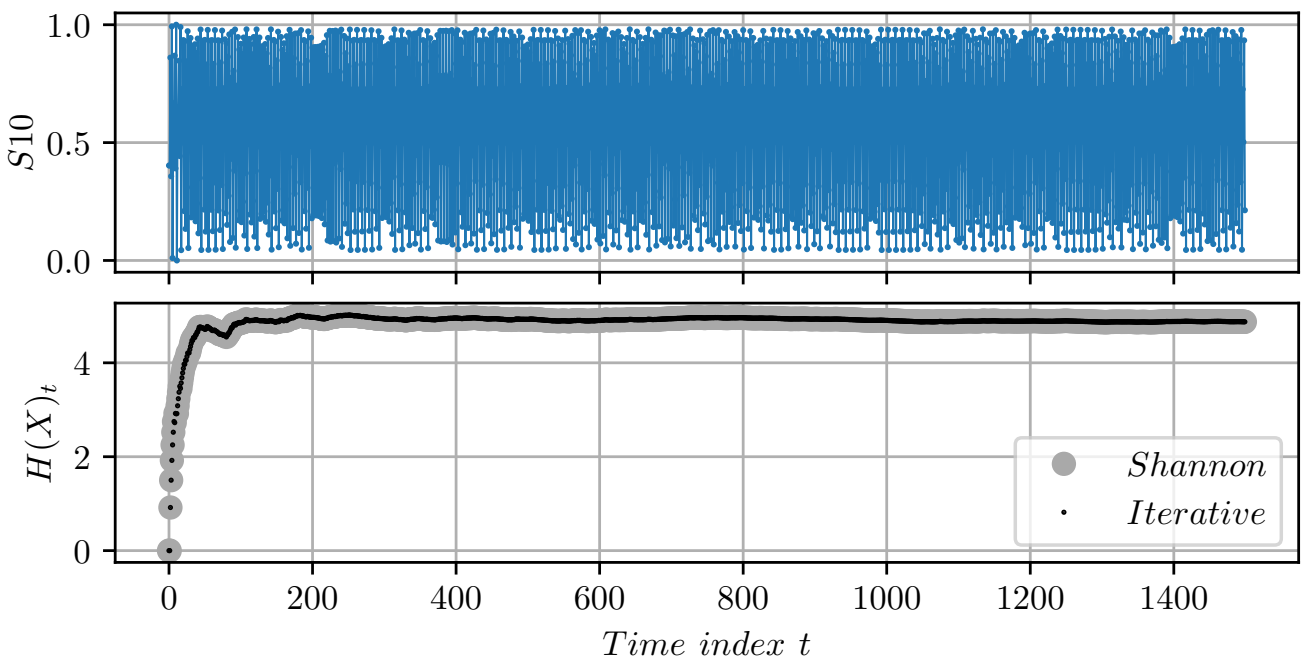

Figure B.21: $S 10$ and its instantaneous Entropy using Shannon's formula and its iterative form. 

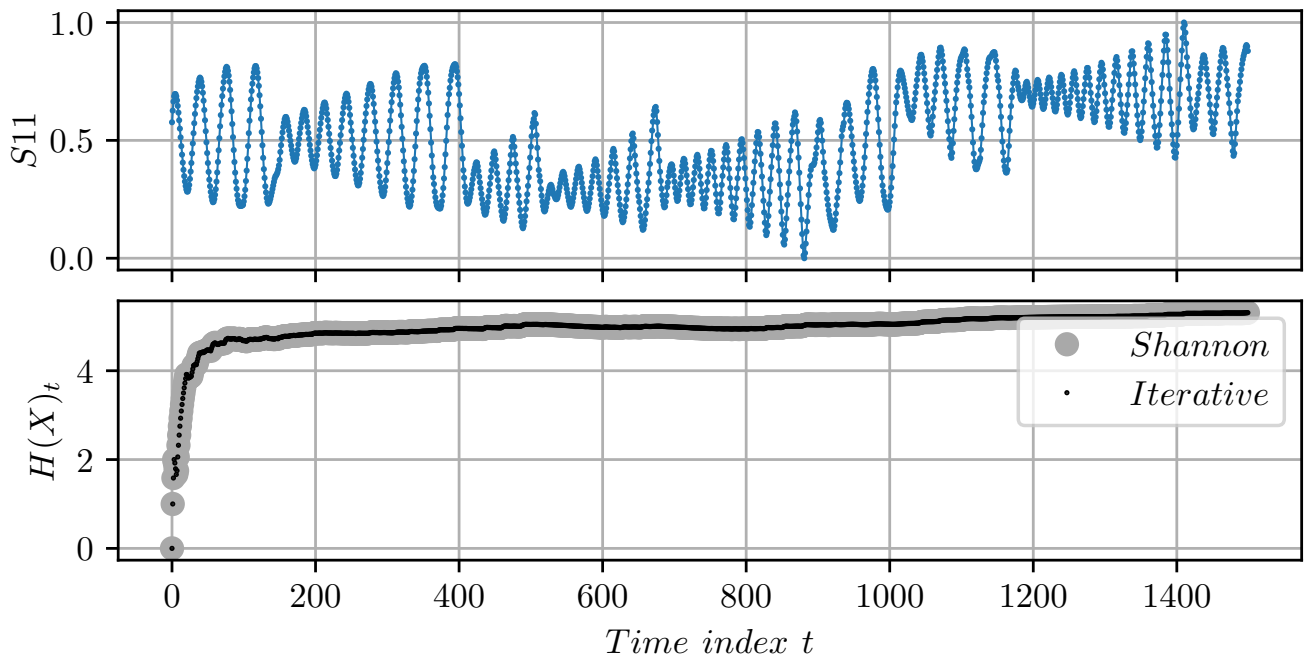

Figure B.22: $S 11$ and its instantaneous Entropy using Shannon's formula and its iterative form.

\section{B.3 Probability Shaping}

The histograms of $\check{r}_{X}$ and $\check{r}_{d X}$ are shown in Fig. B.23 to Fig. B.33. Notice how most of the histograms are skewed to the lower ranking side. Again this asserts the intuition discussed earlier where few samples carry most of the information in the signal. 
$S 1$

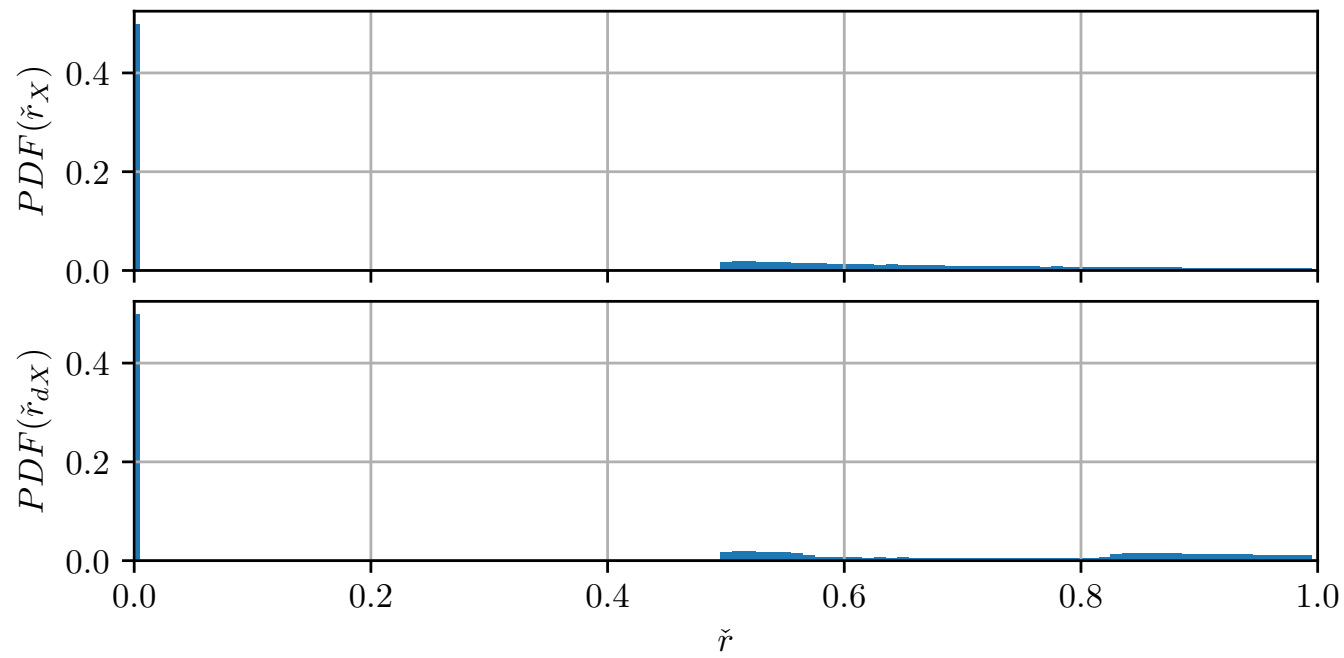

Figure B.23: PDF of $\check{r}_{X}$ and $\check{r}_{d X}$ for $S 1$.

$S 2$

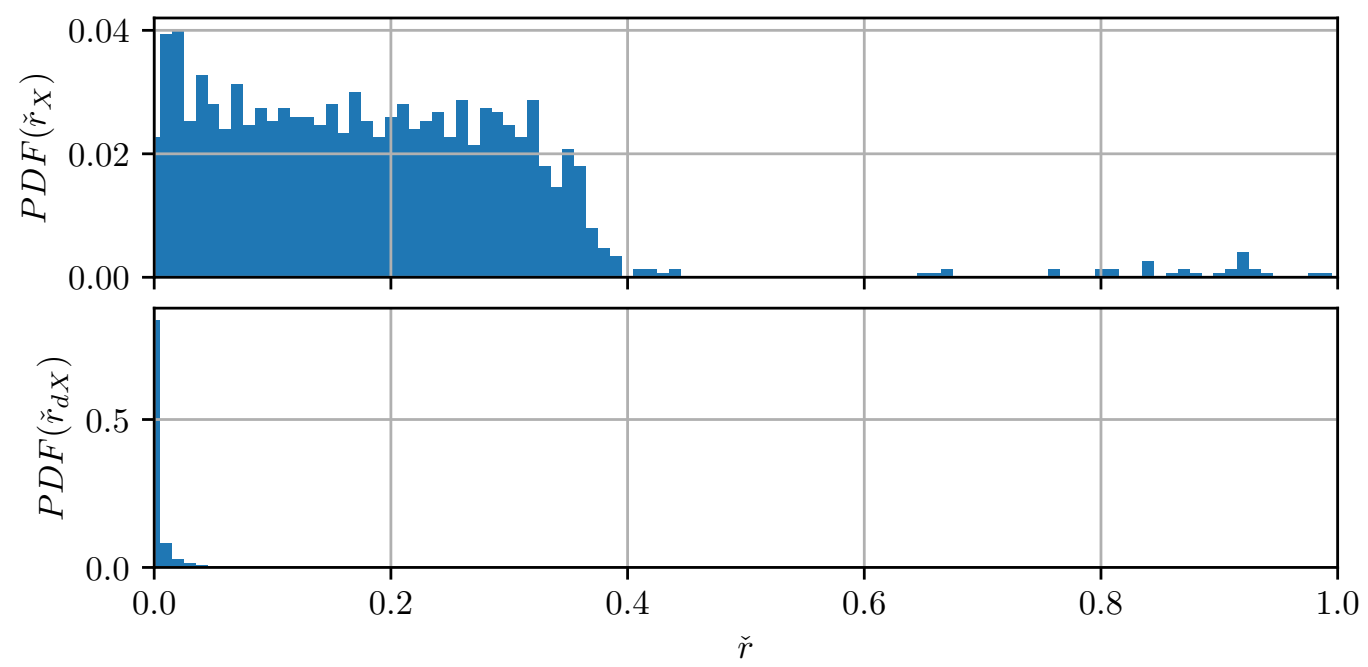

Figure B.24: PDF of $\check{r}_{X}$ and $\check{r}_{d X}$ for $S 2$. 
$S 3$
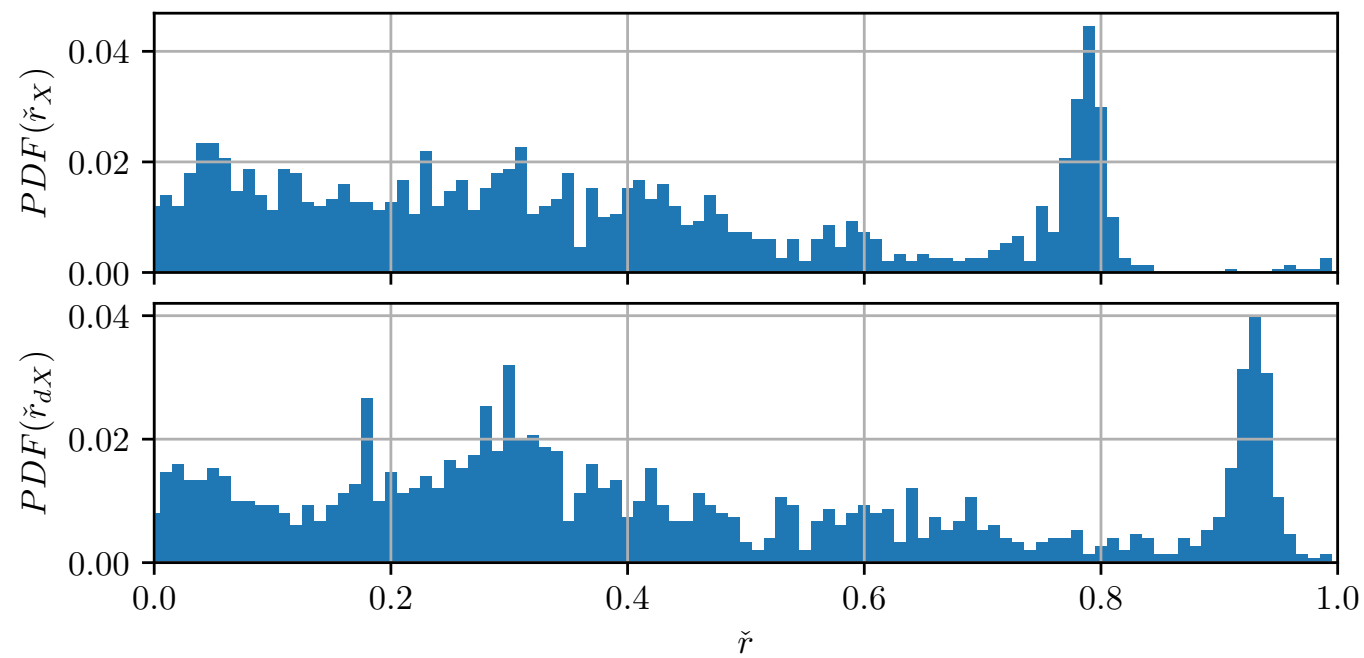

Figure B.25: PDF of $\check{r}_{X}$ and $\check{r}_{d X}$ for $S 3$.

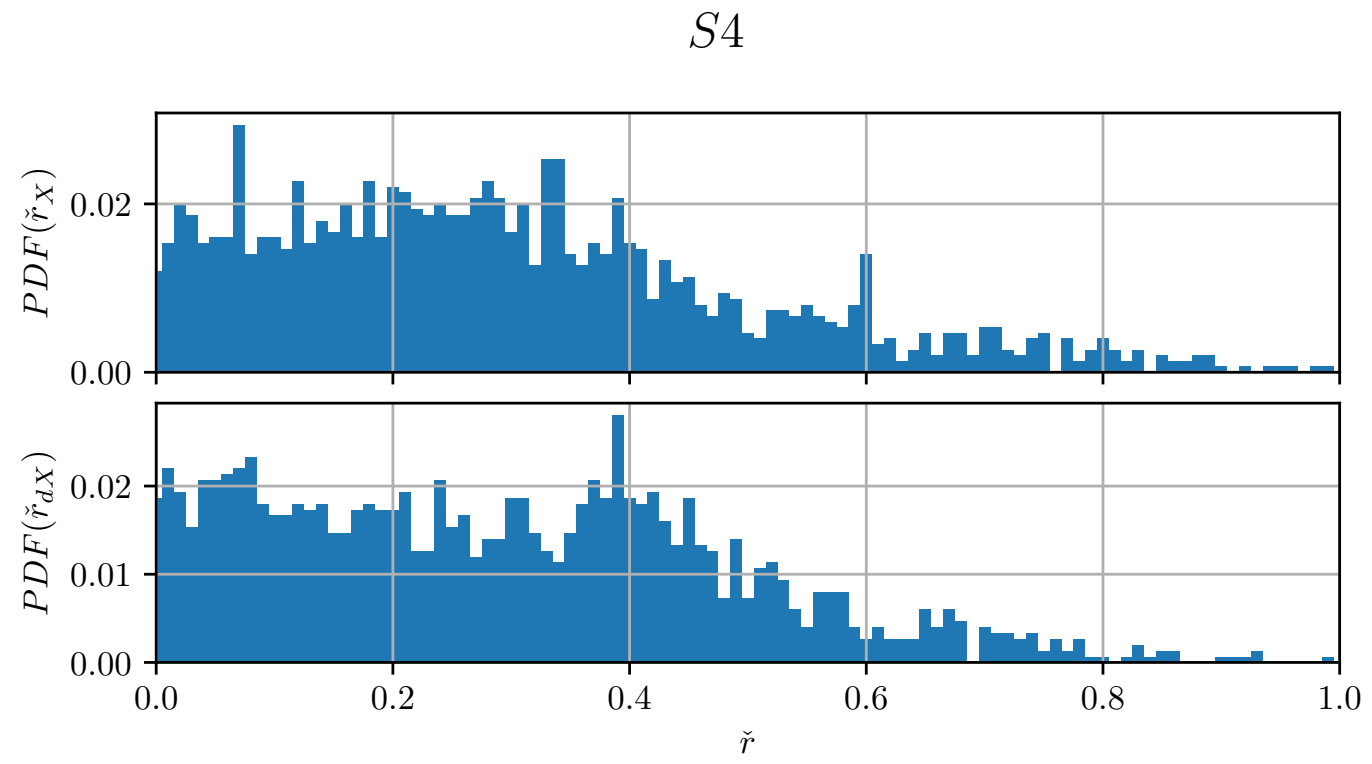

Figure B.26: PDF of $\check{r}_{X}$ and $\check{r}_{d X}$ for $S 4$. 


\section{S5}

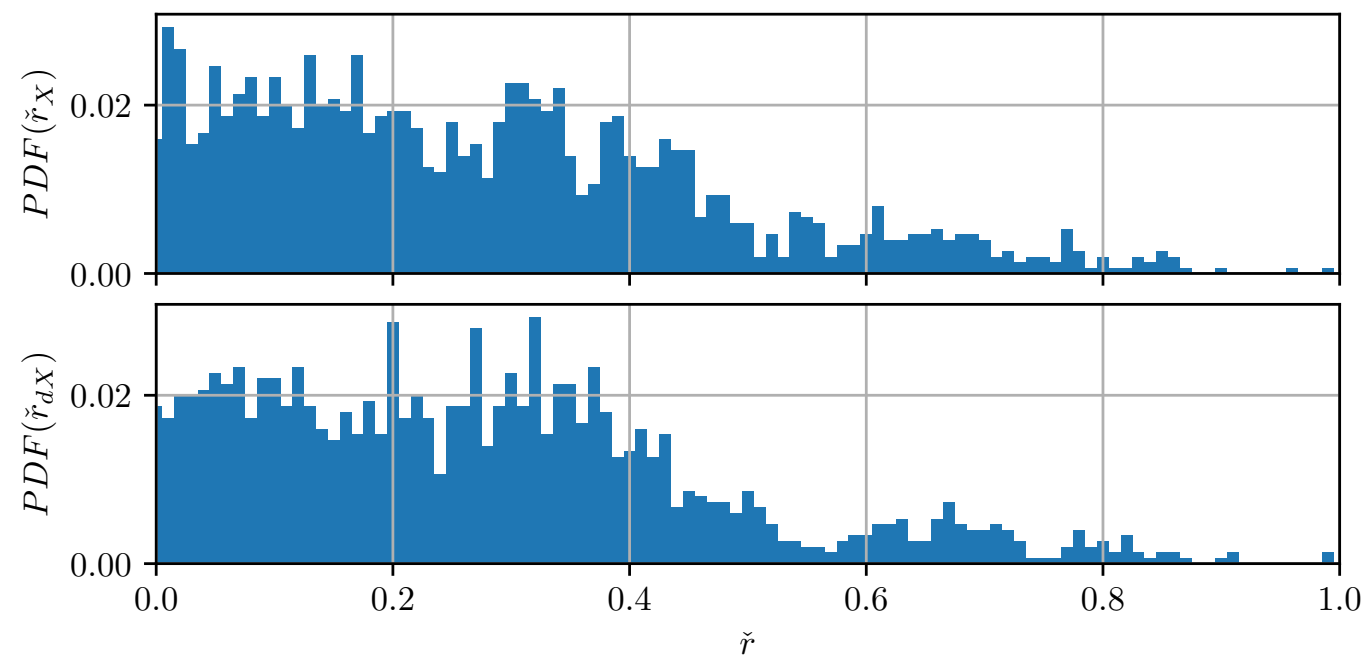

Figure B.27: PDF of $\check{r}_{X}$ and $\check{r}_{d X}$ for $S 5$.

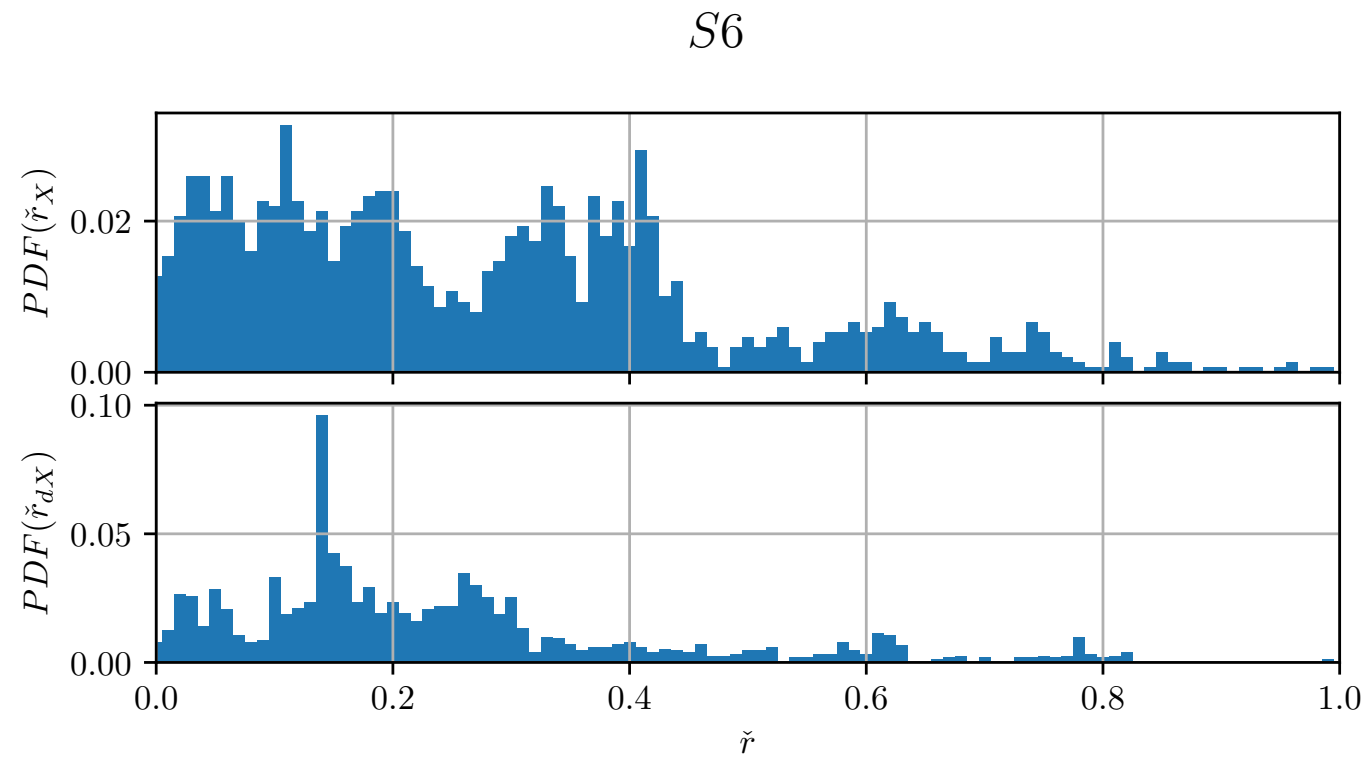

Figure B.28: PDF of $\check{r}_{X}$ and $\check{r}_{d X}$ for $S 6$. 


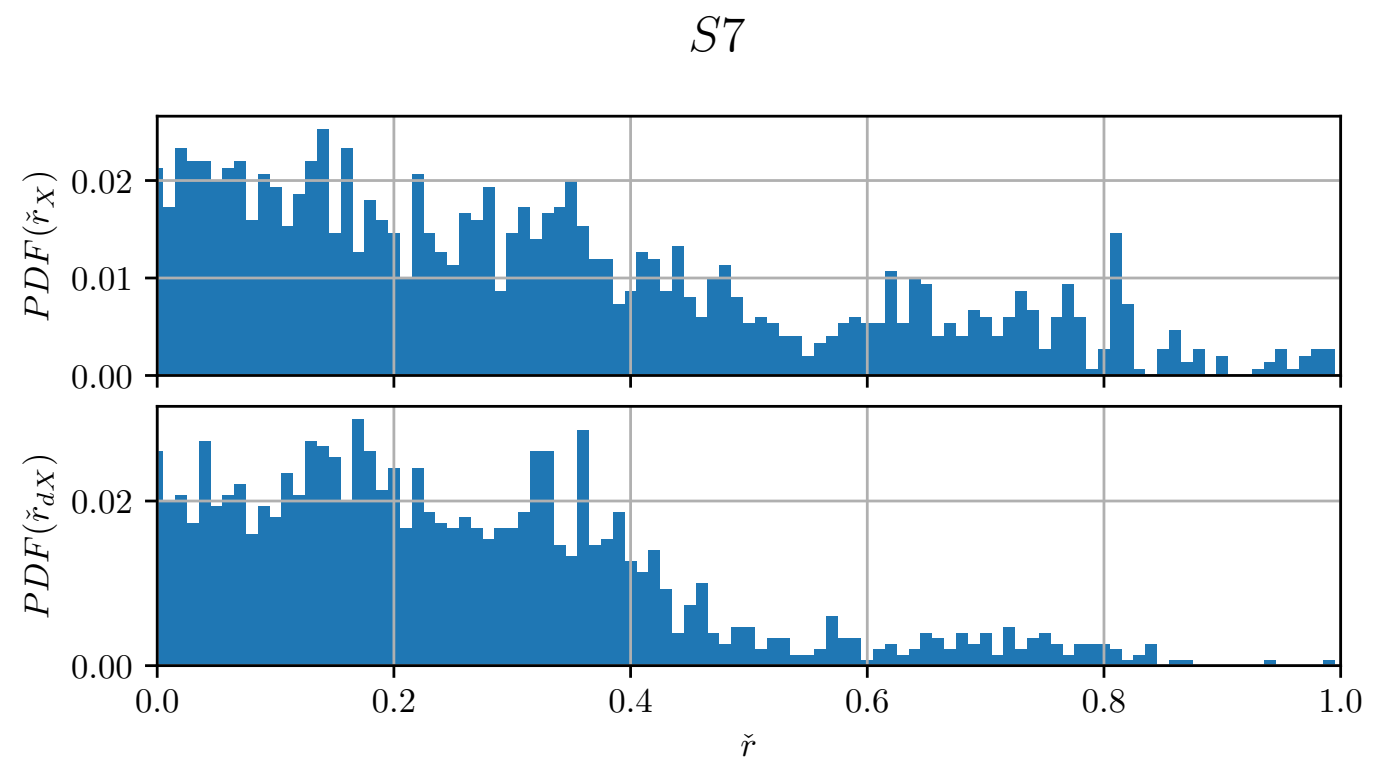

Figure B.29: PDF of $\check{r}_{X}$ and $\check{r}_{d X}$ for $S 7$.

$S 8$

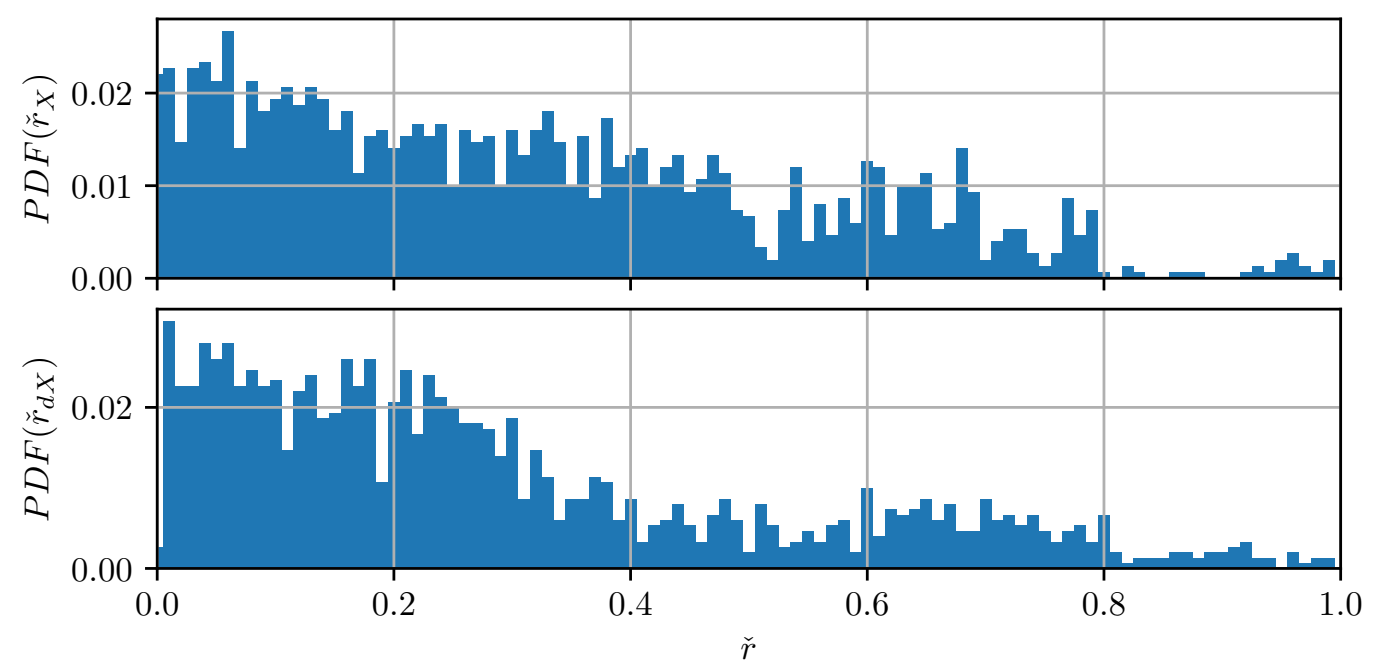

Figure B.30: PDF of $\check{r}_{X}$ and $\check{r}_{d X}$ for $S 8$. 


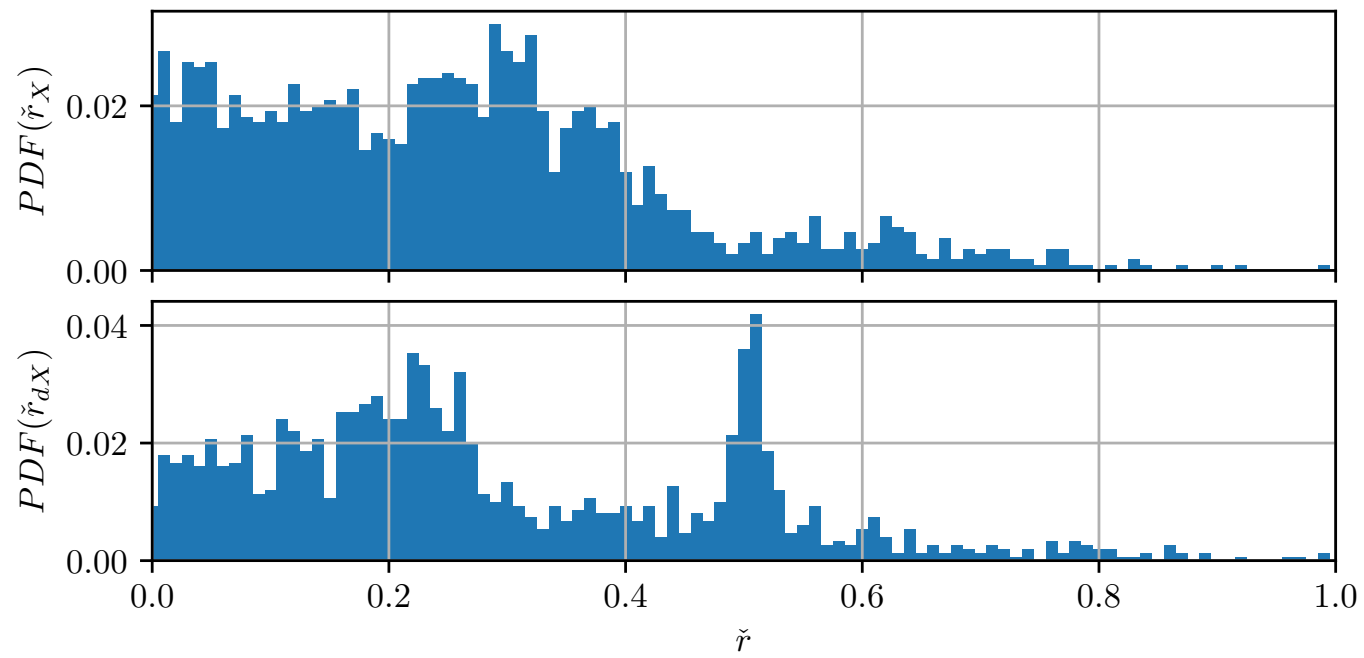

Figure B.31: PDF of $\check{r}_{X}$ and $\check{r}_{d X}$ for $S 9$.

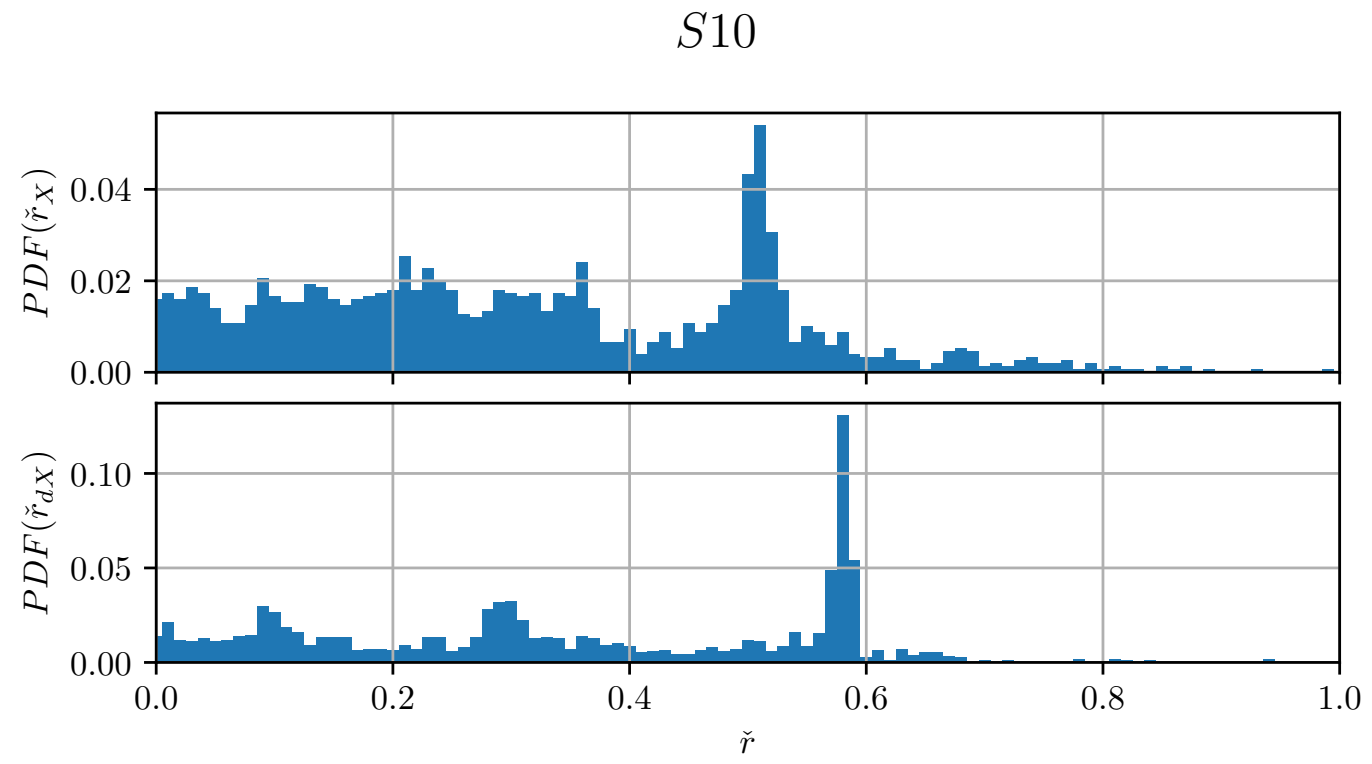

Figure B.32: PDF of $\check{r}_{X}$ and $\check{r}_{d X}$ for $S 10$. 


\section{$S 11$}

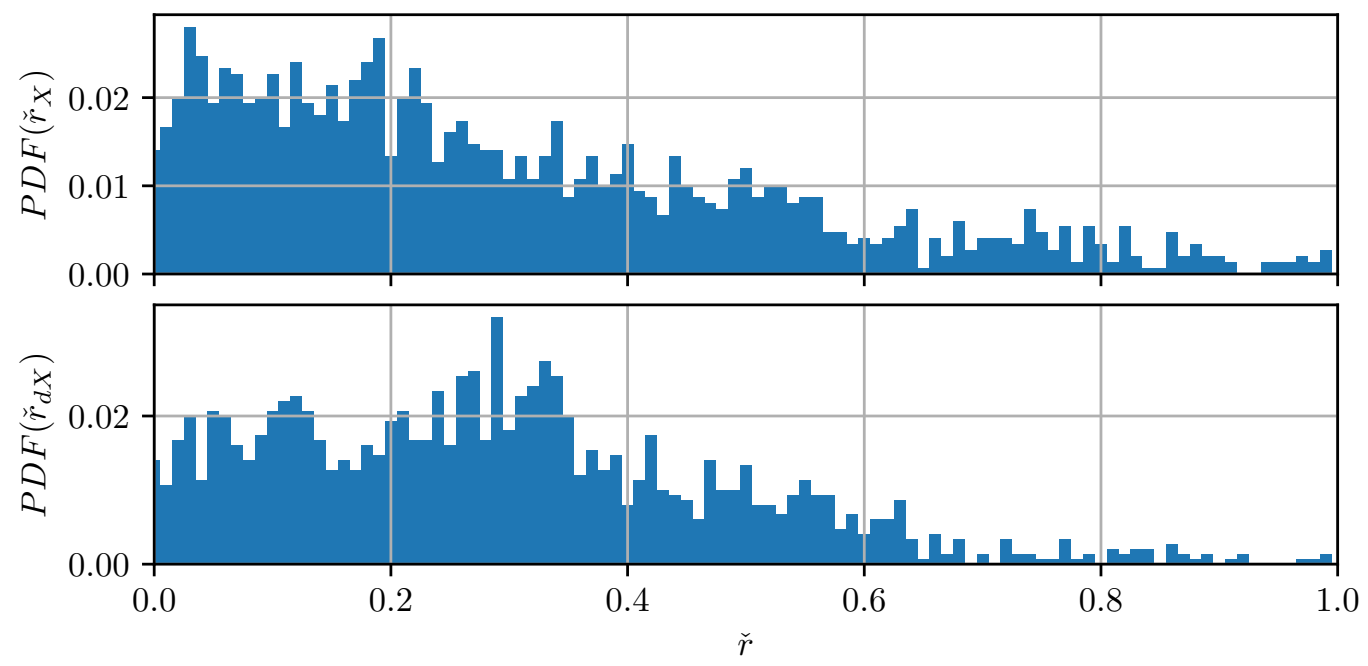

Figure B.33: PDF of $\check{r}_{X}$ and $\check{r}_{d X}$ for $S 11$.

\section{B.4 Evaluation}

In Fig. B.34 to Fig. B.44, a comparison in the Entropic Sensing (ES) performance among different PSFs is shown. Note that with almost all signals, the "High Entropy" PSF has the least $S R R$. 


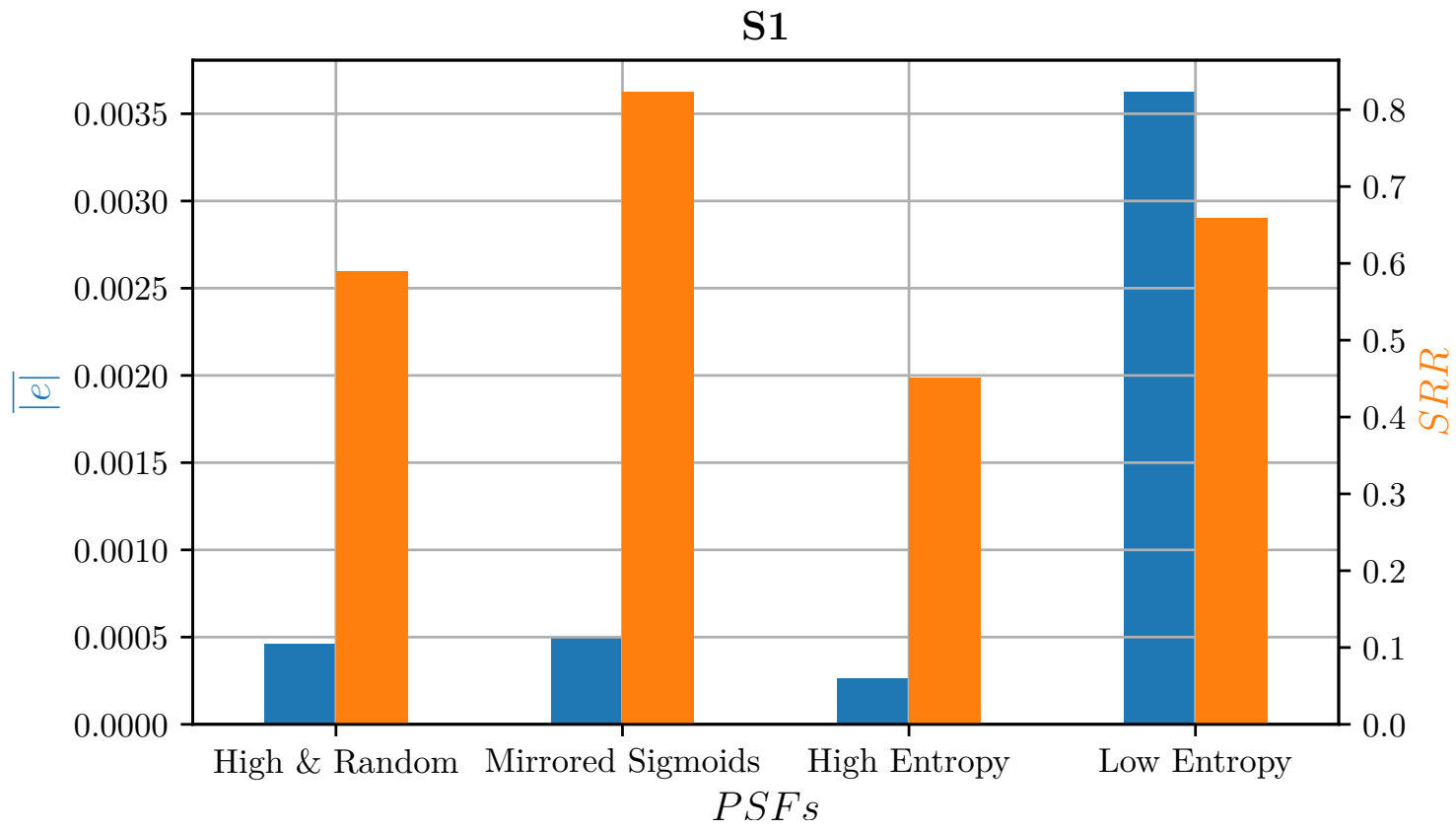

Figure B.34: $\overline{|e|}$ and $S R R$ among all the PSFs for $S 1$.

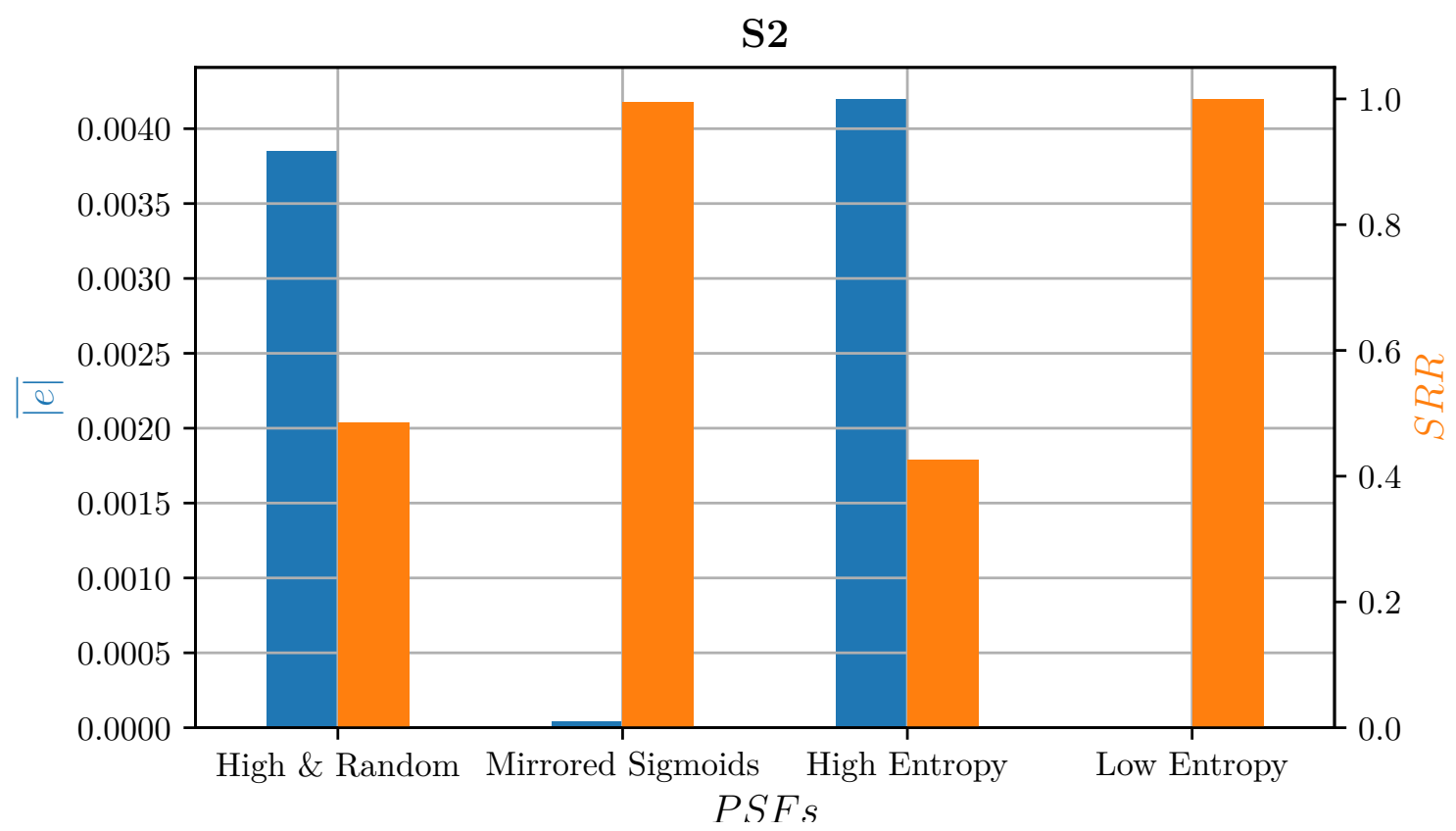

Figure B.35: $\overline{|e|}$ and $S R R$ among all the PSFs for $S 2$. 


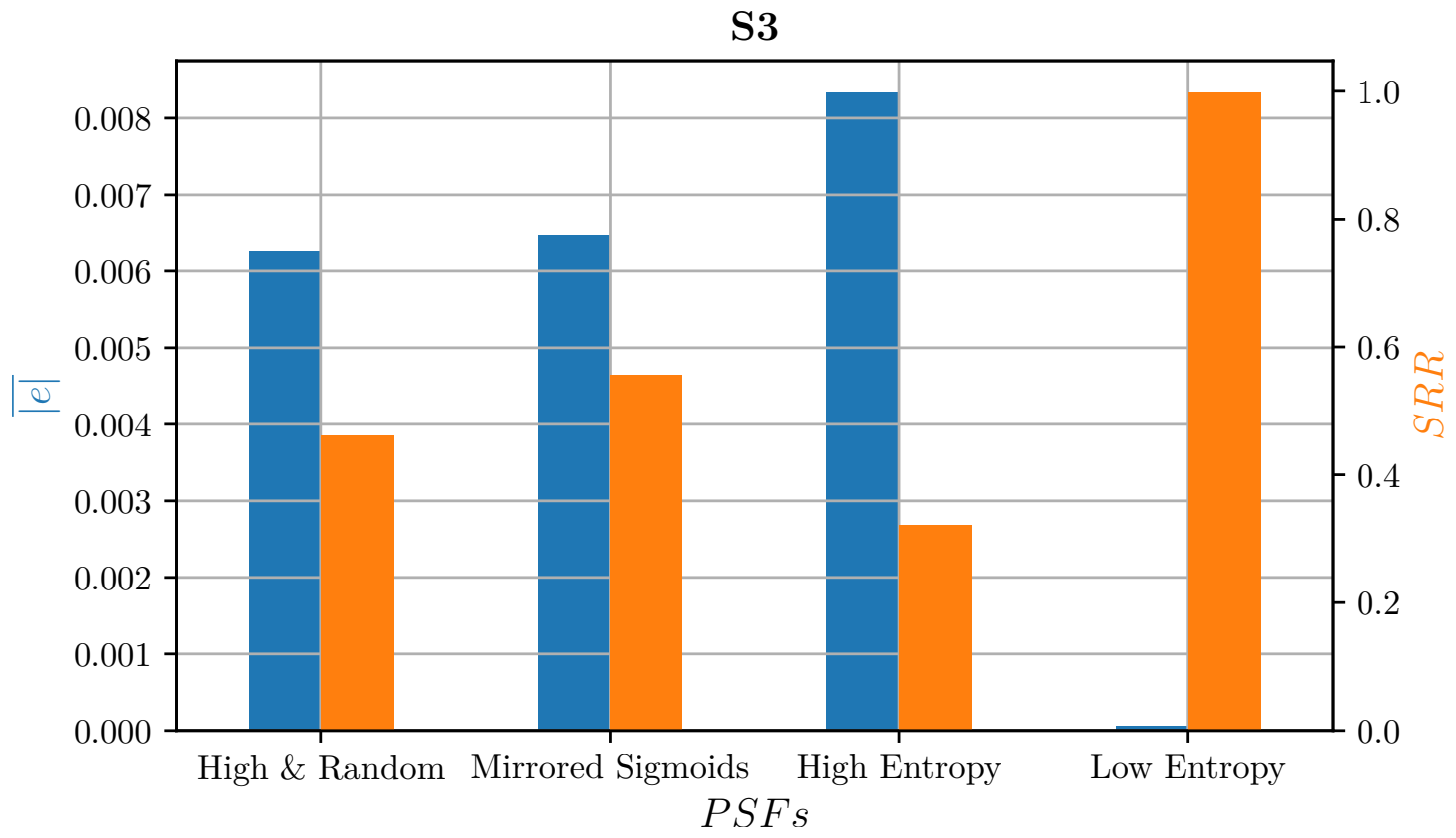

Figure B.36: $\overline{|e|}$ and $S R R$ among all the PSFs for $S 3$.

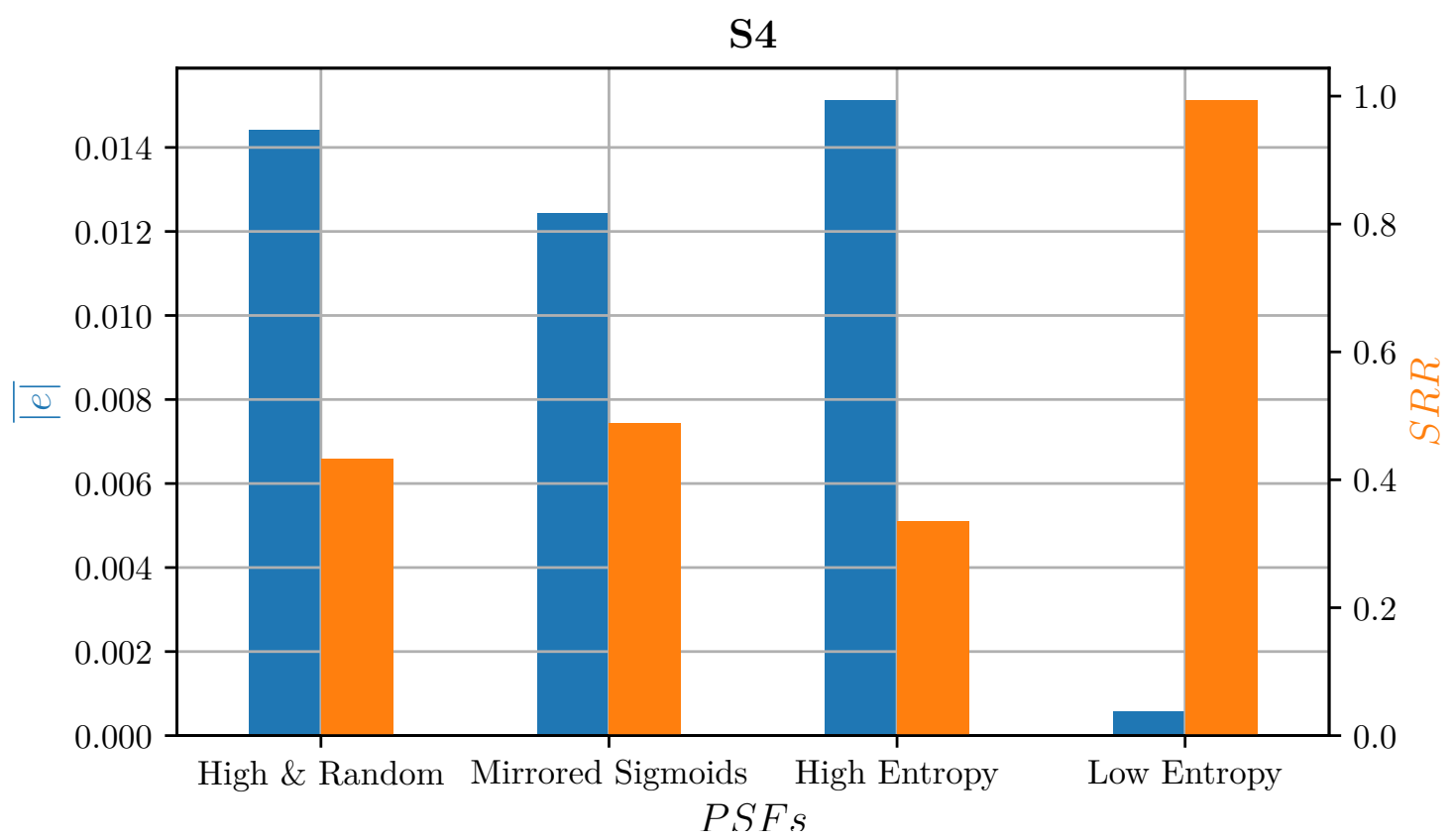

Figure B.37: $\overline{|e|}$ and $S R R$ among all the PSFs for $S 4$. 
S5

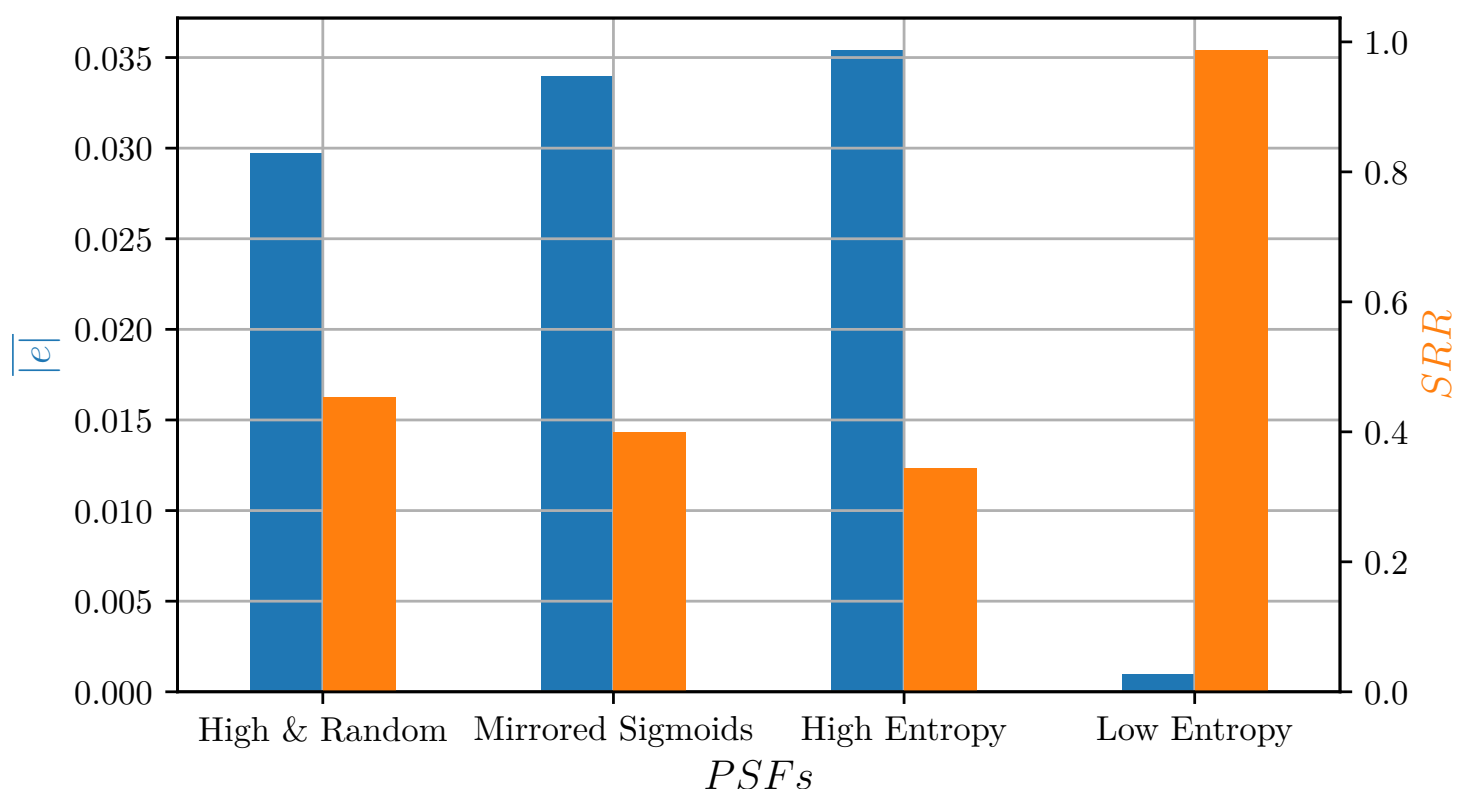

Figure B.38: $\overline{|e|}$ and $S R R$ among all the PSFs for $S 5$.

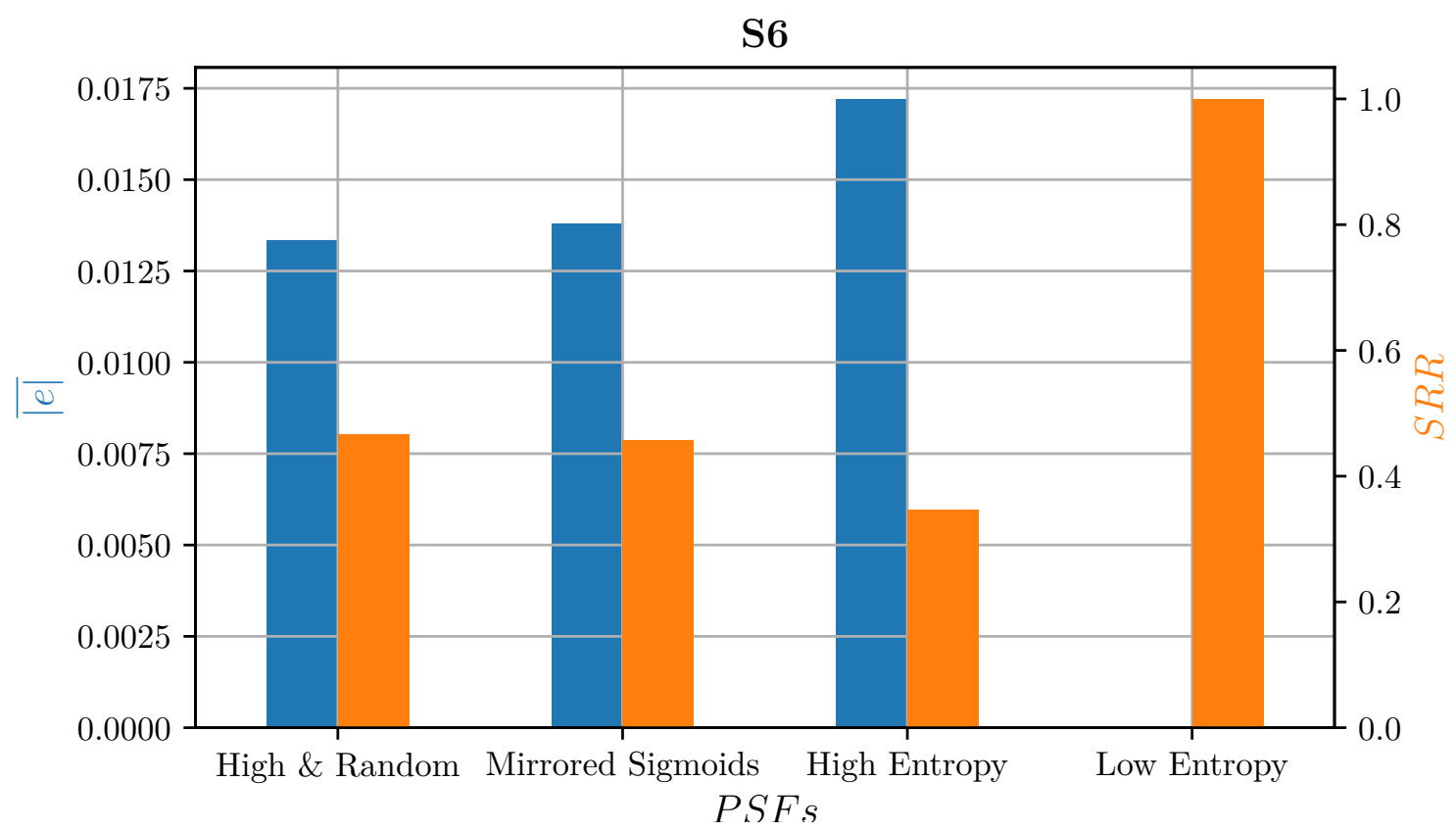

Figure B.39: $\overline{|e|}$ and $S R R$ among all the PSFs for $S 6$. 


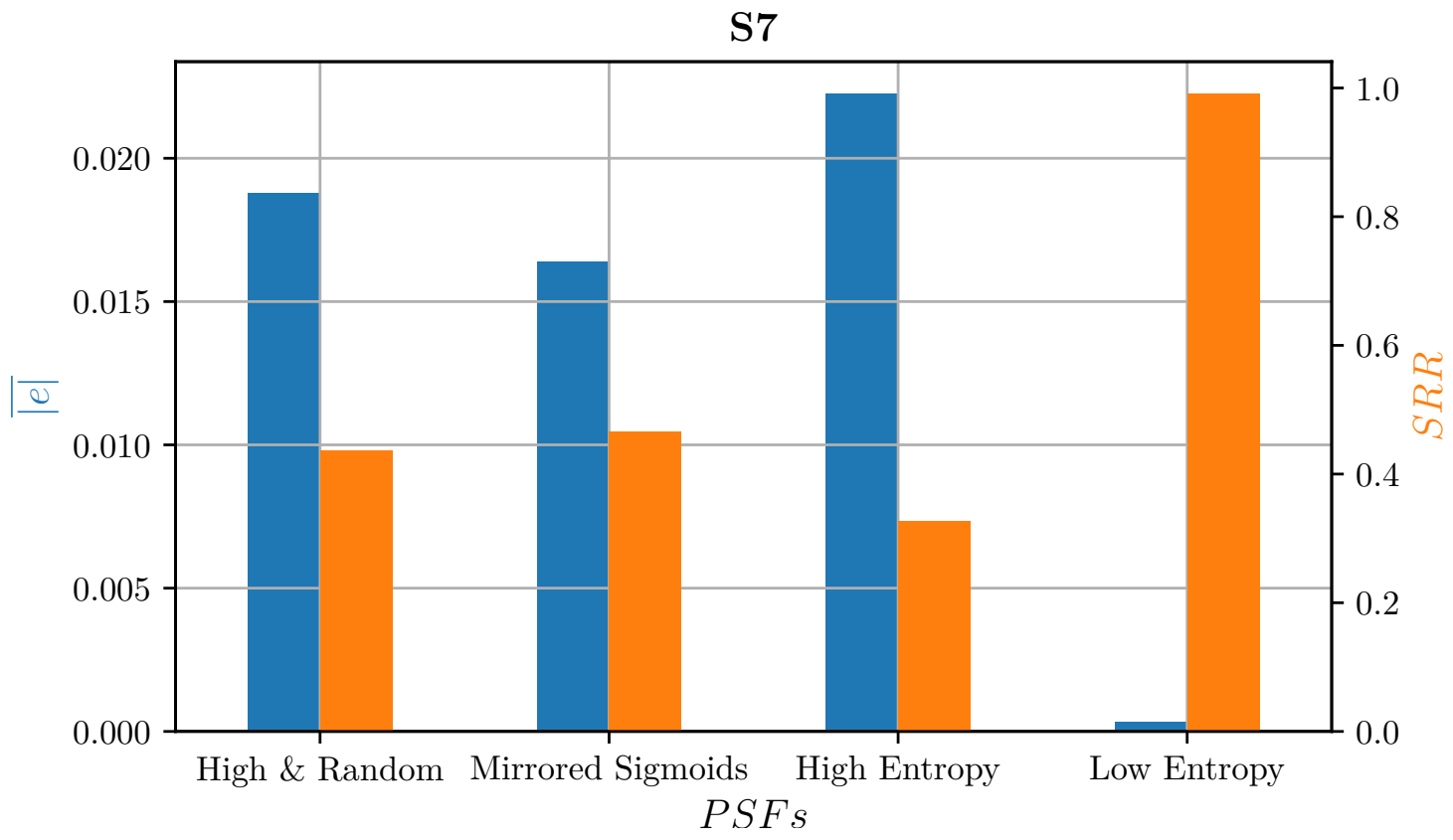

Figure B.40: $\overline{|e|}$ and $S R R$ among all the PSFs for $S 7$.

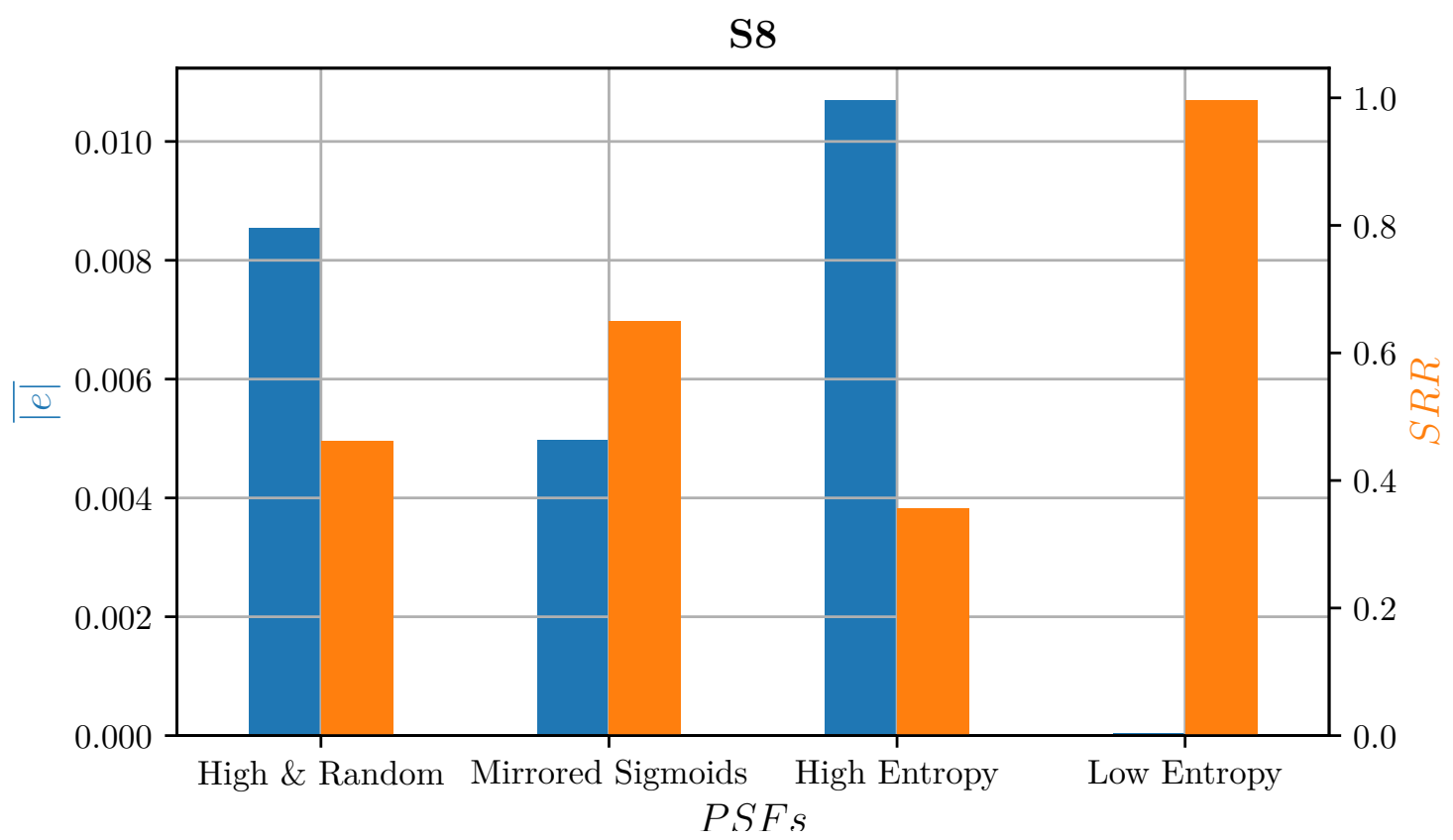

Figure B.41: $\overline{|e|}$ and $S R R$ among all the PSFs for $S 8$. 


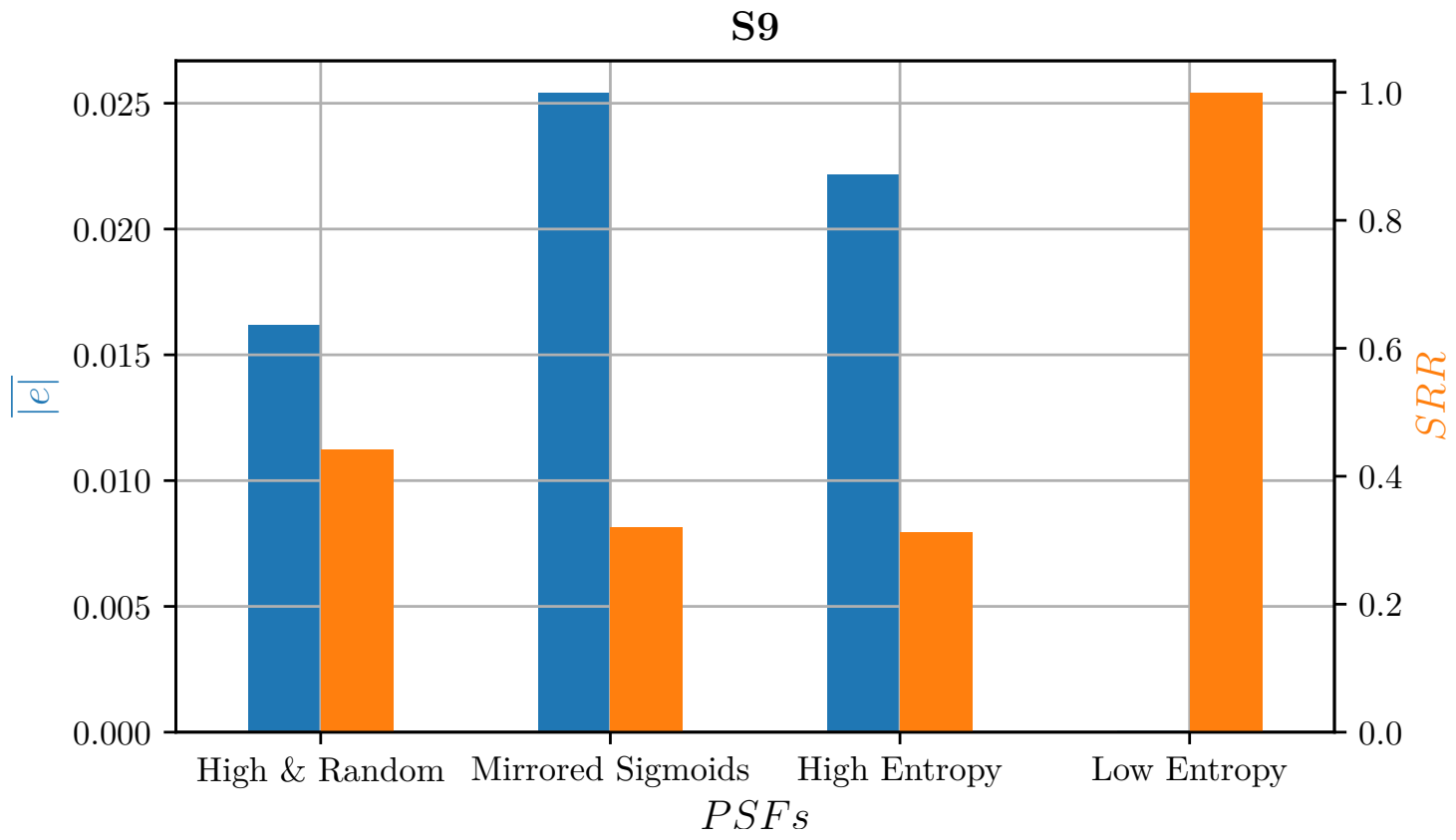

Figure B.42: $\overline{|e|}$ and $S R R$ among all the PSFs for $S 9$.

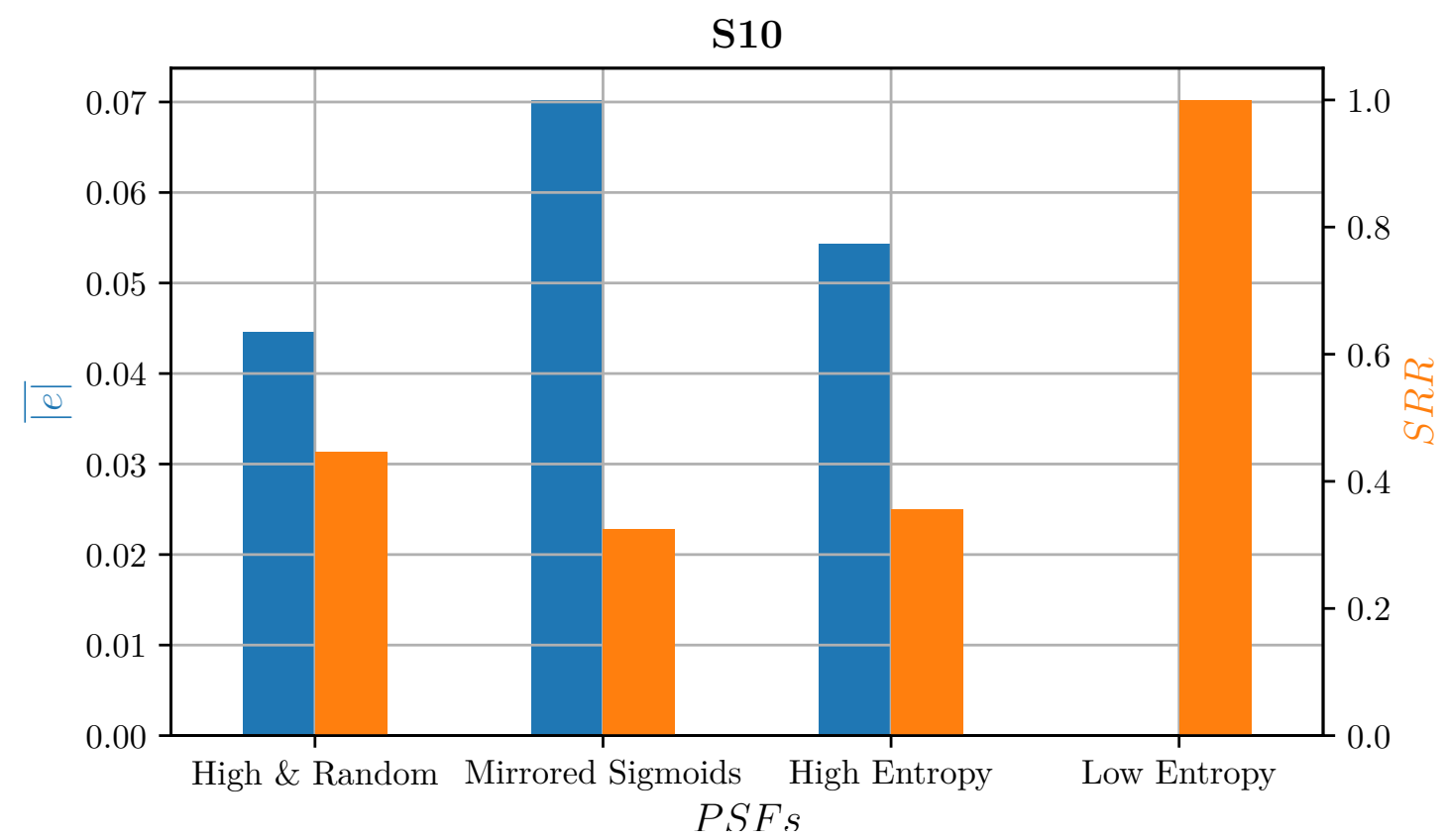

Figure B.43: $\overline{|e|}$ and $S R R$ among all the PSFs for $S 10$. 


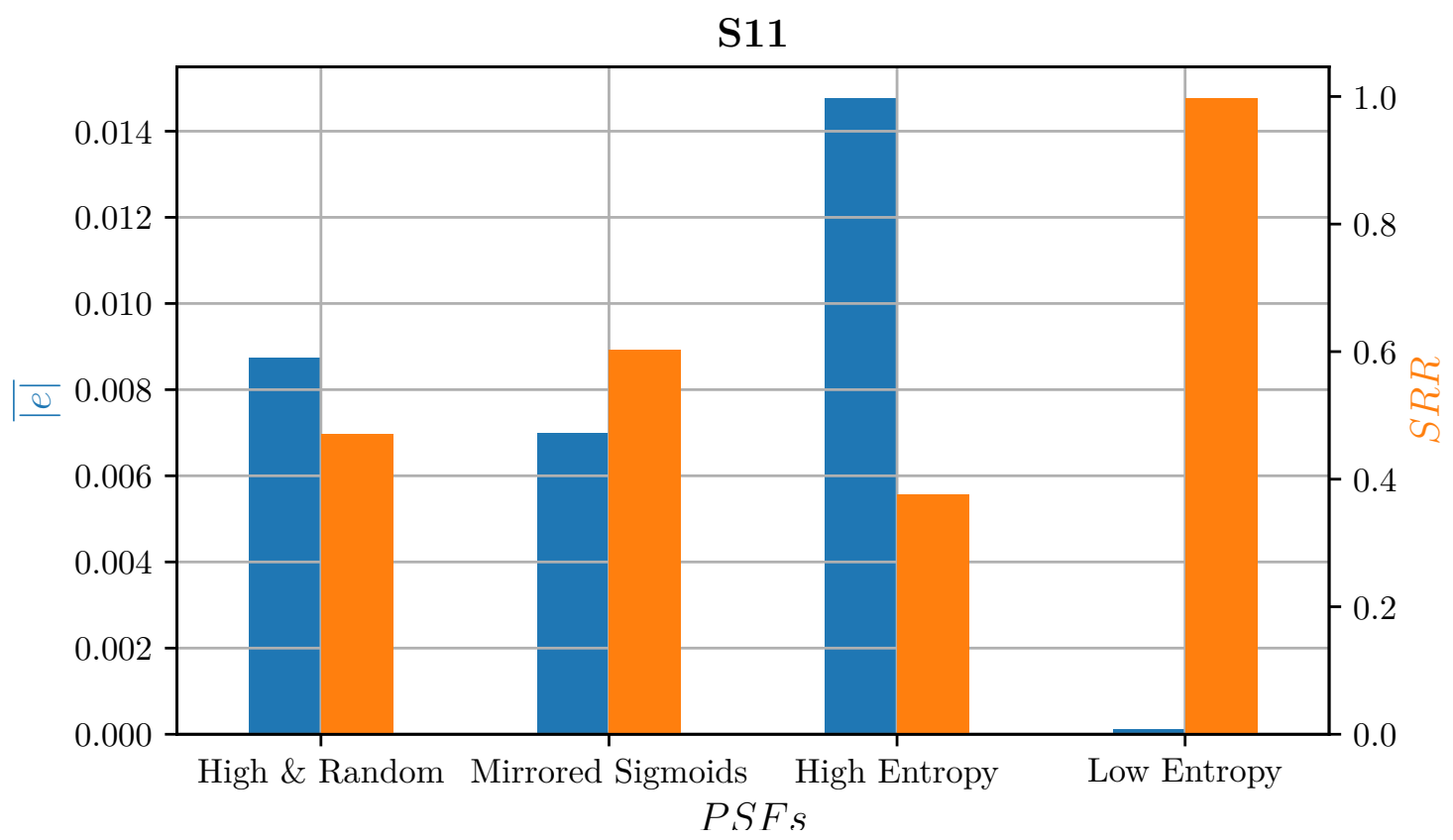

Figure B.44: $\overline{|e|}$ and $S R R$ among all the PSFs for $S 11$.

The second group of figures in this section show the instantaneous error in the recovered signal. Note that the error diminishes with chaotic, and periodic signals with low noise as shown in Fig. B.47, Fig. B.48, and Fig. B.53 to Fig. 5.10. Conversely, the error is non-diminishing in signals with noise and random signals as shown in Fig. B.45, Fig. B.46, and Fig. B.49 to Fig. B.52. 
S1 with PSF=High Entropy

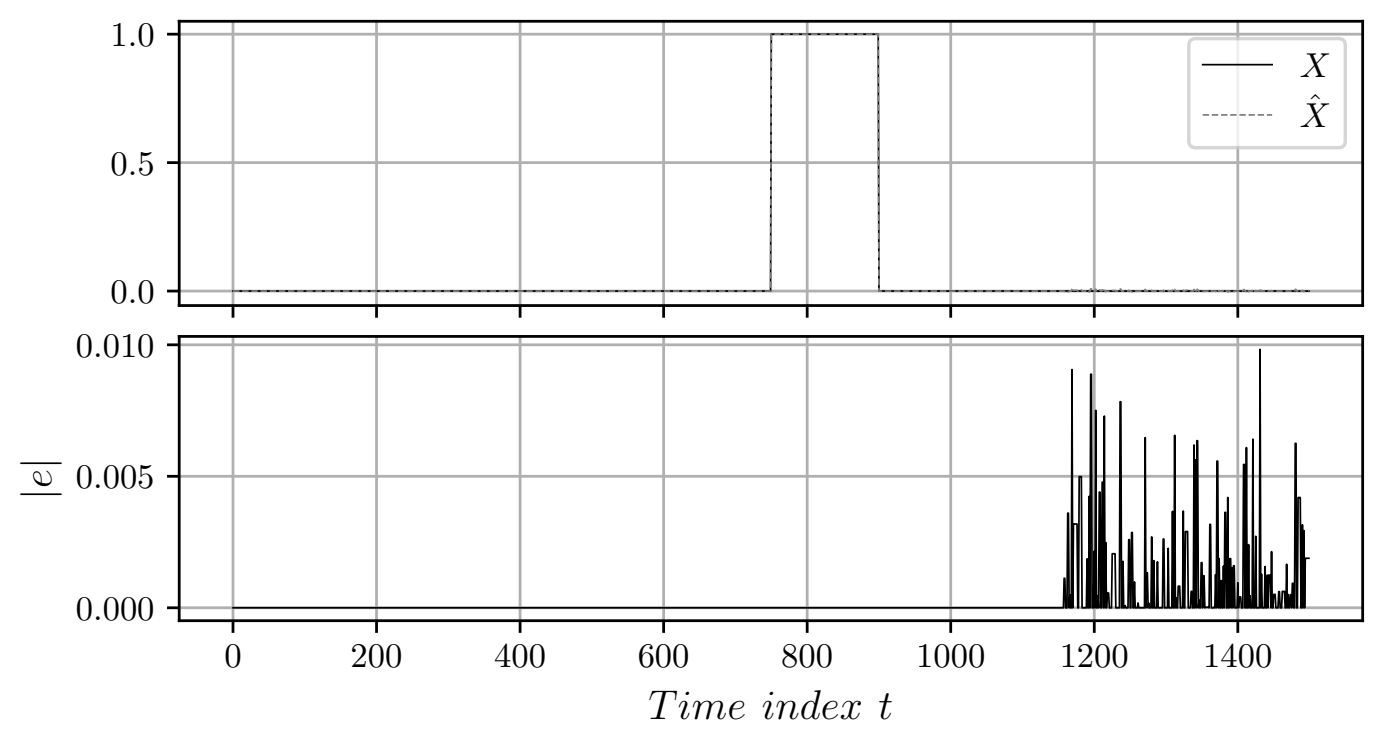

Figure B.45: Top: $X$ and $\hat{X}$. Bottom: Instantaneous absolute error $|e|$.

S2 with PSF=High Entropy

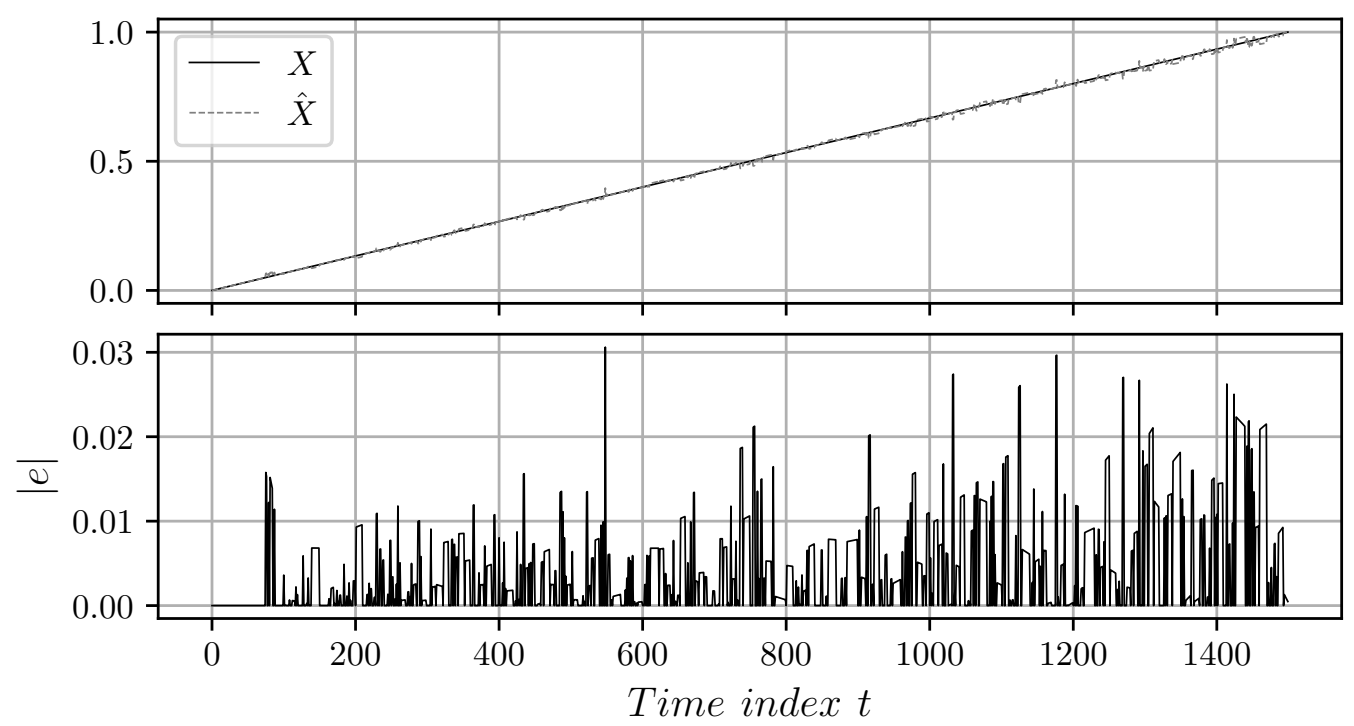

Figure B.46: Top: $X$ and $\hat{X}$. Bottom: Instantaneous absolute error $|e|$. 
S3 with PSF=High Entropy

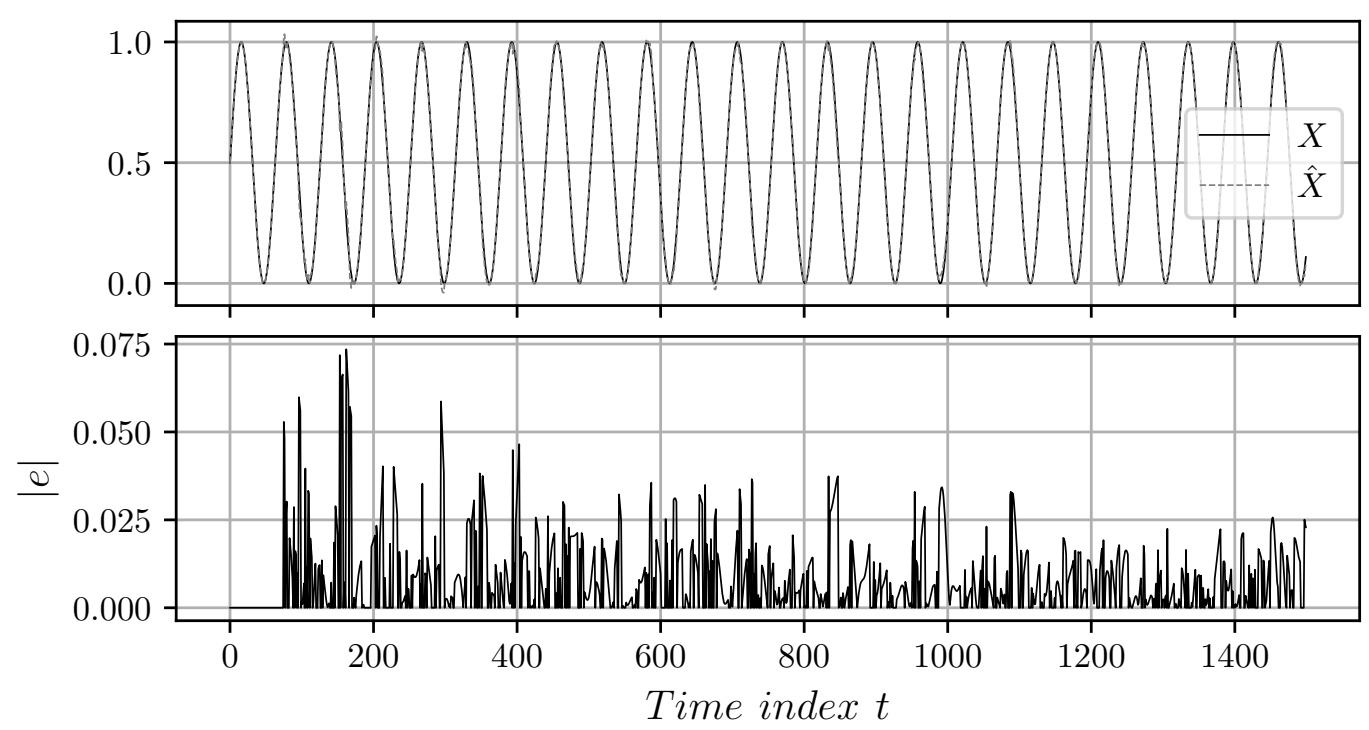

Figure B.47: Top: $X$ and $\hat{X}$. Bottom: Instantaneous absolute error $|e|$.

S4 with PSF=High Entropy
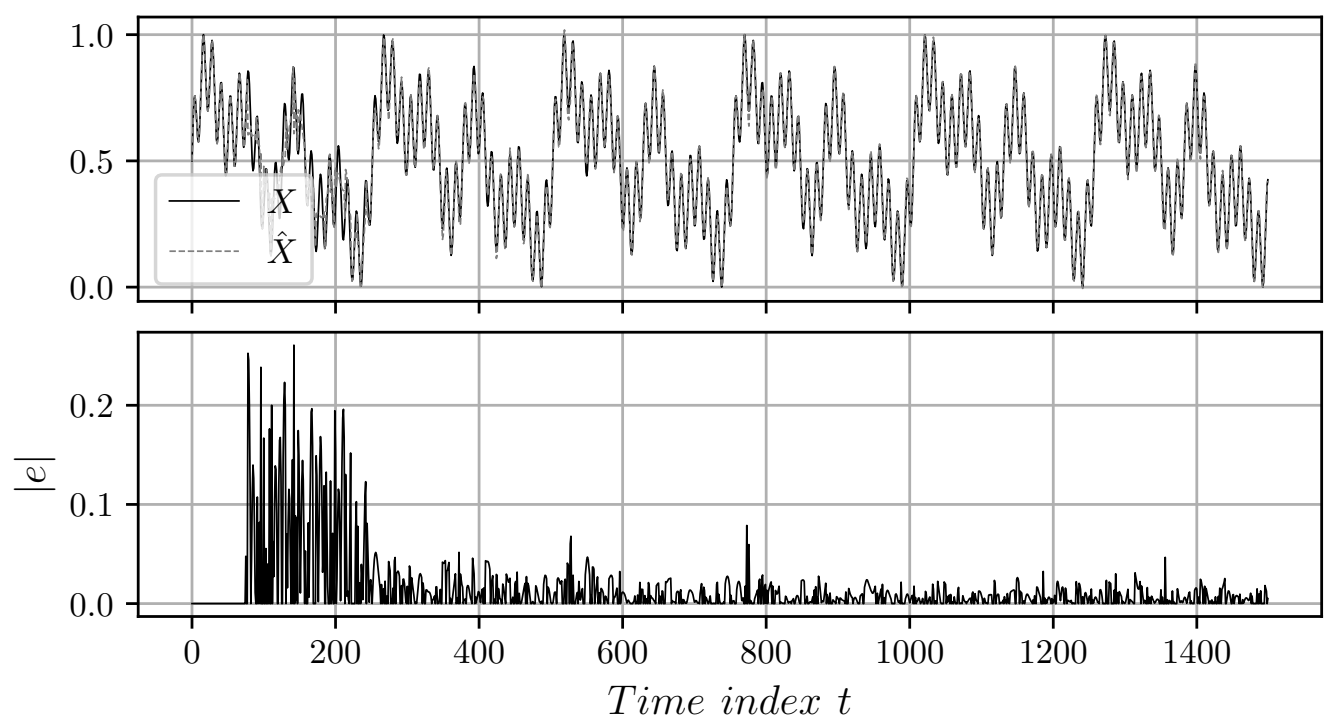

Figure B.48: Top: $X$ and $\hat{X}$. Bottom: Instantaneous absolute error $|e|$. 
S5 with PSF=High Entropy

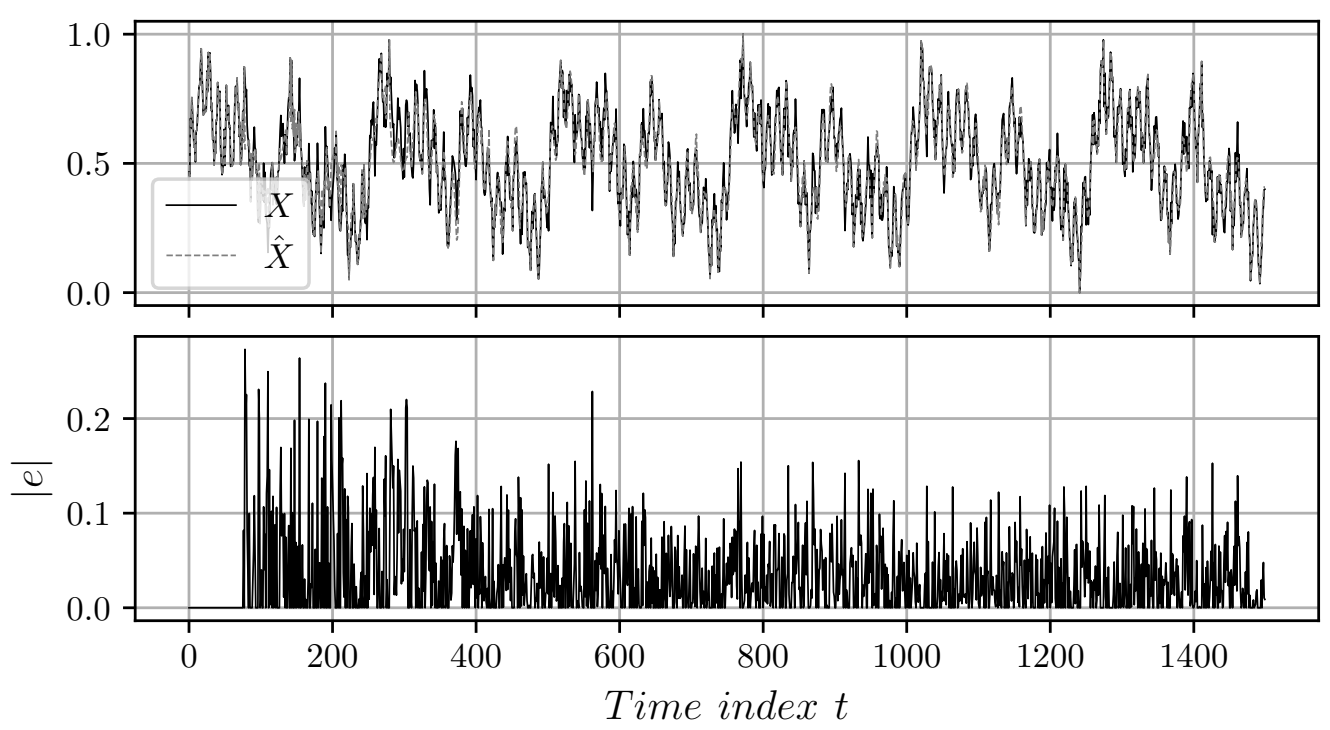

Figure B.49: Top: $X$ and $\hat{X}$. Bottom: Instantaneous absolute error $|e|$.

S6 with PSF=High Entropy

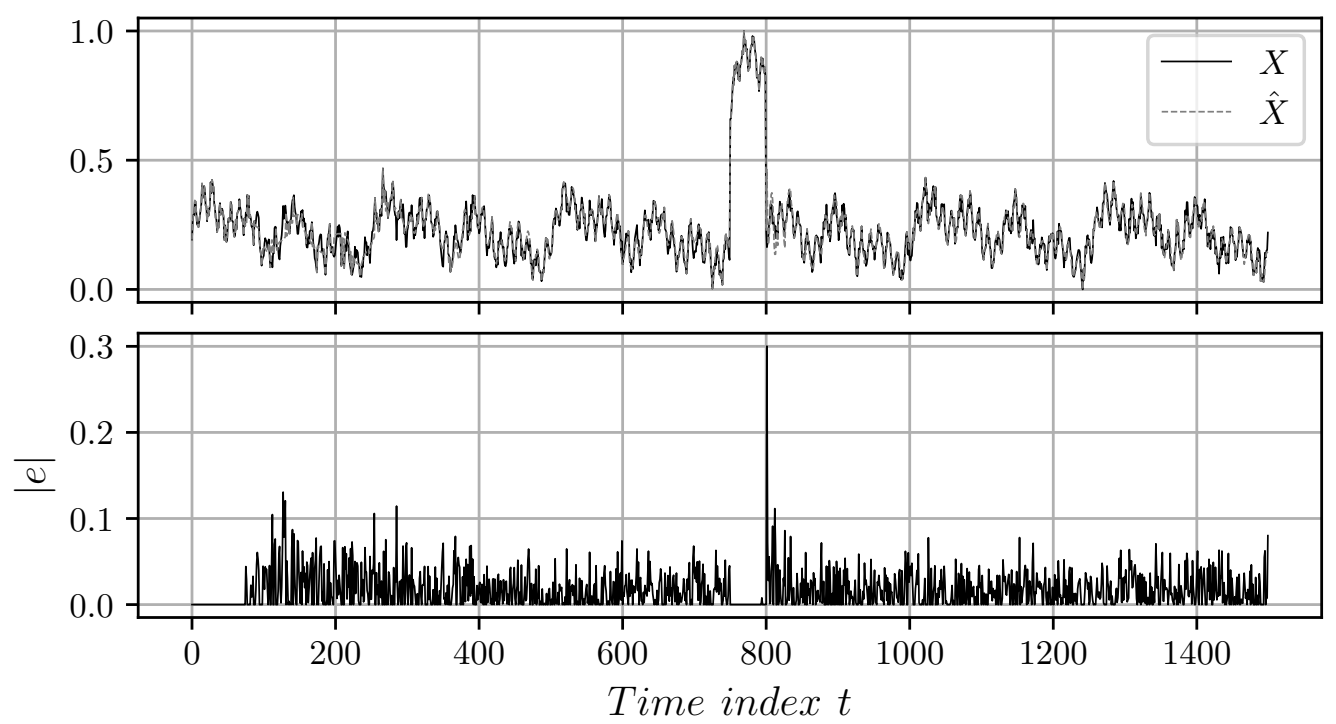

Figure B.50: Top: $X$ and $\hat{X}$. Bottom: Instantaneous absolute error $|e|$. 
S7 with PSF=High Entropy

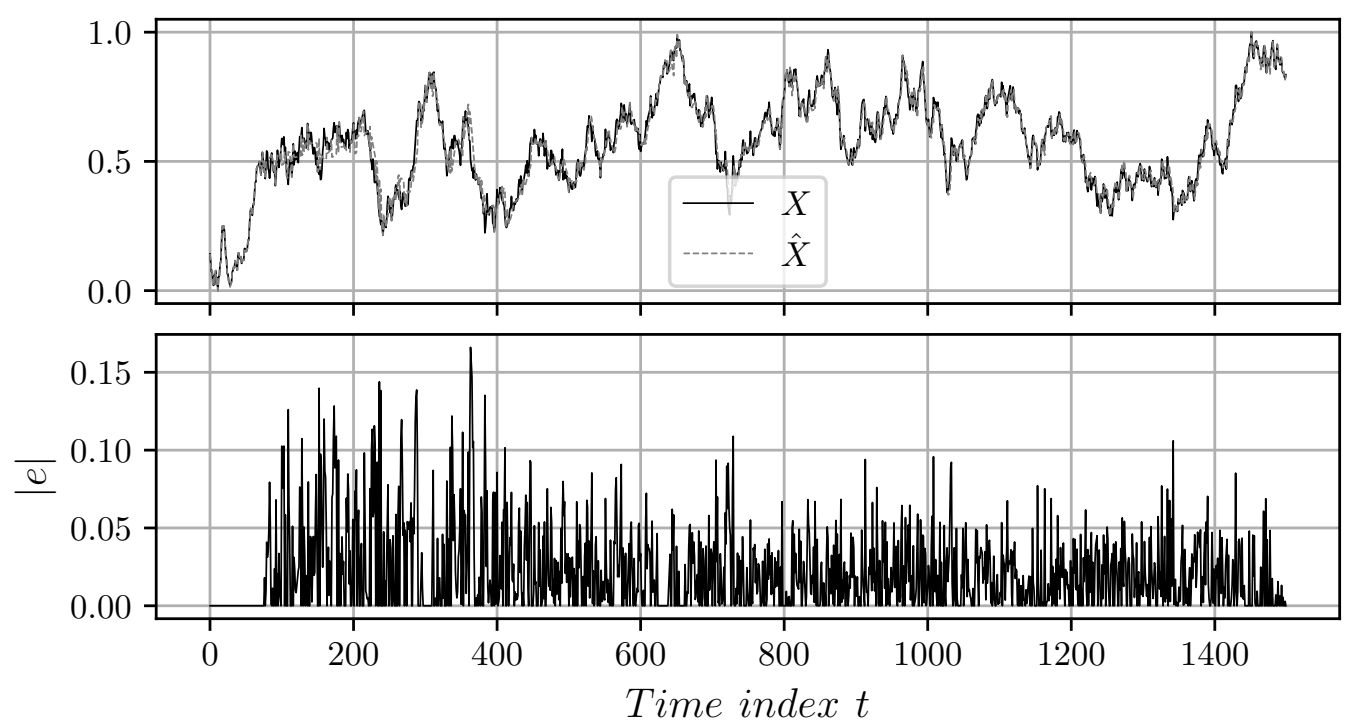

Figure B.51: Top: $X$ and $\hat{X}$. Bottom: Instantaneous absolute error $|e|$.

S8 with PSF = High Entropy

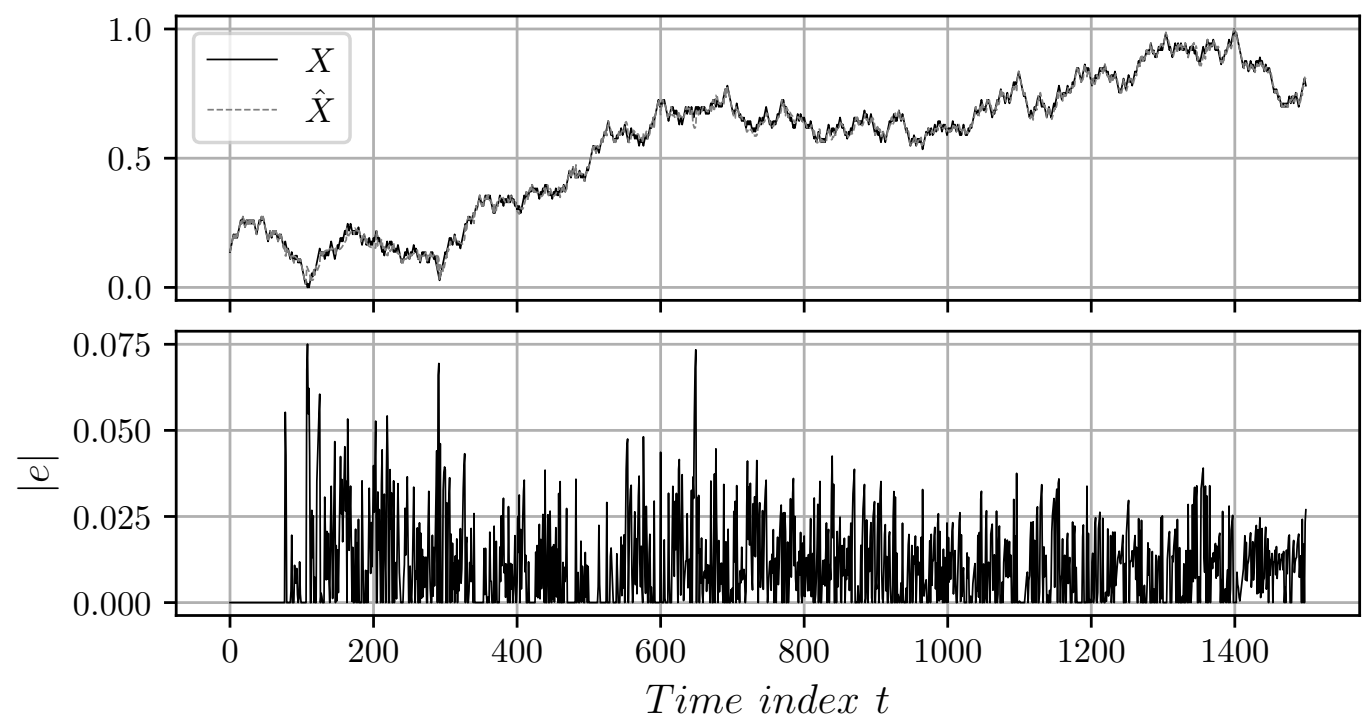

Figure B.52: Top: $X$ and $\hat{X}$. Bottom: Instantaneous absolute error $|e|$. 
S9 with PSF = High Entropy

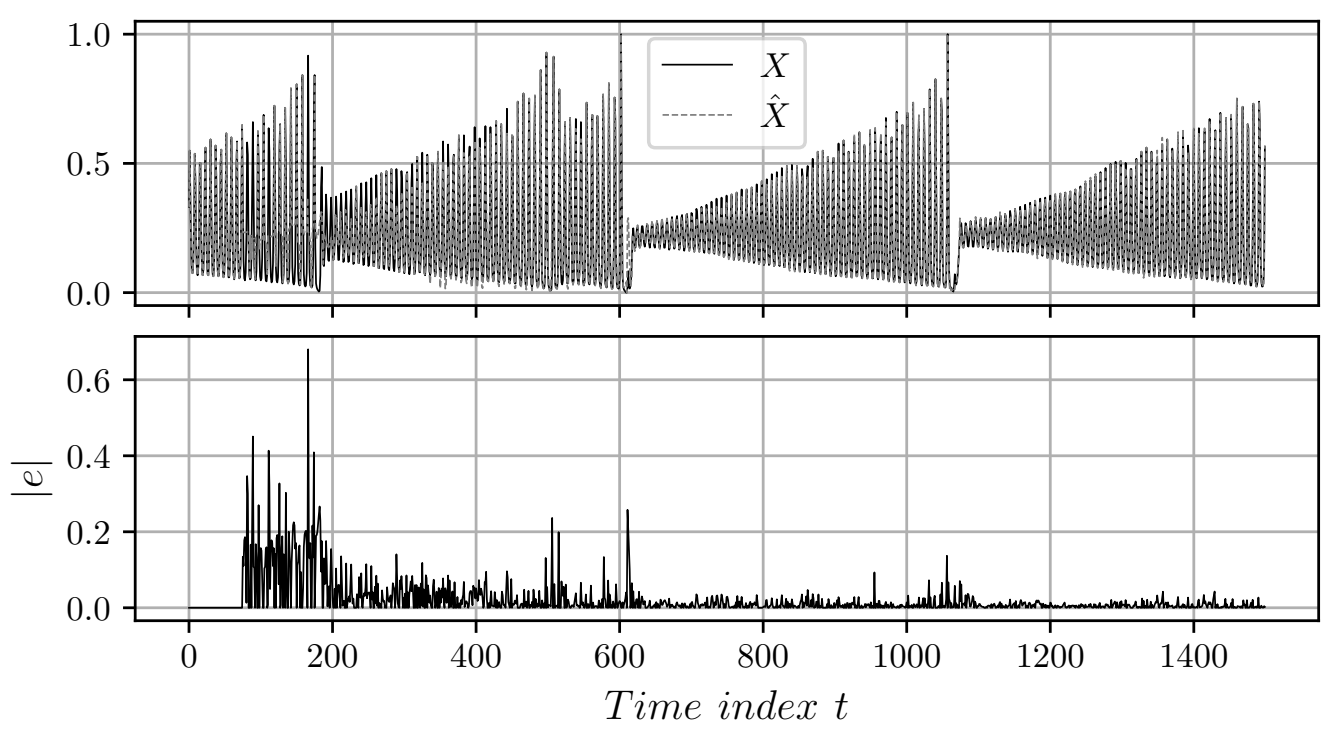

Figure B.53: Top: $X$ and $\hat{X}$. Bottom: Instantaneous absolute error $|e|$.

\section{S10 with PSF=High Entropy}

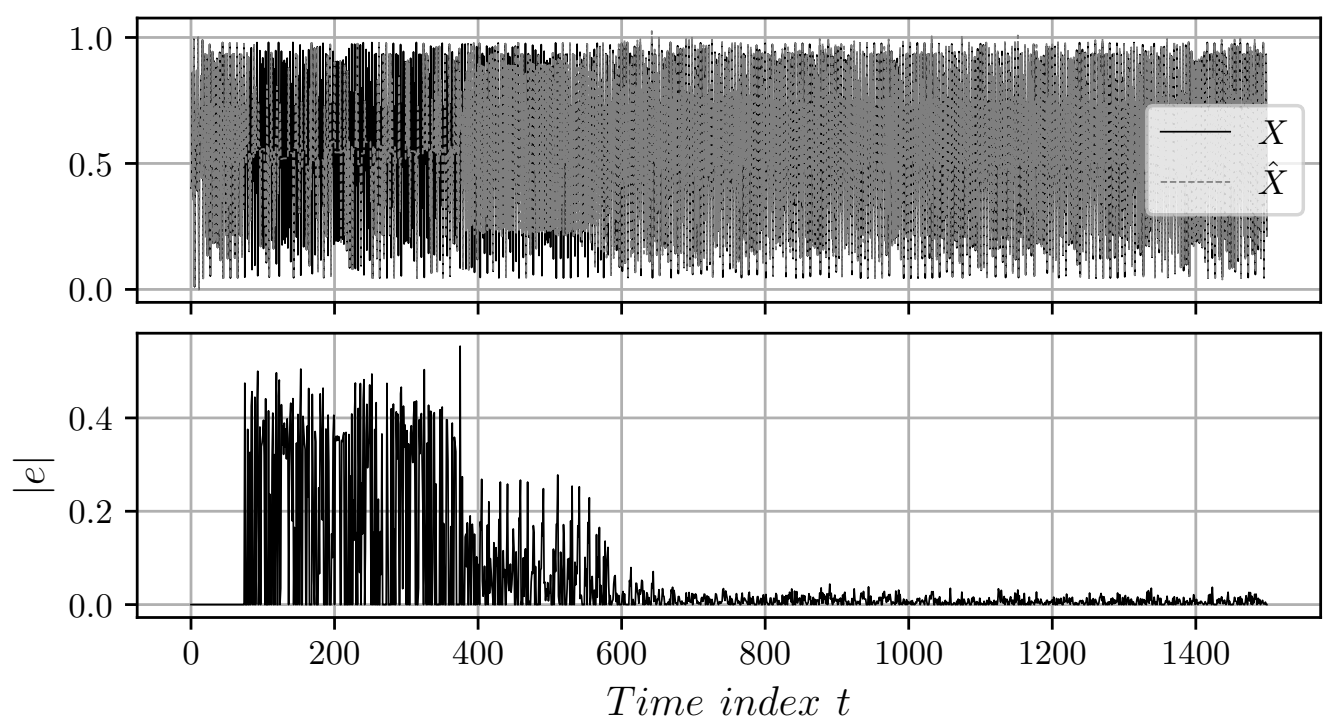

Figure B.54: Top: $X$ and $\hat{X}$. Bottom: Instantaneous absolute error $|e|$. 


\section{S11 with PSF=High Entropy}

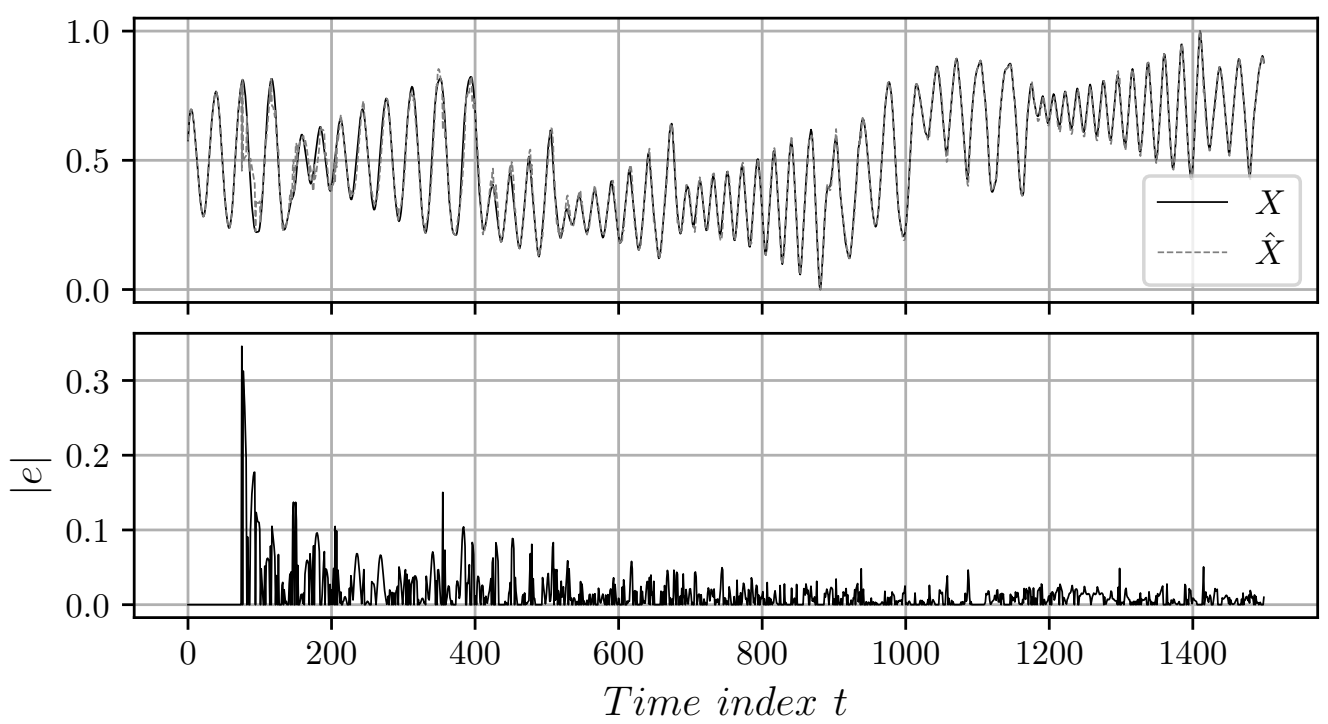

Figure B.55: Top: $X$ and $\hat{X}$. Bottom: Instantaneous absolute error $|e|$. 